\title{
Árvores entrelaçadoras de polinômios e grafos de Ramanujan
}

\author{
Karina Suemi Awoki
DISSERTAÇÃO APRESENTADA
$\mathrm{AO}$
Instituto DE MATEMÁticA E Estatística
DA
Universidade DE SÃo PAUlo
PARA
OBTENÇÃO DO TÍTULO
$\mathrm{DE}$
Mestre em CiÊNCIAS \\ Programa: Ciência da computação \\ Orientador: Prof. Dr. Marcel Kenji de Carli Silva
}

Durante o desenvolvimento deste trabalho a autora recebeu auxílio financeiro da CAPES

São Paulo, junho de 2020 


\section{Árvores entrelaçadoras de polinômios e grafos de Ramanujan}

Esta versão da dissertação contém as correções e alterações sugeridas pela Comissão Julgadora durante a defesa da versão original do trabalho, realizada em 19/06/2020. Uma cópia da versão original está disponível no

Instituto de Matemática e Estatística da Universidade de São Paulo.

Comissão Julgadora:

- Prof ${ }^{\mathrm{a}}$. Dr ${ }^{\mathrm{a}}$. Cristina Gomes Fernandes - IME-USP

- Prof. Dr. Aritanan Borges Garcia Gruber - UFABC

- Prof. Dr. Daniel Morgato Martin - UFABC 


\section{Resumo}

Este trabalho tem como objetivo o estudo de grafos expansores, em particular, o estudo de técnicas de construção de famílias infinitas de grafos de Ramanujan regulares e de bons esparsificadores espectrais de grafos completos, ambos considerados bons grafos expansores. Dentre essas técnicas, estão a utilização de árvores entrelaçadoras de polinômios e a construção de grafos com funções barreira que limitam o crescimento de seus autovalores. Também estudaremos uma prova recente da resolução do Problema de Kadison-Singer por Marcus, Spielman e Srivastava, que utiliza uma combinação das técnicas de construção de bons expansores citadas anteriormente. 


\section{Abstract}

The goal of this work is to study expander graphs, particularly techniques to construct infinite families of (regular) Ramanujan graphs and spectral sparsifiers of complete graphs, both considered good expander graphs. Among these techniques are the use of interlacing trees of polynomials and the construction of graphs using barrier functions to bound eigenvalue growth. We also study a recent proof of the resolution of the Kadison-Singer Problem due to Marcus, Spielman, and Srivastava, which uses a combination of the previously mentioned techniques for constructing good expanders. 


\section{Sumário}

1 Introdução $\quad 9$

2 Preliminares 12

2.1 Tabelas de notações . . . . . . . . . . . . . . . . . . . . . . 12

2.2 Especificação de indeterminadas . . . . . . . . . . . . . . . . . . . . 15

2.3 Álgebra linear . . . . . . . . . . . . . . . . . . . . . . . 20

2.4 A pseudo-inversa de Moore-Penrose . . . . . . . . . . . . . . . . . . . 25

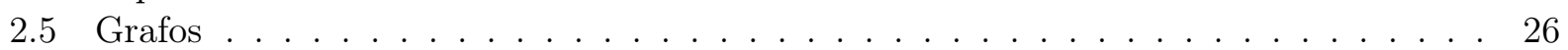

2.6 Teoria espectral dos grafos . . . . . . . . . . . . . . . . . . . . . 28

2.7 Polinômios de emparelhamentos . . . . . . . . . . . . . . . . . . . . 31

3 Grafos expansores $\quad 35$

3.1 Caracterização espectral de grafos expansores . . . . . . . . . . . . . . 36

3.2 Propriedades expansoras . . . . . . . . . . . . . . . . . . . 37

3.3 Grafos de Ramanujan . . . . . . . . . . . . . . . . . . . . . . . . 41

3.4 Expansores irregulares . . . . . . . . . . . . . . . . . . 46

4 Árvores entrelaçadoras de polinômios $\quad 47$

4.1 Entrelaçamento de polinômios . . . . . . . . . . . . . . . . . 47

4.2 Famílias entrelaçadoras de polinômios . . . . . . . . . . . . . . . 53

4.3 Provando que uma árvore de polinômios é entrelaçadora . . . . . . . . . . . . . 55

4.3.1 Ferramentas de análise . . . . . . . . . . . . . . . . . 55

4.3.2 Raízes de combinações convexas de polinômios . . . . . . . . . . . . . . 58

5 Polinômios estáveis $\quad 62$

5.1 Caracterizações de estabilidade . . . . . . . . . . . . . . . . . . 64

5.2 Preservadores de estabilidade . . . . . . . . . . . . . . . . 65

5.3 Exemplos de polinômios estáveis . . . . . . . . . . . . . . . . . . . 69

5.3 .1 Matrizes de sinais . . . . . . . . . . . . . . . 76

5.3.2 Mixed Characteristic Polynomial . . . . . . . . . . . . . . . . 77

5.4 Aplicações em árvores entrelaçadoras de polinômios . . . . . . . . . . . . . . . . 81

6 Famílias infinitas de grafos de Ramanujan $\quad 85$

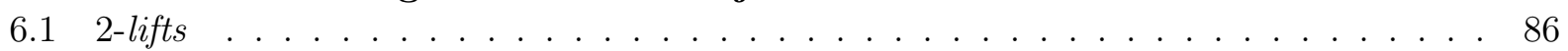

6.2 Polinômios de emparelhamentos . . . . . . . . . . . . . . . . . . . . . . 91

6.2.1 Raízes de polinômios de emparelhamentos . . . . . . . . . . . . . . . 94

6.3 Construção de grafos expansores . . . . . . . . . . . . . . . . . . . . . 99

6.4 Construção de grafos expansores irregulares . . . . . . . . . . . . . . . . 101 
7 Construção de expansores por esparsificadores espectrais $\quad 103$

7.1 Funções potenciais e barreiras . . . . . . . . . . . . . . . . . . . . . 104

7.2 Construção de expansores irregulares . . . . . . . . . . . . . . . . . 111

7.3 Propriedades expansoras de bons esparsificadores espectrais de grafos completos . . . 116

8 Problema de Kadison-Singer

120

8.1 Mixed Characteristic Polynomial . . . . . . . . . . . . . . . . . . . 121

8.2 Barreiras e função potencial . . . . . . . . . . . . . . . . . . . . . . . . 124

8.3 Resolução do problema de Kadison-Singer . . . . . . . . . . . . . . . . . . . 133

Bibliografia 


\section{Capítulo 1}

\section{Introdução}

Grafos expansores têm sido muito estudados devido às suas inúmeras aplicações, não só em ciência da computação, como também em matemática. Suas propriedades os tornam úteis na construção de redes de comunicação, na construção de códigos corretores de erros e na amplificação da probabilidade de sucesso em algoritmos probabilísticos (vide [17]).

Definiremos grafos expansores com mais rigor ao longo do texto, porém, por enquanto, o faremos informalmente para dar uma intuição ao leitor com a seguinte definição: grafos expansores são grafos esparsos e altamente conexos. Entenda altamente conexo por um grafo onde é necessário remover um número proporcionalmente grande de arestas para desconectar dois subconjuntos de vértices de tamanho grande. Isso não é o mesmo que dizer que o grafo é $\alpha$-aresta-conexo, pois, neste caso, é necessário remover pelo menos $\alpha$ arestas do grafo para torná-lo desconexo, não importando o tamanho dos subconjuntos de vértices a serem desconectados. Para mensurar a conectividade nas arestas de um grafo, utilizamos o índice de expansão, que é um valor definido de forma combinatória, de modo que quanto maior o índice de expansão de um grafo, melhores são as suas características expansoras. Assim, para obter bons expansores, utilizaremos construções de grafos esparsos com alto índice de expansão. Pode-se dizer que grafos completos possuem índice de expansão máximo e, portanto, são altamente conexos. Porém estes não devem ser chamados de expansores, pois não são grafos esparsos.

Devido à sua estrutura, grafos expansores possuem três propriedades principais, que são:

1. alta condutibilidade nas arestas, ou seja, é preciso remover um número proporcionalmente grande de arestas para desconectar dois subconjuntos de vértices que possuem um grande número de arestas incidentes,

2. o número de arestas entre dois subconjuntos de vértices quaisquer de um grafo expansor tem um comportamento semelhante ao de grafos aleatórios, e

3. passeios aleatórios em grafos expansores convergem rapidamente para a distribuição estacionária, que é uniforme caso o grafo seja regular.

Seja $G$ um grafo regular. Um resultado de Cheeger [7] e Alon e Milman [1] nos dá uma aproximação do índice de expansão de $G$ pelo seu gap espectral, que é a diferença entre os dois maiores autovalores da matriz de adjacência de $G$. Segundo esse resultado, o índice de expansão de $G$ é grande se e somente se seu gap espectral é grande. Dessa forma, se $G$ é um grafo esparso com um número grande de vértices e seu gap espectral é grande, então $G$ é um bom expansor. Para mostrar a existência de bons expansores, utilizaremos a caracterização espectral do índice de expansão no lugar da definição combinatória, pois a primeira nos permite utilizar resultados de álgebra linear, 
além de ser mais simples calcular uma aproximação espectral do índice de expansão do que seu valor exato de forma combinatória.

Alon e Boppana (vide [27]) mostraram um resultado que limita superiormente, de forma assintótica, o gap espectral de grafos $d$-regulares por $d-2 \sqrt{d-1}$. Por esse motivo, consideramos grafos esparsos $d$-regulares, com $d>2$, com um grande número de vértices e que atingem esse limitante como expansores ótimos. Como exemplos de expansores ótimos temos os grafos de Ramanujan, que são grafos conexos $d$-regulares, com $d>2$, onde os valores absolutos de seus autovalores não-triviais (autovalores cujos valores absolutos diferem de $d$ ) são no máximo $2 \sqrt{d-1}$. Dessa forma, estamos interessados em construir famílias infinitas de grafos de Ramanujan de mesma valência com um número crescente de vértices, o que garante que essas famílias contêm expansores ótimos com um número linear de arestas em relação ao número de vértices.

Em 1988, Lubotzky, Phillips e Sarnak [23] e Margulis [26] foram os primeiros a provar a existência de famílias infinitas de grafos de Ramanujan. Essas famílias são famílias de grafos de Cayley $(p+1)$ regulares, com $p$ primo. Depois disso, foi provada a existência de outras famílias de grafos de Ramanujan, mas todas eram famílias de grafos $(p+1)$-regulares, com $p$ uma potência de um primo. Portanto, só eram conhecidas famílias de grafos de Ramanujan de valências específicas, até que Marcus, Spielman e Srivastava [24] provaram, em 2013, a existência de famílias infinitas de grafos de Ramanujan bipartidos para todas as valências maiores que 2. Além disso, provaram também a existência de famílias infinitas de grafos de Ramanujan bipartidos não regulares com uma alteração na definição de grafos de Ramanujan.

Para provar a existência de expansores ótimos de qualquer valência $d$ maior que 2 , foi mostrado em [24] que é possível construir uma família infinita de grafos de Ramanujan $d$-regulares com um número crescente de vértices a partir de um grafo de Ramanujan bipartido $d$-regular. Primeiramente, a cada grafo de Ramanujan bipartido, associamos certo conjunto de grafos bipartidos com o dobro do número de vértices e com a mesma valência do grafo original. Mostraremos que pelo menos um dos grafos desse conjunto é um grafo de Ramanujan utilizando propriedades de polinômios estáveis e árvores entrelaçadoras de polinômios. Árvores entrelaçadoras de polinômios são árvores cujos nós representam polinômios que obedecem certa restrição, e possuem uma propriedade que limita superiormente as raízes de pelo menos um de seus polinômios folha pela raiz máxima da soma de todos os seus polinômios folha. Essa propriedade será utilizada para limitar superiormente, por $2 \sqrt{d-1}$, os autovalores da matriz de adjacência de pelo menos um grafo pertencente ao conjunto associado a um grafo de Ramanujan bipartido. Assim, é possível utilizar árvores entrelaçadoras de polinômios para mostrar a existência de uma função injetora que associa qualquer grafo de Ramanujan bipartido a um grafo de Ramanujan bipartido de mesma valência com o dobro do número de vértices do grafo original. Utilizamos essa função para construir famílias infinitas de grafos de Ramanujan de mesma valência iterativamente. Primeiramente, escolhemos um grafo de Ramanujan bipartido de valência $d$, como por exemplo o grafo bipartido completo $K_{d, d}$, e o adicionamos à família. Depois disso, basta utilizar a função mencionada anteriormente para encontrar o próximo grafo a ser inserido na família a partir do último grafo adicionado. Assim, repetindo o processo a cada grafo adicionado, é possível construir famílias infinitas de grafos de Ramanujan de qualquer valência com um número crescente de vértices.

Batson, Spielman e Srivastava [2] provaram a existência de grafos expansores quase ótimos utilizando outra definição de expansores que considera grafos não-regulares e que satisfaz as três propriedades expansoras citadas anteriormente. Esse resultado segue da prova construtiva de existência de bons esparsificadores espectrais de um grafo $G$, que são subgrafos esparsos de $G$ cujas formas quadráticas de suas matrizes Laplacianas são próximas às formas quadráticas do Laplaciano de $G$. Como grafos de Ramanujan não-bipartidos podem ser considerados esparsificadores espectrais muito bons de grafos completos, então faz sentido considerarmos bons esparsificadores espectrais 
como grafos que são aproximações pelo menos tão boas quanto a aproximação de grafos completos por grafos de Ramanujan não-bipartidos. A existência de bons esparsificadores espectrais de grafos arbitrários foi provada de forma construtiva em [2], utilizando técnicas de controle de autovalores por barreiras e funções potenciais. A ideia da prova é construir certa matriz com autovalores próximos de 1 e aplicar algumas operações nessa matriz com o objetivo de transformá-la no Laplaciano de um subgrafo que é um bom esparsificador espectral do grafo original. Essa matriz é obtida de uma prova construtiva, onde, a cada iteração, é realizada uma atualização de posto 1 na matriz em progresso, que causa um aumento controlado nos autovalores da matriz em construção. As matrizes referentes a cada iteração representam um grafo e cada atualização de posto 1 adiciona uma aresta com peso a esse grafo. Para controlar o aumento dos autovalores, utilizamos barreiras que limitam inferior e superiormente os autovalores das matrizes em progresso. Essas barreiras aumentam de forma constante, de modo que, depois de um grande número de iterações, todos os autovalores da matriz em progresso estão em um intervalo pequeno se comparado à distância entre os autovalores e o ponto zero. Utilizamos as funções potenciais, que aumentam à medida que os autovalores se aproximam das barreiras, para não permitir que os autovalores as ultrapassem. Consideramos a matriz final como a matriz em progresso após um grande número de iterações. Assim, ao dividir a matriz final pelo seu menor autovalor, obtemos uma matriz com autovalores próximos de 1, que representa um bom esparsificador espectral do grafo original.

Combinando as técnicas de construção de bons expansores apresentadas em [24] e [2], Marcus, Spielman e Srivastava [25] resolveram o Problema de Kadison-Singer, que estava em aberto desde 1959 até o momento. O Problema de Kadison-Singer é um problema de análise funcional que tem duas possíveis respostas: a positiva e a negativa. Na tentativa de resolver esse problema, foram criadas algumas conjecturas equivalentes às suas possíveis soluções, e uma delas foi provada por Marcus, Spielman e Srivastava. Para prová-la, eles mostraram que para todo $\varepsilon>0$ existe uma matriz, pertencente a certo conjunto finito de matrizes $M$, que possui autovalores limitados superiormente por $(1+\sqrt{\varepsilon})^{2}$. Para mostrar a existência dessa matriz, eles utilizam árvores entrelaçadoras de polinômios e a técnica de limitar os autovalores com barreiras, ambas citadas anteriormente. Defina $P$ como o conjunto dos polinômios característicos das matrizes em $M$. Primeiramente, as raízes da soma dos polinômios do conjunto finito $P$ são limitadas superiormente por $(1+\sqrt{\varepsilon})^{2}$ com o auxílio de barreiras. Depois, mostramos que os polinômios em $P$ compõem as folhas de uma árvore entrelaçadora de polinômios e utilizamos uma de suas propriedades para mostrar que as raízes de pelo menos um desses polinômios são limitadas superiormente pela raiz máxima da soma de todos os polinômios em $P$. Como os autovalores de uma matriz são as raízes do seu polinômio característico, então, ao limitar as raízes de pelo menos um polinômio em $P$ por um valor, estamos também limitando os autovalores de uma matriz em $M$ pelo mesmo valor. Dessa forma, foi provada uma das conjecturas do Problema de Kadison-Singer equivalente a resposta positiva, utilizando as mesmas técnicas usadas na construção de bons expansores.

Neste texto, também foram utilizados alguns resultados de teoria espectral de grafos, análise real para mostrar a continuidade de autovalores e teoria de polinômios para mostrar propriedades de polinômios estáveis e de polinômios que se entrelaçam. 


\section{Capítulo 2}

\section{Preliminares}

Neste capítulo, apresentaremos alguns resultados de álgebra linear, pseudo-inversas de matrizes, especificação de polinômios, grafos, teoria espectral dos grafos e polinômios de emparelhamentos, entre outros. Os resultados foram aqui apresentados para serem consultados de acordo com a preferência do leitor.

\subsection{Tabelas de notações}

Utilize as tabelas 2.1, 2.2 e 2.3 a seguir para eventuais consultas sobre notações. Considere $G$ como um grafo, $X$ como um conjunto e $V$ e $W$ como conjuntos finitos.

Tabela 2.1: Notação de grafos

\begin{tabular}{rlrl}
\hline$V(G)$ & $:=$ conjunto de vértices de $G$ & \\
$E(G)$ & $:=$ conjunto de arestas de $G$ & & \\
$A_{G}$ & $:=$ matriz de adjacência de $G$ & & \\
$\delta_{G}(U)$ & $:=\{e \in E(G):|e \cap U|=1\}$ & & para $U \subseteq V(G)$ \\
$\delta_{G}(v)$ & $:=\delta_{G}(\{v\})$ & & para $v \in V(G)$ \\
$\Gamma_{G}(v)$ & $:=\{u \in V(G):\{u, v\} \in E(G)\}$ & & para $v \in V(G)$ \\
$\operatorname{grau}_{G}(v)$ & $:=\left|\Gamma_{G}(v)\right|$ & & para $v \in V(G)$ \\
$\Delta(G)$ & $:=\max \left\{\operatorname{grau}_{G}(v): v \in V(G)\right\}$ & & \\
$\rho(G)$ & $:=\max \left\{\left|\lambda_{1}^{\downarrow}\left(A_{G}\right)\right|,\left|\lambda_{1}^{\uparrow}\left(A_{G}\right)\right|\right\}$ & & \\
$\lambda(G)$ & $:=\max \left\{\left|\lambda_{2}^{\downarrow}\left(A_{G}\right)\right|,\left|\lambda_{1}^{\uparrow}\left(A_{G}\right)\right|\right\}$ & \\
$E_{G}(S, T)$ & $:=\{(u, v) \in S \times T:\{u, v\} \in E(G)\}$ & & para $S, T \subseteq V(G)$ \\
\hline
\end{tabular}




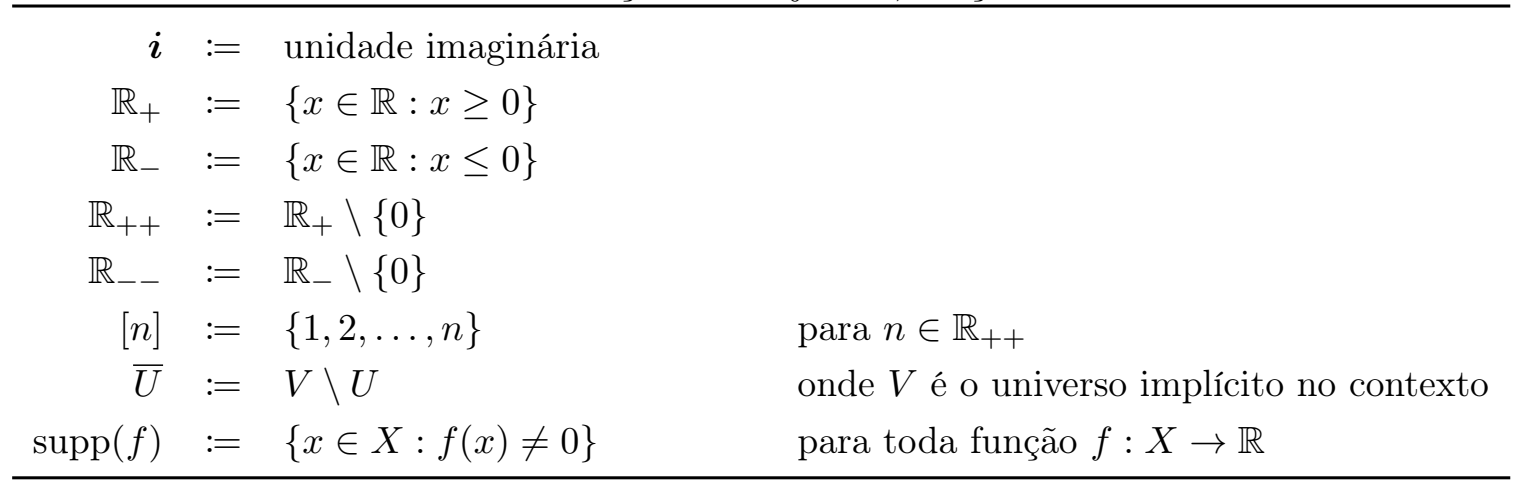

Tabela 2.3: Notação de vetores e matrizes

\begin{tabular}{|c|c|c|c|}
\hline$\oplus$ & $:=$ & soma direta de vetores & \\
\hline $\mathbb{1}$ & : & $U \rightarrow\{1\}$ & com $U$ implícito no contexto \\
\hline$J$ & : & $U \times U \rightarrow\{1\}$ & com $U$ implícito no contexto \\
\hline$I$ & $:=$ & matriz identidade & de dimensão implícita no contexto \\
\hline $\mathbb{1}_{S}$ & $:=$ & vetor característico de $S$ & de dimensão implícita no contexto \\
\hline$e_{u}$ & $:=$ & $\mathbb{1}_{\{u\}}$ & de dimensão implícita no contexto \\
\hline $\bar{A}$ & $:=$ & conjugado de $A$ & para $A \in \mathbb{C}^{W \times V}$ \\
\hline$A^{\top}$ & $:=$ & transposto de $A$ & para $A \in \mathbb{C}^{W \times V}$ \\
\hline$A^{*}$ & $:=$ & $(\bar{A})^{\top}$ & para $A \in \mathbb{C}^{W \times V}$ \\
\hline$P_{U}$ & $:=$ & projetor ortogonal de $U$ & para todo espaço vetorial $U$ \\
\hline$U^{\perp}$ & $:=$ & $\left\{x \in \mathbb{R}^{V}: y^{\top} x=0 \quad \forall y \in U\right\}$ & para $U \subseteq \mathbb{R}^{V}$ \\
\hline$\rho(A)$ & $:=$ & $\max \left\{\left|\lambda_{1}^{\downarrow}(A)\right|,\left|\lambda_{1}^{\uparrow}(A)\right|\right\}$ & para $A \in \mathbb{C}^{V \times V}$ \\
\hline$\|x\|_{2}$ & $:=$ & $\sqrt{x^{*} x}$ & para $x \in \mathbb{C}^{V}$ \\
\hline$\|A\|_{2}$ & $:=$ & $\max \left\{\|A x\|_{2}: x \in \mathbb{C}^{V}\right.$ e $\left.\|x\|_{2}=1\right\}$ & para $A \in \mathbb{C}^{V \times V}$ \\
\hline $\operatorname{adj}(A)$ & $:=$ & matriz adjunta de $A$ & para $A \in \mathbb{C}^{V \times V}$ \\
\hline $\operatorname{ker}(A)$ & $:=$ & $\left\{x \in \mathbb{R}^{V}: A x=0\right\}$ & para $A \in \mathbb{R}^{W \times V}$ \\
\hline $\operatorname{span}(U)$ & $:=$ & $\left\{\sum_{x \in X} a_{x} x: X \subseteq U\right.$ finito e $a \in \mathbb{R}^{X}$ & para $U \subseteq \mathbb{R}^{V}$ \\
\hline $\operatorname{Diag}(x)$ & $:=$ & a matriz diagonal com diagonal $x$ & para $x \in \mathbb{C}^{V}$ \\
\hline
\end{tabular}


Definição 2.1 (Conjunto conexo). Seja $\Omega \subseteq \mathbb{C}$. Dizemos que $\Omega$ é conexo se, para todos $S_{1}, S_{2} \subseteq \mathbb{C}$ disjuntos e abertos em $\Omega$, vale que $S_{1} \cup S_{2} \neq \Omega$.

Definição 2.2. Seja $p \in \mathbb{R}[x]$ um polinômio com raízes reais. Denote por $r_{1}(p), \ldots, r_{m}(p)$ as raízes de $p$ tais que $r_{1}(p) \leq \cdots \leq r_{m}(p)$. Denote também $r_{\max }(p):=r_{m}(p)$.

Definição 2.3. Seja $p \in \mathbb{C}\left[x_{1}, \ldots, x_{m}\right]$. Sejam $A \subseteq \mathbb{N}^{m}$ finito e $c: A \rightarrow \mathbb{C}$ tais que

$$
p=\sum_{a \in A} c_{a} \prod_{i \in[m]} x_{i}^{a_{i}}
$$

Defina

$$
\left[\prod_{i \in[m]} x_{i}^{a_{i}}\right]\left(p\left(x_{1}, \ldots, x_{m}\right)\right):=c_{a}
$$

para todo $a \in A$.

Proposição 2.4 (Série de Neumann [18]). Seja $A \in \mathbb{C}^{n \times n}$. Se $\rho(A)<1$, então $(I-A)^{-1}=\sum_{i \in \mathbb{N}} A^{i}$.

Proposição 2.5. Sejam $f: \mathbb{R} \rightarrow \mathbb{R}$ e $p \in \mathbb{R}$. Se $\lim _{n \rightarrow \infty} f\left(p_{n}\right)=f(p)$ para toda sequência de números reais $\left(p_{n}\right)_{n \in \mathbb{N}}$ tal que $\lim _{n \rightarrow \infty} p_{n}=p$ e que $p_{n} \neq p$ para todo $n \in \mathbb{N}$, então

$$
\lim _{x \rightarrow p} f(x)=f(p) .
$$

Demonstração. Suponha que $\lim _{x \rightarrow p} f(x) \neq f(p)$. Assim, existe $\varepsilon>0$ tal que, para todo $\delta>0$ existe $x_{\delta} \in \mathbb{R}$ com $0<\left|p-x_{\delta}\right|<\delta$ tal que

$$
\left|f(p)-f\left(x_{\delta}\right)\right| \geq \varepsilon
$$

Defina $\delta_{n}:=\frac{1}{n+1}$ e $p_{n}:=x_{\delta_{n}}$ para todo $n \in \mathbb{N}$. Note que $\lim _{n \rightarrow \infty} p_{n}=p$. Porém, existe $\varepsilon>0$ tal que $\left|f(p)-f\left(p_{n}\right)\right| \geq \varepsilon$ para todo $n \in \mathbb{N}$. Portanto existe uma sequência $\left(p_{n}\right)_{n \in \mathbb{N}} \operatorname{com} \lim _{n \rightarrow \infty} p_{n}=p$ tal que $\lim _{n \rightarrow \infty} f\left(p_{n}\right) \neq f(p)$.

Lema 2.6 (Propriedade de Helly). Sejam $I_{1}, I_{2}, \ldots, I_{n}$ intervalos fechados em $\mathbb{R}$. Então vale que $\bigcap_{j \in[n]} I_{j} \neq \varnothing$ se e somente se $I_{i} \cap I_{j} \neq \varnothing$ para todo $i, j \in[n]$.

Demonstração. Suponha que $\bigcap_{j \in[n]} I_{j} \neq \varnothing$. É fácil ver que $I_{i} \cap I_{j} \neq \varnothing$ para todo $i, j \in[n]$.

Suponha agora que $I_{i} \cap I_{j} \neq \varnothing$ para todo $i, j \in[n]$. Defina $\alpha_{j}:=\min I_{j}$ e $\beta_{j}:=\max I_{j}$ para todo $j \in[n]$. Defina

$$
\alpha:=\max _{j \in[n]} \alpha_{j} \quad \text { e } \quad \beta:=\min _{j \in[n]} \beta_{j} .
$$

Como $I_{i} \cap I_{j} \neq \varnothing$ para todo $i, j \in[n]$, então $\alpha \leq \beta$. Logo, vale que $[\alpha, \beta] \neq \varnothing$. Note que, para todo $j \in[n]$, vale que $\alpha_{j} \leq \alpha$ e $\beta_{j} \geq \beta$. Logo, vale que $[\alpha, \beta] \subseteq I_{j}$ para todo $j \in[n]$. Portanto, vale que

$$
\bigcap_{j \in[n]} I_{j}=[\alpha, \beta]
$$




\subsection{Especificação de indeterminadas}

Especificação de uma indeterminada é uma função que mapeia um polinômio em outro, substituindo uma de suas indeterminadas por um polinômio. Sejam $p \in \mathbb{R}[x]$ um polinômio e $\alpha \in \mathbb{R}$. Dizemos que $p(\alpha)$ é o polinômio resultante da especificação de $p(x)$ que transforma a indeterminada $x$ em $\alpha$. Nesse caso, conseguimos representar essa especificação de forma simples sem utilizar notações extras, mas ao longo do texto utilizaremos especificações de polinômios multivariados e especificações de derivadas de polinômios. Como é mais complicado representar esses casos, faremos uso de uma notação diferente para especificações.

Seguem a definição e alguns resultados de especificações de indeterminadas.

Definição 2.7 (Especificação de indeterminadas). Sejam $x_{1}, \ldots, x_{m}, y_{1}, \ldots, y_{r}$ indeterminadas algebricamente independentes e $n \in \mathbb{N} \backslash\{0\}$. Defina $X:=\left\{x_{1}, \ldots, x_{m}\right\}$ e $Y:=\left\{y_{1}, \ldots, y_{r}\right\}$. Seja $X^{\prime} \subseteq X$ e seja $f: X^{\prime} \rightarrow \mathbb{C}[X \cup Y]$. Defina $E_{f}: \mathbb{C}^{n \times n}[X] \rightarrow \mathbb{C}^{n \times n}[X \cup Y]$ como

$$
E_{f}\left(\sum_{a \in A} C_{a} \prod_{i \in[m]} x_{i}^{a_{i}}\right)=\sum_{a \in A} C_{a} \prod_{i \in[m]: x_{i} \notin X^{\prime}} x_{i}^{a_{i}} \prod_{i \in[m]: x_{i} \in X^{\prime}}\left(f\left(x_{i}\right)\right)^{a_{i}},
$$

para todo $A \subseteq \mathbb{N}^{m}$ finito e toda função $C: A \rightarrow \mathbb{C}^{n \times n}$.

Sejam $x_{1}, \ldots, x_{m}$ indeterminadas algebricamente independentes e $\alpha \in \mathbb{C}^{m}$ um vetor. Seja $q \in \mathbb{C}\left[x_{1} \ldots, x_{m}, y_{1}, \ldots, y_{r}\right]^{m}$. Para simplificar a notação, utilizaremos $p(\alpha)$ no lugar da especificação $\left(x_{1}, \ldots, x_{m}\right) \rightarrow \alpha$ no polinômio $p \in \mathbb{C}\left[x_{1}, \ldots, x_{m}\right]$. Porém, não utilizaremos essa notação quando o polinômio em questão envolve derivadas, pois ela não fica clara nesse caso. Além disso, utilizamos, em alguns casos, $E_{x=q}$ para representar a especificação $E_{f}$, onde $f:\left\{x_{1}, \ldots, x_{m}\right\} \mapsto$ $\mathbb{C}\left[x_{1} \ldots, x_{m}, y_{1}, \ldots, y_{r}\right]$ é uma função definida como $x_{i} \mapsto q_{i}$.

Proposição 2.8. Sejam $x_{1}, \ldots, x_{m}, y_{1}, \ldots, y_{r}$ indeterminadas algebricamente independentes e seja $n \in \mathbb{N} \backslash\{0\}$. Defina $X:=\left\{x_{1}, \ldots, x_{m}\right\}$ e $Y:=\left\{y_{1}, \ldots, y_{r}\right\}$. Sejam $X^{\prime} \subseteq X$ e $f: X^{\prime} \rightarrow \mathbb{C}[X \cup Y]$. Então, para todo $P_{1}, P_{2} \in \mathbb{C}^{n \times n}[X]$, vale que:

a) $E_{f}\left(P_{1}\right)+E_{f}\left(P_{2}\right)=E_{f}\left(P_{1}+P_{2}\right)$,

b) $E_{f}\left(P_{1}\right) E_{f}\left(P_{2}\right)=E_{f}\left(P_{1} P_{2}\right)$.

Demonstração. Sejam $A_{1}, A_{2} \subseteq \mathbb{N}^{m}$ finitos e sejam $C_{1}: A_{1} \rightarrow \mathbb{C}^{n \times n}$ e $C_{2}: A_{2} \rightarrow \mathbb{C}^{n \times n}$ tais que

$$
P_{1}=\sum_{a \in A_{1}} C_{1}(a) \prod_{i \in[m]} x_{i}^{a_{i}}, \quad P_{2}=\sum_{a \in A_{2}} C_{2}(a) \prod_{i \in[m]} x_{i}^{a_{i}}
$$

Note que

$$
\begin{aligned}
& E_{f}\left(\sum_{a \in A_{1}} C_{1}(a) \prod_{i \in[m]} x_{i}^{a_{i}}\right)=\sum_{a \in A_{1}} C_{1}(a) \prod_{i \in[m]: x_{i} \notin X^{\prime}} x_{i}^{a_{i}} \prod_{i \in[m]: x_{i} \in X^{\prime}}\left(f\left(x_{i}\right)\right)^{a_{i}}, \\
& E_{f}\left(\sum_{a \in A_{2}} C_{2}(a) \prod_{i \in[m]} x_{i}^{a_{i}}\right)=\sum_{a \in A_{2}} C_{2}(a) \prod_{i \in[m]: x_{i} \notin X^{\prime}} x_{i}^{a_{i}} \prod_{i \in[m]: x_{i} \in X^{\prime}}\left(f\left(x_{i}\right)\right)^{a_{i}} .
\end{aligned}
$$

Seja $C: A_{1} \cup A_{2} \rightarrow \mathbb{C}^{n \times n}$ definido como

$$
C(a):= \begin{cases}C_{1}(a)+C_{2}(a) & \text { se } a \in A_{1} \cap A_{2} \\ C_{1}(a) & \text { se } a \in A_{1} \backslash A_{2} \\ C_{2}(a) & \text { se } a \in A_{2} \backslash A_{1} .\end{cases}
$$


Dessa forma, vale que

$$
\begin{aligned}
E_{f}\left(P_{1}\right) & +E_{f}\left(P_{2}\right) \\
& =\sum_{a \in A_{1}}\left(C_{1}(a) \prod_{\substack{i \in[m]: \\
x_{i} \notin X^{\prime}}} x_{i}^{a_{i}} \prod_{\substack{i \in[m]: \\
x_{i} \in X^{\prime}}}\left(f\left(x_{i}\right)\right)^{a_{i}}\right)+\sum_{a \in A_{2}}\left(C_{2}(a) \prod_{\substack{i \in[m]: \\
x_{i} \notin X^{\prime}}} x_{i}^{a_{i}} \prod_{\substack{i \in[m]: \\
x_{i} \in X^{\prime}}}\left(f\left(x_{i}\right)\right)^{a_{i}}\right) \\
& =\sum_{a \in A_{1} \cup A_{2}} C_{a} \prod_{\substack{i \in[m]: x_{i} \notin X^{\prime} \\
x_{i}}} \prod_{\substack{a_{i} \\
i \in[m]: x_{i} \in X^{\prime}}}\left(f\left(x_{i}\right)\right)^{a_{i}} \\
& =E_{f}\left(\sum_{a \in A_{1} \cup A_{2}} C_{a} \prod_{i \in[m]: x_{i} \notin X^{\prime}} x_{i}^{a_{i}} \prod_{i \in[m]: x_{i} \in X^{\prime}} x_{i}^{a_{i}}\right) \\
& =E_{f}\left(\sum_{a \in A_{1}}\left(C_{1}(a) \prod_{\substack{i \in[m]: \\
x_{i} \notin X^{\prime}}} x_{i}^{a_{i}} \prod_{\substack{i \in[m]: \\
x_{i} \in X^{\prime}}} x_{i}^{a_{i}}\right)+\sum_{a \in A_{2}}\left(C_{2}(a) \prod_{\substack{i \in[m]: \\
x_{i} \notin X^{\prime}}} x_{i}^{a_{i}} \prod_{\substack{i \in[m]: \\
x_{i} \in X^{\prime}}} x_{i}^{a_{i}}\right)\right) \\
& =E_{f}\left(P_{1}+P_{2}\right) .
\end{aligned}
$$

Portanto, o item a) vale.

Além disso, também vale que

$$
\begin{aligned}
& E_{f}\left(P_{1}\right) E_{f}\left(P_{2}\right) \\
& =\left(\sum_{a \in A_{1}}\left(C_{1}(a) \prod_{\substack{i \in[m] ; \\
x_{i} \notin X^{\prime}}} x_{i}^{a_{i}} \prod_{\substack{i \in[m]: \\
x_{i} \in X^{\prime}}}\left(f\left(x_{i}\right)\right)^{a_{i}}\right)\right)\left(\sum_{a \in A_{2}}\left(C_{2}(a) \prod_{\substack{i \in[m]: \\
x_{i} \notin X^{\prime}}} x_{i}^{a_{i}} \prod_{\substack{i \in[m]: \\
x_{i} \in X^{\prime}}}\left(f\left(x_{i}\right)\right)^{a_{i}}\right)\right) \\
& =\sum_{\substack{a_{1} \in A_{1} \\
a_{2} \in A_{2}}} C_{1}\left(a_{1}\right) C_{2}\left(a_{2}\right) \prod_{i \in[m]: x_{i} \notin X^{\prime}} x_{i}^{a_{1}(i)+a_{2}(i)} \prod_{i \in[m]: x_{i} \in X^{\prime}}\left(f\left(x_{i}\right)\right)^{a_{1}(i)+a_{2}(i)} \\
& =E_{f}\left(\sum_{\substack{a_{1} \in A_{1} \\
a_{2} \in A_{2}}} C_{1}\left(a_{1}\right) C_{2}\left(a_{2}\right) \prod_{i \in[m]: x_{i} \notin X^{\prime}} x_{i}^{a_{1}(i)+a_{2}(i)} \prod_{i \in[m]: x_{i} \in X^{\prime}} x_{i}^{a_{1}(i)+a_{2}(i)}\right) \\
& =E_{f}\left(\left(\sum_{a \in A_{1}}\left(C_{1}(a) \prod_{\substack{i \in[m]: \\
x_{i} \notin X^{\prime}}} x_{i}^{a_{i}} \prod_{\substack{i \in[m]: \\
x_{i} \in X^{\prime}}} x_{i}^{a_{i}}\right)\right)\left(\sum_{a \in A_{2}}\left(C_{2}(a) \prod_{\substack{i \in[m]: \\
x_{i} \notin X^{\prime}}} x_{i}^{a_{i}} \prod_{\substack{i \in[m]: \\
x_{i} \in X^{\prime}}} x_{i}^{a_{i}}\right)\right)\right) \\
& =E_{f}\left(P_{1} P_{2}\right) .
\end{aligned}
$$

Portanto, o item b) vale.

Proposição 2.9. Sejam $x_{1}, \ldots, x_{m}, y_{1}, \ldots, y_{m}, z_{1}, \ldots, z_{m}$ indeterminadas algebricamente independentes. Denote $X:=\left\{x_{1}, \ldots, x_{m}\right\}, Y:=\left\{y_{1}, \ldots, y_{m}\right\}$ e $Z:=\left\{z_{1}, \ldots, z_{m}\right\}$. Sejam $f: Y \rightarrow \mathbb{C}[X]$ e $g: Z \rightarrow \mathbb{C}[X \cup Y]$. Defina $h: Z \rightarrow \mathbb{C}[X \cup Y]$ como $z \mapsto E_{f}(g(z))$. Então

$$
E_{f} \circ E_{g}=E_{h}
$$

Demonstração. Pela Definição 2.7, temos que $E_{f}: \mathbb{C}^{n \times n}[Y] \rightarrow \mathbb{C}^{n \times n}[X]$ e $E_{g}, E_{h}: \mathbb{C}^{n \times n}[Z] \rightarrow$ $\mathbb{C}^{n \times n}[X \cup Y]$. Seja $P \in \mathbb{C}^{n \times n}[Z]$ e sejam $A \subseteq \mathbb{N}^{m}$ finito e $C: A \rightarrow \mathbb{C}^{n \times n}$ tais que

$$
\sum_{a \in A} C_{a} \prod_{i \in[m]} z_{i}^{a_{i}}=P
$$


Note que

$$
\begin{aligned}
& E_{f}\left(E_{g}(P)\right)=E_{f}\left(E_{g}\left(\sum_{a \in A} C_{a} \prod_{i \in[m]} z_{i}^{a_{i}}\right)\right) \\
& =E_{f}\left(\sum_{a \in A} C_{a} \prod_{i \in[m]}\left(g\left(z_{i}\right)\right)^{a_{i}}\right) \\
& =\sum_{a \in A} C_{a} \prod_{i \in[m]}\left(E_{f}\left(g\left(z_{i}\right)\right)\right)^{a_{i}} \quad \text { pela Proposição } 2.8 \\
& =\sum_{a \in A} C_{a} \prod_{i \in[m]}\left(h\left(z_{i}\right)\right)^{a_{i}} \\
& =E_{h}\left(\sum_{a \in A} C_{a} \prod_{i \in[m]} z_{i}^{a_{i}}\right) .
\end{aligned}
$$

Proposição 2.10. Sejam $V$ e $W$ espaços vetoriais e seja $X: \Omega \rightarrow V$ uma variável aleatória de suporte finito. Seja $f: V \rightarrow W$ uma função linear. Então $f(\mathbb{E}(X))=\mathbb{E}(f(X))$.

Demonstração. Seja $P: \Omega \rightarrow \mathbb{R}_{+}$a distribuição de probabilidade associada a variável aleatória $X$. Note que

$$
f(\mathbb{E}(X))=f\left(\sum_{\omega \in \Omega} P(\omega) X(\omega)\right) .
$$

Assim, como $f$ é uma função linear, então

$$
f(\mathbb{E}(X))=\sum_{\omega \in \Omega} f(P(\omega) X(\omega))=\sum_{\omega \in \Omega} P(\omega) f(X(\omega))=\mathbb{E}(f(X)) .
$$

Proposição 2.11. Seja $X=\left\{x_{1}, \ldots, x_{m}\right\}$ um conjunto de indeterminadas algebricamente independentes. Sejam $X^{\prime} \subseteq X$ e $f: X^{\prime} \rightarrow \mathbb{C}$. Então

a) $E_{f}(\operatorname{det}(A))=\operatorname{det}\left(E_{f}(A)\right)$ para todo $A \in \mathbb{C}^{n \times n}[X]$,

b) $E_{f}(\operatorname{Tr}(A))=\operatorname{Tr}\left(E_{f}(A)\right)$ para todo $A \in \mathbb{C}^{n \times n}[X]$.

Demonstração. Seja $A \in \mathbb{C}^{n \times n}[X]$. Seja $S_{n}:=\{\pi:[n] \rightarrow[n]: \pi$ é bijetora $\}$ e seja $\operatorname{sgn}(\pi)$ o sinal da permutação $\pi$ para todo $\pi \in S_{n}$. Note que

$$
\begin{array}{rlr}
E_{f}(\operatorname{det}(A)) & =E_{f}\left(\sum_{\pi \in S_{n}} \operatorname{sgn}(\pi) \prod_{i \in[n]} A_{i, \pi(i)}\right) & \\
& =\sum_{\pi \in S_{n}} \operatorname{sgn}(\pi) \prod_{i \in[n]} E_{f}\left(A_{i, \pi(i)}\right) & \text { pela Proposição 2.8 } \\
& =\sum_{\pi \in S_{n}} \operatorname{sgn}(\pi) \prod_{i \in[n]}\left(E_{f}(A)\right)_{i, \pi(i)} & \\
& =\operatorname{det}\left(E_{f}(A)\right) .
\end{array}
$$

Logo, o item a) vale.

Note também que

$$
E_{f}(\operatorname{Tr}(A))=E_{f}\left(\sum_{i \in[n]} A_{i, i}\right)
$$




$$
\begin{array}{ll}
=\sum_{i \in[n]} E_{f}\left(A_{i, i}\right) & \text { pela Proposição } 2.8 \\
=\sum_{i \in[n]}\left(E_{f}(A)\right)_{i, i} &
\end{array}
$$

o que implica que o item b) vale.

Proposição 2.12. Sejam $X=\left\{x_{1}, \ldots, x_{m}\right\}$ um conjunto de indeterminadas algebricamente independentes e $i \in[m]$. Seja $X^{\prime} \subseteq X$ tal que $x_{i} \notin X^{\prime}$ e seja $f: X^{\prime} \rightarrow \mathbb{C}$. Então $\partial_{x_{i}} E_{f}(p)=E_{f}\left(\partial_{x_{i}} p\right)$ para todo $p \in \mathbb{C}[X]$.

Demonstração. Seja $p \in \mathbb{C}[X]$. Sejam $A \subseteq \mathbb{N}^{m}$ finito e $c: A \rightarrow \mathbb{C}$ tais que

$$
p=\sum_{a \in A} c_{a} \prod_{j \in[m]} x_{j}^{a_{j}} .
$$

Note que

$$
\begin{aligned}
\partial_{x_{i}} p & =\partial_{x_{i}}\left(\sum_{a \in A} c_{a} \prod_{j \in[m]} x_{j}^{a_{j}}\right) \\
& =\sum_{a \in A} c_{a} \partial_{x_{i}}\left(\prod_{j \in[m]} x_{j}^{a_{j}}\right) \\
& =\sum_{a \in A} c_{a} \partial_{x_{i}}\left(\prod_{j \in[m]: x_{j} \notin X^{\prime}} x_{j}^{a_{j}} \prod_{j \in[m]: x_{j} \in X^{\prime}} x_{j}^{a_{j}}\right) \\
& =\sum_{a \in A} c_{a}\left(\prod_{j \in[m]: x_{j} \in X^{\prime}} x_{j}^{a_{j}}\right) \partial_{x_{i}}\left(\prod_{j \in[m]: x_{j} \notin X^{\prime}} x_{j}^{a_{j}}\right) \\
& =\sum_{a \in A} c_{a}\left(\prod_{j \in[m]: x_{j} \in X^{\prime}} x_{j}^{a_{j}}\right)\left(\prod_{j \in[m]: x_{j} \notin\left(X^{\prime} \cup\left\{x_{i}\right\}\right)} x_{j}^{a_{j}}\right)\left(a_{i} x_{i}^{a_{i}-1}\right) .
\end{aligned}
$$

Logo, pela Proposição 2.8, temos que

$$
\begin{aligned}
E_{f}\left(\partial_{x_{i}} p\right) & =E_{f}\left(\sum_{a \in A} c_{a}\left(\prod_{\substack{j \in[m]: \\
x_{j} \in X^{\prime}}} x_{j}^{a_{j}}\right)\left(\prod_{\substack{j \in[m]: \\
x_{j} \notin\left(X^{\prime} \cup\left\{x_{i}\right\}\right)}} x_{j}^{a_{j}}\right)\left(a_{i} x_{i}^{a_{i}-1}\right)\right) \\
& =\sum_{a \in A} c_{a} E_{f}\left(\left(\prod_{\substack{j \in[m]: \\
x_{j} \in X^{\prime}}} x_{j}^{a_{j}}\right)\left(\prod_{\substack{j \in[m]: \\
x_{j} \notin\left(X^{\prime} \cup\left\{x_{i}\right\}\right)}} x_{j}^{a_{j}}\right)\left(a_{i} x_{i}^{a_{i}-1}\right)\right) \\
& =\sum_{a \in A} c_{a}\left(E_{f}\left(\prod_{\substack{j \in[m]: \\
x_{j} \in X^{\prime}}} x_{j}^{a_{j}}\right) E_{f}\left(\prod_{\substack{j \in[m]: \\
x_{j} \notin\left(X^{\prime} \cup\left\{x_{i}\right\}\right)}} x_{j}^{a_{j}}\right) E_{f}\left(a_{i} x_{i}^{a_{i}-1}\right)\right) \\
& =\sum_{a \in A} c_{a}\left(( \prod _ { \substack { j \in [ m ] : \\
x _ { j } \in X ^ { \prime } } } ( f ( x _ { j } ) ) ^ { a _ { j } } ) \left(\prod_{\substack{j \in[m]: \\
x_{j} \notin\left(X^{\prime} \cup\left\{x_{i}\right\}\right)}}^{\left.\left.x_{j}^{a_{j}}\right)\left(a_{i} x_{i}^{a_{i}-1}\right)\right) .}\right.\right.
\end{aligned}
$$

Pela Definição 2.7, temos que

$$
\partial_{x_{i}}\left(E_{f}(p)\right)=\partial_{x_{i}}\left(\sum_{a \in A} c_{a} \prod_{j \in[m]: x_{j} \notin X^{\prime}} x_{j}^{a_{j}} \prod_{j \in[m]: x_{j} \in X^{\prime}}\left(f\left(x_{j}\right)\right)^{a_{j}}\right) .
$$


Dessa forma, como $f(x) \in \mathbb{C}$ para todo $x \in X^{\prime}$, então

$$
\begin{aligned}
\partial_{x_{i}}\left(E_{f}(p)\right) & =\sum_{a \in A} c_{a}\left(\left(\prod_{\substack{j \in[m] ; \\
x_{j} \in X^{\prime}}}\left(f\left(x_{j}\right)\right)^{a_{j}}\right) \partial_{x_{i}}\left(\prod_{\substack{j \in[m]: \\
x_{j} \notin X^{\prime}}} x_{j}^{a_{j}}\right)\right) \\
& =\sum_{a \in A} c_{a}\left(\left(\prod_{\substack{j \in[m]: \\
x_{j} \in X^{\prime}}}\left(f\left(x_{j}\right)\right)^{a_{j}}\right)\left(\prod_{\substack{j \in[m]: \\
x_{j} \notin\left(X^{\prime} \cup\left\{x_{i}\right\}\right)}} x_{j}^{a_{j}}\right)\left(a_{i} x_{i}^{a_{i}-1}\right)\right) \\
& =E_{f}\left(\partial_{x_{i}} p\right)
\end{aligned}
$$

por (2.1).

Proposição 2.13. Seja $p \in \mathbb{R}\left(x_{1}, \ldots, x_{m}\right), i \in[m]$ e $a \in \mathbb{R}^{m}$. Então

$$
E_{x=a}\left(\partial_{x_{i}} p(x)\right)=\lim _{\delta \rightarrow 0} \frac{E_{x=a+\delta e_{i}}(p(x))-E_{x=a}(p(x))}{\delta} .
$$

Demonstração. Sejam $q, r \in \mathbb{R}\left[x_{1}, \ldots, x_{m}\right]$ tais que $p(x)=\frac{q(x)}{r(x)}$. Note que

$$
\partial_{x_{i}} p(x)=\frac{r(x) \partial_{x_{i}} q(x)-q(x) \partial_{x_{i}} r(x)}{r^{2}(x)} .
$$

Como $q$ e $r$ são polinômios, então ambos são deriváveis em $a_{i}$. Logo, vale que

$$
\begin{aligned}
E_{x=a}(r(x) & \left.\partial_{x_{i}} q(x)-q(x) \partial_{x_{i}} r(x)\right) \\
& =r(a) E_{x=a}\left(\partial_{x_{i}} q(x)\right)-q(a) E_{x=a}\left(\partial_{x_{i}} r(x)\right) \\
& =r(a)\left(\lim _{\delta \rightarrow 0} \frac{q\left(a+\delta e_{i}\right)-q(a)}{\delta}\right)-q(a)\left(\lim _{\delta \rightarrow 0} \frac{r\left(a+\delta e_{i}\right)-r(a)}{\delta}\right) \\
& =\lim _{\delta \rightarrow 0} \frac{r(a)\left(q\left(a+\delta e_{i}\right)-q(a)\right)}{\delta}-\lim _{\delta \rightarrow 0} \frac{q(a)\left(r\left(a+\delta e_{i}\right)-r(a)\right)}{\delta} \\
& =\lim _{\delta \rightarrow 0} \frac{r(a)\left(q\left(a+\delta e_{i}\right)-q(a)\right)-q(a)\left(r\left(a+\delta e_{i}\right)-r(a)\right)}{\delta} \\
& =\lim _{\delta \rightarrow 0} \frac{r(a) q\left(a+\delta e_{i}\right)-q(a) r\left(a+\delta e_{i}\right)}{\delta} .
\end{aligned}
$$

Assim, como $r(a)=\lim _{\delta \rightarrow 0} r(a+\delta)$, então

$$
\begin{aligned}
E_{x=a}\left(\partial_{x_{i}} p(x)\right) & =\frac{E_{x=a}\left(r(x) \partial_{x_{i}} q(x)-q(x) \partial_{x_{i}} r(x)\right)}{r^{2}(a)} \\
& =\frac{1}{r^{2}(a)} \lim _{\delta \rightarrow 0} \frac{r(a) q\left(a+\delta e_{i}\right)-q(a) r\left(a+\delta e_{i}\right)}{\delta} \\
& =\frac{1}{r(a) \lim _{\delta \rightarrow 0} r\left(a+\delta e_{i}\right)} \lim _{\delta \rightarrow 0} \frac{r(a) q\left(a+\delta e_{i}\right)-q(a) r\left(a+\delta e_{i}\right)}{\delta} \\
& =\lim _{\delta \rightarrow 0} \frac{r(a) q\left(a+\delta e_{i}\right)-q(a) r\left(a+\delta e_{i}\right)}{\delta r(a) r\left(a+\delta e_{i}\right)} \\
& =\lim _{\delta \rightarrow 0}\left(\frac{q\left(a+\delta e_{i}\right)}{\delta r\left(a+\delta e_{i}\right)}-\frac{q(a)}{\delta r(a)}\right) .
\end{aligned}
$$

Portanto, vale que $E_{x=a}\left(\partial_{x_{i}} p(x)\right)=\lim _{\delta \rightarrow 0} \frac{p\left(a+\delta e_{i}\right)-p(a)}{\delta}$. 


\section{3 Álgebra linear}

Nesta seção, mencionaremos alguns resultados de álgebra linear para eventuais consultas.

Definição 2.14. Seja $A \in \mathbb{C}^{V \times W}$ e sejam $S \subseteq V$ e $T \subseteq W$. Defina a matriz $A[S, T] \in \mathbb{C}^{S \times T}$ como $A[S, T](s, t):=A(s, t)$ para todos $s \in S$ e $t \in T$.

Proposição 2.15. Sejam $A \in \mathbb{C}^{m \times n}$ e $B \in \mathbb{C}^{n \times m}$. Então

$$
\operatorname{Tr}(A B)=\operatorname{Tr}(B A)
$$

Lema 2.16 (Desigualdade de Cauchy-Schwarz). Sejam $u, v \in \mathbb{R}^{n}$. Então,

$$
\left(u^{\top} v\right)^{2} \leq u^{\top} u v^{\top} v
$$

Demonstração. Sejam $i, j \in[n]$. Note que

$$
\left(u_{i} v_{j}\right)^{2}+\left(u_{j} v_{i}\right)^{2}-2 u_{i} v_{i} u_{j} v_{j}=\left(u_{i} v_{j}-u_{j} v_{i}\right)^{2} \geq 0 .
$$

Assim, vale que

$$
\begin{aligned}
\left(u^{\top} v\right)^{2} & =\left(\sum_{i \in[n]} u_{i} v_{i}\right)^{2}=\sum_{i \in[n]} u_{i}^{2} v_{i}^{2}+\sum_{i \in[n]} \sum_{j=i+1}^{n} 2 u_{i} v_{i} u_{j} v_{j} \\
& \leq \sum_{i \in[n]} u_{i}^{2} v_{i}^{2}+\sum_{i \in[n]} \sum_{j=i+1}^{n}\left(\left(u_{i} v_{j}\right)^{2}+\left(u_{j} v_{i}\right)^{2}\right) \\
& =\sum_{i \in[n]} u_{i}^{2} \sum_{i \in[n]} v_{i}^{2}=u^{\top} u v^{\top} v .
\end{aligned}
$$

Lema 2.17 (Fórmula de Sherman-Morrison [31]). Seja $A \in \mathbb{R}^{n \times n}$ uma matriz inversível e sejam $u, v \in \mathbb{R}^{n}$. Se $1+v^{\top} A^{-1} u \neq 0$, então

$$
\left(A+u v^{\top}\right)^{-1}=A^{-1}-\frac{A^{-1} u v^{\top} A^{-1}}{1+v^{\top} A^{-1} u} .
$$

Lema 2.18 (Matrix Determinant Lemma). Sejam $A \in \mathbb{C}^{n \times n}$ uma matriz inversível, $u, v \in \mathbb{C}^{n}$ e $x$ uma indeterminada. Então

$$
\operatorname{det}\left(A+x u v^{\top}\right)=\operatorname{det}(A)\left(1+x v^{\top} A^{-1} u\right) .
$$

Demonstração. Note que $A+x u v^{\top}=A\left(I+x A^{-1} u v^{\top}\right)$, o que implica que

$$
\operatorname{det}\left(A+x u v^{\top}\right)=\operatorname{det}(A) \operatorname{det}\left(I+x A^{-1} u v^{\top}\right) .
$$

Tome $w:=x A^{-1} u$. Note também que

$$
\left[\begin{array}{cc}
I & 0 \\
v^{\top} & 1
\end{array}\right]\left[\begin{array}{cc}
I+w v^{\top} & w \\
0 & 1
\end{array}\right]\left[\begin{array}{cc}
I & 0 \\
-v^{\top} & 1
\end{array}\right]=\left[\begin{array}{cc}
I+w v^{\top} & w \\
v^{\top}+v^{\top} w v^{\top} & v^{\top} w+1
\end{array}\right]\left[\begin{array}{cc}
I & 0 \\
-v^{\top} & 1
\end{array}\right]=\left[\begin{array}{cc}
I & w \\
0 & v^{\top} w+1
\end{array}\right] .
$$

Defina

$$
A_{1}:=\left[\begin{array}{cc}
I & 0 \\
v^{\top} & 1
\end{array}\right], \quad A_{2}:=\left[\begin{array}{cc}
I+w v^{\top} & w \\
0 & 1
\end{array}\right], \quad A_{3}:=\left[\begin{array}{cc}
I & 0 \\
-v^{\top} & 1
\end{array}\right] \quad \text { e } \quad B:=\left[\begin{array}{cc}
I & w \\
0 & v^{\top} w+1
\end{array}\right] .
$$


Assim, vale que $\operatorname{det}\left(A_{1}\right) \operatorname{det}\left(A_{2}\right) \operatorname{det}\left(A_{3}\right)=\operatorname{det}(B)$. Como $A_{1}$ e $A_{3}$ são matrizes triangulares com todas as entradas diagonais iguais a 1 , então $\operatorname{det}\left(A_{1}\right)=\operatorname{det}\left(A_{3}\right)=1$, o que implica que $\operatorname{det}\left(A_{2}\right)=$ $\operatorname{det}(B)$. Como $\operatorname{det}\left(A_{2}\right)=\operatorname{det}\left(I+w v^{\top}\right)$ e $\operatorname{det}(B)=v^{\top} w+1$, então

$$
\operatorname{det}\left(I+x A^{-1} u v^{\top}\right)=1+x v^{\top} A^{-1} u .
$$

Portanto, por (2.2), temos que

$$
\operatorname{det}\left(A+x u v^{\top}\right)=\operatorname{det}(A)\left(1+x v^{\top} A^{-1} u\right) .
$$

Definição 2.19. Seja $A \in \mathbb{C}^{n \times n}[x]$. Defina a matriz $\partial_{x} A \in \mathbb{C}^{n \times n}[x] \operatorname{como}\left(\partial_{x} A\right)(i, j):=\partial_{x} A(i, j)$ para todos $i, j \in[n]$.

Lema 2.20 (Fórmula de Jacobi). Seja $A \in \mathbb{C}^{n \times n}[x]$. Então

$$
\partial_{x} \operatorname{det}(A)=\operatorname{Tr}\left(\operatorname{adj}(A) \partial_{x} A\right) .
$$

Lema 2.21. Seja $x$ uma indeterminada e seja $\alpha \in \mathbb{R}$. Sejam $A, B \in \mathbb{C}^{n \times n}$ tais que $A+\alpha B$ é inversível. Então

$$
E_{x=\alpha}\left(\partial_{x} \operatorname{det}(A+x B)\right)=\operatorname{det}(A+\alpha B) \operatorname{Tr}\left((A+\alpha B)^{-1} B\right) .
$$

Demonstração. Pelo Lema 2.20, temos que

$$
\begin{array}{rlr}
E_{x=\alpha}\left(\partial_{x} \operatorname{det}(A+x B)\right) & =E_{x=\alpha}\left(\operatorname{Tr}\left(\operatorname{adj}(A+x B) \partial_{x}(A+x B)\right)\right) & \\
& =E_{x=\alpha}(\operatorname{Tr}(\operatorname{adj}(A+x B) B)) & \\
& =\operatorname{Tr}\left(E_{x=\alpha}(\operatorname{adj}(A+x B) B)\right) & \text { pela Proposição } 2.11 \\
& =\operatorname{Tr}\left(E_{x=\alpha}(\operatorname{adj}(A+x B)) E_{x=\alpha}(B)\right) & \text { pela Proposição 2.8 } \\
& =\operatorname{Tr}(\operatorname{adj}(A+\alpha B) B) .
\end{array}
$$

Como $\operatorname{det}(A+\alpha B) I=\operatorname{adj}(A+\alpha B)(A+\alpha B)$ e $A+\alpha B$ é inversível, então

$$
\operatorname{adj}(A+\alpha B)=\operatorname{det}(A+\alpha B)(A+\alpha B)^{-1} .
$$

Assim, vale que

$$
\begin{aligned}
E_{x=\alpha}\left(\partial_{x} \operatorname{det}(A+x B)\right) & =\operatorname{Tr}\left(\operatorname{det}(A+\alpha B)(A+\alpha B)^{-1} B\right) \\
& =\operatorname{det}(A+\alpha B) \operatorname{Tr}\left((A+\alpha B)^{-1} B\right) .
\end{aligned}
$$

Proposição 2.22. Sejam $p \in \mathbb{C}\left[x_{1}, \ldots, x_{m}\right]$ um polinômio e $i \in[m]$. Sejam $y, z$ indeterminadas algebricamente independentes. Então

$$
E_{x_{i}=y+z}\left(\partial_{x_{i}} p\left(x_{1}, \ldots, x_{m}\right)\right)=\partial_{y}\left(E_{x_{i}=y+z}\left(p\left(x_{1}, \ldots, x_{m}\right)\right)\right) .
$$

Demonstração. Sejam $A \subseteq \mathbb{N}^{m}$ finito e $c: A \rightarrow \mathbb{C}$ tais que $p=\sum_{a \in A} c_{a} \prod_{j \in[m]} x_{j}^{a_{j}}$. Note que

$$
E_{x_{i}=y+z}\left(\partial_{x_{i}} p\left(x_{1}, \ldots, x_{m}\right)\right)=E_{x_{i}=y+z}\left(\sum_{a \in A} c_{a} a_{i} x_{i}^{a_{i}-1} \prod_{j \in[m] \backslash\{i\}} x_{j}^{a_{j}}\right)
$$




$$
=\sum_{a \in A} c_{a} a_{i}(y+z)^{a_{i}-1} \prod_{j \in[m] \backslash\{i\}} x_{j}^{a_{j}} .
$$

Note também que

$$
\begin{aligned}
\partial_{y}\left(E_{x_{i}=y+z}\left(p\left(x_{1}, \ldots, x_{m}\right)\right)\right) & =\partial_{y}\left(\sum_{a \in A} c_{a}(y+z)^{a_{i}} \prod_{j \in[m] \backslash\{i\}} x_{j}^{a_{j}}\right) \\
& =\sum_{a \in A} c_{a} a_{i}(y+z)^{a_{i}-1} \prod_{j \in[m] \backslash\{i\}} x_{j}^{a_{j}} .
\end{aligned}
$$

Portanto, o resultado vale por (2.3) e (2.4).

Definição 2.23 (Matriz hermitiana). Seja $A \in \mathbb{C}^{V \times V}$. Dizemos que $A$ é hermitiana se $A=A^{*}$.

Proposição 2.24 (Matrizes hermitianas têm somente autovalores reais). Seja $A \in \mathbb{C}^{n \times n}$ uma matriz hermitiana. Então todos os autovalores de $A$ são reais.

Definição 2.25 (Autovalores). Seja $A \in \mathbb{C}^{n \times n}$ uma matriz hermitiana. Defina $\lambda^{\downarrow}(A) \in \mathbb{R}^{n}$ como o vetor de autovalores de $A$ tal que

$$
\lambda_{1}^{\downarrow}(A) \geq \cdots \geq \lambda_{n}^{\downarrow}(A)
$$

e $\operatorname{det}(x I-A)=\prod_{i \in[n]}\left(x-\lambda_{i}^{\downarrow}(A)\right)$ para todo $x \in \mathbb{C}$. Defina também $\lambda^{\uparrow}(A) \in \mathbb{R}^{n}$ tal que $\lambda_{i}^{\uparrow}(A)=$ $\lambda_{n+1-i}^{\downarrow}(A)$ para todo $i \in[n]$. Além disso, defina $\lambda_{\min }(A):=\lambda_{1}^{\uparrow}(A)$ e $\lambda_{\max }(A):=\lambda_{1}^{\downarrow}(A)$.

Definição 2.26 (Espectro). Seja $A \in \mathbb{C}^{n \times n}$ uma matriz hermitiana. Defina o espectro de $A$ como

$$
\operatorname{Spec}(A):=\left\{\lambda_{i}^{\downarrow}(A): i \in[n]\right\} .
$$

Definição 2.27 (Polinômio característico). Seja $A \in \mathbb{C}^{n \times n}$. Defina o polinômio $p_{A} \in \mathbb{C}[x]$ como $p_{A}(x):=\operatorname{det}(x I-A)$. Chamaremos $p_{A}$ de polinômio característico de $A$.

Proposição 2.28. Seja $A \in \mathbb{C}^{n \times n}$ e sejam $\lambda_{1}, \ldots, \lambda_{n}$ os autovalores de $A$. Então $\operatorname{det}(A)=\prod_{i \in[n]} \lambda_{i}$.

Demonstração. Seja $x$ uma indeterminada. Note que $p_{A}=\operatorname{det}(x I-A)=\prod_{i \in[n]}\left(x-\lambda_{i}\right) . \operatorname{Assim}$, tomando $x=0$, vale que

$$
\operatorname{det}(-A)=\prod_{i \in[n]}\left(-\lambda_{i}\right)
$$

o que implica que $(-1)^{n} \operatorname{det}(A)=(-1)^{n} \prod_{i \in[n]} \lambda_{i}$ e, portanto, $\operatorname{det}(A)=\prod_{i \in[n]} \lambda_{i}$.

Proposição 2.29. Seja $A \in \mathbb{C}^{n \times n}$ e sejam $\lambda_{1}, \ldots, \lambda_{n}$ os autovalores de $A$. Então $\operatorname{Tr}(A)=\sum_{i \in[n]} \lambda_{i}$.

Demonstração. Seja $x$ uma indeterminada. Note que existe um polinômio $q \in \mathbb{C}[x]$ de grau $d$ com $d \leq n-2$ tal que $\operatorname{det}(x I-A)=\prod_{i \in[n]}\left(x-A_{i, i}\right)+q$. Assim, vale que $\left[x^{n-1}\right](\operatorname{det}(x I-A))=-\operatorname{Tr}(A)$. Além disso, vale que

$$
\left[x^{n-1}\right]\left(\prod_{i \in[n]}\left(x-\lambda_{i}\right)\right)=-\sum_{i \in[n]} \lambda_{i} .
$$

Portanto, como $\operatorname{det}(x I-A)=\prod_{i \in[n]}\left(x-\lambda_{i}\right)$, então $\operatorname{Tr}(A)=\sum_{i \in[n]} \lambda_{i}$. 
Lema 2.30. Seja $A \in \mathbb{R}^{n \times n}$ uma matriz simétrica. Sejam $u_{1}, u_{2}, \ldots, u_{n} \in \mathbb{R}^{n}$ autovetores ortonormais de $A$ associados a $\lambda_{1}^{\downarrow}(A), \lambda_{2}^{\downarrow}(A), \ldots, \lambda_{n}^{\downarrow}(A)$, respectivamente. Tome $S_{i}:=\operatorname{span}\left(\left\{u_{1}, u_{2}, \ldots, u_{i}\right\}\right)$ para todo $i \in[n]$. Então

$$
\lambda_{i}^{\downarrow}(A)=\max \left\{\frac{x^{\top} A x}{x^{\top} x}: x \in S_{i-1}^{\perp} \text { e } x \neq 0\right\}
$$

para todo $i \in[n]$.

Demonstração. Seja $i \in[n]$ e seja $x \in S_{i-1}^{\perp} \operatorname{com} x \neq 0$. Como $\left\{u_{1}, \ldots, u_{n}\right\}$ é uma base de $\mathbb{R}^{n}$, então existem $\alpha_{i}, \ldots \alpha_{n} \in \mathbb{R}$ tais que $x=\sum_{j=i}^{n} \alpha_{j} u_{j}$. Assim, vale que

$$
\begin{aligned}
\frac{x^{\top} A x}{x^{\top} x} & =\frac{\left(\sum_{j=i}^{n} \alpha_{j} u_{j}^{\top}\right) A\left(\sum_{j=i}^{n} \alpha_{j} u_{j}\right)}{\left(\sum_{j=i}^{n} \alpha_{j} u_{j}^{\top}\right)\left(\sum_{j=i}^{n} \alpha_{j} u_{j}\right)} \\
& =\frac{\left(\sum_{j=i}^{n} \alpha_{j} u_{j}^{\top}\right) A\left(\sum_{j=i}^{n} \alpha_{j} u_{j}\right)}{\sum_{j=i}^{n} \alpha_{j}^{2}} \\
& =\frac{\left(\sum_{j=i}^{n} \alpha_{j} u_{j}^{\top}\right)\left(\sum_{j=i}^{n} \alpha_{j} \lambda_{j}^{\downarrow}(A) u_{j}\right)}{\sum_{j=i}^{n} \alpha_{j}^{2}} \\
& =\frac{\sum_{j=i}^{n} \alpha_{j}^{2} \lambda_{j}^{\downarrow}(A)}{\sum_{j=i}^{n} \alpha_{j}^{2}} \\
& \leq \frac{\lambda_{i}^{\downarrow}(A) \sum_{j=i}^{n} \alpha_{j}^{2}}{\sum_{j=i}^{n} \alpha_{j}^{2}}=\lambda_{i}^{\downarrow}(A) .
\end{aligned}
$$

Logo, vale que

$$
\lambda_{i}^{\downarrow}(A) \geq \max \left\{\frac{x^{\top} A x}{x^{\top} x}: x \in S_{i-1}^{\perp} \text { e } x \neq 0\right\} .
$$

Além disso, como $u_{i} \in S_{i-1}^{\perp}$ e $u_{i}^{\top} A u_{i}=\lambda_{i}^{\downarrow}(A)$, então

$$
\lambda_{i}^{\downarrow}(A) \leq \max \left\{\frac{x^{\top} A x}{x^{\top} x}: x \in S_{i-1}^{\perp} \text { e } x \neq 0\right\} .
$$

Definição 2.31 (Matrizes positivas semidefinidas). Sejam $A, B \in \mathbb{C}^{n \times n}$ matrizes hermitianas. Dizemos que $A \succeq B$ (ou $B \preceq A$ ) se $x^{*} A x \geq x^{*} B x$ para todo $x \in \mathbb{C}^{n}$. Dizemos também que $A$ é positiva semidefinida se $A \succeq 0$.

Proposição 2.32 (Autovalores de matrizes positivas semidefinidas). Seja $A \in \mathbb{C}^{n \times n}$ uma matriz positiva semidefinida. Então $\lambda_{i}^{\downarrow}(A) \geq 0$ para todo $i \in[n]$.

Demonstração. Seja $i \in[n]$ e seja $u_{i}$ um autovetor de $A$ associado a $\lambda_{i}^{\downarrow}(A)$. Note que $A u_{i}=\lambda_{i}^{\downarrow}(A) u_{i}$. Assim, vale que

$$
u_{i}^{*} A u_{i}=u_{i}^{*} \lambda_{i}^{\downarrow}(A) u_{i}=\lambda_{i}^{\downarrow}(A)\left\|u_{i}\right\|^{2} .
$$

Como $A$ é positiva semidefinida, então $u_{i}^{*} A u_{i} \geq 0$ e, portanto, vale que $\lambda_{i}^{\downarrow}(A) \geq 0$.

Definição 2.33 (Matrizes positivas definidas). Sejam $A, B \in \mathbb{C}^{n \times n}$ matrizes hermitianas. Dizemos que $A \succ B$ (ou $B \prec A$ ) se $x^{*} A x>x^{*} B x$ para todo $x \in \mathbb{C}^{n} \backslash\{0\}$. Dizemos também que $A$ é positiva definida se $A \succ 0$. 
Proposição 2.34 (Autovalores de matrizes positivas definidas). Seja $A \in \mathbb{C}^{n \times n}$ uma matriz positiva definida. Então $\lambda_{i}^{\downarrow}(A)>0$ para todo $i \in[n]$.

Demonstração. Sejam $i \in[n]$ e $u_{i}$ um autovetor de $A$ associado a $\lambda_{i}^{\downarrow}(A)$. Note que $A u_{i}=\lambda_{i}^{\downarrow}(A) u_{i}$. Dessa forma, vale que

$$
u_{i}^{*} A u_{i}=u_{i}^{*} \lambda_{i}^{\downarrow}(A) u_{i}=\lambda_{i}^{\downarrow}(A)\left\|u_{i}\right\|^{2} .
$$

Como $A$ é positiva definida, então $u_{i}^{*} A u_{i}>0$. Portanto, vale que $\lambda_{i}^{\downarrow}(A)>0$.

Proposição 2.35. Seja $A \in \mathbb{R}^{n \times n}$ uma matriz simétrica. Então $A \succeq 0$ se e somente se $x^{\top} A x \geq 0$ para todo $x \in \mathbb{R}^{n}$.

Demonstração. Pela Definição 2.31, é fácil ver que $A \succeq 0$ implica que $x^{\top} A x \geq 0$ para todo $x \in \mathbb{R}^{n}$.

Suponha agora que $x^{\top} A x \geq 0$ para todo $x \in \mathbb{R}^{n}$. Seja $y \in \mathbb{C}^{n}$. Note que existem $a, b \in \mathbb{R}^{n}$ tais que $a+\boldsymbol{i} b=y$. Note também que

$$
\begin{aligned}
y^{*} A y & =(a+\boldsymbol{i} b)^{*} A(a+\boldsymbol{i} b)=(a-\boldsymbol{i} b)^{\top} A(a+\boldsymbol{i} b) \\
& =a^{\top} A a+a^{\top} A \boldsymbol{i} b-\boldsymbol{i} b^{\top} A a-\boldsymbol{i} b^{\top} A \boldsymbol{i} b \\
& =a^{\top} A a+\boldsymbol{i} a^{\top} A b-\boldsymbol{i}\left(b^{\top} A a\right)^{\top}-\boldsymbol{i}^{2} b^{\top} A b \\
& =a^{\top} A a+\boldsymbol{i} a^{\top} A b-\boldsymbol{i} a^{\top} A^{\top} b+b^{\top} A b \\
& =a^{\top} A a+b^{\top} A b .
\end{aligned}
$$

Como $a, b \in \mathbb{R}^{n}$, então $a^{\top} A a \geq 0$ e $b^{\top} A b \geq 0$ e, portanto, vale que $a^{\top} A a+b^{\top} A b \geq 0$.

Proposição 2.36. Sejam $A, B \in \mathbb{C}^{n \times n}$ matrizes hermitianas tais que $A \succeq B$. Seja $C \in \mathbb{C}^{n \times m}$. Então $C^{*} A C \succeq C^{*} B C$.

Demonstração. Seja $x \in \mathbb{C}^{m}$. Defina $y:=C x$. Note que

$$
x^{*} C^{*} A C x=y^{*} A y \quad \text { e } \quad x^{*} C^{*} B C x=y^{*} B y .
$$

Como $A \succeq B$, então $y^{*} A y \geq y^{*} B y$, o que implica que $x^{*} C^{*} A C x \geq x^{*} C^{*} B C x$. Portanto, vale que $C^{*} A C \succeq C^{*} B C$.

Proposição 2.37. Seja $A \in \mathbb{C}^{n \times n}$ uma matriz positiva semidefinida. Então,

$$
\|A\|_{2}=\lambda_{1}^{\downarrow}(A) .
$$

Demonstração. Por definição, temos que

$$
\begin{aligned}
\|A\|_{2} & =\max \left\{\|A x\|_{2}: x \in \mathbb{C}^{n} \mathrm{e}\|x\|_{2}=1\right\} \\
& =\max \left\{\sqrt{x^{*} A^{2} x}: x \in \mathbb{C}^{n} \text { e }\|x\|_{2}=1\right\} \\
& =\sqrt{\lambda_{1}^{\downarrow}\left(A^{2}\right)}=\sqrt{\lambda_{1}^{\downarrow}(A)^{2}}
\end{aligned}
$$

pelo Lema 2.30.

Assim, pela Proposição 2.32, temos que $\lambda_{1}^{\downarrow}(A) \geq 0$ e, portanto, $\|A\|_{2}=\lambda_{1}^{\downarrow}(A)$. 


\subsection{A pseudo-inversa de Moore-Penrose}

Seguem alguns resultados referentes a matrizes pseudo-inversas.

Definição 2.38. Seja $A \in \mathbb{C}^{n \times n}$ e $\lambda \in \mathbb{C}$ um autovalor de $A$. Defina o autoespaço de $\lambda$ como

$$
E_{\lambda}:=\left\{v \in \mathbb{C}^{n}: A v=\lambda v\right\}
$$

Teorema 2.39 (Decomposição espectral [14]). Seja $A \in \mathbb{C}^{n \times n}$ uma matriz hermitiana. Então, vale que

$$
A=\sum_{\lambda \in \operatorname{Spec}(A)} \lambda P_{E_{\lambda}}
$$

Proposição 2.40. Seja $A \in \mathbb{R}^{n \times n}$ uma matriz simétrica de posto $m$. Então

$$
\left|\left\{i \in[n]: \lambda_{i}^{\downarrow}(A)=0\right\}\right|=n-m .
$$

Demonstração. Sejam $u_{1}, u_{2}, \ldots, u_{n}$ autovetores ortonormais associados a $\lambda_{1}^{\downarrow}(A), \lambda_{2}^{\downarrow}(A), \ldots, \lambda_{n}^{\downarrow}(A)$, respectivamente. Note que a dimensão de $\operatorname{ker}(A)$ é $n-m$. Note também que todo $v \in \operatorname{ker}(A)$ é um autovetor de $A$ associado ao autovalor 0 . Assim, como $u_{1}, \ldots, u_{n}$ são ortonormais, então

$$
\left|\left\{i \in[n]: u_{i} \in \operatorname{ker}(A)\right\}\right|=n-m \text {. }
$$

Definição 2.41 (Pseudo-inversa). Seja $A \in \mathbb{C}^{n \times n}$ uma matriz simétrica. Defina $S:=\operatorname{Spec}(A) \backslash\{0\}$. A pseudo-inversa de $A$ é a matriz

$$
A^{+}:=\sum_{\lambda \in S} \frac{1}{\lambda} P_{E_{\lambda}}
$$

Definição 2.42 (Raiz quadrada de matrizes). Seja $A \in \mathbb{C}^{n \times n}$ uma matriz positiva semidefinida. A raiz da matriz $A$ é a matriz

$$
A^{\frac{1}{2}}:=\sum_{\lambda \in \operatorname{Spec}(A)} \sqrt{\lambda} P_{E_{\lambda}} .
$$

Proposição 2.43. Sejam $A, B \in \mathbb{C}^{n \times n}$ matrizes positivas semidefinidas. Então $\operatorname{Tr}(A B) \geq 0$.

Demonstração. Note que $A^{\frac{1}{2}} A^{\frac{1}{2}}=A$. Assim, vale que

$$
\operatorname{Tr}(A B)=\operatorname{Tr}\left(A^{\frac{1}{2}} A^{\frac{1}{2}} B\right) .
$$

Logo, pela Proposição 2.15, temos que

$$
\operatorname{Tr}(A B)=\operatorname{Tr}\left(A^{\frac{1}{2}} B A^{\frac{1}{2}}\right)=\operatorname{Tr}\left(\left(A^{\frac{1}{2}}\right)^{*} B A^{\frac{1}{2}}\right)=\sum_{i \in[n]} e_{i}\left(A^{\frac{1}{2}}\right)^{*} B A^{\frac{1}{2}} e_{i}^{*} \geq 0,
$$

onde a desigualdade vale pela Proposição 2.36.

Proposição 2.44. Seja $A \in \mathbb{R}^{n \times n}$ uma matriz simétrica. Então

$$
A A^{+}=A^{+} A=P_{\operatorname{Im}(A)} .
$$


Demonstração. Sejam $u_{1}, u_{2}, \ldots, u_{n}$ autovetores ortonormais associados a $\lambda_{1}^{\downarrow}(A), \lambda_{2}^{\downarrow}(A), \ldots, \lambda_{n}^{\downarrow}(A)$, respectivamente.

Seja $S:=\operatorname{supp}\left(\lambda^{\downarrow}(A)\right)$. Pelo Teorema 2.39, temos que

$$
\begin{aligned}
A A^{+} & =\left(\sum_{i \in S} \lambda_{i}^{\downarrow}(A) u_{i} u_{i}^{\top}\right)\left(\sum_{i \in S} \frac{1}{\lambda_{i}^{\downarrow}(A)} u_{i} u_{i}^{\top}\right) \\
& =\left(\sum_{i \in S} \frac{1}{\lambda_{i}^{\downarrow}(A)} u_{i} u_{i}^{\top}\right)\left(\sum_{i \in S} \lambda_{i}^{\downarrow}(A) u_{i} u_{i}^{\top}\right)=A^{+} A \\
& =\sum_{i \in S} u_{i} u_{i}^{\top}=P_{\operatorname{Im}(A)} .
\end{aligned}
$$

\subsection{Grafos}

Nesta seção, mostraremos algumas definições relacionadas a grafos, que serão utilizadas ao longo do texto. Primeiramente, dizemos que um par $(V, E)$ é um grafo se $V$ é um conjunto e $E \subseteq\left(\begin{array}{c}V \\ 2\end{array}\right)$. Chamamos $V$ e $E$ de conjuntos de vértices e arestas do grafo, respectivamente. Dizemos também que um grafo possui pesos nas arestas se este é associado a uma função de peso $w: E \rightarrow \mathbb{R}_{+}$, que atribui um valor real não-negativo a cada uma das arestas do grafo em questão. Representamos o grafo $(V, E)$ associado a uma função peso $w$ nas arestas como a tripla $(V, E, w)$.

Seja $G=(V, E)$ um grafo e sejam $v_{1}, \ldots, v_{m} \in V$. Dizemos que $\left(v_{1}, v_{2}, \ldots, v_{m}\right)$ é um passeio de $v_{1}$ a $v_{m}$ em $G$ se $\left\{v_{i}, v_{i+1}\right\} \in E$ para todo $i \in[m-1]$. Além disso, se $v_{i} \neq v_{j}$ para todo $i, j \in[m]$ com $i \neq j$, então dizemos que $\left(v_{1}, v_{2}, \ldots, v_{m}\right)$ é um caminho de $v_{1}$ a $v_{m}$ em $G$ de comprimento $m-1$. Denotamos por origem e término de um passeio o primeiro e o último vértice desse passeio, respectivamente. Também denotamos por distância entre dois vértices em um grafo $G$ o comprimento de um caminho de comprimento mínimo que contém esses dois vértices em $G$. Caso não haja caminho entre dois vértices, a distância entre eles é $+\infty$. Utilizamos a notação $\operatorname{dist}_{G}(u, v)$ para nos referir a distância entre os vértices $u$ e $v$ no grafo $G$.

Seja $G=(V, E)$ um grafo e $p:=\left(v_{1}, v_{2}, \ldots, v_{m}\right)$ um passeio em $G$. Defina o conjunto de passeios que são extensões de $p$ como

$$
P_{G, p}:=\left\{\left(v_{1}, v_{2}, \ldots, v_{m}, u\right): u \in \Gamma_{G}\left(v_{m}\right)\right\} .
$$

Se $p$ é um caminho, então defina o conjunto de caminhos que são extensões de $p$ como

$$
C_{G, p}:=\left\{\left(v_{1}, v_{2}, \ldots, v_{m}, u\right): u \in \Gamma_{G}\left(v_{m}\right) \backslash\left\{v_{1}, \ldots, v_{m}\right\}\right\} .
$$

Utilizamos essas definições de conjuntos de extensões para apresentar as coberturas universais e as árvores de caminhos.

Definição 2.45 (Cobertura universal). Seja $G=(V, E)$ um grafo e $v \in V$. Defina a cobertura universal de $G$ como o grafo $T_{G}:=\left(V_{1}, E_{1}\right)$, onde $V_{1}$ é o conjunto de passeios com origem $v$, e

$$
E_{1}:=\left\{\{u, w\} \in\left(\begin{array}{c}
V_{1} \\
2
\end{array}\right): u \in P_{G, w} \text { ou } w \in P_{G, u}\right\} .
$$

Pela definição acima, é fácil ver que todas as coberturas universais de um grafo conexo são isomorfas. Por esse motivo, não incluímos o vértice $v$ na notação. 
Seja $G=(V, E)$ um grafo e $v \in V$. Defina também a árvore de caminhos de $G$ com raiz $v$ como $P(G, v):=\left(V_{2}, E_{2}\right)$, onde $V_{2}$ é o conjunto de caminhos com origem $v$ e $E_{2}:=\left\{\{u, w\} \in\left(\begin{array}{c}V_{2} \\ 2\end{array}\right)\right.$ : $u \in C_{G, w}$ ou $\left.w \in C_{G, u}\right\}$. Denote $P(G, v)-v:=P(G, v)\left[V_{2} \backslash\{(v)\}\right]$, onde $(v)$ é o caminho de tamanho 0 que contém apenas o vértice $v$. Seguindo a lógica de nomenclatura de árvores de caminhos, faz sentido chamar coberturas universais de árvores de passeios.

Definição 2.46 (União de grafos). Sejam $G=\left(V_{1}, E_{1}\right)$ e $H=\left(V_{2}, E_{2}\right)$ grafos. Dizemos que $G \cup H$ é o grafo $\left(V_{1} \cup V_{2}, E_{1} \cup E_{2}\right)$.

Lema 2.47. Seja $G=(V, E)$ um grafo e $v \in V$. Então $P(G, v)-v=\bigcup_{u \in \Gamma_{G}(v)} P(G-v, u)$.

Demonstração. Como $P(G, v)$ é uma árvore, então vale que $\operatorname{dist}_{P(G, v)-v}(u, w)=+\infty$ para todo par $u, w \in \Gamma_{P(G, v)}((v))$ com $u \neq w$, o que implica que $P(G, v)-v=\bigcup_{u \in \Gamma_{G}(v)} P(G-v, u)$.

Lema 2.48. Seja $G=(V, E)$ uma árvore com $|V| \geq 2$. Então existe $v \in V$ tal que $\operatorname{grau}_{G}(v)=1$.

Demonstração. Seja $n:=|V|$. Note que $|E|=n-1$, o que implica que $\sum_{v \in V} \operatorname{grau}_{G}(v)=2 n-2$. Portanto, existe $v \in V$ tal que $\operatorname{grau}_{G}(v)<2$. Como $G$ é conexo e $|V| \geq 2$, então $\operatorname{grau}_{G}(u) \geq 1$ para todo $u \in V$. Logo, vale que $\operatorname{grau}_{G}(v)=1$.

Seja $G=(V, E)$ um grafo e sejam $U_{1}, U_{2} \subseteq V$ disjuntos. Dizemos que $G$ é $\left(U_{1}, U_{2}\right)$-bipartido se $U_{1} \cup U_{2}=V$ e se $\left|e \cap U_{1}\right|=\left|e \cap U_{2}\right|=1$ para todo $e \in E$. Além disso, se grau $(u)=c$ para todo $u \in U_{1}$ e $\operatorname{grau}(u)=d$ para todo $u \in U_{2}$, então dizemos que $G$ é $(c, d)$-birregular.

Definição 2.49 (Grafos de Cayley). Seja $G$ um grupo e $S \subseteq G$ tal que $S=S^{-1}$. Defina o grafo de Cayley $\Gamma(G, S)$ como o grafo $(G, E)$, onde $E:=\{\{g, g h\}: g \in G, h \in S\}$.

Dizemos que o par $D=(V, A)$ é um digrafo se $V$ é um conjunto finito e $A \subseteq V \times V$. Denotamos por conjuntos de vértices e arcos os conjuntos $V$ e $A$, respectivamente. Seja $D=(V, A)$ um digrafo. Defina a matriz de incidência de $D$ como $B_{D} \in\{-1,0,1\}^{V \times A}$ tal que

$$
B_{D}(v,(u, w))=\left\{\begin{aligned}
-1, & \text { se } v=u \\
+1, & \text { se } v=w \\
0, & \text { caso contrário }
\end{aligned}\right.
$$

para todo $v \in V$ e todo $(u, w) \in A$.

Seja $G=(V, E, w)$ um grafo com pesos nas arestas e $\vec{G}=(V, A)$ um digrafo. Dizemos que $\vec{G}$ é uma orientação de $G$ se existe uma função bijetora $f: A \rightarrow E$ tal que $f(u, v)=\{u, v\}$ para quaisquer $(u, v) \in A$. Dizemos também que $G$ é o grafo subjacente de $\vec{G}$.

Seja $D=(V, A)$ um digrafo. Dizemos que $\left(v_{1}, v_{2}, \ldots, v_{m}\right)$ é um caminho em $D$ de $v_{1}$ a $v_{m}$ de comprimento $m-1$ se $\left(v_{i}, v_{i+1}\right) \in A$ para todo $i \in[m-1]$. Denotamos por origem e término de um caminho o primeiro e o último vértices desse caminho. Dizemos que a distância entre dois vértices $u$ e $v$ em $D$ é o comprimento de um caminho em $D$ de comprimento mínimo cuja origem é o vértice $u$ e o término, o vértice $v$. Denotamos por $\operatorname{dist}_{D}(u, v)$ a distância entre os vértices $u$ e $v$ no digrafo $D$. Defina

$$
\delta_{\text {in }}(v):=\left\{\left(a_{1}, a_{2}\right) \in A: a_{2}=v\right\} \quad \text { e } \quad \delta_{\text {out }}(v):=\left\{\left(a_{1}, a_{2}\right) \in A: a_{1}=v\right\}
$$

para todo $v \in V$. Defina também

$$
N_{\text {in }}(v):=\{u \in V:(u, v) \in A\} \quad \text { e } \quad N_{\text {out }}(v):=\{u \in V:(v, u) \in A\}
$$

para todo $v \in V$. 
Seja $T=(V, A)$ um digrafo tal que o grafo subjacente de $T$ é uma árvore. Dizemos que $T$ é uma arborescência se

$$
\left|\left\{v \in V:\left|\delta_{\text {in }}(v)\right|=0\right\}\right|=1 \quad \text { e } \quad\left|\left\{v \in V:\left|\delta_{\text {in }}(v)\right|=1\right\}\right|=|V|-1 .
$$

Defina a raiz da arborescência $T$ como $r(T):=v$, onde $v \in V$ é o único vértice que satisfaz $\left|\delta_{\text {in }}(v)\right|=0$. Seja $T=(V, A)$ uma arborescência. Dizemos que $v$ é um nó de $T$ se $v \in V$. Seja $v$ um nó de $T$. Dizemos que $v$ é um nó interno de $T$ se $N_{\text {out }}(v) \neq \varnothing$ e que $v$ é uma folha, caso contrário. Defina o conjunto de descendentes de $v$ em $T$ como $\{u \in V$ : existe um caminho de $v$ a $u\}$. Defina também a altura de $v$ em $T$ como

$$
h_{T}(v):=\max \left\{\operatorname{dist}_{T}(v, u): \text { existe caminho de } v \text { até } u \text { em } T\right\}
$$

e a altura de $T$ como $h(T):=h_{T}(r(T))$.

\subsection{Teoria espectral dos grafos}

Teorema 2.50 (Autovalores de grafos bipartidos). Seja $G$ um grafo bipartido com $n$ vértices. Então

$$
\lambda_{i}^{\downarrow}\left(A_{G}\right)=-\lambda_{i}^{\uparrow}\left(A_{G}\right)
$$

para todo $i \in[n]$.

Demonstração. Para provar esse resultado, basta mostrar que $-\lambda_{i}^{\downarrow}\left(A_{G}\right)$ é um autovalor de $A_{G}$ para todo $i \in[n]$.

Sejam $U, V \subseteq V(G)$ não vazios, disjuntos e tais que $U \cup V=V(G)$ e $|e \cap U|=|e \cap V|=1$ para todo $e \in E(G)$. Defina $B:=A_{G}[V, U]$. Note que

$$
A_{G}=\left[\begin{array}{cc}
0 & B^{\top} \\
B & 0
\end{array}\right] .
$$

Seja $i \in[n]$. Sejam $u \in \mathbb{R}^{U}$ e $v \in \mathbb{R}^{V}$ tais que $u \oplus v$ é um autovetor de $A_{G}$ associado a $\lambda_{i}^{\downarrow}\left(A_{G}\right)$. Assim, vale que $B^{\top} v=\lambda_{i}^{\downarrow}\left(A_{G}\right) u$ e $B u=\lambda_{i}^{\downarrow}\left(A_{G}\right) v$. Dessa forma, vale que $B^{\top}(-v)=\left(-\lambda_{i}^{\downarrow}\left(A_{G}\right)\right) u$ e $B u=\left(-\lambda_{i}^{\downarrow}\left(A_{G}\right)\right)(-v)$. Logo

$$
A_{G}(u \oplus-v)=\left(-\lambda_{i}^{\downarrow}\left(A_{G}\right)\right)(u \oplus-v)
$$

e, portanto, vale que $-\lambda_{i}^{\downarrow}\left(A_{G}\right)$ é um autovalor de $A_{G}$.

Lema 2.51. Seja $G$ um grafo. Então $\rho(G) \leq \Delta(G)$.

Demonstração. Sejam $\lambda \in \mathbb{R}$ e $v \in \mathbb{R}^{V(G)} \backslash\{0\}$ tais que $A_{G} v=\lambda v$. Tome $v_{\max }:=\max _{i \in V(G)}\left|v_{i}\right|>0$. Note que, para todo $i \in V(G)$, vale que

$$
\begin{aligned}
|\lambda|\left|v_{i}\right| & =\left|(\lambda v)_{i}\right|=\left|\left(A_{G} v\right)_{i}\right| \\
& =\left|\sum_{j \in \Gamma_{G}(i)} v_{j}\right| \leq \sum_{j \in \Gamma_{G}(i)}\left|v_{j}\right| \\
& \leq\left|\Gamma_{G}(i)\right| v_{\max } \leq \Delta(G) v_{\max } .
\end{aligned}
$$

Logo, $|\lambda| v_{\max } \leq \Delta(G) v_{\max }$, o que implica que $|\lambda| \leq \Delta(G)$. Portanto, $\rho(G) \leq \Delta(G)$. 
Corolário 2.52. Seja $G$ um grafo $d$-regular. Então, $\lambda_{1}^{\downarrow}\left(A_{G}\right)=d$.

Demonstração. Note que $A_{G} \mathbb{1}=d \mathbb{1}$, logo $\mathbb{1}$ é um autovetor de $A_{G}$. Portanto, $\lambda_{1}^{\downarrow}\left(A_{G}\right) \geq d$. Pelo Lema 2.51, vale que $\left|\lambda_{1}^{\downarrow}\left(A_{G}\right)\right| \leq \Delta(G)=d$, o que implica que $\lambda_{1}^{\downarrow}\left(A_{G}\right)=d$.

Lema 2.53. Seja $G$ um grafo regular conexo com $n$ vértices. Então $G$ é bipartido se e somente se

$$
\lambda_{1}^{\downarrow}\left(A_{G}\right)=-\lambda_{n}^{\downarrow}\left(A_{G}\right) .
$$

Demonstração. Suponha que $G$ é bipartido. Pelo Teorema 2.50, temos que $\lambda_{1}^{\downarrow}\left(A_{G}\right)=-\lambda_{n}^{\downarrow}\left(A_{G}\right)$.

Suponha agora que $\lambda_{1}^{\downarrow}\left(A_{G}\right)=-\lambda_{n}^{\downarrow}\left(A_{G}\right)$. Seja $d$ a valência de $G$ e seja $V:=V(G)$. Pelo Corolário 2.52, temos que $\lambda_{n}^{\downarrow}\left(A_{G}\right)=-d$. Seja $x \in \mathbb{R}^{V} \backslash\{0\}$ um autovetor de $A_{G}$ associado ao autovalor $-d$. Assim, vale que $A_{G} x=-d x$.

Seja $u \in V$ tal que $0 \neq\left|x_{u}\right| \geq\left|x_{w}\right|$ para todo $w \in V$. Logo, vale que

$$
-d x_{u}=\left(\left(A_{G}\right) x\right)_{u}=e_{u}^{\top} A_{G} x=\sum_{v \in \Gamma_{G}(u)} x_{v} .
$$

Como $\left|\Gamma_{G}(u)\right|=d$ e $\left|x_{u}\right| \geq\left|x_{w}\right|$ para todo $w \in V$, então vale que $x_{w}=-x_{u}$ para todo $w \in \Gamma_{G}(u)$. Logo, se $u \in V \operatorname{com}\left|x_{u}\right| \geq\left|x_{w}\right|$ para todo $w \in V$, então vale que $x_{v}=-x_{u}$ para todo $v \in \Gamma_{G}(u)$. Portanto, como $G$ é conexo, vale que $\left|x_{u}\right|=\left|x_{v}\right|$ para todos $u, v \in V$, o que implica que $x_{u} \neq 0$ para todo $u \in V$.

Defina $S_{1}:=\left\{v \in V: x_{v}>0\right\}$ e $S_{2}:=\left\{v \in V: x_{v}<0\right\}$. Note que $S_{1} \cap S_{2}=\varnothing$. Como $x_{u} \neq 0$ para todo $u \in V$, então $S_{1} \cup S_{2}=V$. Note que $\left|e \cap S_{1}\right|=\left|e \cap S_{2}\right|=1$ para todo $e \in E(G)$. Portanto, vale que $G$ é bipartido.

Proposição 2.54 (Espectro de grafos completos). Seja $n \in \mathbb{N}$ com $n \geq 2$. Então

$$
\lambda^{\downarrow}\left(A_{K_{n}}\right)=n e_{1}-\mathbb{1} .
$$

Demonstração. Denote $K:=K_{n}$. Seja $J \in\{1\}^{n \times n}$. Note que $A_{K}=J-I$. Assim, vale que $\lambda_{i}^{\downarrow}\left(A_{K}\right)=\lambda_{i}^{\downarrow}(J)-1$. Note que $\mathbb{1}$ é um autovalor de $J$ associado ao autovalor $n$. Assim, pela Proposição 2.40 , temos que

$$
\lambda_{2}^{\downarrow}(J)=\cdots=\lambda_{n}^{\downarrow}(J)=0 .
$$

Portanto, vale que $\lambda_{1}^{\downarrow}\left(A_{K}\right)=n-1$ e $\lambda_{i}^{\downarrow}\left(A_{K}\right)=-1$ para todo $i \in\{2,3, \ldots, n\}$.

Proposição 2.55 (Espectro de grafos bipartidos completos). Seja $m, n \in \mathbb{N} \operatorname{com} m, n \geq 2$. Então

$$
\lambda^{\downarrow}\left(A_{K_{m, n}}\right)=\sqrt{m n} e_{1}-\sqrt{m n} e_{m+n} .
$$

Demonstração. Denote $K:=K_{m, n}$. Sejam

$$
M:=\{v \in V(K): \operatorname{grau}(v)=n\} \text { e } N:=\{v \in V(K): \operatorname{grau}(v)=m\} .
$$

Note que $|M|=m$ e $|N|=n$. Seja $v=\sqrt{m} \mathbb{1}_{N}+\sqrt{n} \mathbb{1}_{M}$. Note que $A_{K} v=\sqrt{m n} v$. Assim, vale que $\sqrt{m n} \in \operatorname{Spec}\left(A_{K}\right)$. Logo, pelo Lema 2.53, temos que $-\sqrt{m n} \in \operatorname{Spec}\left(A_{K}\right)$.

Note que $\operatorname{rank}\left(A_{K}\right)=2$. Assim, pela Proposição 2.40, temos que

$$
\begin{gathered}
\lambda_{1}^{\downarrow}\left(A_{K}\right)=-\lambda_{m+n}^{\downarrow}\left(A_{K}\right)=\sqrt{m n}, \\
\lambda_{2}^{\downarrow}\left(A_{K}\right)=\cdots=\lambda_{m+n-1}^{\downarrow}\left(A_{K}\right)=0 .
\end{gathered}
$$


Lema 2.56. Sejam $G=(V, E)$ um grafo e $v \in V$ tal que $\operatorname{grau}_{G}(v)=1$. Seja $z \in \Gamma_{G}(v)$. Então

$$
p_{A_{G}}(x)=x \operatorname{det}\left(x I-A_{G-v}\right)-\operatorname{det}\left(x I-A_{G-v-z}\right),
$$

onde $x$ é uma indeterminada.

Demonstração. Defina $n:=|V|$. Podemos supor que $V=[n]$. Defina $B:=x I-A_{G}$. Note que

$$
\begin{aligned}
\operatorname{det}\left(x I-A_{G}\right) & =\operatorname{det}(B) \\
& =\sum_{u \in V}(-1)^{v+u} B_{v, u} \operatorname{det}(B[V \backslash\{v\}, V \backslash\{u\}]) .
\end{aligned}
$$

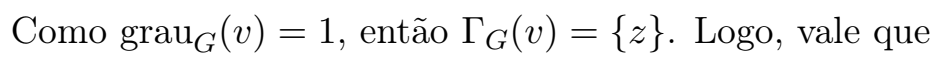

$$
B_{u, v}=B_{v, u}= \begin{cases}x, & \text { se } u=v \\ -1, & \text { se } u=z \\ 0, & \text { caso contrário. }\end{cases}
$$

Assim, vale que

$$
\operatorname{det}\left(x I-A_{G}\right)=x \operatorname{det}(B[V \backslash\{v\}, V \backslash\{v\}])+(-1)^{v+z}(-1) \operatorname{det}(B[V \backslash\{v\}, V \backslash\{z\}]) .
$$

Note que

$$
\begin{aligned}
\operatorname{det}(B & {[V \backslash\{v\}, V \backslash\{z\}]) } \\
& =\sum_{u \in V \backslash\{v\}}(-1)^{u+v-1} B_{u, v} \operatorname{det}(B[V \backslash\{v, u\}, V \backslash\{z, v\}]) \\
& =(-1)^{z+v-1}(-1) \operatorname{det}\left(x I-A_{G-v-z}\right) .
\end{aligned}
$$

Note também que

$$
\operatorname{det}(B[V \backslash\{v\}, V \backslash\{v\}])=\operatorname{det}\left(x I-A_{G-v}\right) .
$$

Assim, por (2.5), (2.6) e (2.7), temos que

$$
\begin{aligned}
\operatorname{det}\left(x I-A_{G}\right) & =(-1)^{v+v} x \operatorname{det}\left(x I-A_{G-v}\right)+(-1)^{v+z}(-1) \operatorname{det}(B[V \backslash\{v\}, V \backslash\{z\}]) \\
& =x \operatorname{det}\left(x I-A_{G-v}\right)+(-1)^{v+z}(-1)(-1)^{z+v} \operatorname{det}\left(x I-A_{G-v-z}\right) \\
& =x \operatorname{det}\left(x I-A_{G-v}\right)-\operatorname{det}\left(x I-A_{G-v-z}\right) .
\end{aligned}
$$

Definição 2.57 (Laplaciano). Sejam $G=(V, E, w)$ um grafo com pesos nas arestas e $\vec{G}=(V, A)$ uma orientação de $G$. Defina $w^{\prime}: A \rightarrow \mathbb{R}$ como $(i, j) \mapsto w(\{i, j\})$. Dizemos que

$$
L_{G}:=B_{\vec{G}} \operatorname{Diag}\left(w^{\prime}\right) B_{\vec{G}}^{\top}
$$

é o Laplaciano de $G$. 


\subsection{Polinômios de emparelhamentos}

Nesta seção, iremos definir e mostrar algumas características de polinômios de emparelhamentos, que são polinômios univariados associados a grafos. Cada coeficiente de um polinômio de emparelhamentos associado a um grafo representa o número de emparelhamentos de um certo tamanho nesse grafo. Caso uma função de peso nas arestas esteja associada ao grafo, esses coeficientes terão os pesos de cada aresta do grafo levados em consideração. Na maioria dos casos, não consideraremos polinômios de emparelhamentos de grafos com pesos nas arestas. Entretanto, precisaremos dos pesos para provar que suas raízes são todas reais usando um argumento de continuidade.

Esses polinômios são utilizados em química e física para o cálculo da atividade de monômeros em um sistema de monômeros e dímeros, sendo monômero uma molécula, e dímero, um composto de dois monômeros. Neste caso, o sistema de monômeros e dímeros é representado por um grafo, cujos vértices e arestas são representações dos monômeros e dos possíveis dímeros do sistema, respectivamente.

Uma de suas características, que será mostrada nesta seção, é que, se considerarmos uma árvore, o seu polinômio de emparelhamentos é igual ao polinômio característico da sua matriz de adjacência. Outra característica desses polinômios é que todas as suas raízes são reais, e quando o grafo é $d$-regular, com $d>1$, suas raízes são limitadas superiormente por $2 \sqrt{d-1}$. Essa característica, juntamente com algumas ferramentas, nos permitirá utilizar esses polinômios para provar a existência de bons grafos expansores, como mostraremos no Capítulo 6.

Definição 2.58. Seja $G=(V, E)$ um grafo. Seja $w$ uma função tomando valores em $\mathbb{R}_{+}$cujo domínio contém $E$. Denote por $\mathcal{M}_{G}(d)$ o conjunto dos emparelhamentos de $G$ com $d$ arestas para todo $d \in \mathbb{N}$. Defina

$$
\mathrm{m}_{G, \boldsymbol{w}}(d):=\sum_{M \in \mathcal{M}_{G}(d)} \prod_{e \in M} w_{e}
$$

para todo $d \in \mathbb{N}$.

Definição 2.59 (Polinômio de emparelhamentos). Seja $G=(V, E)$ um grafo com $n$ vértices. Seja $w$ uma função tomando valores em $\mathbb{R}_{+}$cujo domínio contém $E$. Defina o polinômio de emparelhamentos do grafo $G$ com pesos $w$ como

$$
\mu_{G, \boldsymbol{w}}(x):=\sum_{d \in \mathbb{N}} x^{n-2 d}(-1)^{d} \mathrm{~m}_{G, \boldsymbol{w}}(d) \in \mathbb{R}[x] .
$$

Defina também $\mu_{G}(x):=\mu_{G, \mathbb{1}}(x)$.

Note que o polinômio de emparelhamentos de qualquer grafo possui coeficiente dominante igual a 1, dado que todo grafo possui um único emparelhamento de tamanho zero. Note também que, se considerarmos um grafo $G$ com peso 1 nas arestas, então $\mathrm{m}_{G, \mathbb{1}}(d)$ é o número de emparelhamentos em $G$ com $d$ arestas, onde $d \in \mathbb{N}$. Por exemplo, o polinômio de emparelhamentos do grafo bipartido $K_{3,3}$ com peso $\mathbb{1}$ nas arestas é

$$
\mu_{K_{3,3}}(x)=x^{6}-9 x^{4}+18 x^{2}-6 .
$$

É fácil ver que os valores absolutos dos coeficientes dos monômios $x^{6}, x^{4}, x^{2}$ e $x^{0}$ de $\mu_{K_{3,3}}$ são os números de emparelhamentos em $K_{3,3}$ com zero, uma, duas e três arestas, respectivamente.

Também é possível escrever um polinômio de emparelhamentos de um grafo $G$ com peso $w$ nas arestas como uma fórmula de recorrência, utilizando os polinômios de emparelhamentos de alguns dos subgrafos induzidos de $G$. Essa recorrência será utilizada neste texto para provar por indução algumas propriedades dos polinômios de emparelhamentos. Para provar essa recorrência, utilizaremos uma fórmula de recorrência para $\mathrm{m}_{G, \boldsymbol{w}}$. 
Proposição 2.60. Sejam $G=(V, E, w)$ um grafo com pesos nas arestas e $v \in V$. Então

$$
\mathrm{m}_{G, \boldsymbol{w}}(d)=\mathrm{m}_{G-v, \boldsymbol{w}}(d)+\sum_{u \in \Gamma_{G}(v)} w(\{u, v\}) \mathrm{m}_{G-v-u, \boldsymbol{w}}(d-1)
$$

para todo $d \in \mathbb{N} \backslash\{0\}$.

Demonstração. Seja $d \in \mathbb{N} \backslash\{0\}$. Defina

$$
\mathcal{N}_{1}:=\left\{M \in \mathcal{M}_{G}(d): \delta_{G}(v) \cap M=\varnothing\right\} \quad \text { e } \quad \mathcal{N}_{2}:=\left\{M \in \mathcal{M}_{G}(d): \delta_{G}(v) \cap M \neq \varnothing\right\} .
$$

Note que $\mathcal{N}_{1} \cap \mathcal{N}_{2}=\varnothing$ e $\mathcal{N}_{1} \cup \mathcal{N}_{2}=\mathcal{M}_{G}(d)$. Pela definição de $\mathcal{N}_{1}$, é fácil ver que $\mathcal{N}_{1}=\mathcal{M}_{G-v}(d)$. Além disso, como $\left|M \cap \delta_{G}(v)\right|=1$ para todo $M \in \mathcal{N}_{2}$, então

$$
\mathcal{N}_{2}=\bigcup_{u \in \Gamma_{G}(v)}\left\{M \cup\{\{u, v\}\}: M \in \mathcal{M}_{G-v-u}(d-1)\right\}
$$

Assim, vale que

$$
\sum_{M \in \mathcal{N}_{1}} \prod_{e \in M} w_{e}=\sum_{M \in \mathcal{M}_{G-v}(d)} \prod_{e \in M} w_{e}=\mathrm{m}_{G-v, \boldsymbol{w}}(d)
$$

Além disso, como a família de emparelhamentos $\left\{M \cup\{\{u, v\}\}: M \in \mathcal{M}_{G-v-u}(d-1)\right\}_{u \in \Gamma_{G}(v)}$ é disjunta dois a dois, então

$$
\begin{aligned}
\sum_{M \in \mathcal{N}_{2}} \prod_{e \in M} w_{e} & =\sum_{u \in \Gamma_{G}(v)} w(\{u, v\}) \sum_{M \in \mathcal{M}_{G-v-u}(d-1)} \prod_{e \in M} w_{e} \\
& =\sum_{u \in \Gamma_{G}(v)} w(\{u, v\}) \mathrm{m}_{G-v-u, \boldsymbol{w}}(d-1) .
\end{aligned}
$$

Portanto, por (2.9) e (2.10), vale que

$$
\begin{aligned}
\mathrm{m}_{G, \boldsymbol{w}}(d) & =\sum_{M \in \mathcal{M}_{G}(d)} \prod_{e \in M} w_{e} \\
& =\sum_{M \in \mathcal{N}_{1}} \prod_{e \in M} w_{e}+\sum_{M \in \mathcal{N}_{2}} \prod_{e \in M} w_{e} \\
& =\mathrm{m}_{G-v, \boldsymbol{w}}(d)+\sum_{u \in \Gamma_{G}(v)} w(\{u, v\}) \mathrm{m}_{G-v-u, \boldsymbol{w}}(d-1) .
\end{aligned}
$$

Teorema 2.61 (vide [16]). Sejam $G=(V, E, w)$ um grafo com pesos nas arestas e $v \in V$. Então

$$
\mu_{G, \boldsymbol{w}}(x)=x \mu_{G-v, \boldsymbol{w}}(x)-\sum_{u \in \Gamma_{G}(v)} w(\{u, v\}) \mu_{G-v-u, \boldsymbol{w}}(x) .
$$

Demonstração. Defina $n:=|V|$. Defina também $\Gamma(v):=\Gamma_{G}(v)$ para todo $v \in V$. Pela Proposi- 
ção 2.60 , temos que

$$
\begin{aligned}
\mu_{G, \boldsymbol{w}}(x)= & \sum_{d \in \mathbb{N}} x^{n-2 d}(-1)^{d} \mathrm{~m}_{G, \boldsymbol{w}}(d) \\
= & x^{n}+\sum_{d \in \mathbb{N} \backslash\{0\}} x^{n-2 d}(-1)^{d}\left(\mathrm{~m}_{G-v, \boldsymbol{w}}(d)+\sum_{u \in \Gamma(v)} w(\{u, v\}) \mathrm{m}_{G-v-u, \boldsymbol{w}}(d-1)\right) \\
= & x^{n}+\sum_{d \in \mathbb{N} \backslash\{0\}} x^{n-2 d}(-1)^{d} \mathrm{~m}_{G-v, \boldsymbol{w}}(d)+\sum_{d \in \mathbb{N} \backslash\{0\}} x^{n-2 d}(-1)^{d} \sum_{u \in \Gamma(v)} w(\{u, v\}) \mathrm{m}_{G-v-u, \boldsymbol{w}}(d-1) \\
= & \sum_{d \in \mathbb{N}} x^{n-2 d}(-1)^{d} \mathrm{~m}_{G-v, \boldsymbol{w}}(d)+\sum_{u \in \Gamma(v)} \sum_{d \in \mathbb{N} \backslash\{0\}} x^{n-2 d}(-1)^{d} w(\{u, v\}) \mathrm{m}_{G-v-u, \boldsymbol{w}}(d-1) \\
= & x \mu_{G-v, \boldsymbol{w}}(x)+\sum_{u \in \Gamma(v)} w(\{u, v\}) \sum_{d \in \mathbb{N} \backslash\{0\}} x^{n-2 d}(-1)^{d} \mathrm{~m}_{G-v-u, \boldsymbol{w}}(d-1) .
\end{aligned}
$$

Note também que, para todo $u \in \Gamma(v)$, vale que

$$
\begin{aligned}
\sum_{d \in \mathbb{N} \backslash\{0\}} x^{n-2 d} & (-1)^{d} \mathrm{~m}_{G-v-u, \boldsymbol{w}}(d-1) \\
& =\sum_{d \in \mathbb{N}} x^{n-2(d+1)}(-1)^{(d+1)} \mathrm{m}_{G-v-u, \boldsymbol{w}}(d+1-1) \\
& =-\mu_{G-v-u, \boldsymbol{w}}(x)
\end{aligned}
$$

Portanto, o resultado segue de (2.11) e (2.12).

Utilizaremos novamente o grafo bipartido completo $K_{3,3}$ para exemplificar a fórmula de recorrência mostrada no Teorema 2.61. Sejam $u_{1}, u_{2}, u_{3}, v_{1}, v_{2}, v_{3}$ os vértices de $K_{3,3}$ tais que $\left\{u_{i}, v_{j}\right\} \in$ $E\left(K_{3,3}\right)$ para todo $i, j \in\{1,2,3\}$. Note que o polinômio de emparelhamentos de $K_{3,3}-u_{3}$ é $\mu_{K_{3,3}-u_{3}}(x)=x^{5}-6 x^{3}+6 x$. Note também que, se retirarmos qualquer um dos vértices $v_{i} \in \Gamma_{K_{3,3}}\left(u_{3}\right)$ do grafo $K_{3,3}-u_{3}$, obteremos grafos isomorfos ao $K_{2,2}$, o que implica que

$$
\mu_{K_{3,3}-u_{3}-v_{1}}(x)=\mu_{K_{3,3}-u_{3}-v_{2}}(x)=\mu_{K_{3,3}-u_{3}-v_{3}}(x)=x^{4}-4 x^{2}+2 .
$$

Assim, pelo Teorema 2.61, temos que

$$
\begin{aligned}
\mu_{K_{3,3}}(x) & =x \mu_{K_{3,3}-u_{3}}(x)-\sum_{i \in[3]} \mu_{K_{3,3}-u_{3}-v_{i}}(x) \\
& =x\left(x^{5}-6 x^{3}+6 x\right)-3\left(x^{4}-4 x^{2}+2\right) \\
& =x^{6}-9 x^{4}+18 x^{2}-6,
\end{aligned}
$$

como mostrado em (2.8).

Vamos provar agora que, se considerarmos uma árvore, seu polinômio de emparelhamentos é igual ao polinômio característico da sua matriz de adjacência. Esse resultado será utilizado no Capítulo 6 para provar que as raízes do polinômio de emparelhamentos de um grafo conexo $G$ são limitadas superiormente pelo raio espectral da matriz de adjacência da cobertura universal de $G$.

Teorema 2.62. Seja $G=(V, E)$ uma floresta com $|V| \geq 1$. Então $\mu_{G}(x)=p_{A_{G}}(x)$. 
Demonstração. Defina $n:=|V|$. Provaremos este resultado por indução em $n$.

Se $|E|=0$, então $\mu_{G}(x)=x^{n}=\operatorname{det}\left(x I-A_{G}\right)$. Suponha que $|E|>0$.

Se $n=1$, então $|E|=0$. Se $n=2$, então $\mu_{G}(x)=x^{2}-1=\operatorname{det}\left(x I-A_{G}\right)$.

Suponha que $n \geq 3$. Como $|E|>0$, então, pelo Lema 2.48, existe $v \in V$ tal que $\operatorname{grau}_{G}(v)=1$. Seja $u \in \Gamma_{G}(v)$. Pelo Teorema 2.61, temos que

$$
\mu_{G}(x)=x \mu_{G-v}(x)-\mu_{G-v-u}(x) .
$$

Pela hipótese de indução, temos que $\mu_{G-v}(x)=\operatorname{det}\left(x I-A_{G-v}\right)$, e também que $\mu_{G-v-u}(x)=$ $\operatorname{det}\left(x I-A_{G-v-u}\right)$, o que implica que

$$
\mu_{G}(x)=x \operatorname{det}\left(x I-A_{G-v}\right)-\operatorname{det}\left(x I-A_{G-v-u}\right) .
$$

Portanto, pelo Lema 2.56, temos que $\mu_{G}(x)=\operatorname{det}\left(x I-A_{G}\right)$. 


\section{Capítulo 3}

\section{Grafos expansores}

Neste capítulo, definiremos formalmente grafos expansores utilizando um parâmetro de grafos chamado índice de expansão e utilizando também o conceito de famílias infinitas de grafos $d$-regulares para garantir um número linear de arestas em relação ao número de vértices. Discutiremos algumas propriedades de grafos expansores: eles formam um único cluster, se comportam como grafos aleatórios em alguns casos, e a distribuição de probabilidade de seus passeios aleatórios converge rapidamente para a distribuição uniforme. Mostraremos também que existe um limitante superior assintótico para o índice de expansão de grafos $d$-regulares, motivando assim a introdução dos grafos de Ramanujan, que são grafos que atingem esse limitante.

O índice de expansão de um grafo $G=(V, E)$, denotado por $h(G)$, é a menor das proporções entre o tamanho de um corte de $G$ e o tamanho de sua menor margem. Assim, para todo $S \subseteq V$ com $0<|S| \leq \frac{|V|}{2}$, a proporção entre o tamanho do corte $\delta(S)$ e o tamanho de $S$ é pelo menos o índice de expansão de $G$. Em outras palavras, para separar $k$ vértices do restante do grafo, é preciso remover pelo menos $h(G) k$ arestas. Estamos interessados em grafos com um índice de expansão grande, mais precisamente, sequências de grafos de tamanho crescente em que o índice de expansão não converge para zero.

Um conceito relacionado é o índice de expansão dos vértices de um grafo $G=(V, E)$, que é a menor das proporções entre o tamanho da vizinhança de um subconjunto $S$ de vértices e o tamanho de $S$, com $0<|S| \leq|\bar{S}|$. Dessa forma, para todo $S \subseteq V$ com $0<|S| \leq \frac{|V|}{2}$, a proporção entre $\Gamma_{G}(S)$ e $|S|$ é pelo menos o índice de expansão dos vértices de $G$. Em outras palavras, o índice de expansão dos vértices indica o número de vértices que devem ser removidos para desconectar qualquer subconjunto de vértices de acordo com o tamanho desse subconjunto. Como estamos nos referindo a grafos simples, é fácil ver que o índice de expansão dos vértices é um limitante inferior para o índice de expansão das arestas, apresentado no parágrafo anterior. Porém, neste texto, estamos interessados apenas no índice de expansão das arestas.

Definição 3.1 (Índice de expansão). Seja $G=(V, E)$ um grafo com $n$ vértices. Defina o índice de expansão de $G$ como

$$
h(G):=\min \left\{\frac{|\delta(S)|}{|S|}: \varnothing \neq S \subseteq V,|S| \leq \frac{n}{2}\right\} .
$$

Note que, para todo grafo $G=(V, E)$ com $n$ vértices, vale que

$$
h(G) \leq \min \left\{\frac{|S|(n-|S|)}{|S|}: \varnothing \neq S \subseteq V,|S| \leq \frac{n}{2}\right\}=\left\lceil\frac{n}{2}\right\rceil .
$$

Note que $h\left(K_{n}\right)=\left\lceil\frac{n}{2}\right\rceil$. Por outro lado, um grafo é desconexo se e somente se seu índice de expansão é zero. Note também que $h\left(K_{n}\right)$ atinge o limitante superior (3.1), e nesse sentido $K_{n}$ possui o índice 
de expansão ótimo. Porém, não consideramos grafos completos como expansores, dado que estamos interessados em grafos que possuem um número linear de arestas.

Para garantir a linearidade no número de arestas, definimos famílias de grafos expansores como famílias infinitas de grafos regulares de mesma valência cujos índices de expansão estão afastados de zero.

Definição 3.2 (Família de grafos expansores). Seja $d \in \mathbb{Z}_{++}$. Dizemos que uma sequência de grafos $d$-regulares $\left(G_{n}\right)_{n \in \mathbb{N}}$ é uma família de grafos expansores se $\left(\left|V\left(G_{n}\right)\right|\right)_{n \in \mathbb{N}}$ é crescente e se existe $\varepsilon>0$ tal que, para todo $n \in \mathbb{N}$, vale que $h\left(G_{n}\right) \geq \varepsilon$.

Note que todo grafo $G$ que está em uma família de grafos expansores $d$-regulares possui $d|V(G)| / 2$ arestas. Logo, grafos de uma família de expansores possuem um número linear de arestas. Além disso, os grafos dessas famílias são todos conexos e seus índices de expansão não convergem para zero.

Quando mencionamos um grafo expansor, estamos nos referindo a um grafo de tamanho assintótico que pertence a uma família de grafos expansores. Dessa forma, os grafos expansores são esparsos, conexos e seus índices de expansão estão afastados de zero. Mais adiante, mostraremos que existe um limitante superior justo para o índice de expansão de grafos expansores. Assim, consideramos como bons expansores os grafos expansores que possuem um índice de expansão próximo desse limitante.

Seria interessante dar um exemplo simples de famílias de grafos expansores, entretanto não se conhece nenhuma família de expansores que pode ser facilmente descrita. Por isso, com o objetivo de ilustrar o conceito de famílias de grafos expansores, mostraremos que a família de circuitos não é uma família de grafos expansores. Seja $\left(C_{n}\right)_{n \in \mathbb{N} \backslash\{0,1,2\}}$ uma família de grafos, onde $C_{n}$ é um circuito com $n$ vértices. Note que, para todo $n \in \mathbb{N} \backslash\{0,1,2\}$, vale que $h\left(C_{n}\right) \leq \frac{4}{n}$, o que implica que $\lim _{n \rightarrow \infty} h\left(C_{n}\right)=0$. Portanto, a família de circuitos não é uma família de grafos expansores.

Outro exemplo de famílias infinitas de grafos $d$-regulares que não são famílias expansoras é a família de grids. Seja $S:=\{(-1,0),(0,1),(0,-1),(1,0)\}$. Seja o grupo $H_{n}:=\left(\mathbb{Z}_{2 n} \times \mathbb{Z}_{2 n},+\right)$ para todo $n \in \mathbb{Z}_{++}$. Seja $\left(G_{n}\right)_{n \in \mathbb{Z}_{++}}$a família de grafos onde $G_{n}$ é um grid definido como $G_{n}:=$ $\operatorname{Cay}\left(H_{n}, S\right)$. Note que

$$
h\left(G_{n}\right) \leq \frac{\delta([n] \times[2 n])}{2 n^{2}}=\frac{4 n}{2 n^{2}}=\frac{2}{n}
$$

para todo $n \in \mathbb{Z}_{++}$. Logo, $\lim _{n \rightarrow \infty} h\left(G_{n}\right)=0$, e portanto $\left(G_{n}\right)_{n \in \mathbb{Z}_{++}}$não é uma família de grafos expansores.

\subsection{Caracterização espectral de grafos expansores}

Para saber se um grafo é um expansor, precisamos calcular o seu índice de expansão, e isso não é uma tarefa simples, dado que o problema de calcular o índice de expansão de um grafo é NP-difícil (vide [29]). Porém, existe uma forma de estimar o índice de expansão de um grafo regular utilizando apenas a diferença entre os dois maiores autovalores da sua matriz de adjacência. Essa estimativa dos índices de expansão se deve a Cheeger [7] e a Alon e Milman [1], que mostraram que o índice de expansão de um grafo $d$-regular $G$ é limitado superior e inferiormente por valores que dependem da diferença entre $d$ e o segundo maior autovalor da matriz de adjacência de $G$. Segundo essa estimativa, $h(G)$ é afastado de zero se e somente se $d-\lambda_{2}^{\downarrow}\left(A_{G}\right)$ também é afastado de zero.

Teorema 3.3 (Cheeger [7] e Alon e Milman [1]). Seja $G$ um grafo $d$-regular. Então

$$
\frac{d-\lambda_{2}^{\downarrow}\left(A_{G}\right)}{2} \leq h(G) \leq \sqrt{2 d\left(d-\lambda_{2}^{\downarrow}\left(A_{G}\right)\right)} .
$$


Note que, pelo Corolário 2.52, a diferença entre os dois maiores autovalores de um grafo $d$ regular $G$ é igual a $d-\lambda_{2}^{\downarrow}\left(A_{G}\right)$. Dessa forma, o índice de expansão de $G$ é afastado de zero se e somente se o gap espectral de $G$, que é diferença entre os dois maiores autovalores de $A_{G}$, também é afastado de zero. Pelo Teorema 3.3, podemos utilizar o gap espectral na definição de famílias de grafos expansores no lugar do índice de expansão, como mostrado a seguir.

Corolário 3.4. Seja $d \in \mathbb{Z}_{++}$. Seja $\left(G_{n}\right)_{n \in \mathbb{N}}$ uma sequência de grafos $d$-regulares tal que $\left(\left|V\left(G_{n}\right)\right|\right)_{n \in \mathbb{N}}$ é crescente. Então $\left(G_{n}\right)_{n \in \mathbb{N}}$ é uma família de grafos expansores se e somente se existe $\varepsilon>0$ tal que, para todo $n \in \mathbb{N}$, vale que

$$
d-\lambda_{2}^{\downarrow}\left(A_{G_{n}}\right) \geq \varepsilon .
$$

Demonstração. Suponha que $\left(G_{n}\right)_{n \in \mathbb{N}}$ é uma família de grafos expansores. Logo, existe $\varepsilon>0$ tal que $h\left(G_{n}\right) \geq \varepsilon$ para todo $n \in \mathbb{N}$. Pelo Teorema 3.3, para todo $n \in \mathbb{N}$, vale que

$$
\sqrt{2 d\left(d-\lambda_{2}^{\downarrow}\left(A_{G_{n}}\right)\right)} \geq h\left(G_{n}\right) \geq \varepsilon
$$

o que implica que

$$
d-\lambda_{2}^{\downarrow}\left(A_{G_{n}}\right) \geq \frac{\left(h\left(G_{n}\right)\right)^{2}}{2 d} \geq \frac{\varepsilon^{2}}{2 d} .
$$

Portanto, existe $\varepsilon^{\prime}>0$ tal que, para todo $n \in \mathbb{N}$, vale que $d-\lambda_{2}^{\downarrow}\left(A_{G_{n}}\right) \geq \varepsilon^{\prime}$.

Suponha agora que existe $\varepsilon>0$ tal que, para todo $n \in \mathbb{N}$, vale que $d-\lambda_{2}^{\downarrow}\left(A_{G_{n}}\right) \geq \varepsilon$. Note que, pelo Teorema 3.3, temos que

$$
h\left(G_{n}\right) \geq \frac{d-\lambda_{2}^{\downarrow}\left(A_{G_{n}}\right)}{2} \geq \frac{\varepsilon}{2} .
$$

Portanto, existe $\varepsilon^{\prime}>0$ tal que $h\left(G_{n}\right) \geq \varepsilon^{\prime}$ para todo $n \in \mathbb{N}$, o que implica que $\left(G_{n}\right)_{n \in \mathbb{N}}$ é uma família de grafos expansores.

Como existem algoritmos que calculam os autovalores de matrizes simétricas $n \times n$ em tempo $O\left(n^{3}\right)$, então é possível calcular os autovalores da matriz de adjacência de um grafo com $n$ vértices em tempo $O\left(n^{3}\right)$. Assim, calcular o índice de expansão de forma estimada pelo gap espectral traz algumas vantagens sobre calcular o índice de expansão exato, que é um problema NP-difícil. Além disso, a teoria espectral dos grafos é uma área abrangente, e por isso, além dos resultados envolvendo o índice de expansão, podemos obter resultados sobre expansores que envolvem o gap espectral de matrizes de adjacência. Algumas propriedades de grafos expansores obtidas através do gap espectral serão vistas na próxima seção.

\subsection{Propriedades expansoras}

Inicialmente, mostraremos que grafos expansores possuem alta condutibilidade nas arestas. Em outras palavras, é preciso remover um grande número de arestas para desconectar dois subconjuntos de vértices que possuem um grande número de arestas incidentes. A condutibilidade nas arestas é obtida através de um parâmetro de grafos chamado índice de condutibilidade, que é utilizado em algoritmos de clustering para verificar se determinado conjunto de pontos é um cluster, ou seja, se esse conjunto agrupa pontos semelhantes entre si, deixando de fora os pontos menos semelhantes (vide [20]). Algoritmos para clustering utilizam grafos completos com pesos nas arestas de forma que os vértices representam pontos, e o peso de cada aresta representa a semelhança entre suas pontas. Sejam $u, v_{1}, v_{2}$ vértices que representam pontos distintos. Se o ponto representado por $u$ 
possui maior semelhança com o ponto representado por $v_{1}$ do que com o ponto representado por $v_{2}$, então o peso da aresta $\left\{u, v_{1}\right\}$ é maior que o peso da aresta $\left\{u, v_{2}\right\}$. Como, neste capítulo, lidamos apenas com grafos sem pesos nas arestas, utilizamos arestas múltiplas para representar os pesos das arestas. Dizemos que um conjunto $S$ de vértices forma um cluster se $\delta(S)$ têm um número pequeno de arestas comparado ao número de arestas internas de $S$. Para verificar se um conjunto de pontos pode ser considerado como um cluster, utilizamos o índice de condutibilidade.

Definição 3.5. Seja $G=(V, E)$ um grafo conexo. O indice de condutibilidade de um subconjunto de vértices não vazio $S \subsetneq V$ é definido por

$$
\phi_{G}(S):=\frac{|\delta(S)|}{\min \{\operatorname{vol}(S), \operatorname{vol}(\bar{S})\}}
$$

onde $\operatorname{vol}(U)=\sum_{v \in U} \operatorname{grau}(v)$ para todo $U \subseteq V$. Defina também o índice de condutibilidade de $G$ como

$$
\phi(G):=\min _{\varnothing \neq S \subsetneq V} \phi_{G}(S) .
$$

Seja $G=(V, E)$ um grafo que representa um conjunto de pontos. Seja $\varnothing \neq S \subseteq V$. Suponha que $S$ forma um único cluster. Note que o número de arestas em $\delta_{G}(S)$ é pequeno comparado ao número de arestas que têm as duas pontas em $S$. Note também que todo corte em $G[S]$ tem tamanho grande comparado ao menor volume de uma margem. Logo, o índice de condutibilidade de todos os subconjuntos próprios e não vazios de um cluster é grande.

Suponha agora que $S$ seja a união disjunta de 2 clusters $S_{1}$ e $S_{2}$. Note que o número de arestas com duas pontas em $S_{1}$ e com duas pontas em $S_{2}$ é grande comparado ao número de arestas de $\delta_{G[S]}\left(S_{1}\right)$ e $\delta_{G[S]}\left(S_{2}\right)$. Assim, os índices de condutibilidade de $S_{1}$ e de $S_{2}$ são pequenos. Logo, o índice de condutibilidade de $G[S]$ é pequeno. Suponha que $S$ é um subconjunto próprio de um conjunto que forma um único cluster. Note que o número de arestas em $\delta_{G}(S)$ é grande comparado ao número de arestas com duas pontas em $S$. Dessa forma, o volume de pelo menos um subconjunto não vazio de $S$ é grande comparado ao número de arestas do corte desse subconjunto. Logo, o índice de condutibilidade de $G[S]$ é pequeno. Portanto, se um grafo possui índice de condutibilidade grande, então esse grafo forma um único cluster.

Seja $G=(V, E)$ um grafo conexo $d$-regular. Note que

$$
\phi_{G}(S)=\frac{|\delta(S)|}{\min \{|S|,|\bar{S}|\} d} \geq \frac{h(G)}{d}
$$

para todo $S \subsetneq V$ não vazio. Logo, se $h(G)$ está afastado de zero, então o índice de condutibilidade de todo subconjunto não vazio $S \subsetneq V$ também está afastado de zero, o que implica que $\phi(G)$ está afastado de zero. Assim, como grafos expansores possuem um alto índice de expansão, então eles também possuem um alto índice de condutibilidade. Logo, podemos dizer que expansores formam um único cluster.

Essa propriedade de expansores é a única apresentada nesta seção que não usa a caracterização de Cheeger e de Alon e Milman, utilizando como limitante o próprio índice de expansão.

A seguir, mostraremos duas propriedades que são consideradas propriedades de grafos expansores por utilizarem valores que dependem do segundo maior autovalor como limitantes. Porém, nem todos os grafos expansores possuem essas duas propriedades, dado que na verdade elas utilizam como limitantes valores que dependem de um parâmetro de grafos definido como $\lambda(G):=\max \left\{\left|\lambda_{2}^{\downarrow}\left(A_{G}\right)\right|,\left|\lambda_{\min }\left(A_{G}\right)\right|\right\}$ para todo grafo $G$. Assim, além de dependerem do segundo 
maior autovalor, essas propriedades também dependem do menor autovalor da matriz de adjacência do grafo.

Uma dessas propriedades é baseada no Expander Mixing Lemma. Uma das consequências desse resultado é que, se $G=(V, E)$ é um grafo $d$-regular com $\lambda(G)$ afastado de $d$, então o número de arestas em qualquer corte de $G$ se concentra em torno do número esperado de arestas no respectivo corte de um grafo aleatório que possui $|V|$ vértices e cujo número esperado de arestas é $|E|$. Desta forma, podemos dizer que grafos $d$-regulares com $\lambda$ afastado de $d$ se comportam como grafos aleatórios, e por esse motivo, são chamados de grafos pseudo-aleatórios. Por exemplo, um grafo pseudo-aleatório $d$-regular $G$ com $d-\lambda(G) \geq 2$ é $d$-aresta-conexo, ou seja, é necessário remover pelo menos $d$ arestas para desconectá-lo. Além disso, se $G$ possui um número par de vértices, então ele possui pelo menos um emparelhamento perfeito (vide [21, Teorema 4.3]).

Lema 3.6 (Expander Mixing Lemma). Seja $G$ um grafo $d$-regular com $n$ vértices, com $d \geq 1$ e $n \geq 2$. Então, para todo $S, T \subseteq V(G)$, vale que

$$
|| E(S, T)\left|-\frac{d|S||T|}{n}\right| \leq \lambda(G) \sqrt{|S||T|}
$$

Demonstração. Defina $A:=A_{G}$ e $\lambda_{i}:=\lambda_{i}^{\downarrow}(A)$ para todo $i \in[n]$. Sejam $u_{1}, u_{2}, \ldots, u_{n}$ autovetores ortonormais de $A$ associados aos autovalores $\lambda_{1}, \lambda_{2}, \ldots, \lambda_{n}$, respectivamente. Pelo Teorema 2.39, temos que

$$
A=\sum_{i \in[n]} \lambda_{i} u_{i} u_{i}^{\top}
$$

Pelo Corolário 2.52, temos que $\lambda_{1}=d$ e $u_{1}=\frac{1}{\sqrt{n}} \mathbb{1}$.

Sejam $S, T \subseteq V(G)$. Como $u_{1}, u_{2}, \ldots, u_{n}$ formam uma base ortonormal de $A$, então existem $\alpha, \beta \in \mathbb{R}^{n}$ tais que $\mathbb{1}_{S}=\sum_{i \in[n]} \alpha_{i} u_{i}$ e $\mathbb{1}_{T}=\sum_{i \in[n]} \beta_{i} u_{i}$. Note que, para todo $i \in[n]$, vale que

$$
\begin{aligned}
& u_{i}^{\top} \mathbb{1}_{S}=u_{i}^{\top} \sum_{j \in[n]} \alpha_{j} u_{j}=\alpha_{i} u_{i}^{\top} u_{i}=\alpha_{i}, \\
& u_{i}^{\top} \mathbb{1}_{T}=u_{i}^{\top} \sum_{j \in[n]} \beta_{j} u_{j}=\beta_{j} u_{i}^{\top} u_{i}=\beta_{j} .
\end{aligned}
$$

Note também que

$$
\begin{aligned}
|E(S, T)| & =\mathbb{1}_{S}^{\top} A \mathbb{1}_{T}=\left(\sum_{i \in[n]} \alpha_{i} u_{i}\right)^{\top} A\left(\sum_{i \in[n]} \beta_{i} u_{i}\right) \\
& =\left(\sum_{i \in[n]} \alpha_{i} u_{i}\right)^{\top}\left(\sum_{i \in[n]} \beta_{i} A u_{i}\right) \\
& =\left(\sum_{i \in[n]} \alpha_{i} u_{i}\right)^{\top}\left(\sum_{i \in[n]} \beta_{i} \lambda_{i} u_{i}\right) \\
& =\sum_{i \in[n]} \alpha_{i} \beta_{i} \lambda_{i} \\
& =\alpha_{1} \beta_{1} \lambda_{1}+\sum_{i=2}^{n} \alpha_{i} \beta_{i} \lambda_{i} \\
& =d \frac{1}{n} \mathbb{1}^{\top} \mathbb{1}_{S} \mathbb{1}^{\top} \mathbb{1}_{T}+\sum_{i=2}^{n} \alpha_{i} \beta_{i} \lambda_{i}
\end{aligned}
$$




$$
=\frac{d|S||T|}{n}+\sum_{i=2}^{n} \alpha_{i} \beta_{i} \lambda_{i} .
$$

Logo, vale que

$$
\begin{aligned}
|| E(S, T)\left|-\frac{d|S||T|}{n}\right| & =\left|\sum_{i \in\{2, \ldots, n\}} \alpha_{i} \beta_{i} \lambda_{i}\right| \leq \sum_{i \in\{2, \ldots, n\}}\left|\alpha_{i} \beta_{i} \lambda_{i}\right| \\
& \leq \lambda(G) \sum_{i \in\{2, \ldots, n\}}\left|\alpha_{i} \beta_{i}\right| \leq \lambda(G) \sum_{i \in[n]}\left|\alpha_{i} \beta_{i}\right| \\
& \leq \lambda(G)\|\alpha\|_{2}\|\beta\|_{2},
\end{aligned}
$$

onde a última desigualdade vale pelo Lema 2.16.

Por (3.2) e (3.3), temos que

$$
\begin{aligned}
& \|\alpha\|_{2}^{2}=\sum_{i \in[n]} \alpha_{i}^{2}=\sum_{i \in[n]}\left(u_{i}^{\top} \mathbb{1}_{S}\right)^{2}=\sum_{i \in[n]} \mathbb{1}_{S}^{\top} u_{i} u_{i}^{\top} \mathbb{1}_{S}=\mathbb{1}_{S}^{\top} \mathbb{1}_{S}=\left\|\mathbb{1}_{S}\right\|_{2}^{2}=|S|, \\
& \|\beta\|_{2}^{2}=\sum_{i \in[n]} \beta_{i}^{2}=\sum_{i \in[n]}\left(u_{i}^{\top} \mathbb{1}_{T}\right)^{2}=\sum_{i \in[n]} \mathbb{1}_{T}^{\top} u_{i} u_{i}^{\top} \mathbb{1}_{T}=\mathbb{1}_{T}^{\top} \mathbb{1}_{T}=\left\|\mathbb{1}_{T}\right\|_{2}^{2}=|T| .
\end{aligned}
$$

Portanto, o resultado segue de (3.4) e (3.5).

Sejam $d, n \in \mathbb{N} \backslash\{0,1\}$ e $p:=d /(n-1)$. Seja $G(n, p)$ um grafo aleatório no modelo de ErdôsRényi, isto é, o conjunto de vértices é $[n]$ e cada possível aresta é sorteada independentemente com probabilidade $p$. Note que, para quaisquer $S, T \subseteq[n]$ disjuntos, o número esperado de arestas entre $S$ e $T$ em $G(n, p)$ é $p|S||T|$. Note também que grafos aleatórios $G(n, p)$ e um grafo $d$-regular com $n$ vértices possuem a mesma densidade, que é a proporção entre o número de arestas do grafo e o número de arestas de um grafo completo com o mesmo número de vértices. Portanto, se $G$ é um grafo $d$-regular com $n$ vértices e com $\lambda(G)$ afastado de $d$, então o número de arestas entre quaisquer subconjuntos $S, T \subseteq V(G)$ disjuntos se concentra em torno do número de arestas esperado entre quaisquer subconjuntos de tamanho $|S|$ e $|T|$ de grafos aleatórios $G(n, p)$, que possuem o mesmo tamanho e a mesma densidade de $G$. Assim, podemos dizer que os grafos expansores se comportam como grafos aleatórios que possuem o mesmo número de vértices e o número esperado de arestas igual ao número de arestas do expansor em questão.

A terceira propriedade de expansores que iremos descrever segue do Teorema 3.7, que diz que, se $G$ é um grafo $d$-regular com $\lambda(G)$ afastado de $d$, então a distribuição de probabilidade do último vértice de um passeio aleatório desse grafo converge rapidamente para a distribuição uniforme.

Teorema 3.7. Sejam $G$ um grafo $d$-regular com $n$ vértices e $p$ um vetor de probabilidade. Então,

$$
\left\|\left(\frac{A_{G}}{d}\right)^{t} p-\frac{1}{n} \mathbb{1}\right\|_{2} \leq\left(\frac{\lambda(G)}{d}\right)^{t}
$$

para todo $t \in \mathbb{Z}_{++}$.

Demonstração. Tome $u:=\frac{1}{n} \mathbb{1}, A:=\frac{1}{d} A_{G}$ e $\alpha:=\lambda(G) / d$. Pelo Corolário 2.52, temos que $A u=u$. Assim, para todo $t \in \mathbb{Z}_{++}$,

$$
\left\|A^{t} p-u\right\|_{2}^{2}=\left\|A^{t} p-A^{t} u\right\|_{2}^{2}=\left\|A^{t}(p-u)\right\|_{2}^{2}
$$




$$
\begin{aligned}
& =(p-u)^{\top}\left(A^{t}\right)^{\top} A^{t}(p-u) \\
& \leq\left(\alpha^{t}\right)^{2}(p-u)^{\top}(p-u),
\end{aligned}
$$

onde a desigualdade segue do Lema 2.30, pois $p-u$ é ortogonal a $u$, e $u$ é um autovetor de $A$ associado a $\lambda_{\max }(A)$. Note também que

$$
(p-u)^{\top}(p-u)=(p-u)^{\top} p \leq p^{\top} p \leq \mathbb{1}^{\top} p=1 .
$$

Portanto, $\left\|A^{t} p-u\right\|_{2} \leq \alpha^{t}$ para todo $t \in \mathbb{Z}_{++}$.

Sejam $G$ um grafo e $t \in \mathbb{Z}_{++}$. Para todo $u, v \in V(G)$, a probabilidade de um passeio aleatório de comprimento $t$ que começa em $u$ terminar em $v$ é $\left(\frac{1}{d} A_{G}\right)^{t}(u, v)$. Então $\left(\frac{1}{d} A_{G}\right)^{t} p$ é a distribuição de probabilidade do último vértice de um passeio aleatório, cujo primeiro vértice é escolhido de acordo com o vetor de probabilidade $p$.

Pelo Lema 2.51, temos que $\lambda(G) / d \leq 1$ para todo grafo $d$-regular $G$. Logo, pelo Teorema 3.7, em um grafo $d$-regular $G=(V, E)$ com $\lambda(G)$ afastado de $d$, não é necessário um $t$ grande para que $\left\|\left(\frac{A_{G}}{d}\right)^{t} p-\frac{1}{|V|} \mathbb{1}\right\|_{2}$ seja próximo de zero. Assim, se considerarmos um grafo expansor, a distribuição de probabilidade do último vértice de um passeio aleatório converge rapidamente para a distribuição uniforme.

Seja $G$ um grafo $d$-regular conexo e não bipartido que não possui necessariamente $\lambda(G)$ afastado de $d$. Pelos Lemas 2.51 e 2.53, temos que $\lambda(G) / d<1$, o que implica que a convergência da sequência

$$
\lim _{t \rightarrow \infty}\left\|\left(\frac{A_{G}}{d}\right)^{t} p-\frac{1}{n} \mathbb{1}\right\|_{2} \leq \lim _{t \rightarrow \infty}\left(\frac{\lambda(G)}{d}\right)^{t}=0
$$

vale mesmo que $\lambda(G)$ não esteja afastado de $d$. Portanto, a distribuição de probabilidade do último vértice de um passeio aleatório em um grafo $d$-regular conexo e não bipartido converge para a distribuição uniforme mesmo que esse grafo não seja expansor. O que os diferencia é que um grafo expansor não precisa de um número grande de passos para convergir para uma distribuição uniforme devido ao seu $\lambda$ afastado de $d$.

Note que as duas últimas propriedades são características de um subconjunto próprio de grafos expansores, já que uma das condições dessas propriedades (referentes ao Lema 3.6 e ao Teorema 3.7) é que o valor absoluto do menor autovalor da matriz de adjacência do grafo também seja pequeno. Ou seja, as duas últimas propriedades mostradas anteriormente valem para um subconjunto de grafos expansores, e não para expansores em geral.

Por exemplo, se considerarmos um grafo expansor bipartido $d$-regular $G$, então, pelo Teorema 2.50, temos que $\lambda(G)=d$. Assim, não temos garantia de que o número de arestas entre quaisquer subconjuntos disjuntos $S, T \subseteq V(G)$ se concentra tão próximo, comparado a expansores com $\lambda$ menor, do número esperado de arestas entre quaisquer subconjuntos de tamanho $|S|$ e $|T|$ de grafos aleatórios com o mesmo tamanho e a mesma densidade de $G$. Além disso, os passeios aleatórios de $G$ não convergem para a distribuição uniforme.

\subsection{Grafos de Ramanujan}

Definimos famílias de grafos expansores e também mostramos algumas de suas propriedades que valem justamente pelo índice de expansão dos grafos dessas famílias serem afastados de zero. Mas, 
até o momento, não sabemos o que são expansores ótimos, pois não apresentamos um limitante superior para os índices de expansão e nem para o gap espectral de famílias de grafos expansores.

Com o intuito de encontrar um limitante para o gap espectral dos grafos de famílias de expansores, utilizamos um resultado assintótico de Alon e Boppana (vide [27]), que mostra que o segundo maior autovalor da matriz de adjacência de um grafo regular é limitado inferiormente por um valor que depende da sua valência.

Lema 3.8. Seja $G=(V, E)$ um grafo com $n$ vértices e com $\Delta(G)>1$. Seja $v \in V$. Então existe $u \in V$ tal que $\operatorname{dist}_{G}(u, v) \geq \log _{\Delta(G)}(n)$.

Demonstração. Como cada vértice tem grau no máximo $\Delta(G)$, então

$$
\left|\left\{u \in V: \operatorname{dist}_{G}(u, v)<k\right\}\right| \leq \Delta(G)^{k}-1
$$

para todo $k \in \mathbb{N} \backslash\{0\}$. Assim, vale que

$$
\left|\left\{u \in V: \operatorname{dist}_{G}(u, v)<\log _{\Delta(G)}(n)\right\}\right| \leq n-1 .
$$

Portanto, existe $u \in V$ tal que $\operatorname{dist}_{G}(u, v) \geq \log _{\Delta(G)}(n)$.

Definição 3.9. Seja $G=(V, E)$ um grafo. Denote $\operatorname{dist}_{G}(u, e):=\min \left\{\operatorname{dist}_{G}(u, v): v \in e\right\}$ para todo $u \in V$ e todo $e \in E$. Denote também $\operatorname{dist}_{G}(e, \bar{e}):=\min \{\operatorname{dist}(u, e): u \in \bar{e}\}$ para todos $e, \bar{e} \in E$.

Teorema 3.10 (Alon-Boppana, Nilli [27]). Seja $G=(V, E)$ um grafo $d$-regular com $d \geq 2$. Seja $c$ o comprimento máximo de um caminho mínimo de $G$. Então

$$
\lambda_{2}^{\downarrow}\left(A_{G}\right) \geq 2 \sqrt{d-1}-\frac{2 \sqrt{d-1}-1}{\frac{c}{2}-1} .
$$

Demonstração. Seja $n:=|V|$. Defina $k:=\frac{c}{2}-2$. Note que existem $e_{u}, e_{v} \in E$ tais que

$$
\operatorname{dist}_{G}\left(e_{u}, e_{v}\right) \geq c-2=2 k+2 .
$$

Sejam $u_{1}, u_{2}$ as pontas de $e_{u}$ e $v_{1}, v_{2}$ as pontas de $e_{v}$. Sejam

$$
U_{i}:=\left\{u \in V: \operatorname{dist}_{G}\left(u, e_{u}\right)=i\right\} \quad \text { e } \quad V_{i}:=\left\{v \in V: \operatorname{dist}_{G}\left(v, e_{v}\right)=i\right\}
$$

para todo $i \in\{0,1, \ldots, k\}$, como mostrado na Figura 3.1. Tome $U^{\prime}:=\bigcup_{i=0}^{k} U_{i}$ e $V^{\prime}:=\bigcup_{i=0}^{k} V_{i}$.

Como $\operatorname{dist}_{G}\left(e_{u}, e_{v}\right) \geq 2 k+2$, então $U_{0}, \ldots, U_{k}, V_{0}, \ldots, V_{k}$ são dois a dois disjuntos e

$$
\left|e \cap U^{\prime}\right| \neq 1 \text { ou }\left|e \cap V^{\prime}\right| \neq 1 \quad \forall e \in E .
$$

Além disso, para todo $i \in[k]$, vale que

$$
\left|U_{i}\right| \leq(d-1)\left|U_{i-1}\right| \quad \text { e } \quad\left|V_{i}\right| \leq(d-1)\left|V_{i-1}\right| .
$$

Sejam $a \in \mathbb{R}_{++}$e $b \in \mathbb{R}_{--}$tais que

$$
\sum_{i=0}^{k}\left(a\left|U_{i}\right|(d-1)^{-\frac{i}{2}}+b\left|V_{i}\right|(d-1)^{-\frac{i}{2}}\right)=0 .
$$




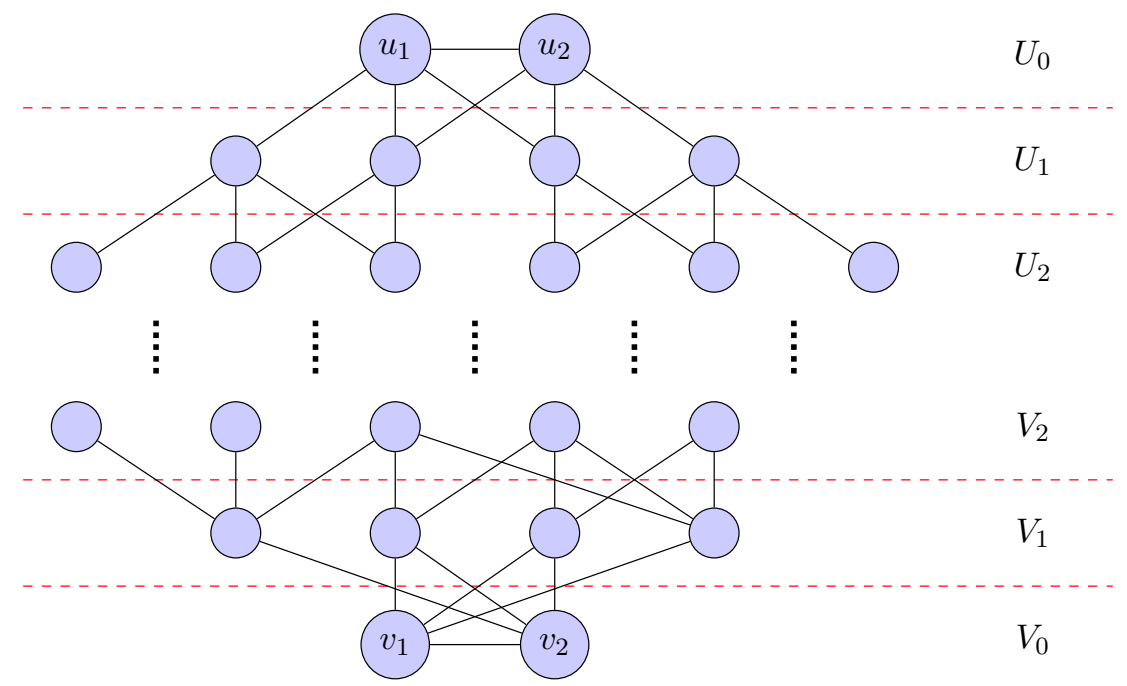

Figura 3.1: Representação dos conjuntos $U_{0}, U_{1}, \ldots, U_{k}$ e $V_{0}, V_{1}, \ldots, V_{k}$.

Defina $f: V \rightarrow \mathbb{R}$ como

$$
f(v):= \begin{cases}a(d-1)^{-\frac{i}{2}}, & \text { se } v \in U_{i} \\ b(d-1)^{-\frac{i}{2}}, & \text { se } v \in V_{i} \\ 0, & \text { se } v \in V \backslash\left(U^{\prime} \cup V^{\prime}\right)\end{cases}
$$

para todo $i \in\{0, \ldots, k\}$. Por (3.8), temos que $\mathbb{1}^{\top} f=0$. Logo, pelo Lema 2.30, temos que

$$
\lambda_{2}^{\downarrow}\left(A_{G}\right) \geq \frac{f^{\top} A_{G} f}{f^{\top} f} .
$$

Assim, como $G$ é $d$-regular, então

$$
\begin{aligned}
\lambda_{2}^{\downarrow}\left(A_{G}\right) f^{\top} f & \geq f^{\top} A_{G} f=\sum_{\{u, v\} \in E} 2 f(u) f(v) \\
& =\sum_{\{u, v\} \in E}\left(f(u)^{2}+f(v)^{2}\right)-\sum_{\{u, v\} \in E}(f(u)-f(v))^{2} \\
& =d \sum_{u \in V} f(u)^{2}-\sum_{\{u, v\} \in E}(f(u)-f(v))^{2} \\
& =d f^{\top} f-\sum_{\{u, v\} \in E}(f(u)-f(v))^{2} .
\end{aligned}
$$

Tome

$$
A:=a^{2} \sum_{i=0}^{k} \frac{\left|U_{i}\right|}{(d-1)^{i}} \quad \text { e } \quad B:=b^{2} \sum_{i=0}^{k} \frac{\left|V_{i}\right|}{(d-1)^{i}} .
$$

Note que $f^{\top} f=A+B$. Denote $E_{U}:=E\left[U^{\prime}\right] \cup \delta_{G}\left(U^{\prime}\right)$ e $E_{V}:=E\left[V^{\prime}\right] \cup \delta_{G}\left(V^{\prime}\right)$. Note que, para todo $i \in\{0, \ldots, k\}$ e cada $u, v \in U_{i}$, vale que $f(u)-f(v)=0$. Logo, vale que

$$
\sum_{\{u, v\} \in E_{U}}(f(u)-f(v))^{2}=\sum_{\{u, v\} \in E\left[U^{\prime}\right]}(f(u)-f(v))^{2}+\sum_{\{u, v\} \in \delta_{G}\left(U^{\prime}\right)}(f(u)-f(v))^{2}
$$




$$
\begin{aligned}
& \leq \sum_{\{u, v\} \in E\left[U^{\prime}\right]}(f(u)-f(v))^{2}+a^{2}\left|U_{k}\right| \frac{d-1}{(d-1)^{k}} \\
& \leq a^{2} \sum_{i=0}^{k-1}\left|U_{i}\right|(d-1)\left(\frac{1}{(d-1)^{\frac{i}{2}}}-\frac{1}{(d-1)^{\frac{i+1}{2}}}\right)^{2}+a^{2}\left|U_{k}\right| \frac{d-1}{(d-1)^{k}} \\
& =a^{2} \sum_{i=0}^{k-1}\left|U_{i}\right| \frac{1}{(d-1)^{i}}((d-1)+1-2 \sqrt{d-1})+a^{2}\left|U_{k}\right| \frac{d-1}{(d-1)^{k}} \\
& =A(d-2 \sqrt{d-1})-a^{2}\left|U_{k}\right| \frac{d-2 \sqrt{d-1}}{(d-1)^{k}}+a^{2}\left|U_{k}\right| \frac{d-1}{(d-1)^{k}} \\
& =A(d-2 \sqrt{d-1}))+\frac{a^{2}\left|U_{k}\right|}{(d-1)^{k}}(2 \sqrt{d-1}-1) .
\end{aligned}
$$

Por (3.7), temos que

$$
\frac{\left|U_{k}\right|}{(d-1)^{k}} \leq \frac{\left|U_{k-1}\right|}{(d-1)^{k-1}} \leq \cdots \leq \frac{\left|U_{0}\right|}{(d-1)^{0}}
$$

o que implica que

$$
a^{2} \frac{\left|U_{k}\right|}{(d-1)^{k}} \leq \frac{A}{k+1}
$$

Portanto,

$$
\sum_{\{u, v\} \in E_{U}}(f(u)-f(v))^{2} \leq A\left(d-2 \sqrt{d-1}+\frac{2 \sqrt{d-1}-1}{k+1}\right) .
$$

De forma análoga, obtemos que

$$
\sum_{\{u, v\} \in E_{V}}(f(u)-f(v))^{2} \leq B\left(d-2 \sqrt{d-1}+\frac{2 \sqrt{d-1}-1}{k+1}\right) .
$$

Logo, vale que

$$
\begin{aligned}
\sum_{\{u, v\} \in E}(f(u)-f(v))^{2} & =\sum_{\{u, v\} \in E_{U}}(f(u)-f(v))^{2}+\sum_{\{u, v\} \in E_{V}}(f(u)-f(v))^{2} \\
& \leq(A+B)\left(d-2 \sqrt{d-1}+\frac{2 \sqrt{d-1}-1}{k+1}\right) .
\end{aligned}
$$

Portanto, por (3.9), temos que

$$
\begin{aligned}
\lambda_{2}^{\downarrow}\left(A_{G}\right) & \geq d-\sum_{\{u, v\} \in E} \frac{(f(u)-f(v))^{2}}{f^{\top} f} \geq 2 \sqrt{d-1}-\frac{2 \sqrt{d-1}-1}{k+1} \\
& =2 \sqrt{d-1}-\frac{2 \sqrt{d-1}-1}{\frac{c}{2}-1} .
\end{aligned}
$$

Teorema 3.11. Sejam $\varepsilon>0$ e $d \in \mathbb{Z} \operatorname{com} d \geq 2$. Então, existe $N \in \mathbb{N}$ tal que, para todo $n \in \mathbb{N}$ com $n \geq N$ e para todo grafo $d$-regular $G$ com $n$ vértices, vale que

$$
\lambda_{2}^{\downarrow}\left(A_{G}\right) \geq 2 \sqrt{d-1}-\varepsilon .
$$


Demonstração. Sejam $\alpha:=\frac{4 \sqrt{d-1}-2}{\varepsilon}+2$ e $N:=\left\lceil d^{\alpha}\right\rceil$. Pelo Lema 3.8, temos que, para todo grafo $d$-regular $G$ com $n \geq N$ vértices, o comprimento máximo de um caminho mínimo de $G$ é pelo menos $\log _{d}(n)$. Portanto, pelo Teorema 3.10, temos que

$$
\begin{aligned}
\lambda_{2}^{\downarrow}\left(A_{G}\right) & \geq 2 \sqrt{d-1}-\frac{2 \sqrt{d-1}-1}{\frac{\log _{d}(n)}{2}-1} \geq 2 \sqrt{d-1}-\frac{2 \sqrt{d-1}-1}{\frac{\log _{d}(N)}{2}-1} \\
& \geq 2 \sqrt{d-1}-\frac{2 \sqrt{d-1}-1}{\frac{\alpha}{2}-1}=2 \sqrt{d-1}-\frac{2 \sqrt{d-1}-1}{\frac{2 \sqrt{d-1}-1}{\varepsilon}} \\
& =2 \sqrt{d-1}-\varepsilon .
\end{aligned}
$$

Com esse resultado, podemos considerar como famílias expansoras ótimas, caso existam, as famílias de grafos expansores $d$-regulares $\left(G_{i}\right)_{i \in \mathbb{N}}$, com $d>2$, tais que $\lambda_{2}^{\downarrow}\left(A_{G_{i}}\right) \leq 2 \sqrt{d-1}$ para todo $i \in \mathbb{N}$. A existência dessas famílias foi provada em 1988 por Lubotzky, Phillips e Sarnak [23] e Margulis [26] para valências específicas, e em 2013 por Marcus, Spielman e Srivastava [24] para todas as valências. Logo, consideramos como expansores ótimos os grafos $d$-regulares, com $d>2$, de tamanho assintótico cujo segundo maior autovalor de sua matriz de adjacência é limitado superiormente por $2 \sqrt{d-1}$. Isso motiva a definição de grafos de Ramanujan, que são grafos $d$-regulares com $d>2$ tal que o valor absoluto dos autovalores não-triviais (autovalores cujos valores absolutos diferem de $d$ ) de sua matriz de adjacência é no máximo $2 \sqrt{d-1}$.

Definição 3.12 (Grafos de Ramanujan). Seja $G=(V, E)$ um grafo $d$-regular conexo com $n$ vértices, e com $d>2$. Dizemos que $G$ é um grafo de Ramanujan se, para todo $i \in[n]$, vale que

$$
\left|\lambda_{i}^{\downarrow}\left(A_{G}\right)\right| \leq 2 \sqrt{d-1} \quad \text { ou } \quad\left|\lambda_{i}^{\downarrow}\left(A_{G}\right)\right|=d .
$$

Pelas Proposições 2.54 e 2.55, é fácil ver que grafos completos e grafos bipartidos completos de valências maiores que 2 são exemplos de grafos de Ramanujan. Entretanto, grafos completos não são expansores desejáveis, pois não é possível criar uma família infinita de grafos completos de mesma valência, e o mesmo vale para grafos bipartidos completos.

Note que mencionamos anteriormente que um expansor ótimo deve ter valência maior que 2 e também definimos os grafos de Ramanujan apenas com valências maiores que 2. Mostraremos agora o motivo pelo qual essa restrição da valência é necessária. Seja $\left(G_{n}\right)_{n \in \mathbb{N}}$ uma família de grafos de Ramanujan $d$-regulares. Logo, vale que $d>2$. Então, pelo Teorema 3.3, temos que

$$
2 h\left(G_{n}\right) \geq d-\lambda_{2}^{\downarrow}\left(A_{G_{n}}\right) \geq d-2 \sqrt{d-1} \geq 3-2 \sqrt{2}>0
$$

para todo $n \in \mathbb{N}$. Portanto, famílias infinitas de grafos de Ramanujan de valência fixa maior que 2 são famílias de grafos expansores.

Suponha que $\left(G_{n}\right)_{n \in \mathbb{N}}$ é uma família de grafos conexos 2-regulares. Note que $\left(G_{n}\right)_{n \in \mathbb{N}}$ é uma família de circuitos e, como mostrado anteriormente, não é uma família de grafos expansores. Além disso, pelo Teorema 3.11, temos que, para todo $\varepsilon>0$, existe $n \in \mathbb{N}$ tal que

$$
\frac{d-\lambda_{2}^{\downarrow}\left(A_{G_{n}}\right)}{2} \leq \frac{d-2 \sqrt{d-1}+\varepsilon}{2}=\frac{\varepsilon}{2}
$$

para $d=2$ e todo $n \in \mathbb{N}$. Portanto, o Teorema 3.3 não garante que os índices de expansão de todos os grafos da família sejam maiores que um único valor positivo. Assim, como os grafos de Ramanujan foram definidos como expansores ótimos, então sua definição se restringe a valências maiores que 2 . 


\subsection{Expansores irregulares}

Os grafos expansores definidos anteriormente são regulares, porém utilizamos outra definição de expansores que também engloba grafos irregulares. Dizemos que um grafo é expansor se o segundo maior autovalor de sua matriz de adjacência está relativamente afastado do maior autovalor de sua matriz de adjacência. Mostraremos mais adiante que algumas propriedades de grafos expansores também se aplicam a esses grafos.

Para mostrar quem são as famílias expansoras irregulares ótimas, utilizamos o resultado abaixo, que é uma variação do resultado de Alon e Boppana, mostrado no Teorema 3.11.

Teorema 3.13 (Greenberg [13]). Sejam $\varepsilon>0$ e $G^{\prime}$ um grafo. Então, existe $N \in \mathbb{N}$ tal que para todo $n \in \mathbb{N}$ com $n \geq N$ e para todo grafo conexo $G$ bipartido com $n$ vértices tal que $T_{G}=T_{G^{\prime}}$, vale que

$$
\lambda_{2}^{\downarrow}\left(A_{G}\right) \geq \rho\left(T_{G^{\prime}}\right)-\varepsilon .
$$

Dessa forma, podemos considerar como famílias expansoras irregulares ótimas, caso existam, as famílias de expansores $\left(G_{i}\right)_{i \in \mathbb{N}}$, com $T_{G_{i}}=T_{G_{j}}$ para todos $i, j \in \mathbb{N}$, tais que $\lambda_{2}^{\downarrow}\left(A_{G_{i}}\right) \leq \rho\left(T_{G_{i}}\right)$ para todo $i \in \mathbb{N}$. A prova de existência dessas famílias se deve a Marcus, Spielman e Srivastava [24] e será mostrada mais adiante. 


\section{Capítulo 4}

\section{Árvores entrelaçadoras de polinômios}

Neste capítulo, definiremos entrelaçadores comuns, que são polinômios cujas raízes entrelaçam as raízes de todos os polinômios de um determinado conjunto. Conjuntos de polinômios com coeficientes dominantes positivos que têm um entrelaçador comum possuem a seguinte propriedade: a raiz máxima de algum polinômio do conjunto é limitada superiormente pela raiz máxima da soma de todos os polinômios do conjunto. Essa propriedade de raízes máximas seria útil para provar alguns resultados para certos conjuntos de polinômios que não possuem necessariamente entrelaçadores comuns, mas que podem ser particionados de forma que cada parte possui um entrelaçador comum, entre outras propriedades. Exemplos desses resultados são a prova de existência de grafos expansores ótimos e a prova de uma solução do Problema de Kadison-Singer. Com o objetivo de utilizar a propriedade de raízes máximas nesses resultados, definimos as árvores entrelaçadoras de polinômios, que são árvores cujos nós representam polinômios de coeficientes dominantes positivos, de forma que todo conjunto de polinômios representados pelos filhos de um mesmo nó possui um entrelaçador comum. Os polinômios representados pelas folhas dessas árvores, apesar de não possuírem necessariamente um único entrelaçador comum, possuem a propriedade de raízes máximas citada anteriormente. A razão para utilizar as árvores entrelaçadoras de polinômios neste texto é que os polinômios usados nas provas de existência de bons expansores e na prova de uma solução do Problema de Kadison-Singer formam árvores entrelaçadoras de polinômios. Assim, esses polinômios possuem a propriedade de raízes máximas mesmo não tendo necessariamente um entrelaçador comum.

\subsection{Entrelaçamento de polinômios}

A definição de entrelaçamento de polinômios é simples. Dizemos que dois polinômios se entrelaçam se suas raízes se entrelaçam.

Definição 4.1. Sejam $p, q \in \mathbb{R}[x]$ polinômios de graus $d-1$ e $d \geq 2$, respectivamente, que possuem somente raízes reais. Dizemos que $p$ entrelaça $q$ se

$$
r_{1}(q) \leq r_{1}(p) \leq r_{2}(q) \leq r_{2}(p) \leq \cdots \leq r_{d-1}(p) \leq r_{d}(q)
$$

Como exemplo, temos os polinômios $p, q \in \mathbb{R}[x]$ definidos por

$$
p(x):=x^{2}-9 x+18 \text { e } q(x):=x^{3}-11 x^{2}+34 x-24 .
$$

Note que esses polinômios podem ser fatorados como

$$
p(x)=(x-3)(x-6) \quad \text { e } \quad q(x)=(x-1)(x-4)(x-6) .
$$


Como as raízes de $p$ entrelaçam as raízes de $q$, então $p$ entrelaça $q$. Vale também que o polinômio $x^{3}$ entrelaça o polinômio $x^{4}$, pois ambos possuem todas as raízes iguais a zero.

Outros exemplos de polinômios que se entrelaçam são um polinômio $p$ de grau pelo menos $2 \mathrm{e}$ o polinômio que resulta da derivada de $p$, como mostrado a seguir.

Proposição 4.2. Seja $p \in \mathbb{R}[x]$ um polinômio de grau $d \geq 2$ que possui somente raízes reais. Então $p^{\prime}$ entrelaça $p$.

Demonstração. Sejam $i, j \in[d]$ com $i \leq j$ tais que $r_{i}(p)=r_{j}(p)=: \alpha$. Assim, existe um polinômio $\bar{p} \in \mathbb{R}[x]$ tal que $p=(x-\alpha)^{j-i+1} \bar{p}$. Logo, vale que

$$
p^{\prime}=(x-\alpha)^{j-i+1} \bar{p}^{\prime}+(j-i+1)(x-\alpha)^{j-i} \bar{p}=(x-\alpha)^{j-i}\left((x-\alpha) \bar{p}^{\prime}+(j-i+1) \bar{p}\right),
$$

o que implica que $\alpha$ é raiz de $p^{\prime}$ com multiplicidade pelo menos $j-i$. Dessa forma, toda raiz de multiplicidade $k$ em $p$ é uma raiz de multiplicidade pelo menos $k-1$ em $p^{\prime}$. Tome $\beta_{\ell}:=r_{\ell}(p)$ para todo $\ell \in[d-1] \operatorname{com} r_{\ell}(p)=r_{\ell+1}(p)$.

Seja $i \in[d-1]$ tal que $r_{i}(p)<r_{i+1}(p)$. Como $p(x) \neq 0$ para todo $x \in\left(r_{i}(p), r_{i+1}(p)\right)$, então, pelo Teorema do Valor Intermediário, temos que existe $\beta_{i} \in\left(r_{i}(p), r_{i+1}(p)\right)$ tal que $p^{\prime}\left(\beta_{i}\right)=0$.

Assim, vale que $\beta_{1}, \beta_{2}, \ldots, \beta_{d-1}$ são as raízes de $p^{\prime}$ tais que $\beta_{1} \leq \beta_{2} \leq \cdots \leq \beta_{d-1}$. Vale também que

$$
r_{1}(p) \leq \beta_{1} \leq r_{2}(p) \leq \beta_{2} \leq \cdots \leq \beta_{d-1} \leq r_{d}(p),
$$

e portanto, o polinômio $p^{\prime}$ entrelaça $p$.

Considere o polinômio $p(x)=x^{2}-9 x+18$ definido anteriormente. Note que $p(x)=(x-3)(x-6)$ e que $p^{\prime}(x)=2 x-9=2(x-4,5)$. Assim, é fácil ver que a raiz de $p^{\prime}$ entrelaça as raízes de $p$ e, portanto, o polinômio $p^{\prime}$ entrelaça $p$. Além disso, se existe um polinômio que entrelaça todos os polinômios de um conjunto de polinômios, dizemos que esse conjunto possui um entrelaçador comum.

Definição 4.3. Seja $Q \subseteq \mathbb{R}[x]$ um conjunto finito de polinômios de grau $d \geq 2$ que possuem somente raízes reais. Dizemos que $Q$ possui um entrelaçador comum se existe $p \in \mathbb{R}[x]$ com raízes reais tal que $p$ entrelaça $q$ para todo $q \in Q$.

Para exemplificar entrelaçadores comuns, considere novamente os polinômios $p, q \in \mathbb{R}[x]$ definidos em (4.1) por $p(x):=x^{2}-9 x+18$ e $q(x):=x^{3}-11 x^{2}+34 x-24$. Considere também o polinômio $r \in \mathbb{R}[x]$ definido por

$$
r(x):=x^{3}-9 x^{2}+18 x .
$$

Note que esse polinômio pode ser escrito como $r(x)=x p(x)$. Assim, é fácil ver que $p$ entrelaça os polinômios $q$ e $r$, e portanto $p$ é um entrelaçador comum do conjunto de polinômios $\{q, r\}$.

Uma das propriedades de conjuntos de polinômios que possuem um entrelaçador comum é que a raiz máxima de pelo menos um polinômio desse conjunto é limitada superiormente pela raiz máxima da soma de todos os polinômios desse conjunto.

Lema 4.4 ([24, Lema 4.2]). Seja $P \subseteq \mathbb{R}[x]$ um conjunto finito de polinômios com coeficientes dominantes positivos tal que $P$ possui um entrelaçador comum. Então existe $\bar{p} \in P$ tal que

$$
r_{\max }(\bar{p}) \leq r_{\max }\left(\sum_{p \in P} p\right) .
$$


Demonstração. Seja $q \in \mathbb{R}[x]$ um entrelaçador comum de $P$. Denote $s:=\sum_{p \in P} p$. Note que $r_{\max }(q) \leq r_{\max }(p)$ para todo $p \in P$. Assim, como os coeficientes dominantes dos polinômios de $P$ são positivos, então

$$
p\left(r_{\max }(q)\right) \leq 0 \quad \forall p \in P,
$$

o que implica que $s\left(r_{\max }(q)\right) \leq 0$. Note que o coeficiente dominante de $s$ também é positivo, e portanto, pelo Teorema do Valor Intermediário, temos que

$$
r_{\max }(q) \leq r_{\max }(s),
$$

como pode ser observado na Figura 4.1.

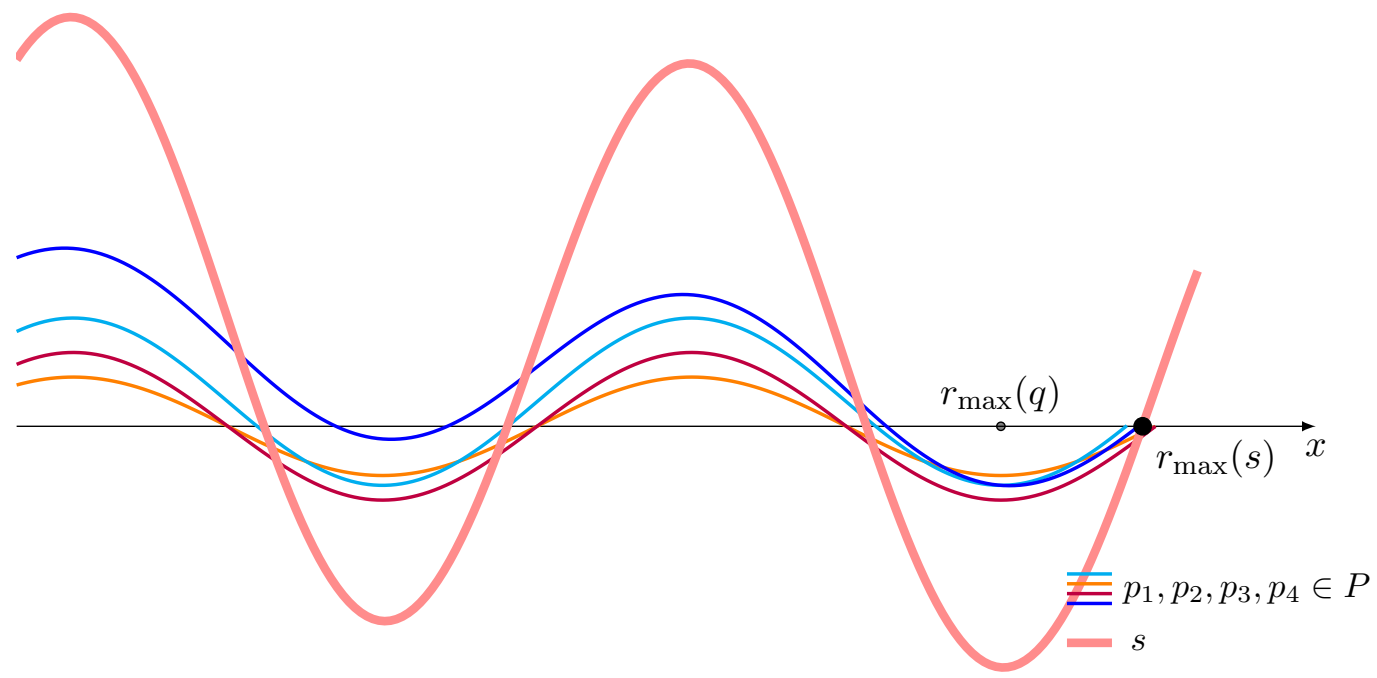

Figura 4.1: Representação dos polinômios do conjunto $P$, do polinômio $\sum_{p \in P} p$ e suas raízes máximas.

Se existe $\bar{p} \in P$ tal que $r_{\max }(\bar{p})=r_{\max }(q)$, então, por $(4.3)$, temos que $r_{\max }(\bar{p}) \leq r_{\max }(s)$.

Suponha que $r_{\max }(q)<r_{\max }(p)$ para todo $p \in P$. Note que $p(y)<0$ para todo $p \in P$ e todo $y \in\left(r_{\max }(q), r_{\max }(p)\right)$. Logo,

$$
s(y)<0 \quad \forall y \in\left(r_{\max }(q), \min _{p \in P} r_{\max }(p)\right) .
$$

Como $s$ possui coeficiente dominante positivo, então, pelo Teorema do Valor Intermediário, existe um número real $z \geq \min _{p \in P} r_{\max }(p)$ tal que $s(z)=0$, o que implica que

$$
\min _{p \in P} r_{\max }(p) \leq r_{\max }(s) .
$$

Portanto, existe $\bar{p} \in P$ tal que $r_{\max }(\bar{p}) \leq r_{\max }(s)$.

Considere os polinômios $\bar{q}, \bar{r} \in \mathbb{R}[x]$ definidos por

$$
\bar{q}(x):=x^{2}-5 x+4 \text { e } \bar{r}(x):=x^{2}-3 x .
$$

Note que $\bar{q}$ e $\bar{r}$ podem ser fatorados como

$$
\bar{q}(x)=(x-1)(x-4) \quad \text { e } \quad \bar{r}(x)=x(x-3) .
$$


Assim, vale que o polinômio $x-2$ entrelaça $\bar{q}$ e $\bar{r}$ e, portanto, o conjunto de polinômios $\{\bar{q}, \bar{r}\}$ possui um entrelaçador comum. Note também que

$$
(\bar{q}+\bar{r})(x)=2 x^{2}-8 x+4,
$$

e que as raízes do polinômio $\bar{q}+\bar{r}$ são $2-\sqrt{2}$ e $2+\sqrt{2}$. Assim, a raiz máxima de $\bar{q}+\bar{r}$ é pelo menos a raiz máxima de $\bar{r}$, ilustrando o Lema 4.4.

Para facilitar a prova de outras propriedades de conjuntos de polinômios que possuem um entrelaçador comum, introduziremos a seguir uma notação de intervalos entre raízes de um polinômio.

Notação 4.5. Seja $p \in \mathbb{R}[x]$ um polinômio de grau $d \geq 1$ que possui somente raízes reais. Denote $I_{p}^{j}:=\left[r_{j}(p), r_{j+1}(p)\right]$ para todo $j \in[d-1]$. Denote também $I_{p}^{0}:=\left(-\infty, r_{1}(p)\right]$ e $I_{p}^{d}:=\left[r_{d}(p),+\infty\right)$.

Também podemos dizer que um conjunto de polinômios possui um entrelaçador comum utilizando os intervalos entre as raízes dos polinômios desse conjunto. Isso se deve à caracterização de existência de entrelaçadores comuns apresentada a seguir.

Proposição 4.6. Seja $Q \subseteq \mathbb{R}[x]$ um conjunto finito de polinômios de grau $d \geq 2$ que possuem somente raízes reais. Então $Q$ possui um entrelaçador comum se e somente se $I_{p}^{j} \cap I_{q}^{j} \neq \varnothing$ para todo $j \in[d-1]$ e todos $p, q \in Q$.

Demonstração. Suponha que $Q$ possui um entrelaçador comum. Seja $p$ um entrelaçador comum de $Q$. Como $p$ entrelaça todos os polinômios de $Q$, então

$$
r_{1}(q) \leq r_{1}(p) \leq r_{2}(q) \leq r_{2}(p) \leq \cdots \leq r_{d-1}(p) \leq r_{d}(q)
$$

para todo $q \in Q$. Note que, para todo $j \in[d-1]$ e todo $q \in Q$, vale que $r_{j}(p) \in I_{q}^{j}$. Logo, para todo $j \in[d-1]$ e todos $p, q \in Q$, vale que

$$
I_{p}^{j} \cap I_{q}^{j} \neq \varnothing .
$$

Suponha agora que $I_{p}^{j} \cap I_{q}^{j} \neq \varnothing$ para todo $j \in[d-1]$ e todos $p, q \in Q$. Pelo Lema 2.6, vale que $\bigcap_{q \in Q} I_{q}^{j} \neq \varnothing$ para todo $j \in[d-1]$. Seja $r_{i} \in \bigcap_{q \in Q} I_{q}^{i}$ para todo $i \in[d-1]$. Seja $p \in \mathbb{R}[x]$ definido como

$$
p(x):=\prod_{i \in[d-1]}\left(x-r_{i}\right)
$$

Note que as raízes de $p$ entrelaçam as raízes de todos os polinômios de $Q$. Portanto, vale que $p$ é um entrelaçador comum de $Q$.

Considere novamente os polinômios $\bar{q}, \bar{r} \in \mathbb{R}[x]$ definidos em (4.4) como $\bar{q}(x):=x^{2}-5 x+4$ e $\bar{r}(x):=x^{2}-3 x$. Note que

$$
I_{\bar{q}}^{1}=[1,4] \quad \text { e } \quad I_{\bar{r}}^{1}=[0,3] .
$$

Logo, vale que $I_{\bar{q}}^{1} \cap I_{\bar{r}}^{1} \neq \varnothing$. Portanto, pela Proposição 4.6, temos que o conjunto de polinômios $\{\bar{q}, \bar{r}\}$ possui um entrelaçador comum, como também foi mostrado anteriormente.

Utilizaremos essa caracterização de existência de entrelaçadores comuns no resultado a seguir, que será utilizado na próxima seção para provar uma caracterização de árvores entrelaçadoras de polinômios.

Lema 4.7. Sejam $p, q \in \mathbb{R}[x]$ polinômios de grau $d \geq 2$ que possuem somente raízes reais tais que $\{p, q\}$ possui um entrelaçador comum. Se $\beta \in \mathbb{R}$ é uma raiz comum de $p$ e $q$, então o conjunto de polinômios $\left\{\frac{p}{x-\beta}, \frac{q}{x-\beta}\right\}$ possui um entrelaçador comum. 


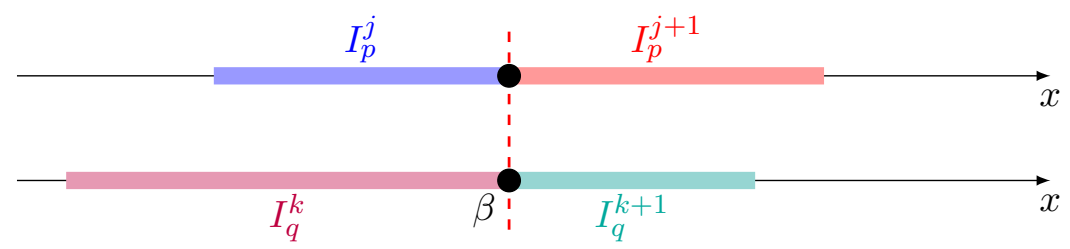

Figura 4.2: Representação dos conjuntos $I_{p}^{j}, I_{p}^{j+1}, I_{q}^{k}$ e $I_{q}^{k+1}$.

Demonstração. Suponha que $\beta \in \mathbb{R}$ é uma raiz de $p$ e $q$. Defina $\bar{p}:=\frac{p}{x-\beta}$ e $\bar{q}:=\frac{q}{x-\beta}$. Defina $j:=\min \left\{\ell \in\{0, \ldots, d-1\}: \beta \in I_{p}^{\ell}\right\}$ e $k:=\min \left\{\ell \in\{0, \ldots, d-1\}: \beta \in I_{q}^{\ell}\right\}$, como mostrado na Figura 4.2 .

Suponha, sem perda de generalidade, que $j \leq k$. Pela Proposição 4.6, temos que $I_{p}^{j} \cap I_{q}^{j} \neq \varnothing$ e que $I_{p}^{j+1} \cap I_{q}^{j+1} \neq \varnothing$, o que implica que $\beta \in I_{q}^{j} \cup I_{q}^{j+1}$, como mostrado na Figura 4.3. Portanto, como $j \leq k$, então $k=j$ ou $k=j+1$.

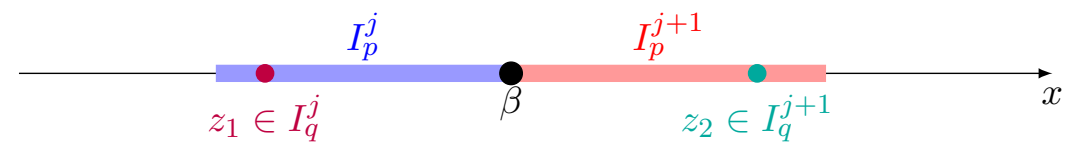

Figura 4.3: Representação das intersecções não vazias $\left(I_{p}^{j} \cap I_{q}^{j}\right) \ni z_{1}$ e $\left(I_{p}^{j+1} \cap I_{q}^{j+1}\right) \ni z_{2}$.

Note que, para todo $\ell \in[d-2]$, vale que

$$
I_{\bar{p}}^{\ell}=\left\{\begin{array}{ll}
I_{p}^{\ell} & \text { se } \ell<j, \\
I_{p}^{\ell+1} & \text { se } \ell>j, \\
I_{p}^{j} \cup I_{p}^{j+1} & \text { se } \ell=j,
\end{array} \quad \text { e } \quad I_{\bar{q}}^{\ell}= \begin{cases}I_{q}^{\ell} & \text { se } \ell<k, \\
I_{q}^{\ell+1} & \text { se } \ell>k, \\
I_{q}^{k} \cup I_{q}^{k+1} & \text { se } \ell=k .\end{cases}\right.
$$

Se $k=j$, então, pela Proposição 4.6, temos que

$$
I_{\bar{p}}^{j} \cap I_{\bar{q}}^{j}=\left(I_{p}^{j} \cup I_{p}^{j+1}\right) \cap\left(I_{q}^{j} \cup I_{q}^{j+1}\right) \supseteq I_{p}^{j} \cap I_{q}^{j} \neq \varnothing .
$$

Caso contrário, vale que $k=j+1$, e então, pela Proposição 4.6, temos que

$$
\begin{aligned}
I_{\bar{p}}^{j} \cap I_{\bar{q}}^{j} & =\left(I_{p}^{j} \cup I_{p}^{j+1}\right) \cap I_{q}^{j} \supseteq I_{p}^{j} \cap I_{q}^{j} \neq \varnothing, \\
I_{\bar{p}}^{k} \cap I_{\bar{q}}^{k} & =I_{p}^{k+1} \cap\left(I_{q}^{k} \cup I_{q}^{k+1}\right) \supseteq I_{p}^{k+1} \cap I_{q}^{k+1} \neq \varnothing .
\end{aligned}
$$

Assim, vale que $I_{\bar{p}}^{\ell} \cap I_{\bar{q}}^{\ell} \neq \varnothing$ para todo $\ell \in[d-2]$. Logo, pela Proposição 4.6, temos que $\{\bar{p}, \bar{q}\}$ possui um entrelaçador comum.

Considere os polinômios $q, r \in \mathbb{R}[x]$ definidos anteriormente em (4.1) e (4.2) como

$$
q(x):=x^{3}-11 x^{2}+34 x-24 \text { e } r(x):=x^{3}-9 x^{2}+18 x .
$$

Note que $q(x)=(x-1)(x-4)(x-6)$ e $r(x)=x(x-3)(x-6)$. Considere também os polinômios $\bar{q}, \bar{r} \in \mathbb{R}[x]$ definidos em $(4.4)$ como $\bar{q}(x):=x^{2}-5 x+4$ e $\bar{r}(x):=x^{2}-3 x$. Note que $\frac{q}{x-6}=\bar{q}$ e $\frac{r}{x-6}=\bar{r}$. Pelo Lema 4.7, temos que $\left\{\frac{q}{x-6}, \frac{r}{x-6}\right\}=\{\bar{q}, \bar{r}\}$ possui um entrelaçador comum, como mostrado anteriormente.

Definiremos agora o entrelaçamento estrito de polinômios, que é basicamente o entrelaçamento de polinômios de forma que a desigualdade entre as raízes dos polinômios é estrita. 
Definição 4.8. Sejam $p, q \in \mathbb{R}[x]$ polinômios de graus $d-1$ e $d \geq 2$, respectivamente, que possuem somente raízes reais. Dizemos que $p$ entrelaça estritamente o polinômio $q$ se

$$
r_{1}(q)<r_{1}(p)<r_{2}(q)<r_{2}(p)<\cdots<r_{d-1}(p)<r_{d}(q) .
$$

Considere os polinômios $p, q \in \mathbb{R}[x]$ definidos em (4.1) como

$$
p(x):=x^{2}-9 x+18 \quad \text { e } \quad q(x):=x^{3}-11 x^{2}+34 x-24 .
$$

Note que $p(x)=(x-3)(x-6)$ e $q(x)=(x-1)(x-4)(x-6)$. Assim, é fácil ver que as raízes de $p$ e $q$ se entrelaçam, porém não se entrelaçam estritamente. Portanto, $p$ entrelaça $q$, mas não estritamente. Mostraremos agora uma característica do entrelaçamento estrito: se todos os polinômios de um conjunto de polinômios entrelaçam estritamente um polinômio $p$, então a soma de todos os polinômios desse conjunto também entrelaça estritamente o polinômio $p$. Essa propriedade será utilizada no Capítulo 6 para mostrar que os polinômios de empalhamentos de grafos completos possuem somente raízes reais.

Lema 4.9. Seja $p \in \mathbb{R}[x]$ um polinômio de grau $d \geq 2$ que possui somente raízes reais. Seja $\varnothing \neq Q \subseteq \mathbb{R}[x]$ um conjunto finito de polinômios que possuem somente raízes reais e coeficientes dominantes de mesmo sinal tais que $q$ entrelaça estritamente $p$ para todo $q \in Q$. Então o polinômio $\sum_{q \in Q} q$ entrelaça estritamente $p$.

Demonstração. Seja $j \in[d-1]$. Como todos os polinômios de $Q$ entrelaçam estritamente $p$ e seus coeficientes dominantes possuem o mesmo sinal, então $p\left(r_{j}(q)\right)>0$ para todo $q \in Q$ ou $p\left(r_{j}(q)\right)<0$ para todo $q \in Q$.

Suponha, sem perda de generalidade, que $p\left(r_{j}(q)\right)>0$ para todo $q \in Q$. Assim, $q(x)>0$ para

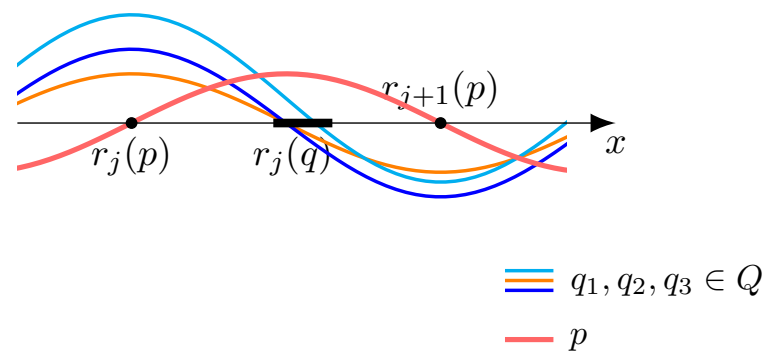

Figura 4.4: Representação do polinômio $p$, dos polinômios do conjunto $Q$ e de suas raízes.

todo $q \in Q$ e todo $x \in\left(r_{j}(p), r_{j}(q)\right)$, como mostrado na Figura 4.4. Logo, vale que

$$
\sum_{q \in Q} q(x)>0
$$

para todo $x \in\left(r_{j}(p), \min _{q \in Q} r_{j}(q)\right)$. Também vale que $q(x)<0$ para todo $q \in Q$ e todo $x \in$ $\left(r_{j}(q), r_{j+1}(p)\right)$, o que implica que

$$
\sum_{q \in Q} q(x)<0
$$

para todo $x \in\left(\max _{q \in Q} r_{j}(q), r_{j+1}(p)\right)$. Portanto, pelo Teorema do Valor Intermediário, existe $x \in\left(r_{j}(p), r_{j+1}(p)\right)$ tal que $\sum_{q \in Q} q(x)=0$. 


\subsection{Famílias entrelaçadoras de polinômios}

Nesta seção, apresentaremos árvores entrelaçadoras de polinômios, que são árvores cujas folhas representam polinômios com coeficientes dominantes positivos e cujos nós internos representam a soma dos polinômios representados pelos seus nós filhos. Além disso, todos os polinômios filhos de um mesmo nó possuem um entrelaçador comum. Apesar de não possuir necessariamente um entrelaçador comum, o conjunto de polinômios representados pelas folhas de uma árvore entrelaçadora de polinômios possui uma das propriedades de conjuntos que possuem um entrelaçador comum: a raiz máxima da soma de todos os polinômios representados pelas folhas de uma árvore entrelaçadora de polinômios limita superiormente a raiz máxima do polinômio representado por uma das folhas dessa árvore. Note que os conjuntos que possuem um entrelaçador comum também se comportam dessa forma, como mostrado no Lema 4.4. Por satisfazer essa propriedade, essas árvores serão utilizadas ao longo do texto para provar a existência de famílias infinitas de grafos de Ramanujan, e também para provar uma resolução do Problema de Kadison-Singer.

Definição 4.10 (Árvore de polinômios). Sejam $T$ uma arborescência e $f: V(T) \rightarrow \mathbb{R}[x]$ tal que $f(v)$ é um polinômio que possui somente raízes reais e coeficiente dominante positivo para todo $v \in V(T)$. Dizemos que $(T, f)$ é uma árvore de polinômios se $f(v)=\sum_{u \in N_{\text {out }}(v)} f(u)$ para todo $v \in V \operatorname{com} N_{\text {out }}(v) \neq \varnothing$.

Definição 4.11 (Árvore entrelaçadora de polinômios). Seja $(T, f)$ uma árvore de polinômios. Dizemos que $(T, f)$ é uma árvore entrelaçadora de polinômios se $f\left(N_{\text {out }}(v)\right)$ possui um entrelaçador comum para todo $v \in V \operatorname{com} N_{\text {out }}(v) \neq \varnothing$.

Note que árvores entrelaçadoras de polinômios na verdade são arborescências. Escolhemos representar essas árvores assim pois a notação associada a arborescências é mais natural na sua definição.

Para uma noção mais intuitiva, segue a Figura 4.5, que é um exemplo de árvore entrelaçadora de polinômios, onde

$$
\begin{aligned}
p_{(0,0)} & :=x^{3}-15 x^{2}+62 x-48, & p_{(0,1)} & :=x^{3}-16 x^{2}+69 x-54, \\
p_{(0,2)} & :=x^{3}-17 x^{2}+76 x-60, & p_{(1,0)} & :=x^{3}-15 x^{2}+59 x-45, \\
p_{(1,1)} & :=x^{3}-16 x^{2}+69 x-54, & p_{(0)} & :=3 x^{3}-48 x^{2}+207 x-162, \\
p_{(1)} & :=2 x^{3}-31 x^{2}+128 x-99, & p_{\varnothing} & :=5 x^{3}-79 x^{2}+335 x-261 .
\end{aligned}
$$

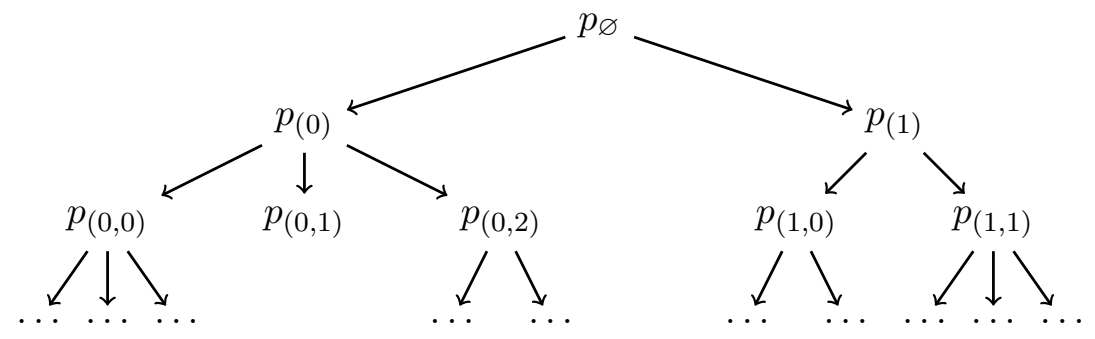

Figura 4.5: Representação de uma árvore entrelaçadora de polinômios.

Note que os polinômios representados pelos nós da árvore entrelaçadora de polinômios da Figura 4.5 podem ser escritos da seguinte maneira:

$$
p_{(0,0)}=(x-1)(x-6)(x-8), \quad p_{(0,1)}=(x-1)(x-6)(x-9),
$$




$$
\begin{aligned}
p_{(0,2)} & =(x-1)(x-6)(x-10), & p_{(1,0)} & =(x-1)(x-5)(x-9), \\
p_{(1,1)} & =(x-1)(x-6)(x-9), & p_{(0)} & =3(x-1)(x-6)(x-9), \\
p_{(1)} & =2(x-1)(x-5.5)(x-9), & & p_{(\varnothing)}=5(x-1)(x-5.8)(x-9) .
\end{aligned}
$$

Por ser uma árvore entrelaçadora de polinômios, cada nó interno dessa árvore representa a soma de todos os polinômios representados pelos seus filhos. Além disso, todos os polinômios representados pelos filhos de um mesmo nó possuem um entrelaçador comum. Assim, no exemplo da Figura 4.5, pode-se observar que

$$
\begin{aligned}
p_{\varnothing} & =p_{(0)}+p_{(1)}, \\
p_{(0)} & =p_{(0,0)}+p_{(0,1)}+p_{(0,2)}, \\
p_{(1)} & =p_{(1,0)}+p_{(1,1)} .
\end{aligned}
$$

Também vale que o conjunto de polinômios $\left\{p_{(0)}, p_{(1)}\right\}$ possui um entrelaçador comum, assim como o conjunto $\left\{p_{(0,0)}, p_{(0,1)}, p_{(0,2)}\right\}$, e também como o conjunto $\left\{p_{(1,0)}, p_{(1,1)}\right\}$.

Deixamos mais exemplos de árvores entrelaçadoras de polinômios para o Capítulo 5, pois iremos utilizar uma classe especial de polinômios, chamada de polinômios estáveis, para provar que algumas árvores de polinômios são entrelaçadoras. Portanto, trataremos os exemplos dessas árvores como uma aplicação de polinômios estáveis no próximo capítulo.

Agora, apresentaremos um resultado de Marcus, Spielman e Srivastava [24], que mostra que os polinômios representados pelas folhas de árvores entrelaçadoras de polinômios satisfazem uma propriedade que vale para conjuntos de polinômios que possuem um entrelaçador comum, como mostrado no Lema 4.4 .

Corolário 4.12 (Marcus, Spielman e Srivastava [24]). Seja $(T, f)$ uma árvore entrelaçadora de polinômios e seja $F:=\left\{u \in V(T): N_{\text {out }}(u)=\varnothing\right\}$ o conjunto de folhas de $T$. Então existe $v \in F$ tal que

$$
r_{\max }(f(v)) \leq r_{\max }\left(\sum_{u \in F} f(u)\right) .
$$

Demonstração. Seja $U:=V(T) \backslash F$ o conjunto de nós internos de $T$. Como $(T, f)$ é uma árvore entrelaçadora de polinômios, então, para todo $u \in U$, vale que o conjunto de polinômios

$$
\left\{f(w): w \in N_{\text {out }}(u)\right\}
$$

possui um entrelaçador comum. Logo, pelo Lema 4.4, temos que, para todo $u \in U$, existe $w \in$ $N_{\text {out }}(u)$ tal que

$$
r_{\max }(f(w)) \leq r_{\max }(f(u)) .
$$

Logo, existe $v \in F$ tal que

$$
r_{\max }(f(v)) \leq r_{\max }(f(r(T)))=r_{\max }\left(\sum_{u \in F} f(u)\right) .
$$

Neste texto, utilizamos árvores entrelaçadoras de polinômios cujos nós de mesma altura possuem todos a mesma quantidade de filhos. Chamaremos essas árvores de árvores entrelaçadoras de polinômios balanceadas. Para facilitar o entendimento de alguns resultados nos próximos capítulos, definiremos a seguir a família de polinômios representados pelas folhas de árvores entrelaçadoras de polinômios balanceadas. 
Definição 4.13 (Família entrelaçadora de polinômios). Sejam $S_{1}, S_{2}, \ldots, S_{m}$ conjuntos finitos e seja $p_{s}$ um polinômio com raízes reais e coeficiente dominante positivo para todo $s \in S_{1} \times S_{2} \times \cdots \times S_{m}$. Defina $S^{0}:=\{\varnothing\}$ e defina $S^{i}:=S_{1} \times S_{2} \times \cdots \times S_{i}$ para todo $i \in[m]$. Seja $T=(V, A)$ uma arborescência tal que $V=\bigcup_{i=0}^{m} S^{i}$ e

$$
A=\left\{\left(\left(s_{1}, \ldots, s_{i-1}\right),\left(s_{1}, \ldots, s_{i-1}, s_{i}\right)\right) \in S^{i-1} \times S^{i}: i \in[m]\right\} .
$$

Seja $(T, f)$ a árvore de polinômios tal que $f(s)=p_{s}$ para todo $s \in S^{m}$. Dizemos que a família de polinômios $\left\{p_{s}\right\}_{s \in S^{m}}$ é uma família entrelaçadora de polinômios se $(T, f)$ é uma árvore entrelaçadora de polinômios.

Utilizamos a Figura 4.6 para ilustrar o conceito de famílias entrelaçadoras de polinômios. Note

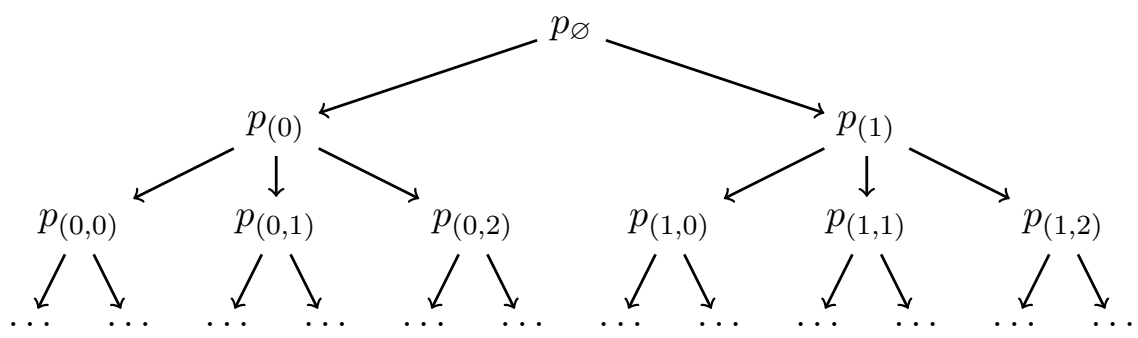

Figura 4.6: Representação de uma árvore entrelaçadora de polinômios.

que, na árvore representada na Figura 4.6, todos os nós de mesma altura possuem o mesmo número de filhos, o que não é exigido na definição de árvores entrelaçadoras de polinômios.

\subsection{Provando que uma árvore de polinômios é entrelaçadora}

Para provar que uma determinada árvore de polinômios é entrelaçadora, é necessário mostrar que determinados conjuntos de polinômios dessa árvore possuem um entrelaçador comum, o que poderia ser feito encontrando um entrelaçador comum para cada um desses conjuntos. Porém, em alguns casos, essa não é uma tarefa simples. Por esse motivo, utilizamos uma caracterização de conjuntos que possuem um entrelaçador comum: um conjunto de polinômios possui um entrelaçador comum se e somente se todas as combinações convexas desses polinômios possuem somente raízes reais. Essa caracterização foi provada por Chudnovsky e Seymour [8], Dedieu [9] e Fell [10]. Seguiremos a prova de Chudnovsky e Seymour. Para provar essa caracterização, utilizamos um Teorema de Hurwitz [28], que não será provado neste texto.

Dessa forma, para mostrar que uma família de polinômios é uma família entrelaçadora de polinômios, basta mostrar que combinações convexas dos polinômios dessa família, com certos suportes, possuem somente raízes reais. Mostraremos alguns exemplos de famílias entrelaçadoras de polinômios, no próximo capítulo, utilizando essa caracterização de Chudnovsky e Seymour juntamente com algumas ferramentas de polinômios estáveis.

\subsubsection{Ferramentas de análise}

Sejam $\Omega \subseteq \mathbb{C}$ e $p \in \mathbb{C}[x]$. Dizemos que uma sequência de polinômios $\left(p_{n}\right)_{n \in \mathbb{N}}$ em $\mathbb{C}[x]$ converge para o polinômio $p$ em $\Omega$ se, para todo $y \in \Omega$ e todo $\varepsilon>0$ existe $N \in \mathbb{N}$ tal que, para todo $n \in \mathbb{N}$ com $n \geq N$, vale que $\left|p_{n}(y)-p(y)\right|<\varepsilon$. Dizemos também que a sequência $\left(p_{n}\right)_{n \in \mathbb{N}}$ em $\mathbb{C}[x]$ converge 
uniformemente para o polinômio $p$ em $\Omega$ se, para todo $\varepsilon>0$ existe $N \in \mathbb{N}$ tal que, para todo $y \in \Omega$ e todo $n \in \mathbb{N}$ com $n \geq N$, vale que $\left|p_{n}(y)-p(y)\right|<\varepsilon$. Defina $N_{\varepsilon}(y):=\{z \in \mathbb{C}:|z-y|<\varepsilon\}$ para todos $\varepsilon \in \mathbb{R}_{++}$e $y \in \mathbb{C}$.

Teorema 4.14 (Hurwitz (vide [28, Teorema 1.3.8])). Seja $\varnothing \neq \Omega \subseteq \mathbb{C}$ aberto e conexo. Sejam $y \in \Omega$ e $\left(p_{n}\right)_{n \in \mathbb{N}}$ uma sequência de polinômios em $\mathbb{R}[x]$ que converge uniformemente para um polinômio $p \in \mathbb{R}[x]$ em todo subconjunto compacto de $\Omega$. Então $y$ é uma raiz de $p$ de multiplicidade $m$ se e somente se existe $\delta>0$ tal que para todo $\varepsilon \in(0, \delta)$, existe $N \in \mathbb{N} \backslash\{0\}$ tal que para todo $n \in \mathbb{N}$ com $n \geq N$, vale que $p_{n}$ possui $m$ raízes em $\Omega \cap N_{\varepsilon}(y)$, considerando multiplicidades.

Corolário 4.15. Seja $\Omega \subseteq \mathbb{C}$ aberto e conexo. Seja $p \in \mathbb{R}[x]$ e sejam $p_{n} \in \mathbb{R}[x]$ para todo $n \in \mathbb{N}$ tais que $\left(p_{n}\right)_{n \in \mathbb{N}}$ converge uniformemente para $p$ em todo subconjunto compacto de $\Omega$. Se $p_{n}(y) \neq 0$ para todo $n \in \mathbb{N}$ e todo $y \in \Omega$, então $p=0$ ou $p(y) \neq 0$ para todo $y \in \Omega$.

Demonstração. Suponha que $p_{n}(y) \neq 0$ para todo $n \in \mathbb{N}$ e todo $y \in \Omega$. Suponha também que $p \neq 0$ e que existe $w \in \Omega$ tal que $p(w)=0$.

Pelo Teorema 4.14, existem $\varepsilon>0$ e $N \in \mathbb{N} \backslash\{0\}$ tal que para todo $n \in \mathbb{N}$ com $n \geq N$, vale que existe $y \in \Omega \cap N_{\varepsilon}(w)$ tal que $p_{n}(y)=0$. Como $\Omega \cap N_{\varepsilon}(w) \neq \varnothing$, então existe $y \in \Omega$ e $n \in \mathbb{N}$ tais que $p_{n}(y)=0$, o que nos leva a uma contradição.

Teorema 4.16. Seja $X \subseteq \mathbb{C}$ compacto. Sejam $p, p_{n} \in \mathbb{C}[x]$ polinômios de grau $d \geq 1$ para todo $n \in \mathbb{N}$. Sejam $c, c_{n}:\{0, \ldots, d\} \rightarrow \mathbb{C}$ tais que $p(x)=\sum_{i=0}^{d} c(i) x^{i}$ e $p_{n}(x)=\sum_{i=0}^{d} c_{n}(i) x^{i}$ para todo $n \in \mathbb{N}$. Se $\lim _{n \rightarrow \infty} c_{n}(i)=c(i)$ para todo $i \in\{0, \ldots, d\}$, então $\left(p_{n}\right)_{n \in \mathbb{N}}$ converge uniformemente para $p$ em $X$.

Demonstração. Se $X=\{0\}$, é fácil ver que o resultado vale.

Suponha que $X \neq\{0\}$. Suponha também que $\lim _{n \rightarrow \infty} c_{n}(i)=c(i)$ para todo $i \in\{0, \ldots, d\}$. Seja $n \in \mathbb{N}$. Defina $\alpha:=\max \left\{\left|y^{i}\right|: y \in X\right.$ e $\left.i \in\{0, \ldots, d\}\right\}$. Como $X \neq\{0\}$, então $\alpha>0$.

Note que, para todo $y \in X$, vale que

$$
\begin{aligned}
\left|p_{n}(y)-p(y)\right| & =\left|\sum_{i=0}^{d} c_{n}(i) y^{i}-\sum_{i=0}^{d} c(i) y^{i}\right| \\
& =\left|\sum_{i=0}^{d}\left(c_{n}(i)-c(i)\right) y^{i}\right| \\
& \leq \sum_{i=0}^{d}\left|\left(c_{n}(i)-c(i)\right) y^{i}\right| \\
& =\sum_{i=0}^{d}\left|\left(c_{n}(i)-c(i)\right)\right|\left|y^{i}\right| \\
& \leq \alpha \sum_{i=0}^{d}\left|\left(c_{n}(i)-c(i)\right)\right|
\end{aligned}
$$

Logo, vale que $\left|p_{n}(y)-p(y)\right| \leq \alpha \sum_{i=0}^{d}\left|\left(c_{n}(i)-c(i)\right)\right|$ para todo $y \in X$.

Seja $\varepsilon>0$. Como $\lim _{n \rightarrow \infty} c_{n}(i)=c(i)$ para todo $i \in\{0, \ldots, d\}$, então, para todo $i \in\{0, \ldots, d\}$, existe $N_{i} \in \mathbb{N}$ tal que

$$
\left|c_{n}(i)-c(i)\right|<\frac{\varepsilon}{\alpha d}
$$


para todo $n \in \mathbb{N}$ com $n \geq N_{i}$. Defina $N:=\max _{i \in\{0, \ldots, d\}} N_{i}$. Note que $\left|c_{n}(i)-c(i)\right|<\frac{\varepsilon}{\alpha d}$ para todo $i \in\{0, \ldots, d\}$ e todo $n \in \mathbb{N}$ com $n \geq N$. Assim, vale que $\alpha \sum_{i=0}^{d}\left|\left(c_{n}(i)-c(i)\right)\right|<\varepsilon$ para todo $n \in \mathbb{N}$ com $n \geq N$. Logo, para todo $y \in X$, vale que

$$
\left|p_{n}(y)-p(y)\right|<\varepsilon
$$

para todo $n \in \mathbb{N}$ com $n \geq N$. Portanto, a sequência de polinômios $\left(p_{n}\right)_{n \in \mathbb{N}}$ converge uniformemente para $p$ em $X$.

Lema 4.17. Seja $X \subseteq \mathbb{R}$. Seja $p_{\lambda} \in \mathbb{R}[x]$ um polinômio de grau $d \geq 1$ que possui somente raízes reais para todo $\lambda \in X$. Seja $i \in[d]$ e seja $\beta: X \rightarrow \mathbb{R}$ definida como $\lambda \mapsto r_{i}\left(p_{\lambda}\right)$. Se, para todo $\lambda \in X$ e para toda sequência $\left(\mu_{n}\right)_{n \in \mathbb{N}}$ em $X-\lambda \operatorname{com} \lim _{n \rightarrow \infty} \mu_{n}=0$, a sequência $\left(p_{\lambda+\mu_{n}}\right)_{n \in \mathbb{N}}$ converge uniformemente para $p_{\lambda}$ em todo subconjunto compacto de $\mathbb{C}$, então $\beta$ é uma função contínua.

Demonstração. Sejam $\lambda \in X$ e $\left(\mu_{n}\right)_{n \in \mathbb{N}}$ uma sequência em $X-\lambda$ com $\lim _{n \rightarrow \infty} \mu_{n}=0$ tal que $\left(p_{\lambda+\mu_{n}}\right)_{n \in \mathbb{N}}$ converge uniformemente para $p_{\lambda}$ em todo subconjunto compacto de $\mathbb{C}$. Seja $\gamma \in$ $[-\infty,+\infty]$ definido como $\gamma:=+\infty$ se $r_{1}\left(p_{\lambda}\right)=r_{d}\left(p_{\lambda}\right)$ e

$$
\gamma:=\min _{j \in[d-1]}\left\{\frac{\left|r_{j}\left(p_{\lambda}\right)-r_{j+1}\left(p_{\lambda}\right)\right|}{3}: r_{j}\left(p_{\lambda}\right) \neq r_{j+1}\left(p_{\lambda}\right)\right\}
$$

caso contrário. Seja $k \in[d]$ e seja $m$ a multiplicidade de $r_{k}\left(p_{\lambda}\right)$ em $p_{\lambda}$. Pelo Teorema 4.14 , existe $\delta>0$ tal que, para todo $\varepsilon \in(0, \min \{\delta, \gamma\})$, existem $N \in \mathbb{N} \backslash\{0\}$ e $j_{1}, j_{2}, \ldots, j_{m} \in[d]$ distintos tais que

$$
\left|r_{k}\left(p_{\lambda}\right)-r_{j_{\ell}}\left(p_{\lambda+\mu_{n}}\right)\right|<\varepsilon
$$

para todo natural $n \geq N$ e todo $\ell \in[m]$. Note que

$$
N_{\gamma}\left(r_{k_{1}}\left(p_{\lambda}\right)\right) \cap N_{\gamma}\left(r_{k_{2}}\left(p_{\lambda}\right)\right)=\varnothing
$$

para todo $k_{1}, k_{2} \in[d] \operatorname{com} r_{k_{1}}\left(p_{\lambda}\right) \neq r_{k_{2}}\left(p_{\lambda}\right)$. Dessa forma, existe $\ell \in[m]$ tal que $j_{\ell}=k$.

Logo, para toda sequência $\left(\mu_{n}\right)_{n \in \mathbb{N}}$, existe $\delta>0$ tal que, para todo $\varepsilon \in(0, \min \{\delta, \gamma\})$, existe $N \in \mathbb{N} \backslash\{0\}$ tal que

$$
\left|\beta(\lambda)-\beta\left(\lambda+\mu_{n}\right)\right|=\left|r_{i}\left(p_{\lambda}\right)-r_{i}\left(p_{\lambda+\mu_{n}}\right)\right|<\varepsilon
$$

para todo $n \in \mathbb{N}$ com $n \geq N$.

Assim, para toda sequência $\left(\mu_{n}\right)_{n \in \mathbb{N}}$ em $X-\lambda \operatorname{com} \lim _{n \rightarrow \infty} \mu_{n}=0$, vale que

$$
\lim _{n \rightarrow \infty} \beta\left(\lambda+\mu_{n}\right)=\beta(\lambda) .
$$

Logo, pela Proposição 2.5, temos que $\lim _{\mu \rightarrow \lambda} \beta(\mu)=\beta(\lambda)$. Portanto, vale que $\beta$ é contínua em $X$.

Lema 4.18 (Continuidade de autovalores). Sejam $A, B \in \mathbb{C}^{d \times d}$ matrizes hermitianas com $B \succ 0$ e $d \geq 1$. Seja $i \in[d]$. Seja $\lambda_{A, B}: \mathbb{R} \rightarrow \mathbb{R}$ definida como $t \mapsto \lambda_{i}^{\uparrow}(A+t B)$. Vale que $\lambda_{A, B}$ é contínua em $\mathbb{R}$.

Demonstração. Seja $p_{t} \in \mathbb{C}[x]$ tal que $p_{t}(x):=\operatorname{det}(x I-A-t B)$ para todo $t \in \mathbb{R}$. Note que $\lambda_{A, B}(t)=r_{i}\left(p_{t}\right)$ para todo $t \in \mathbb{R}$. Pela Proposição 2.24 , temos que $p_{t}$ possui somente raízes reais para todo $t \in \mathbb{R}$. 
Seja $t \in \mathbb{R}$. Seja $\left(\mu_{n}\right)_{n \in \mathbb{N}}$ uma sequência de números reais tais que $\lim _{n \rightarrow \infty} \mu_{n}=0$. Note que

$$
\lim _{n \rightarrow \infty}\left[x^{j}\right]\left(p_{t+\mu_{n}}\right)=\left[x^{j}\right]\left(p_{t}\right)
$$

para todo $j \in[d]$. Assim, pelo Teorema 4.16, temos que a sequência $\left(p_{t+\mu_{n}}\right)_{n \in \mathbb{N}}$ converge uniformemente para $p_{t}$ em todo subconjunto compacto de $\mathbb{C}$.

Portanto, pelo Lema 4.17, temos que $\lambda_{A, B}$ é uma função contínua em $\mathbb{R}$.

Lema 4.19. Sejam $p_{1}, p_{2} \in \mathbb{R}[x]$ polinômios de grau $d \geq 1$ tais que $\lambda p_{1}+(1-\lambda) p_{2}$ possui somente raízes reais para todo $\lambda \in[0,1]$. Seja $i \in[d]$ e Seja $\beta:[0,1] \rightarrow \mathbb{R}$ definido como

$$
\beta(\lambda):=r_{i}\left(\lambda p_{1}+(1-\lambda) p_{2}\right)
$$

para todo $\lambda \in[0,1]$. Então $\beta$ é uma função contínua em $[0,1]$.

Demonstração. Defina $q_{\lambda}:=\lambda p_{1}+(1-\lambda) p_{2}$ para todo $\lambda \in[0,1]$.

Seja $\lambda \in[0,1]$. Seja $\left(\mu_{n}\right)_{n \in \mathbb{N}}$ uma sequência monótona de números reais em $[-\lambda, 1-\lambda]$ tal que $\lim _{n \rightarrow \infty} \mu_{n}=0$.

Note que $\lim _{n \rightarrow \infty}\left[x^{j}\right]\left(q_{\lambda+\mu_{n}}\right)=\left[x^{j}\right]\left(q_{\lambda}\right)$ para todo $j \in[d]$. Assim, pelo Teorema 4.16, temos que a sequência $\left(q_{\lambda+\mu_{n}}\right)_{n \in \mathbb{N}}$ converge uniformemente para $q_{\lambda}$ em todo subconjunto compacto de $\mathbb{C}$.

Assim, para todo $\lambda \in[0,1]$ e toda sequência monótona $\left(\mu_{n}\right)_{n \in \mathbb{N}}$ em $[-\lambda, 1-\lambda] \operatorname{com} \lim _{n \rightarrow \infty} \mu_{n}=0$, vale que $\left(q_{\lambda+\mu_{n}}\right)_{n \in \mathbb{N}}$ converge uniformemente para $q_{\lambda}$ em todo subconjunto compacto de $\mathbb{C}$. Logo, pelo Lema 4.17, temos que $\beta$ é uma função contínua em $[0,1]$.

\subsubsection{Raízes de combinações convexas de polinômios}

Notação 4.20. Seja $p \in \mathbb{R}[x]$ um polinômio de grau $d$ de raízes reais e sejam $\alpha, \beta \in[-\infty,+\infty]$ $\operatorname{com} \alpha<\beta$. Denote

$$
n_{p}[\alpha, \beta]:=\left|\left\{i \in[d]: r_{i}(p) \in[\alpha, \beta]\right\}\right| .
$$

Lema 4.21 ([8, Teorema 3.3]). Sejam $p, q \in \mathbb{R}[x]$ polinômios de mesmo grau tais que o polinômio $\lambda p+(1-\lambda) q$ possui somente raízes reais para todo $\lambda \in[0,1]$. Sejam $\alpha, \beta \in \mathbb{R}$ tais que $p(\alpha) q(\alpha)>0$ e $p(\beta) q(\beta)>0$. Então $n_{p}[\alpha, \beta]=n_{q}[\alpha, \beta]$.

Demonstração. Seja $\bar{p}_{\lambda}:=\lambda p+(1-\lambda) q$ para todo $\lambda \in[0,1]$. Como $p(\alpha) q(\alpha)>0$ e $p(\beta) q(\beta)>0$, então $\bar{p}_{\lambda}(\alpha) \neq 0$ e $\bar{p}_{\lambda}(\beta) \neq 0$ para todo $\lambda \in[0,1]$. Seja $d$ o grau de $p$ e $q$.

Pelo Lema 4.19, temos que $r_{1}\left(\bar{p}_{\lambda}\right), r_{2}\left(\bar{p}_{\lambda}\right), \ldots, r_{d}\left(\bar{p}_{\lambda}\right)$ são funções contínuas. Assim, se existe $i \in[d]$ tal que $r_{i}\left(\bar{p}_{0}\right) \in(\alpha, \beta)$, então $r_{i}\left(\bar{p}_{\lambda}\right) \in(\alpha, \beta)$ para todo $\lambda \in[0,1]$. Logo, vale que

$$
n_{\bar{p}_{\lambda}}[\alpha, \beta]=n_{\bar{p}_{\mu}}[\alpha, \beta]
$$

para todo $\lambda, \mu \in[0,1]$. Portanto $n_{p}[\alpha, \beta]=n_{\bar{p}_{1}}[\alpha, \beta]=n_{\bar{p}_{0}}[\alpha, \beta]=n_{q}[\alpha, \beta]$.

Lema 4.22 ([8, Teorema 3.4]). Sejam $p, q \in \mathbb{R}[x]$ polinômios de mesmo grau e com coeficientes dominantes positivos tais que $\lambda p+(1-\lambda) q$ possui somente raízes reais para todo $\lambda \in[0,1]$. Então

$$
\left|n_{p}[-\infty, y]-n_{q}[-\infty, y]\right| \leq 1
$$

para todo $y \in \mathbb{R}$. 
Demonstração. Seja $d$ o grau de $p$ e $q$. Provaremos esse resultado por indução em $d$.

Se $d=1$, é fácil ver que o resultado vale.

Suponha que $d>1$. Suponha por contradição que existe $y \in \mathbb{R}$ tal que

$$
\left|n_{p}[-\infty, y]-n_{q}[-\infty, y]\right| \geq 2 .
$$

Suponha, sem perda de generalidade, que $n_{p}[-\infty, y]-n_{q}[-\infty, y] \geq 2$. Defina $\alpha:=\max \{z \in(-\infty, y]$ : $p(z)=0\}$. Note que

$$
n_{p}[-\infty, y]=n_{p}[-\infty, \alpha] \quad \text { e } \quad n_{q}[-\infty, y] \geq n_{q}[-\infty, \alpha] .
$$

Logo, vale que $n_{p}[-\infty, \alpha]-n_{q}[-\infty, \alpha] \geq 2$. Suponha que

$$
n_{p}[-\infty, \alpha]-n_{q}[-\infty, \alpha] \geq 3 .
$$

Seja $\lambda \in[0,1]$. Note que $\lambda p^{\prime}+(1-\lambda) q^{\prime}=(\lambda p+(1-\lambda) q)^{\prime}$. Pela Proposição 4.2, temos que $(\lambda p+(1-\lambda) q)^{\prime}$ possui somente raízes reais. Logo, vale que $\lambda p^{\prime}+(1-\lambda) q^{\prime}$ também possui somente raízes reais. Pela Proposição 4.2 , temos que $n_{p^{\prime}}[-\infty, \alpha]=n_{p}[-\infty, \alpha]-1$ e $n_{q^{\prime}}[-\infty, \alpha] \leq n_{q}[-\infty, \alpha]$. Assim, vale que

$$
n_{p^{\prime}}[-\infty, \alpha]-n_{q^{\prime}}[-\infty, \alpha] \geq n_{p}[-\infty, \alpha]-1-n_{q}[-\infty, \alpha] \geq 2
$$

o que nos leva a uma contradição com a hipótese de indução.

Suponha agora que $n_{p}[-\infty, \alpha]-n_{q}[-\infty, \alpha]=2$. Seja $\beta \in(\alpha,+\infty)$ tal que $p(y) \neq 0$ e $q(y) \neq 0$ para todo $y \in(\alpha, \beta]$. Note que

$$
n_{p}[-\infty, \alpha]=n_{p}[-\infty, \beta] \text { e } n_{q}[-\infty, \alpha]=n_{p}[-\infty, \beta],
$$

e portanto $n_{p}[-\infty, \beta]-n_{q}[-\infty, \beta]=2$. Assim, vale que $n_{p}[-\infty, \beta]-n_{q}[-\infty, \beta]$ é par. Seja $\gamma$ um número real menor que todas as raízes de $p$ e de $q$. Dessa forma, como $p$ e $q$ são polinômios de grau $d$ e possuem coeficientes dominantes positivos, então

$$
p(\beta) q(\beta)>0 \quad \text { e } \quad p(\gamma) q(\gamma)>0 .
$$

Assim, pelo Lema 4.21, temos que $n_{p}[\gamma, \beta]=n_{q}[\gamma, \beta]$. Logo, como $n_{p}[\gamma, \beta]=n_{p}[-\infty, \beta]$ e $n_{q}[\gamma, \beta]=$ $n_{q}[-\infty, \beta]$, então vale que

$$
n_{p}[-\infty, \beta]=n_{q}[-\infty, \beta],
$$

o que nos leva a uma contradição.

Portanto, $n_{p}[-\infty, \alpha]-n_{q}[-\infty, \alpha] \leq 1$.

Teorema 4.23 ([8, Teorema 3.6]). Seja $Q \subseteq \mathbb{R}[x]$ um conjunto finito de polinômios de grau $d \geq 2$ que possuem somente raízes reais e coeficientes dominantes positivos. Então $Q$ possui um entrelaçador comum se e somente se $\sum_{q \in Q} \lambda_{q} q$ possui apenas raízes reais para todo $\lambda: Q \rightarrow \mathbb{R}_{+}$com $\sum_{q \in Q} \lambda_{q}=1$.

Demonstração. Suponha que $Q$ possui um entrelaçador comum. Seja $\lambda: Q \rightarrow \mathbb{R}_{+} \operatorname{com} \sum_{q \in Q} \lambda_{q}=1$. Seja $Q_{S}:=\operatorname{supp}(\lambda)$. Suponha que os polinômios do conjunto $Q_{S}$ possuem $k$ raízes em comum, ou seja, existem $a_{1}, a_{2}, \ldots, a_{k} \in \mathbb{R}$ tais que $\prod_{i \in[k]}\left(x-a_{i}\right)$ divide $q$ para todo $q \in Q_{S}$. Sejam $a_{1}, a_{2}, \ldots, a_{k}$ as raízes em comum dos polinômios de $Q_{S}$. Defina

$$
\bar{q}:=\frac{q}{\prod_{j \in[k]}\left(x-a_{j}\right)}
$$


para todo $q \in Q_{S}$. Note que não existe uma raiz comum entre todos os polinômios do conjunto $\left\{\bar{q}: q \in Q_{S}\right\}=: \bar{Q}_{S}$. Pelo Lema 4.7, temos que $\{\bar{p}, \bar{q}\}$ possui um entrelaçador comum para todos $\bar{p}, \bar{q} \in \bar{Q}_{S}$. Logo, pela Proposição 4.6, temos que $I_{\bar{p}}^{j} \cap I_{\bar{q}}^{j} \neq \varnothing$ para todo $j \in[d-k-1]$ e todos $\bar{p}, \bar{q} \in \bar{Q}_{S}$. Portanto, pelo Lema 2.6, vale que

$$
\bigcap_{\bar{q} \in \bar{Q}_{S}} I_{\bar{q}}^{j} \neq \varnothing
$$

para todo $j \in[d-k-1]$. Seja $b_{j} \in \bigcap_{\bar{q} \in \bar{Q}_{S}} I_{\bar{q}}^{j}$ para todo $j \in[d-k-1]$.

Note que, como os coeficientes dominantes dos polinômios de $Q_{S}$ são positivos, então os coeficientes dominantes dos polinômios de $\bar{Q}_{S}$ também são todos positivos. Logo, para todo $\bar{q} \in \bar{Q}_{S}$, vale que $\bar{q}\left(b_{d-k-j}\right) \geq 0$ para todo $j \in[d-k-1]$ par e $\bar{q}\left(b_{d-k-j}\right) \leq 0$ para todo $j \in[d-k-1]$ ímpar. Como os polinômios de $\bar{Q}_{S}$ não possuem raízes em comum, então, para todo $j \in[d-k-1]$, existe $\bar{q} \in \bar{Q}_{S}$ tal que $\bar{q}\left(b_{d-k-j}\right) \neq 0$. Assim, vale que

$$
\begin{array}{lr}
\left(\sum_{q \in Q_{S}} \lambda_{q} \bar{q}\right)\left(b_{d-k-j}\right)>0 & \forall j \in[d-k-1] \text { par, } \\
\left(\sum_{q \in Q_{S}} \lambda_{q} \bar{q}\right)\left(b_{d-k-j}\right)<0 & \forall j \in[d-k-1] \text { ímpar. }
\end{array}
$$

Portanto, pelo Teorema do Valor Intermediário, temos que $\sum_{q \in Q_{S}} \lambda_{q} \bar{q}$ possui $d-k$ raízes reais.

Note que

$$
\left(\prod_{j \in[k]}\left(x-a_{j}\right)\right)\left(\sum_{q \in Q_{S}} \lambda_{q} \bar{q}\right)=\sum_{q \in Q_{S}} \lambda_{q}\left(\bar{q} \prod_{j \in[k]}\left(x-a_{j}\right)\right)=\sum_{q \in Q_{S}} \lambda_{q} q=\sum_{q \in Q} \lambda_{q} q .
$$

Portanto, vale que $\sum_{q \in Q} \lambda_{q} q$ possui $d$ raízes reais. Isso conclui a prova de necessidade.

Sejam $p, q \in \mathbb{R}[x]$ polinômios de grau $d \geq 2$ com coeficientes dominantes de mesmo sinal. Suponha por contradição que $\{p, q\}$ não possui um entrelaçador comum e que $\lambda p+(1-\lambda) q$ possui somente raízes reais para todo $\lambda \in[0,1]$. Pela Proposição 4.6, temos que existe $j \in[d-1]$ tal que $I_{p}^{j} \cap I_{q}^{j}=\varnothing$. Seja

$$
j:=\max \left\{i \in[d-1]: I_{p}^{i} \cap I_{q}^{i}=\varnothing\right\} .
$$

Suponha, sem perda de generalidade, que $r_{j+1}(p)>r_{j+1}(q)$. Como $I_{p}^{j} \cap I_{q}^{j}=\varnothing$, então $r_{j+1}(q)<$ $r_{j}(p)$. Como $I_{p}^{i} \cap I_{q}^{i} \neq \varnothing$ para todo $i \in\{j+1, j+2, \ldots, d-1\}$, então $r_{j+2}(q) \geq r_{j+1}(p)$ se $j+2 \leq d$. Assim, se $j+2 \leq d$, então

$$
r_{j+1}(q)<r_{j}(p) \leq r_{j+1}(p) \leq r_{j+2}(q) .
$$

Seja $\gamma \in\left(r_{j+1}(q), r_{j}(p)\right)$. Note que $n_{p}[-\infty, \gamma] \leq j-1$ e $n_{q}[-\infty, \gamma]=j+1$, o que nos leva a uma contradição pelo Lema 4.22. Assim, se $Q$ não possui um entrelaçador, então, pela Proposição 4.6, temos que existem $p, q \in Q$ e $\ell \in[d-1]$ tais que $I_{p}^{\ell} \cap I_{q}^{\ell}=\varnothing$. Logo, pela Proposição 4.6, temos que $\{p, q\}$ não possuem um entrelaçador comum. Portanto, existem $\lambda_{p}, \lambda_{q} \in \mathbb{R}_{+} \operatorname{com} \lambda_{p}+\lambda_{q}=1$ tais que

$$
\lambda_{p} p+\lambda_{q} q
$$

não possui somente raízes reais. 
Considere a árvore de polinômios representada pela Figura 4.6, supondo que os nós que representam $p_{(0,0)}, p_{(0,1)}, p_{(0,2)}, p_{(1,0)}, p_{(1,1)}$ e $p_{(1,2)}$ são as folhas dessa árvore. Para mostrar que essa árvore de polinômios é entrelaçadora, basta mostrar que todas as combinações convexas de $p_{(0,0)}, p_{(0,1)}$ e $p_{(0,2)}$, de $p_{(1,0)}, p_{(1,1)}$ e $p_{(1,2)}$ e de $p_{(0)}$ e $p_{(1)}$ possuem somente raízes reais. Dessa forma, basta mostrar que combinações convexas de $p_{(0,0)}, p_{(0,1)}, p_{(0,2)}, p_{(1,0)}, p_{(1,1)}$ e $p_{(1,2)}$, com certos suportes, possuem somente raízes reais:

$$
\lambda_{(0,0)} p_{(0,0)}+\lambda_{(0,1)} p_{(0,1)}+\lambda_{(0,2)} p_{(0,2)}+\lambda_{(1,0)} p_{(1,0)}+\lambda_{(1,1)} p_{(1,1)}+\lambda_{(1,2)} p_{(1,2)}
$$

possui somente raízes reais para todos $\lambda_{(0,0)}, \lambda_{(0,1)}, \lambda_{(0,2)}, \lambda_{(1,0)}, \lambda_{(1,1)}, \lambda_{(1,2)} \in \mathbb{R}_{+}$com

$$
\begin{gathered}
\bullet \lambda_{(0,0)}=\lambda_{(0,1)}=\lambda_{(0,2)}=0, \\
\bullet \lambda_{(1,0)}=\lambda_{(1,1)}=\lambda_{(1,2)}=0 \quad \text { ou } \\
\bullet \lambda_{(0,0)}=\lambda_{(0,1)}=\lambda_{(0,2)} \quad \text { e } \quad \lambda_{(1,0)}=\lambda_{(1,1)}=\lambda_{(1,2)},
\end{gathered}
$$

tais que $\sum_{i \in\{0,1\}} \sum_{j \in\{0,1,2\}} \lambda_{(i, j)}=1$.

Essa caracterização ainda não torna simples a identificação de árvores entrelaçadoras de polinômios. Por esse motivo, utilizamos polinômios estáveis como ferramenta para mostrar que determinadas combinações convexas dos polinômios de certa família possuem somente raízes reais. 


\section{Capítulo 5}

\section{Polinômios estáveis}

Neste capítulo, definiremos polinômios estáveis, que são polinômios de coeficientes complexos, onde a parte imaginária de cada uma de suas raízes é no máximo zero em pelo menos uma coordenada.

Definição 5.1. Seja $p \in \mathbb{C}\left[x_{1}, \ldots, x_{m}\right]$. Dizemos que $p$ é estável se, para todo $x \in \mathbb{C}^{m}$ tal que $p(x)=0$, existe $j \in[m]$ tal que $\operatorname{Im}\left(x_{j}\right) \leq 0$.

Seja $p \in \mathbb{C}\left[x_{1}, x_{2}, x_{3}\right]$ um polinômio estável multivariado definido como

$$
p\left(x_{1}, x_{2}, x_{3}\right):=x_{1}^{2} x_{2} x_{3}+\boldsymbol{i} x_{1}^{2} x_{2}-x_{2} x_{3}-\boldsymbol{i} x_{2} .
$$

Note que $p$ pode ser escrito da seguinte forma: $p\left(x_{1}, x_{2}, x_{3}\right)=\left(x_{1}^{2}-1\right)\left(x_{2}\right)\left(x_{3}+\boldsymbol{i}\right)$. Assim, é fácil ver que $r_{1}:=(-\boldsymbol{i},-\boldsymbol{i},-\boldsymbol{i}), r_{2}:=(-1, \boldsymbol{i}+1,-\boldsymbol{i}+2), r_{3}:=(\boldsymbol{i}-1,0,2 \boldsymbol{i}-1)$ e $r_{4}:=(1,3+\boldsymbol{i}, 4+\boldsymbol{i})$ são algumas das raízes de $p$. Utilizamos a Figura 5.1 para ilustrar o comportamento das raízes desse polinômio.

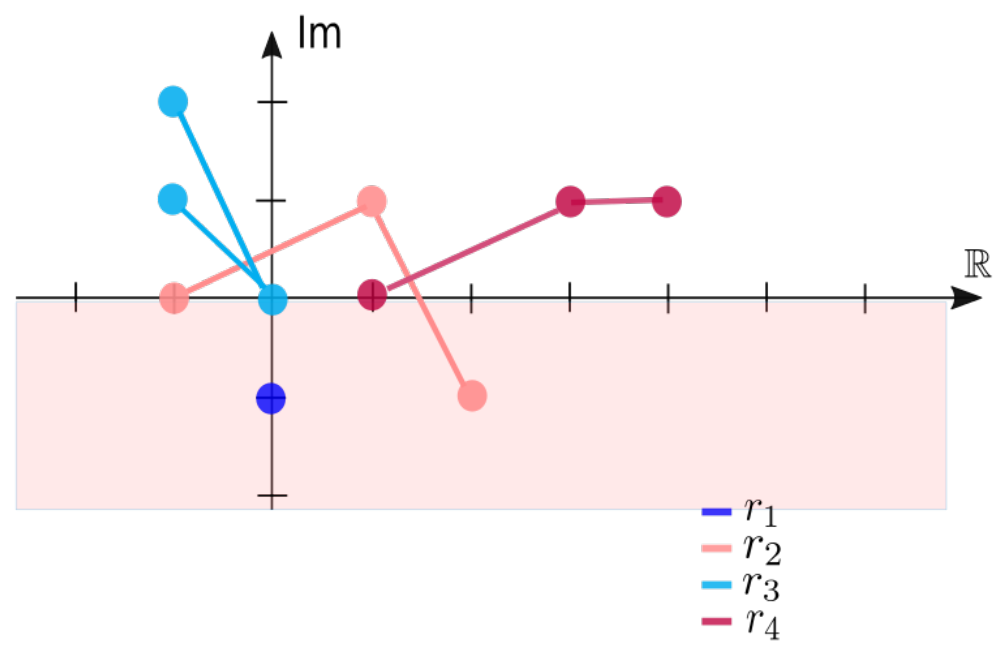

Figura 5.1: Representação das raízes do polinômio $p$, definido em (5.1), onde cada uma de suas raízes é representada por uma cor e cada ponto representa o valor associado a alguma coordenada.

Note também que a parte imaginária de cada uma das raízes de $p$ apresentadas é no máximo zero em pelo menos uma das coordenadas. Apesar de o polinômio $p$ possuir infinitas raízes, mostramos apenas algumas delas com intuito de ilustrar as raízes de polinômios estáveis. 
Definiremos também polinômios reais estáveis, que são polinômios estáveis com coeficientes reais.

Definição 5.2. Seja $p \in \mathbb{C}\left[x_{1}, \ldots, x_{m}\right]$. Dizemos que $p$ é um polinômio real estável se $p$ é estável e se $p \in \mathbb{R}\left[x_{1}, \ldots, x_{m}\right]$.

Esses polinômios são utilizados como uma generalização de polinômios que possuem somente raízes reais, pois qualquer polinômio real estável monovariado possui somente raízes reais, como mostrado abaixo.

Proposição 5.3. Seja $p \in \mathbb{R}[x]$ um polinômio monovariado. Então $p$ é real estável se e somente se $p$ possui somente raízes reais.

Demonstração. Suponha que $p$ é real estável. Sejam $A \subseteq \mathbb{N}$ finito e $c: A \rightarrow \mathbb{R}$ uma função tais que $p=\sum_{a \in A} c_{a} x^{a}$. Seja $y \in \mathbb{C}$ tal que $p(y)=0$. Pelas propriedades de conjugação, temos que

$$
0=\overline{p(y)}=\overline{\sum_{a \in A} c_{a} y^{a}}=\sum_{a \in A} \overline{c_{a} y^{a}}=\sum_{a \in A} c_{a}(\bar{y})^{a}=p(\bar{y}) .
$$

Assim, vale que $\bar{y}$ também é uma raiz de $p$. Sejam $a, b \in \mathbb{R}$ tais que $y=a+b \boldsymbol{i}$. Como $p$ é real estável, então $b \leq 0$. Além disso, como $p(\bar{y})=0$, então $b \geq 0$. Portanto, vale que $y \in \mathbb{R}$.

Suponha agora que $p$ possui somente raízes reais. Pela Definição 5.1, é fácil ver que $p$ é estável.

Apresentaremos uma caracterização para cada uma dessas definições, exemplos de polinômios estáveis e reais estáveis e exemplos de operações em polinômios que preservam estabilidade e real estabilidade.

Seja $q \in \mathbb{R}\left[x_{1}, x_{2}, x_{3}\right]$ o polinômio real estável multivariado definido como

$$
q\left(x_{1}, x_{2}, x_{3}\right):=x_{1}^{2} x_{2}+x_{1}^{2} x_{2} x_{3} .
$$

Note que o polinômio $q$ pode ser escrito da seguinte maneira: $q\left(x_{1}, x_{2}, x_{3}\right)=x_{1}^{2} x_{2}\left(x_{3}+1\right)$. Dessa forma, é fácil ver que $r_{1}:=(\boldsymbol{i}, \boldsymbol{i}+1,-1), r_{2}:=(\boldsymbol{i}-1,2 \boldsymbol{i}-2,-1), r_{3}:=(0, \boldsymbol{i}+3,-\boldsymbol{i}+4)$ e $r_{4}:=$ $(-\boldsymbol{i}-1,0,-\boldsymbol{i}+3)$ são algumas das raízes de $q$. Ilustramos o comportamento das raízes desse polinômio real estável na Figura 5.2.

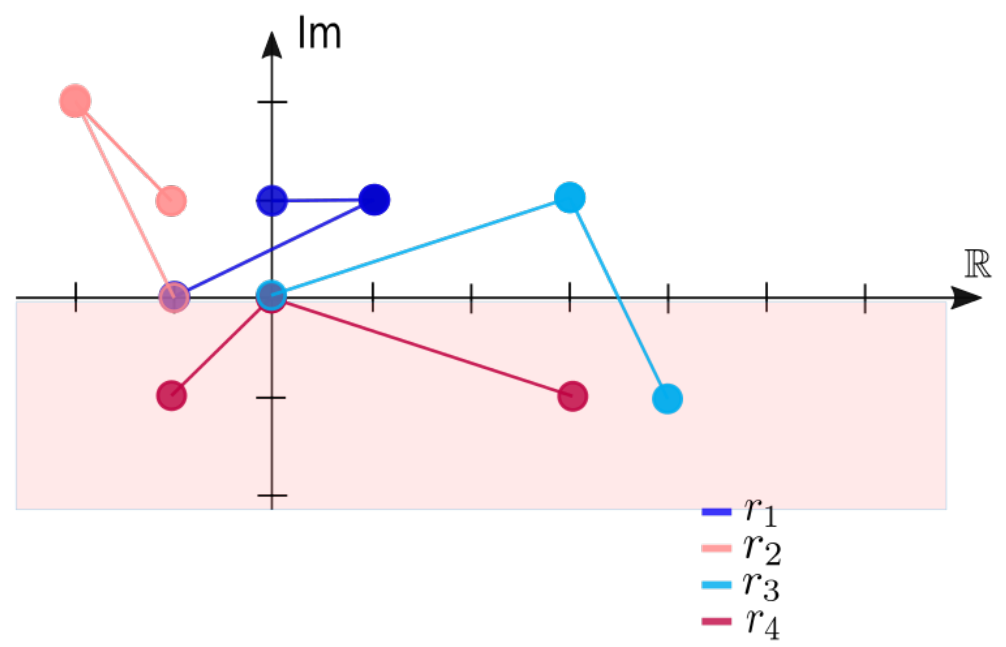

Figura 5.2: Representação das raízes do polinômio q, definido em (5.2), onde cada uma de suas raízes é representada por uma cor e cada ponto representa o valor associado a alguma coordenada. 
Assim como as raízes do polinômio $p\left(x_{1}, x_{2}, x_{3}\right)=x_{1}^{2} x_{2} x_{3}+\boldsymbol{i} x_{1}^{2} x_{2}-x_{2} x_{3}-\boldsymbol{i} x_{2}$, a parte imaginária de cada uma das raízes de $q$ apresentadas também é no máximo zero em pelo menos uma das coordenadas. Mostramos apenas algumas das infinitas raízes de $q$ para ilustrar o comportamento das raízes de polinômios reais estáveis.

Mostraremos que $p$ e $q$ são realmente estável e real estável, respectivamente, utilizando caracterizações de polinômios estáveis e reais estáveis que serão apresentadas na próxima seção.

\subsection{Caracterizações de estabilidade}

Se um polinômio é monovariado, então é simples ter conhecimento de todas as suas raízes, pois o número de raízes de um polinômio monovariado é finito e, além disso, é possível decompor esse polinômio em fatores de primeiro grau, o que nos leva diretamente a todas as suas raízes. Dessa forma, é fácil identificar um polinômio estável ou real estável caso este seja monovariado. Porém, caso o polinômio seja multivariado, não é simples identificar se ele é estável, pois polinômios multivariados podem possuir infinitas raízes e, para identificar se são estáveis, teríamos que ter conhecimento de todas as infinitas raízes desse polinômio.

As caracterizações apresentadas abaixo simplificam o problema de identificar polinômios multivariados estáveis ou reais estáveis para um problema de identificar polinômios monovariados estáveis ou reais estáveis. Assim, para saber se um polinômio é ou não estável, basta transformá-lo num polinômio monovariado, de acordo com os resultados abaixo, e verificar a sua estabilidade.

Teorema 5.4. Seja $p \in \mathbb{C}\left[x_{1}, \ldots, x_{m}\right]$. Então $p$ é estável se e somente se para todo $d \in \mathbb{R}_{++}^{m}$ e todo $y \in \mathbb{R}^{m}$, o polinômio monovariado definido por

$$
t \mapsto p(y+t d)
$$

é estável.

Demonstração. Suponha primeiramente que $p$ não é estável. Portanto, existe $x \in \mathbb{C}^{m}$ tal que $p(x)=0$ e $\operatorname{Im}(x)>0$. Sejam $a \in \mathbb{R}^{m}$ e $b \in \mathbb{R}_{++}^{m}$ tais que $x=a+\boldsymbol{i} b$. Como $p(a+\boldsymbol{i} b)=0$, então $\boldsymbol{i}$ é uma das raízes do polinômio definido por $t \mapsto p(a+t b)$ e, portanto, este não é estável.

Por outro lado, suponha que existem $d \in \mathbb{R}_{++}^{m}$ e $y \in \mathbb{R}^{m}$ tais que o polinômio definido por $t \mapsto p(y+t d)$ não é estável. Logo, existe $t \in \mathbb{C}$ tal que $\operatorname{Im}(t)>0$ e $p(y+t d)=0$. Note que $\operatorname{Im}(y+t d)=\operatorname{Im}(t) d>0$. Portanto $p$ não é estável.

Considere o polinômio $p\left(x_{1}, x_{2}, x_{3}\right)=x_{1}^{2} x_{2} x_{3}+\boldsymbol{i} x_{1}^{2} x_{2}-x_{2} x_{3}-\boldsymbol{i} x_{2}$ definido anteriormente. Mostraremos agora que $p$ realmente é um polinômio estável utilizando o resultado apresentado acima. Pelo Teorema 5.4, para mostrar que $p$ é estável, basta mostrar que, para todo $d \in \mathbb{R}_{++}^{n} \mathrm{e}$ todo $y \in \mathbb{R}^{n}$, o polinômio $p_{d, y} \in \mathbb{C}[t]$ definido como $p_{d, y}(t):=E_{x=y+t d}(p)$ é estável. Note que $p_{d, y}$ pode ser escrito da seguinte maneira:

$$
p_{d, y}(t)=\left(y_{1}+t d_{1}+1\right)\left(y_{1}+t d_{1}-1\right)\left(y_{2}+t d_{2}\right)\left(y_{3}+t d_{3}+\boldsymbol{i}\right) .
$$

Assim, é fácil ver que as raízes de $p_{d, y}$ são $-\left(y_{1}+1\right) / d_{1},\left(-y_{1}+1\right) / d_{1},-y_{2} / d_{2}$ e $-\left(y_{3}+i\right) / d_{3}$. Como a parte imaginária de todas as raízes de $p_{d, y}$ é no máximo zero, então o polinômio $p_{d, y}$ é estável, e, portanto, podemos concluir que $p$ é estável. 
Teorema 5.5. Seja $p \in \mathbb{C}\left[x_{1}, \ldots, x_{m}\right]$. Então $p$ é real estável se e somente se, para todo $d \in \mathbb{R}_{++}^{m}$ e todo $y \in \mathbb{R}^{m}$, o polinômio monovariado definido por

$$
t \mapsto p(y+t d)
$$

possui somente raízes reais.

Demonstração. Suponha que $p$ não é real estável. Assim, vale que $p$ não é estável ou $p \notin \mathbb{R}\left[x_{1}, \ldots, x_{m}\right]$. Se $p$ não é estável, pelo Teorema 5.4, temos que existem $d \in \mathbb{R}_{++}^{m}$ e $y \in \mathbb{R}^{m}$ tais que o polinômio definido por $t \mapsto p(y+t d)$ também não é estável, e portanto não possui somente raízes reais. Suponha que $p \notin \mathbb{R}\left[x_{1}, \ldots, x_{m}\right]$. Logo, existem $a \in \mathbb{R}^{m}$ e $b \in \mathbb{R}_{++}^{m}$ tais que o polinômio monovariado $q \in \mathbb{C}[t]$ definido como $q(t)=p(a+t b)$ não pertence a $\mathbb{R}[t]$. Dessa forma, existe $y \in \mathbb{C} \backslash \mathbb{R}$ tal que $q(y)=0$ e, portanto, o polinômio $q$ não possui somente raízes reais.

Por outro lado, suponha que existem $d \in \mathbb{R}_{++}^{m}$ e $y \in \mathbb{R}^{m}$ tais que o polinômio $q \in \mathbb{C}[t]$ definido por $q(t)=p(y+t d)$ não possui somente raízes reais. Se existe $t \in \mathbb{C}$ tal que $\operatorname{Im}(t)>0$ e $q(t)=0$, então, pelo Teorema 5.4, o polinômio $p$ não é estável. Suponha então que $\operatorname{Im}(t) \leq 0$ para todo $t \in \mathbb{C}$ tal que $q(t)=0$. Logo, como $q$ não possui somente raízes reais, existe $t \in \mathbb{C}$ tal que $\operatorname{Im}(t)<0$ e $q(t)=0$. Assim, como $q$ é um polinômio com uma única variável, então $q$ possui pelo menos um coeficiente complexo não-real. Portanto, o polinômio $p$ também possui pelo menos um coeficiente complexo não-real, já que $d, y \in \mathbb{R}^{m}$.

Considere o polinômio $q\left(x_{1}, x_{2}, x_{3}\right)=x_{1}^{2} x_{2}+x_{1}^{2} x_{2} x_{3}$ definido anteriormente. Pelo resultado apresentado acima, o polinômio $q$ é real estável se e somente se, para todo $d \in \mathbb{R}_{++}^{3}$ e todo $y \in \mathbb{R}^{3}$, o polinômio $q_{d, y} \in \mathbb{C}[t]$ definido como $q_{d, y}:=E_{x=y+t d}(q)$ possui somente raízes reais. Sejam $d \in \mathbb{R}_{++}^{3}$ e $y \in \mathbb{R}^{3}$. Note que $q_{d, y}$ pode ser escrito da seguinte forma:

$$
q_{d, y}(t)=\left(y_{1}+t d_{1}\right)^{2}\left(y_{2}+t d_{2}\right)\left(1+y_{3}+t d_{3}\right) .
$$

Assim, é fácil ver que as raízes de $q_{d, y}$ são $-y_{1} / d_{1},-y_{1} / d_{1},-y_{2} / d_{2}$ e $-\left(y_{3}+1\right) / d_{3}$ e, portanto, as raízes de $q_{d, y}$ são todas reais. Dessa forma, temos agora uma prova de que o polinômio $q$ é real estável sem a necessidade de verificar todas as suas raízes.

\subsection{Preservadores de estabilidade}

Nesta seção, mostraremos algumas operações em polinômios que preservam a estabilidade e a estabilidade real. Assim, ao aplicar alguma dessas operações em polinômios estáveis, obtemos outro polinômio estável, e o mesmo vale para polinômios reais estáveis. A vantagem de utilizar essas operações é que, a partir de um polinômio estável cuja estabilidade é facilmente verificada, podemos obter outros polinômios estáveis ou reais estáveis que não são facilmente identificados. Essas operações irão nos auxiliar a mostrar alguns exemplos de polinômios reais estáveis na próxima seção que, por sua vez, serão utilizados para mostrar que algumas famílias de polinômios formam árvores entrelaçadoras de polinômios. Seguem algumas operações básicas de especificação de indeterminadas que preservam a estabilidade.

Teorema 5.6. Sejam $p \in \mathbb{C}\left[x_{1}, \ldots, x_{m}\right]$ um polinômio estável e $i, j \in[m]$. Tome $X:=\left\{x_{1}, \ldots, x_{m}\right\}$.

a) Seja $\pi: X \rightarrow X$ uma permutação de $X$. Então o polinômio $E_{\pi}(p)$ é estável.

b) Seja $c \in \mathbb{R}$. Então $E_{x_{i}=c+x_{i}}(p)$ é estável. 
c) Então $E_{x_{i}=x_{j}}$ é estável.

d) Seja $c \in\{y \in \mathbb{C}: \operatorname{Im}(y) \geq 0\}$. Então $E_{x_{i}=c}(p)$ é estável ou $E_{x_{i}=c}(p)=0$.

e) Seja $c \in \mathbb{R}_{+}$. Então $E_{x_{i}=c x_{i}}(p)$ é estável ou $E_{x_{i}=c x_{i}}(p)=0$.

Demonstração. a) Seja $y \in \mathbb{C}^{m}$ uma raiz de $E_{\pi}(p)$. Logo, vale que $\left(y_{\pi^{-1}(1)}, y_{\pi^{-1}(2)}, \ldots, y_{\pi^{-1}(m)}\right)$ é uma raiz de $p$.

Como $p$ é estável, então existe $j \in[m]$ tal que $\operatorname{Im}\left(y_{\pi^{-1}(j)}\right) \leq 0$. Portanto, existe $k \in[m]$ tal que $\operatorname{Im}\left(y_{k}\right) \leq 0$.

b) Seja $y \in \mathbb{C}^{m}$ uma raiz de $E_{x_{i}=c+x_{i}}(p)$. Note que $y+c e_{i}$ é raiz de $p$.

Como $p$ é estável, então $\operatorname{Im}\left(y_{i}+c\right) \leq 0$ ou existe $j \in[m] \backslash\{i\}$ tal que $\operatorname{Im}\left(y_{j}\right) \leq 0 . \operatorname{Se} \operatorname{Im}\left(y_{i}+c\right) \leq 0$, então $\operatorname{Im}\left(y_{i}\right) \leq 0$. Portanto, existe $j \in[m]$ tal que $\operatorname{Im}\left(y_{j}\right) \leq 0$.

c) Seja $y \in \mathbb{C}^{[m] \backslash\{i\}}$ uma raiz de $E_{x_{i}=x_{j}}(p)$. Assim, $\left(y_{1}, \ldots, y_{i-1}, y_{j}, y_{i+1}, \ldots, y_{m}\right)$ é raiz de $p$.

Como $p$ é estável, então existe $k \in[m] \backslash\{i\}$ tal que $\operatorname{Im}\left(y_{k}\right) \leq 0$.

d) Suponha que $E_{x_{i}=c}(p) \neq 0$. Suponha primeiro que $c \notin \mathbb{R}$. Seja $y \in \mathbb{C}^{[m] \backslash\{i\}}$ uma raiz de $E_{x_{i}=c}(p)$. Defina $y^{\prime} \in \mathbb{C}^{m}$ como

$$
y_{j}^{\prime}:= \begin{cases}c, & \text { se } j=i \\ y_{j}, & \text { se } j \in[m] \backslash\{i\} .\end{cases}
$$

Note que $y^{\prime}$ é raiz de $p$. Como $p$ é estável, então existe $j \in[m]$ tal que $\operatorname{Im}\left(y_{j}^{\prime}\right) \leq 0$. Como $\operatorname{Im}\left(y_{i}^{\prime}\right)=\operatorname{Im}(c)>0$, então existe $j \in[m] \backslash\{i\}$ tal que $\operatorname{Im}\left(y_{j}\right)=\operatorname{Im}\left(y_{j}^{\prime}\right) \leq 0$.

Por outro lado, suponha que $c \in \mathbb{R}$. Suponha também, por contradição, que $E_{x_{i}=c}(p)$ não é estável. Como $E_{x_{i}=c}(p)$ não é estável, então existe uma raiz $y \in \mathbb{C}^{[m] \backslash\{i\}}$ de $E_{x_{i}=c}(p)$ tal que $\operatorname{Im}\left(y_{j}\right)>0$ para todo $j \in[m] \backslash\{i\}$. Seja $\ell \in[m] \backslash\{i\}$ e seja $f_{k}:\left\{x_{j}: j \in[m] \backslash\{\ell\}\right\} \rightarrow\{z \in \mathbb{C}:$ $\operatorname{Im}(z)>0\}$ definida como

$$
f_{k}\left(x_{j}\right)= \begin{cases}c+i / k & \text { se } j=i \\ y_{j} & \text { se } j \in[m] \backslash\{i, \ell\}\end{cases}
$$

para todo $k \in \mathbb{N} \backslash\{0\}$ e todo $j \in[m] \backslash\{\ell\}$. Defina $f_{0}:\left\{x_{j}: j \in[m] \backslash\{\ell\}\right\} \rightarrow\{z \in \mathbb{C}: \operatorname{Im}(z) \geq 0\}$ definida como

$$
f_{0}\left(x_{j}\right)= \begin{cases}c & \text { se } j=i \\ y_{j} & \text { se } j \in[m] \backslash\{i, \ell\} .\end{cases}
$$

para todo $j \in[m] \backslash\{\ell\}$. Pelo primeiro caso, temos que $E_{f_{k}}$ preserva estabilidade para todo $k \in \mathbb{N} \backslash\{0\}$. Assim, como $p$ é estável, então o polinômio $E_{f_{k}}(p)$ é estável para todo $k \in \mathbb{N} \backslash\{0\}$. Note também que $\left(E_{f_{k}}(p)\right)_{k \in \mathbb{N}}$ satisfaz as condições do Teorema 4.16. Logo, para todo $X \subseteq \mathbb{C} \backslash \mathbb{R}$ compacto, vale que $\left(E_{f_{k}}(p)\right)_{k \in \mathbb{N} \backslash\{0\}}$ converge uniformemente para $E_{f_{0}}(p)$. Como $E_{f_{k}}(p)$ é estável, então $\left(E_{f_{k}}(p)\right)(w) \neq 0$ para todo $k \in \mathbb{N} \backslash\{0\}$ e todo $w \in\{z \in \mathbb{C}: \operatorname{Im}(z)>0\}$, o que implica, pelo Corolário 4.15 , que $\left(E_{f_{0}}(p)\right)(w) \neq 0$ para todo $w \in\{z \in \mathbb{C}: \operatorname{Im}(z)>0\}$. Logo, vale que $\left(E_{f_{0}}(p)\right)\left(y_{\ell}\right) \neq 0$ e, portanto, $y$ não é uma raiz de $E_{x_{i}=c}(p)$, o que nos leva a uma contradição.

e) Suponha que $E_{x_{i}=c x_{i}}(p) \neq 0$. Se $c=0$, então $E_{x_{i}=c x_{i}}(p)$ é estável pelo Item d).

Suponha que $c>0$. Seja $y \in \mathbb{C}^{m}$ uma raiz de $E_{x_{i}=c x_{i}}(p)$. Note que $\left(y_{1}, \ldots, y_{i-1}, c y_{i}, y_{i+1}, \ldots, y_{m}\right)$ é raiz de $p$.

Como $p$ é estável, então $\operatorname{Im}\left(c y_{i}\right) \leq 0$ ou existe $j \in[m] \backslash\{i\}$ tal que $\operatorname{Im}\left(y_{j}\right) \leq 0 . \operatorname{Se} \operatorname{Im}\left(c y_{i}\right) \leq 0$, então $\operatorname{Im}\left(y_{i}\right) \leq 0$, o que implica que existe $j \in[m]$ tal que $\operatorname{Im}\left(y_{j}\right) \leq 0$. 
A prova desses resultados consiste em analisar as raízes dos polinômios resultantes de cada operação e compará-las com as raízes do polinômio original.

Considere o polinômio estável $p\left(x_{1}, x_{2}, x_{3}\right):=x_{1}^{2} x_{2} x_{3}+\boldsymbol{i} x_{1}^{2} x_{2}-x_{2} x_{3}-\boldsymbol{i} x_{2}$ definido anteriormente. Pelo Teorema 5.6, temos que os polinômios em $\mathbb{C}\left[x_{1}, x_{2}, x_{3}\right]$ definidos a seguir são estáveis:

$$
\begin{aligned}
E_{x_{1}=x_{3}, x_{3}=x_{1}}(p) & =x_{3}^{2} x_{2} x_{1}+\boldsymbol{i} x_{3}^{2} x_{2}-x_{2} x_{1}-\boldsymbol{i} x_{2}, \\
E_{x_{3}=5+x_{3}}(p) & =5 x_{1}^{2} x_{2}+x_{1}^{2} x_{2} x_{3}+\boldsymbol{i} x_{1}^{2} x_{2}-5 x_{2}-x_{2} x_{3}-\boldsymbol{i} x_{2}, \\
E_{x_{2}=x_{1}}(p) & =x_{1}^{3} x_{3}+\boldsymbol{i} x_{1}^{3}-x_{1} x_{3}-\boldsymbol{i} x_{1}, \\
E_{x_{2}=1}(p) & =x_{1}^{2} x_{3}+\boldsymbol{i} x_{1}^{2}-x_{3}-\boldsymbol{i}, \\
E_{x_{1}=2 x_{1}}(p) & =4 x_{1}^{2} x_{2} x_{3}+\boldsymbol{i} 4 x_{1}^{2} x_{2}-x_{2} x_{3}-\boldsymbol{i} x_{2} .
\end{aligned}
$$

É fácil verificar que todos os polinômios citados acima são estáveis utilizando a caracterização de polinômios estáveis apresentada na seção anterior. Também podemos aplicar, em um polinômio, várias combinações de operações que preservam estabilidade e, assim, obter polinômios estáveis variados. Além de preservarem a estabilidade, as operações referentes ao Teorema 5.6 também preservam a estabilidade real. Esse resultado segue, de forma direta, do resultado anterior.

Corolário 5.7. Seja $p \in \mathbb{C}\left[x_{1}, x_{2}, \ldots, x_{m}\right]$ um polinômio real estável e seja $i, j \in[m]$. Defina $X:=\left\{x_{1}, \ldots, x_{m}\right\}$.

a) Seja $\pi: X \rightarrow X$ uma permutação de $X$. Então o polinômio $E_{\pi}(p)$ é real estável.

b) Seja $c \in \mathbb{R}$. Então $E_{x_{i}=c+x_{i}}(p)$ é real estável.

c) Então $E_{x_{i}=x_{j}}$ é real estável.

d) Seja $c \in \mathbb{R}$. Então $E_{x_{i}=c}(p)$ é real estável ou $E_{x_{i}=c}(p)=0$.

e) Seja $c \in \mathbb{R}_{+}$. Então $E_{x_{i}=c x_{i}}(p)$ é real estável ou $E_{x_{i}=c x_{i}}(p)=0$.

Demonstração. Segue do Teorema 5.6.

Para ilustrar esse resultado, considere o polinômio real estável $q\left(x_{1}, x_{2}, x_{3}\right):=x_{1}^{2} x_{2}+x_{1}^{2} x_{2} x_{3}$ definido anteriormente. Pelo Corolário 5.7, temos que os polinômios

$$
\begin{aligned}
E_{x_{1}=x_{3}, x_{3}=x_{1}}(q) & =x_{3}^{2} x_{2}+x_{3}^{2} x_{2} x_{1}, \\
E_{x_{3}=5+x_{3}}(q) & =x_{1}^{2} x_{2}+5 x_{1}^{2} x_{2}+x_{1}^{2} x_{2} x_{3}, \\
E_{x_{2}=x_{1}}(q) & =x_{1}^{3}+x_{1}^{3} x_{3}, \\
E_{x_{2}=1}(q) & =x_{1}^{2}+x_{1}^{2} x_{3}, \\
E_{x_{1}=2 x_{1}}(q) & =4 x_{1}^{2} x_{2}+4 x_{1}^{2} x_{2} x_{3}
\end{aligned}
$$

são reais estáveis. Note que é fácil verificar que esses polinômios são reais estáveis pela caracterização de polinômios reais estáveis apresentada anteriormente.

A seguir, apresentaremos uma caracterização de certas operações que preservam a estabilidade real. Essa caracterização simplifica o problema de identificar operações que preservam a estabilidade real para um problema de identificar polinômios reais estáveis. Dessa forma, para saber se uma certa operação preserva real estabilidade, basta verificar se o polinômio associado a essa operação é real estável. Essa caracterização se deve a Borcea e Brändén [5] e será utilizada como caixa-preta neste texto. Antes de enunciá-la, apresentaremos algumas notações que serão utilizadas pela mesma. 
Definição 5.8. Sejam $x \in \mathbb{C}^{m}$ e $\beta \in \mathbb{N}^{m}$. Defina

$$
x^{\beta}:=\prod_{i \in[m]} x_{i}^{\beta_{i}} .
$$

Definição 5.9. Seja $\beta \in \mathbb{N}^{m}$. Defina a função $\partial^{\beta}: \mathbb{R}\left[x_{1}, x_{2}, \ldots, x_{m}\right] \rightarrow \mathbb{R}\left[x_{1}, x_{2}, \ldots, x_{m}\right]$ como

$$
\partial^{\beta}:=\prod_{i \in[m]}\left(\frac{\partial}{\partial x_{i}}\right)^{\beta_{i}} .
$$

Teorema 5.10 ([5, Teorema 1.3]). Sejam $A, B \subseteq \mathbb{N}^{m}$ finitos e $c_{\alpha, \beta} \in \mathbb{R}$ para todo $\alpha \in A$ e todo $\beta \in B$. Seja $T: \mathbb{C}\left[x_{1}, \ldots, x_{m}\right] \rightarrow \mathbb{C}\left[x_{1}, \ldots, x_{m}\right]$ tal que

$$
T(p)=\sum_{\substack{\alpha \in A \\ \beta \in B}} c_{\alpha, \beta} x^{\alpha} \partial^{\beta}(p)
$$

para todo $p \in \mathbb{C}\left[x_{1}, \ldots, x_{m}\right]$. Então $T$ preserva a estabilidade real se e somente se

$$
F_{T}(x, z):=\sum_{\substack{\alpha \in A \\ \beta \in B}} c_{\alpha, \beta} x^{\alpha} z^{\beta} \in \mathbb{R}\left[x_{1}, \ldots, x_{m}, z_{1}, \ldots, z_{m}\right]
$$

é real estável.

Na Seção 5.4, mostraremos alguns exemplos de árvores entrelaçadoras de polinômios, que serão utilizados para provar a existência de famílias infinitas de grafos de Ramanujan e para provar uma solução do Problema de Kadison-Singer. Para provar que esses exemplos são árvores entrelaçadoras de polinômios, utilizamos operações que preservam a estabilidade real. Utilizaremos o resultado de Borcea e Brändén para mostrar que essas operações preservam a estabilidade real. Para isso, mostraremos a seguir que os polinômios associados a essas operações são reais estáveis.

Proposição 5.11. Sejam $c_{1}, c_{2} \in \mathbb{R}_{+}$. Então, o polinômio $p \in \mathbb{R}\left[x_{1}, x_{2}\right]$ definido por $p\left(x_{1}, x_{2}\right):=$ $1-c_{1} x_{1}-c_{2} x_{2}$ é real estável.

Demonstração. Seja $q \in \mathbb{R}\left[x_{1}, x_{2}\right]$ definido por $q\left(x_{1}, x_{2}\right):=1-x_{1}-x_{2}$. Seja $y \in \mathbb{C}^{2}$ uma raiz de q. Note que $y_{1}+y_{2}=1$, o que implica que $\operatorname{Im}\left(y_{1}\right)+\operatorname{Im}\left(y_{2}\right)=0$. Logo, vale que $\operatorname{Im}\left(y_{1}\right) \leq 0$ ou $\operatorname{Im}\left(y_{2}\right) \leq 0$, o que implica que $q$ é real estável. Portanto, como $p=E_{x_{1}=c_{1} x_{1}, x_{2}=c_{2} x_{2}}(q)$, então $p$ é real estável pelo Corolário 5.7(e).

Proposição 5.12. Sejam $c_{1}, c_{2} \in \mathbb{R}_{+}$. Então, o polinômio $p \in \mathbb{R}\left[x_{1}, x_{2}\right]$ definido por $p\left(x_{1}, x_{2}\right):=$ $1+c_{1} x_{1}+c_{2} x_{2}$ é real estável.

Demonstração. Seja $q \in \mathbb{R}\left[x_{1}, x_{2}\right]$ definido por $q\left(x_{1}, x_{2}\right):=1+x_{1}+x_{2}$. Seja $y \in \mathbb{C}^{2}$ uma raiz de q. Note que $y_{1}+y_{2}=-1$, o que implica que $\operatorname{Im}\left(y_{1}\right)+\operatorname{Im}\left(y_{2}\right)=0$. Assim, vale que $\operatorname{Im}\left(y_{1}\right) \leq 0$ ou $\operatorname{Im}\left(y_{2}\right) \leq 0$ e, portanto, $q$ é real estável. Logo, como $p=E_{x_{1}=c_{1} x_{1}, x_{2}=c_{2} x_{2}}(q)$, então $p$ é real estável pelo Corolário 5.7(e).

Apresentaremos agora alguns exemplos de preservadores de real estabilidade, utilizando o Teorema 5.10 e os exemplos de polinômios reais estáveis mostrados acima.

Corolário 5.13. Sejam $i, j \in[m]$. Sejam $c_{i}, c_{j} \in \mathbb{R}_{+}$e $T: \mathbb{R}\left[x_{1}, \ldots, x_{m}\right] \rightarrow \mathbb{R}\left[x_{1}, \ldots, x_{m}\right]$ definido por $T=1+c_{i} \partial_{x_{i}}+c_{j} \partial_{x_{j}}$. Então $T$ preserva estabilidade real. 
Demonstração. Sejam $x_{1}, \ldots, x_{m}, z_{1}, \ldots, z_{m}$ indeterminadas algebricamente independentes. Defina $A, B \subseteq \mathbb{N}^{m}$ por $A:=\{0\}$ e $B:=\left\{0, e_{i}, e_{j}\right\}$. Seja $c: B \rightarrow \mathbb{R}$ tal que $c(0)=1, c\left(e_{i}\right)=c_{i}$ e $c\left(e_{j}\right)=c_{j}$. Note que

$$
\sum_{\substack{\alpha \in A \\ \beta \in B}} c_{\beta} x^{\alpha} z^{\beta}=1+c_{i} z_{i}+c_{j} z_{j} .
$$

Pela Proposição 5.12, temos que $1+c_{i} z_{i}+c_{j} z_{j}$ é um polinômio real estável. Portanto, pelo Teorema 5.10, temos que $1+c_{i} \partial^{e_{i}}+c_{j} \partial^{e_{j}}$ preserva estabilidade real.

Corolário 5.14. Seja $p \in \mathbb{R}\left[x_{1}, \ldots, x_{m}\right]$ um polinômio real estável e $i \in[m]$. Então $\left(1-\partial_{x_{i}}\right) p(x)$ é real estável.

Demonstração. Sejam $x_{1}, \ldots, x_{m}, z_{1}, \ldots, z_{m}$ indeterminadas algebricamente independentes. Sejam $A, B \subseteq \mathbb{N}^{m} \operatorname{com} A:=\{0\}$ e $B:=\left\{0, e_{i}\right\}$. Seja $c: B \rightarrow \mathbb{R}$ tal que $c(0)=1$ e $c\left(e_{i}\right)=-1$. Note que

$$
\sum_{\substack{\alpha \in A \\ \beta \in B}} c_{\beta} x^{\alpha} z^{\beta}=1-z_{i}
$$

Como $1-z_{i}$ é um polinômio real estável, então, pelo Teorema 5.10 , temos que $1-\partial^{e_{i}}$ preserva estabilidade real.

\subsection{Exemplos de polinômios estáveis}

Nesta seção, mostraremos exemplos de polinômios estáveis que serão utilizados para provar que algumas famílias de polinômios formam árvores entrelaçadoras de polinômios. Primeiramente, mostraremos que, para todas as matrizes positivas semidefinidas $A_{1}, \ldots, A_{m}$ e toda matriz hermitiana $B$, o polinômio $\operatorname{det}\left(\sum_{i \in[m]} x_{i} A_{i}+B\right) \in \mathbb{C}\left[x_{1}, \ldots, x_{m}\right]$ é real estável. Aplicaremos algumas operações que preservam a estabilidade real em polinômios dessa forma e, utilizando a caracterização de entrelaçamento comum que envolve estabilidade real, mostraremos que certas famílias de polinômios são entrelaçadoras.

Teorema 5.15. Sejam $A_{1}, A_{2}, \ldots, A_{m} \in \mathbb{C}^{d \times d}$ matrizes positivas semidefinidas e $B \in \mathbb{C}^{d \times d}$ uma matriz hermitiana. Se existe $i \in[m]$ tal que $A_{i} \succ 0$, então o polinômio

$$
\operatorname{det}\left(\sum_{i \in[m]} x_{i} A_{i}+B\right) \in \mathbb{C}\left[x_{1}, x_{2}, \ldots, x_{m}\right]
$$

é real estável.

Demonstração. Suponha que existe $i \in[m]$ tal que $A_{i} \succ 0$. Sejam $z \in \mathbb{R}_{++}^{m}$ e $y \in \mathbb{R}^{m}$. Pelo Teorema 5.5, temos que é suficiente provar que o polinômio definido por

$$
t \mapsto \operatorname{det}\left(\sum_{j \in[m]}\left(t z_{j}+y_{j}\right) A_{j}+B\right)
$$

possui apenas raízes reais. Defina $M:=\sum_{j \in[m]} z_{j} A_{j}$ e $C:=\sum_{j \in[m]} y_{j} A_{j}+B$. Note que $M \succ 0$ e também que

$$
\operatorname{det}\left(\sum_{j \in[m]}\left(t z_{j}+y_{j}\right) A_{j}+B\right)=\operatorname{det}\left(t \sum_{j \in[m]} z_{j} A_{j}+C\right)
$$




$$
\begin{aligned}
& =\operatorname{det}(t M+C) \\
& =\operatorname{det}\left(M^{1 / 2}\left(t I+M^{-1 / 2} C M^{-1 / 2}\right) M^{1 / 2}\right) \\
& =\operatorname{det}\left(M^{1 / 2}\right) \operatorname{det}\left(t I+M^{-1 / 2} C M^{-1 / 2}\right) \operatorname{det}\left(M^{1 / 2}\right) .
\end{aligned}
$$

Como $M \succ 0$, então $M^{1 / 2} \succ 0$, o que implica que $\operatorname{det}\left(M^{1 / 2}\right)>0$. Note que as matrizes $C$ e $M^{-1 / 2}$ são hermitianas e, portanto, $M^{-1 / 2} C M^{-1 / 2}$ é hermitiana. Assim, vale que $\operatorname{det}\left(t I+M^{-1 / 2} C M^{-1 / 2}\right)$ é o polinômio característico de uma matriz hermitiana, que possui somente raízes em $\mathbb{R}$. Portanto, o polinômio definido por

$$
t \mapsto \operatorname{det}\left(\sum_{j \in[m]}\left(t z_{j}+y_{j}\right) A_{j}+B\right)
$$

possui apenas raízes reais.

Corolário 5.16. Sejam $A_{1}, A_{2}, \ldots, A_{m} \in \mathbb{C}^{d \times d}$ matrizes positivas semidefinidas e $B \in \mathbb{C}^{d \times d}$ uma matriz hermitiana. Então o polinômio

$$
\operatorname{det}\left(\sum_{i \in[m]} x_{i} A_{i}+B\right) \in \mathbb{C}\left[x_{1}, x_{2}, \ldots, x_{m}\right]
$$

é real estável.

Demonstração. Se existe $i \in[m]$ tal que $A_{i} \succ 0$, é fácil ver que o resultado vale pelo Teorema 5.15. Suponha que $A_{i}$ não é positiva definida para todo $i \in[\mathrm{m}]$.

Seja $j \in[m]$. Pelo Teorema 2.39, temos que $A_{j}=\sum_{\lambda \in \operatorname{Spec}\left(A_{j}\right)} \lambda P_{E_{\lambda}}$. Defina

$$
C_{n}:=\frac{1}{n} P_{E_{0}}+\sum_{\lambda \in \operatorname{Spec}\left(A_{j}\right)} \lambda P_{E_{\lambda}}
$$

para todo $n \in \mathbb{N} \backslash\{0\}$. Note que $C_{n}$ é positiva definida para todo $n \in \mathbb{N} \backslash\{0\}$. Assim, pelo Teorema 5.15 o polinômio

$$
p_{n}:=\operatorname{det}\left(\sum_{i \in[m] \backslash\{j\}} x_{i} A_{i}+x_{j} C_{n}+B\right)
$$

é real estável para todo $n \in \mathbb{N} \backslash\{0\}$. Logo, vale que $p:=\operatorname{det}\left(\sum_{i \in[m]} x_{i} A_{i}+B\right)$ é um polinômio de coeficientes reais.

Suponha, por contradição, que o polinômio $p$ não é estável. Dessa forma, existe $y \in\left\{a \in \mathbb{C}^{m}\right.$ : $\left.\operatorname{Im}\left(a_{k}\right)>0 \forall k \in[m]\right\}$ tal que $p(y)=0$. Defina a função $f:\left\{x_{1}, x_{2}, \ldots, x_{m-1}\right\} \rightarrow\left\{y_{1}, y_{2}, \ldots, y_{m-1}\right\}$ como $f\left(x_{i}\right)=y_{i}$ para todo $i \in[m-1]$. Note que $y_{m}$ é raiz do polinômio monovariado $E_{f}(p)$. Além disso, como $p_{n}$ é real estável, então, pelo Teorema 5.6, vale que o polinômio monovariado $E_{f}\left(p_{n}\right)$ é estável para todo $n \in \mathbb{N} \backslash\{0\}$.

Pelo Teorema 4.16, temos que a sequência de polinômios $\left(E_{f}\left(p_{n}\right)\right)_{n \in \mathbb{N} \backslash\{0\}}$ converge uniformemente para $E_{f}(p)$ em todo subconjunto compacto de $\mathbb{C}$. Logo, pelo Corolário 4.15 , temos que $E_{f}(p)$ não tem raízes em $\{a \in \mathbb{C}: \operatorname{Im}(a)>0\}$. Portanto, $y_{m}$ não é raiz de $E_{f}(p)$ e $y$ não é raiz de $p$, o que nos leva a uma contradição. 
Para ilustrar esse resultado, considere as seguintes matrizes positivas semidefinidas:

$$
A_{1}=\left[\begin{array}{ll}
1 & 0 \\
0 & 1
\end{array}\right], \quad A_{2}=\left[\begin{array}{ll}
0 & 0 \\
0 & 1
\end{array}\right], \quad A_{3}=\left[\begin{array}{ll}
1 & 0 \\
0 & 0
\end{array}\right] .
$$

Pelo resultado apresentado acima, o polinômio em $\mathbb{R}\left[x_{1}, x_{2}, x_{3}\right]$ definido como

$$
\bar{q}:=\operatorname{det}\left(x_{1} A_{1}+x_{2} A_{2}+x_{3} A_{3}\right)
$$

é real estável. Note que $\bar{q}$ é o polinômio real estável $q\left(x_{1}, x_{2}, x_{3}\right)=x_{1}^{2} x_{2}+x_{1}^{2} x_{2} x_{3}$ definido anteriormente. Além disso, se um polinômio de grau $d$ é real estável e possui apenas duas indeterminadas $x_{1}$ e $x_{2}$, então ele pode ser escrito da seguinte forma: $\pm \operatorname{det}\left(x_{1} A+x_{2} B+C\right)$, onde $A, B \in \mathbb{C}^{d \times d}$ são matrizes positivas semidefinidas e $C \in \mathbb{C}^{d \times d}$ é uma matriz hermitiana. Assim, juntando esse resultado ao Corolário 5.16, obtemos uma caracterização de polinômios reais estáveis com duas indeterminadas: um polinômio de grau $d$ em $\mathbb{R}\left[x_{1}, x_{2}\right]$ é real estável se e somente se existem matrizes positivas semidefinidas $A, B \in \mathbb{C}^{d \times d}$ e uma matriz hermitiana $C \in \mathbb{C}^{d \times d}$ tais que $p\left(x_{1}, x_{2}\right)= \pm \operatorname{det}\left(x_{1} A+x_{2} B+C\right)$.

Teorema 5.17 (vide [22]). Seja $p \in \mathbb{R}\left[x_{1}, x_{2}\right]$ um polinômio real estável de grau $d$. Então existem $A, B, C \in \mathbb{C}^{d \times d}$, com $A, B$ positivas semidefinidas e $C$ hermitiana tais que

$$
p\left(x_{1}, x_{2}\right)= \pm \operatorname{det}\left(x_{1} A+x_{2} B+C\right) .
$$

Utilizaremos o resultado de Lewis e Parrilo [22] apresentado acima como caixa-preta.

A seguir, mostraremos que a soma ponderada das matrizes positivas semidefinidas referentes ao resultado acima é positiva definida. Esse resultado segue do Teorema 5.17.

Corolário 5.18. Seja $p \in \mathbb{R}\left[x_{1}, x_{2}\right]$ um polinômio real estável de grau $d$. Então existem $A, B, C \in$ $\mathbb{C}^{d \times d}$, com $A, B$ positivas semidefinidas e $C$ hermitiana tais que

$$
p\left(x_{1}, x_{2}\right)= \pm \operatorname{det}\left(x_{1} A+x_{2} B+C\right)
$$

e $\alpha A+\beta B \succ 0$ para todos $\alpha, \beta \in \mathbb{R}_{++}$.

Demonstração. Pelo Teorema 5.17, existem $A, B, C \in \mathbb{C}^{d \times d}$, com $A, B \succeq 0$ e $C$ hermitiana tais que $p\left(x_{1}, x_{2}\right)= \pm \operatorname{det}\left(x_{1} A+x_{2} B+C\right)$.

Suponha, por contradição, que existem $\alpha, \beta \in \mathbb{R}_{++}$tais que $\alpha A+\beta B$ não é positiva definida. Como $A, B \succeq 0$, então vale que $\alpha A+\beta B \succeq 0$. Dessa forma, existe $v \in \mathbb{R}^{d} \backslash\{0\}$ tal que

$$
v^{*}(\alpha A+\beta B) v=0 .
$$

Assim, vale que $\alpha v^{*} A v+\beta v^{*} B v=0$. Como $\alpha, \beta \in \mathbb{R}_{++}$e, além disso, $\alpha v^{*} A v \geq 0$ e $\beta v^{*} B v \geq 0$, então

$$
v^{*} A v=v^{*} B v=0 .
$$

Dessa forma, existem $i, j \in[d]$ tais que $\lambda_{i}^{\downarrow}(A)=\lambda_{j}^{\downarrow}(B)=0$.

Sejam $u_{1}, \ldots, u_{d}$ autovetores ortonormais de $A$ associados a $\lambda_{1}^{\downarrow}(A), \ldots, \lambda_{d}^{\downarrow}(A)$, respectivamente. Seja $Q \in \mathbb{C}^{d \times d}$ tal que $Q^{*} e_{i}=u_{i}$ para todo $i \in[d]$. Note que $Q Q^{*}=I$. Pelo Teorema 2.39, temos que

$$
A=\sum_{i \in[d]} \lambda_{i}^{\downarrow}(A) u_{i} u_{i}^{*}
$$


Assim, vale que $Q A Q^{*}=\operatorname{Diag}\left(\lambda^{\downarrow}(A)\right)=: D$. Note que

$$
\begin{aligned}
\operatorname{det}\left(x_{1} A+x_{2} B+C\right) & =\operatorname{det}\left(Q^{*}\right) \operatorname{det}\left(Q\left(x_{1} A+x_{2} B+C\right) Q^{*}\right) \operatorname{det}(Q) \\
& =\operatorname{det}\left(Q^{*}\right) \operatorname{det}\left(x_{1} D+x_{2} Q B Q^{*}+Q C Q^{*}\right) \operatorname{det}(Q) \\
& =\operatorname{det}\left(x_{1} D+x_{2} Q B Q^{*}+Q C Q^{*}\right) .
\end{aligned}
$$

Como existe $i \in[d]$ tal que $\lambda_{i}^{\downarrow}(A)=0$, então $D_{i, i}=0$. Assim, vale que

$$
\left[x_{1}^{d}\right]\left(\operatorname{det}\left(x_{1} D+x_{2} Q B Q^{*}+Q C Q^{*}\right)\right)=0,
$$

o que implica que $\left[x_{1}^{d}\right](p)=0$. Analogamente, temos que $\left[x_{2}^{d}\right](p)=0$ e, portanto, o polinômio $p$ não tem grau $d$, o que nos leva a uma contradição.

No Capítulo 4, mostramos que existe uma caracterização de árvores entrelaçadoras que envolve a estabilidade real de certas combinações convexas dos polinômios dessa árvore. Utilizaremos essa caracterização para mostrar que certas famílias de polinômios são entrelaçadoras fazendo uso de alguns exemplos de polinômios reais estáveis de formatos mais específicos. O primeiro exemplo de polinômio real estável é o polinômio $p \in \mathbb{C}[x]$, definido como

$$
p(x):=\sum_{S \subseteq[m]}\left(\prod_{j \in S} \lambda_{j}\right)\left(\prod_{j \in[m] \backslash S}\left(1-\lambda_{j}\right)\right) \operatorname{det}\left(x I+A+\sum_{j \in S} u_{j} u_{j}^{\top}+\sum_{j \in[m] \backslash S} v_{j} v_{j}^{\top}\right),
$$

onde $A \in \mathbb{C}^{n \times n}$ é positiva semidefinida, $m \in \mathbb{Z}_{++}, u_{1}, \ldots, u_{m}, v_{1}, \ldots, v_{m} \in \mathbb{R}^{n}$ e $\lambda_{1}, \ldots, \lambda_{m} \in[0,1]$. Esse polinômio será utilizado para mostrar que a família formada por polinômios característicos das matrizes de sinais de um grafo forma uma árvore entrelaçadora de polinômios, onde matrizes de sinais são basicamente matrizes de adjacência cujos elementos possuem sinais positivos ou negativos. Apresentaremos, por indução, uma forma diferente de representar o polinômio $p$, de modo que seja mais simples mostrar que ele é real estável. Para isso, mostraremos um resultado intermediário, que será apresentado a seguir.

Lema 5.19 (vide [24, Teorema 6.5]). Sejam $A \in \mathbb{C}^{n \times n}$ uma matriz inversível e $x_{1}, x_{2}$ indeterminadas algebricamente independentes. Sejam $u, v \in \mathbb{C}^{n}$ e um número real $\lambda \in[0,1]$. Então

$E_{x_{1}=x_{2}=0}\left(\left(1+\lambda \partial_{x_{1}}+(1-\lambda) \partial_{x_{2}}\right)\left(\operatorname{det}\left(A+x_{1} u u^{\top}+x_{2} v v^{\top}\right)\right)\right)=\lambda \operatorname{det}\left(A+u u^{\top}\right)+(1-\lambda) \operatorname{det}\left(A+v v^{\top}\right)$.

Demonstração. Pelo Lema 2.18, temos que

$$
\begin{aligned}
& \operatorname{det}\left(A+x_{1} u u^{\top}\right)=\operatorname{det}(A)\left(1+x_{1} u^{\top} A^{-1} u\right), \\
& \operatorname{det}\left(A+x_{2} v v^{\top}\right)=\operatorname{det}(A)\left(1+x_{2} v^{\top} A^{-1} v\right),
\end{aligned}
$$

o que implica que

$$
\begin{aligned}
& \partial_{x_{1}} \operatorname{det}\left(A+x_{1} u u^{\top}\right)=\operatorname{det}(A)\left(u^{\top} A^{-1} u\right), \\
& \partial_{x_{2}} \operatorname{det}\left(A+x_{2} v v^{\top}\right)=\operatorname{det}(A)\left(v^{\top} A^{-1} v\right) .
\end{aligned}
$$

Pela Proposição 2.11, temos que $E_{x_{1}=x_{2}=0}\left(\operatorname{det}\left(A+x_{1} u u^{\top}+x_{2} v v^{\top}\right)\right)=\operatorname{det}(A)$. Note também que, pelas Proposições 2.11 e 2.12, temos que

$$
E_{x_{1}=x_{2}=0}\left(\partial_{x_{1}} \operatorname{det}\left(A+x_{1} u u^{\top}+x_{2} v v^{\top}\right)\right)=E_{x_{1}=0} \circ \partial_{x_{1}} \circ E_{x_{2}=0} \operatorname{det}\left(A+x_{1} u u^{\top}+x_{2} v v^{\top}\right)
$$




$$
\begin{aligned}
& =E_{x_{1}=0} \circ \partial_{x_{1}} \operatorname{det}\left(A+x_{1} u u^{\top}\right) \\
& =\operatorname{det}(A)\left(u^{\top} A^{-1} u\right) \\
E_{x_{1}=x_{2}=0}\left(\partial_{x_{2}} \operatorname{det}\left(A+x_{1} u u^{\top}+x_{2} v v^{\top}\right)\right) & =E_{x_{2}=0} \circ \partial_{x_{2}} \circ E_{x_{1}=0} \operatorname{det}\left(A+x_{1} u u^{\top}+x_{2} v v^{\top}\right) \\
& =E_{x_{2}=0} \circ \partial_{x_{2}} \operatorname{det}\left(A+x_{2} v v^{\top}\right) \\
& =\operatorname{det}(A)\left(v^{\top} A^{-1} v\right)
\end{aligned}
$$

Portanto, vale que

$$
\begin{aligned}
E_{x_{1}=x_{2}=0}\left(\left(1+\lambda \partial_{x_{1}}\right.\right. & \left.\left.+(1-\lambda) \partial_{x_{2}}\right)\left(\operatorname{det}\left(A+x_{1} u u^{\top}+x_{2} v v^{\top}\right)\right)\right) \\
& =\operatorname{det}(A)+\lambda \operatorname{det}(A)\left(u^{\top} A^{-1} u\right)+(1-\lambda) \operatorname{det}(A)\left(v^{\top} A^{-1} v\right) \\
& =\lambda \operatorname{det}(A)\left(1+u^{\top} A^{-1} u\right)+(1-\lambda) \operatorname{det}(A)\left(1+v^{\top} A^{-1} v\right) \\
& =\lambda \operatorname{det}\left(A+u u^{\top}\right)+(1-\lambda) \operatorname{det}\left(A+v v^{\top}\right),
\end{aligned}
$$

onde a última igualdade vale pelo Lema 2.18.

Apresentaremos a seguir outra forma de representar o polinômio $p$ definido anteriormente.

Lema 5.20 (vide [24]). Sejam $u_{1}, \ldots, u_{m}, v_{1}, \ldots, v_{m} \in \mathbb{R}^{n}$ e números reais $\lambda_{1}, \ldots, \lambda_{m} \in[0,1]$. Seja $k \in\{0, \ldots, m\}$ e seja $A \in \mathbb{C}^{n \times n} \operatorname{com} A \succeq 0$. Defina $p_{k} \in \mathbb{C}\left[x, y_{1}, \ldots, y_{m}, z_{1}, \ldots, z_{m}\right]$ como

$$
p_{k}:=\sum_{S \subseteq[k]}\left(\prod_{j \in S} \lambda_{j}\right)\left(\prod_{j \in[k] \backslash S}\left(1-\lambda_{j}\right)\right) \operatorname{det}\left(x I+A+\sum_{j \in S} u_{j} u_{j}^{\top}+\sum_{j \in[k] \backslash S} v_{j} v_{j}^{\top}+\sum_{j=k+1}^{m}\left(y_{j} u_{j} u_{j}^{\top}+z_{j} v_{j} v_{j}^{\top}\right)\right),
$$

e $T_{\ell}:=1+\lambda_{\ell} \partial_{y_{\ell}}+\left(1-\lambda_{k}\right) \partial_{z_{\ell}}$ para todo $\ell \in[m]$. Então

$$
p_{k}=\left(\prod_{j \in[k]} E_{y_{j}=z_{j}=0} \circ T_{j}\right) \operatorname{det}\left(x I+A+\sum_{j \in[m]} y_{j} u_{j} u_{j}^{\top}+\sum_{j \in[m]} z_{j} v_{j} v_{j}^{\top}\right) .
$$

Demonstração. Provaremos por indução em $k$. Se $k=0$, é fácil ver que

$$
p_{0}=\operatorname{det}\left(x I+A+\sum_{j \in[m]} y_{j} u_{j} u_{j}^{\top}+\sum_{j \in[m]} z_{j} v_{j} v_{j}^{\top}\right)
$$

Suponha agora que $k \geq 1$. Para todo $\ell \in\{k-1, k\}$, defina $q_{\ell} \in \mathbb{C}\left[x, y_{1}, \ldots, y_{n}, z_{1}, \ldots, y_{n}\right]$ como

$$
q_{\ell}:=\left(\prod_{j \in[\ell]} E_{y_{j}=z_{j}=0} \circ T_{j}\right) \operatorname{det}\left(x I+A+\sum_{j \in[m]} y_{j} u_{j} u_{j}^{\top}+\sum_{j \in[m]} z_{j} v_{j} v_{j}^{\top}\right) .
$$

Pela hipótese de indução, temos que $p_{k-1}=q_{k-1}$. Logo, temos que

$$
q_{k}=\left(E_{y_{k}=z_{k}=0} \circ T_{k}\right) p_{k-1} .
$$

Sejam $\alpha, \beta:[m] \rightarrow \mathbb{C}$. Defina $C_{S} \in \mathbb{C}^{n \times n}[x]$ como

$$
C_{S}(x):=x I+A+\sum_{j \in S} u_{j} u_{j}^{\top}+\sum_{j \in[k-1] \backslash S} v_{j} v_{j}^{\top}+\sum_{j=k+1}^{m}\left(\alpha_{j} u_{j} u_{j}^{\top}+\beta_{j} v_{j} v_{j}^{\top}\right)
$$


para todo $S \subseteq[m]$. Vale que existe $\Gamma \subseteq \mathbb{R}$ de tamanho infinito tal que $E_{x=\gamma}\left(C_{S}\right)$ é inversível para todo $\gamma \in \Gamma$. Seja $S \subseteq[m]$. Pelo Lema 5.19, temos que, para todo $\gamma \in \Gamma$, vale que

$$
\begin{aligned}
\left(E_{y_{k}=z_{k}=0} \circ T_{k}\right) \operatorname{det}\left(C_{S}(\gamma)+y_{k} u_{k} u_{k}^{\top}+z_{k} v_{k} v_{k}^{\top}\right) \\
\quad=\lambda_{k} \operatorname{det}\left(C_{S}(\gamma)+u_{k} u_{k}^{\top}\right)+\left(1-\lambda_{k}\right) \operatorname{det}\left(C_{S}(\gamma)+v_{k} v_{k}^{\top}\right) .
\end{aligned}
$$

Logo, como os polinômios

$$
\begin{gathered}
\left(E_{y_{k}=z_{k}=0} \circ T_{k}\right) \operatorname{det}\left(C_{S}(x)+y_{k} u_{k} u_{k}^{\top}+z_{k} v_{k} v_{k}^{\top}\right), \\
\lambda_{k} \operatorname{det}\left(C_{S}(x)+u_{k} u_{k}^{\top}\right)+\left(1-\lambda_{k}\right) \operatorname{det}\left(C_{S}(x)+v_{k} v_{k}^{\top}\right)
\end{gathered}
$$

pertencem a $\mathbb{C}[x]$ e $\Gamma$ tem tamanho infinito, então

$$
\left(E_{y_{k}=z_{k}=0} \circ T_{k}\right) \operatorname{det}\left(C_{S}(x)+y_{k} u_{k} u_{k}^{\top}+z_{k} v_{k} v_{k}^{\top}\right)=\lambda_{k} \operatorname{det}\left(C_{S}(x)+u_{k} u_{k}^{\top}\right)+\left(1-\lambda_{k}\right) \operatorname{det}\left(C_{S}(x)+v_{k} v_{k}^{\top}\right),
$$

o que implica que

$$
\begin{aligned}
\left(E_{y_{k}=z_{k}=0} \circ T_{k}\right) \operatorname{det}\left(x I+A+\sum_{j \in S} u_{j} u_{j}^{\top}+\sum_{j \in[k-1] \backslash S} v_{j} v_{j}^{\top}+\sum_{j=k}^{m}\left(y_{j} u_{j} u_{j}^{\top}+z_{j} v_{j} v_{j}^{\top}\right)\right) \\
=\lambda_{k} \operatorname{det}\left(x I+A+\sum_{j \in S} u_{j} u_{j}^{\top}+\sum_{j \in[k-1] \backslash S} v_{j} v_{j}^{\top}+\sum_{j=k+1}^{m}\left(y_{j} u_{j} u_{j}^{\top}+z_{j} v_{j} v_{j}^{\top}\right)+u_{k} u_{k}^{\top}\right) \\
\quad+\left(1-\lambda_{k}\right) \operatorname{det}\left(x I+A+\sum_{j \in S} u_{j} u_{j}^{\top}+\sum_{j \in[k-1] \backslash S} v_{j} v_{j}^{\top}+\sum_{j=k+1}^{m}\left(y_{j} u_{j} u_{j}^{\top}+z_{j} v_{j} v_{j}^{\top}\right)+v_{k} v_{k}^{\top}\right) \\
=\lambda_{k} \operatorname{det}\left(x I+A+\sum_{j \in S \cup\{k\}} u_{j} u_{j}^{\top}+\sum_{j \in[k-1] \backslash S} v_{j} v_{j}^{\top}+\sum_{j=k+1}^{m}\left(y_{j} u_{j} u_{j}^{\top}+z_{j} v_{j} v_{j}^{\top}\right)\right) \\
\quad+\left(1-\lambda_{k}\right) \operatorname{det}\left(x I+A+\sum_{j \in S} u_{j} u_{j}^{\top}+\sum_{j \in([k-1] \backslash S) \cup\{k\}} v_{j} v_{j}^{\top}+\sum_{j=k+1}^{m}\left(y_{j} u_{j} u_{j}^{\top}+z_{j} v_{j} v_{j}^{\top}\right)\right) .
\end{aligned}
$$

Assim, vale que

$$
\begin{aligned}
q_{k}= & \left(E_{y_{k}=z_{k}=0} \circ T_{k}\right) p_{k-1} \\
= & \sum_{S \subseteq[k-1]}\left(\prod_{j \in S} \lambda_{j}\right)\left(\prod_{j \in[k-1] \backslash S}\left(1-\lambda_{j}\right)\right)\left(\lambda _ { k } \operatorname { d e t } \left(x I+A+\sum_{j \in S \cup\{k\}} u_{j} u_{j}^{\top}\right.\right. \\
& \left.+\sum_{j \in[k-1] \backslash S} v_{j} v_{j}^{\top}+\sum_{j=k+1}^{m}\left(y_{j} u_{j} u_{j}^{\top}+z_{j} v_{j} v_{j}^{\top}\right)\right) \\
& \left.+\left(1-\lambda_{k}\right) \operatorname{det}\left(x I+A+\sum_{j \in S} u_{j} u_{j}^{\top}+\sum_{j \in[k] \backslash S} v_{j} v_{j}^{\top}+\sum_{j=k+1}^{m}\left(y_{j} u_{j} u_{j}^{\top}+z_{j} v_{j} v_{j}^{\top}\right)\right)\right) \\
= & \sum_{S \subseteq[k-1]}\left(\prod_{j \in S \cup\{k\}} \lambda_{j}\right)\left(\prod_{j \in[k-1] \backslash S}\left(1-\lambda_{j}\right)\right)\left(\operatorname { d e t } \left(x I+A+\sum_{j \in S \cup\{k\}} u_{j} u_{j}^{\top}\right.\right.
\end{aligned}
$$




$$
\begin{aligned}
& \left.\left.+\sum_{j \in[k-1] \backslash S} v_{j} v_{j}^{\top}+\sum_{j=k+1}^{m}\left(y_{j} u_{j} u_{j}^{\top}+z_{j} v_{j} v_{j}^{\top}\right)\right)\right) \\
& +\sum_{S \subseteq[k-1]}\left(\prod_{j \in S} \lambda_{j}\right)\left(\prod_{j \in[k] \backslash S}\left(1-\lambda_{j}\right)\right)\left(\operatorname { d e t } \left(x I+A+\sum_{j \in S} u_{j} u_{j}^{\top}\right.\right. \\
& \left.\left.+\sum_{j \in[k] \backslash S} v_{j} v_{j}^{\top}+\sum_{j=k+1}^{m}\left(y_{j} u_{j} u_{j}^{\top}+z_{j} v_{j} v_{j}^{\top}\right)\right)\right) \\
& =\sum_{S \subseteq[k]}\left(\prod_{j \in S} \lambda_{j}\right)\left(\prod_{j \in[k] \backslash S}\left(1-\lambda_{j}\right)\right)\left(\operatorname { d e t } \left(x I+A+\sum_{j \in S} u_{j} u_{j}^{\top}\right.\right. \\
& \left.+\sum_{j \in[k] \backslash S} v_{j} v_{j}^{\top}+\sum_{j=k+1}^{m}\left(y_{j} u_{j} u_{j}^{\top}+z_{j} v_{j} v_{j}^{\top}\right)\right) \\
& =p_{k} .
\end{aligned}
$$

Pelo resultado acima, podemos considerar que o polinômio $p$ pode ser representado da seguinte maneira:

$$
p(x)=\left(\prod_{j \in[m]} E_{y_{j}=z_{j}=0} \circ T_{j}\right) \operatorname{det}\left(x I+A+\sum_{j \in[m]} y_{j} u_{j} u_{j}^{\top}+\sum_{j \in[m]} z_{j} v_{j} v_{j}^{\top}\right),
$$

onde $T_{k}:=1+\lambda_{k} \partial_{y_{k}}+\left(1-\lambda_{k}\right) \partial_{z_{k}}$ e $y_{k}, z_{k}$ são indeterminadas para todo $k \in[m]$. Note que $p$ resulta de $m$ operações aplicadas sobre um polinômio $q \in \mathbb{C}\left[x, y_{1}, \ldots, y_{m}, z_{1}, \ldots, z_{m}\right]$ formado pelo determinante de uma matriz com indeterminadas. Podemos utilizar o Corolário 5.16 para mostrar que $q$ é um polinômio real estável. Além disso, utilizamos composições de algumas operações que preservam a estabilidade real, vistas anteriormente, para mostrar que as $m$ operações aplicadas sobre $q$ também preservam a estabilidade real. Inclusive, uma combinação de operações que preservam estabilidade real transforma o polinômio $q$ em $p$. Dessa forma, vale que $p$ é real estável, como será mostrado com mais detalhes a seguir.

Lema 5.21 (Marcus, Spielman e Srivastava [24, Teorema 6.6]). Sejam $u_{1}, \ldots, u_{m}, v_{1}, \ldots, v_{m} \in \mathbb{R}^{n}$ e números reais $\lambda_{1}, \ldots, \lambda_{m} \in[0,1]$. Seja $A \in \mathbb{C}^{n \times n} \operatorname{com} A \succeq 0$. Então o polinômio $p \in \mathbb{C}[x]$, definido por

$$
p(x):=\sum_{S \subseteq[m]}\left(\prod_{j \in S} \lambda_{j}\right)\left(\prod_{j \in[m] \backslash S}\left(1-\lambda_{j}\right)\right) \operatorname{det}\left(x I+A+\sum_{j \in S} u_{j} u_{j}^{\top}+\sum_{j \in[m] \backslash S} v_{j} v_{j}^{\top}\right),
$$

é real estável.

Demonstração. Sejam $y_{1}, \ldots, y_{m}, z_{1}, \ldots, z_{m}$ indeterminadas algebricamente independentes. Defina $T_{k}:=1+\lambda_{k} \partial_{y_{k}}+\left(1-\lambda_{k}\right) \partial_{z_{k}}$ para todo $k \in[m]$. Pelo Lema 5.20, temos que

$$
p(x)=\left(\prod_{j \in[m]} E_{y_{j}=z_{j}=0} \circ T_{j}\right) \operatorname{det}\left(x I+A+\sum_{j \in[m]} y_{j} u_{j} u_{j}^{\top}+\sum_{j \in[m]} z_{j} v_{j} v_{j}^{\top}\right) .
$$

Pelo Corolário 5.16, temos que o polinômio $q \in \mathbb{C}\left[x, y_{1}, \ldots, y_{m}, z_{1}, \ldots, z_{m}\right]$ definido por

$$
q\left(x, y_{1}, \ldots, y_{m}, z_{1}, \ldots, z_{m}\right):=\operatorname{det}\left(x I+A+\sum_{j \in[m]} y_{j} u_{j} u_{j}^{\top}+\sum_{j \in[m]} z_{j} v_{j} v_{j}^{\top}\right)
$$


é real estável. Portanto, pelo Corolário 5.7(d) e pelo Corolário 5.13, temos que o polinômio

$$
\left(\prod_{j \in[m]} E_{y_{j}=z_{j}=0} \circ T_{j}\right) q\left(x, y_{1}, \ldots, y_{m}, z_{1}, \ldots, z_{m}\right)
$$

é real estável.

Utilizaremos o fato de $p$ ser real estável para mostrar que certos polinômios obtidos de matrizes de sinais, que serão definidas a seguir, também são reais estáveis. A estabilidade real desses polinômios será utilizada juntamente com o resultado de Chudnovsky e Seymour para mostrar que os polinômios característicos das matrizes de sinais de um grafo formam uma árvore entrelaçadora de polinômios.

\subsubsection{Matrizes de sinais}

Matrizes de sinais são matrizes reais associadas a grafos e a funções que atribuem um sinal positivo $(+1)$ ou negativo $(-1)$ para cada uma das arestas do grafo associado. Essas matrizes simétricas são indexadas pelos vértices do grafo associado e suas entradas pertencem ao conjunto $\{-1,0,1\}$, de forma que a entrada referente a dois vértices não vizinhos é zero e a entrada referente a dois vértices vizinhos é dada pela função de sinais. Dessa forma, os valores absolutos de cada uma das entradas da matriz de sinais de um grafo são iguais às respectivas entradas da matriz de adjacência desse grafo. No próximo capítulo, ilustraremos melhor o conceito de matrizes de sinais mostrando alguns exemplos.

Definição 5.22 (Matriz de sinais). Sejam $G=(V, E)$ um grafo e $s: E \rightarrow\{ \pm 1\}$. Defina a matriz de sinais $A_{G, s} \in\{-1,0,1\}^{V \times V}$ como

$$
A_{G, s}(u, v):= \begin{cases}s(\{u, v\}), & \text { se }\{u, v\} \in E, \\ 0, & \text { se }\{u, v\} \notin E\end{cases}
$$

para todos $u, v \in V$. Defina também $v_{S}:=2 \mathbb{1}_{S}-\mathbb{1}$ para todo $S \subseteq E$.

Ao decompor matrizes de sinais em somas de matrizes diagonais e matrizes de posto 1, fica fácil ver que o polinômio $q \in \mathbb{R}[x]$ definido como

$$
q(x):=\sum_{S \subseteq E}\left(\prod_{j \in S} \lambda_{j}\right)\left(\prod_{j \in E \backslash S}\left(1-\lambda_{j}\right)\right) \operatorname{det}\left(x I-A_{G, v_{S}}\right)
$$

possui o mesmo formato do polinômio $p$ mostrado anteriormente e, portanto, vale que $q$ é real estável.

Teorema 5.23 (Marcus, Spielman e Srivastava [24, Teorema 5.1]). Sejam $G=(V, E)$ um grafo e números reais $\lambda_{e} \in[0,1]$ para todo $e \in E$. Defina $v_{S}:=2 \mathbb{1}_{S}-\mathbb{1}$ para todo $S \subseteq E$. Então o polinômio $p \in \mathbb{R}[x]$, definido por

$$
p(x):=\sum_{S \subseteq E}\left(\prod_{j \in S} \lambda_{j}\right)\left(\prod_{j \in E \backslash S}\left(1-\lambda_{j}\right)\right) \operatorname{det}\left(x I-A_{G, v_{S}}\right),
$$

é real estável. 
Demonstração. Seja $d \in \mathbb{R}_{+}^{V}$ o vetor de graus de $G$. Defina $L_{G, v_{S}}:=\operatorname{Diag}(d)-A_{G, v_{S}}$ para todo $S \subseteq E$. Defina $D:=\Delta(G) I-\operatorname{Diag}(d) \succeq 0$. Seja $S \subseteq E$. Note que

$$
\Delta(G) I-A_{G, v_{S}}=D+L_{G, v_{S}} .
$$

Note também que

$$
L_{G, v_{S}}=\sum_{\{u, v\} \in S}\left(e_{v}-e_{u}\right)\left(e_{v}-e_{u}\right)^{\top}+\sum_{\{u, v\} \in E \backslash S}\left(e_{v}+e_{u}\right)\left(e_{v}+e_{u}\right)^{\top} .
$$

Logo, pela Proposição 2.11, temos que

$$
\begin{aligned}
E_{x=\Delta(G)+x}(p)= & \sum_{S \subseteq E}\left(\prod_{j \in S} \lambda_{j}\right)\left(\prod_{j \in E \backslash S}\left(1-\lambda_{j}\right)\right) \operatorname{det}\left(x I+\Delta(G) I-A_{G, v_{S}}\right) \\
= & \sum_{S \subseteq E}\left(\prod_{j \in S} \lambda_{j}\right)\left(\prod_{j \in E \backslash S}\left(1-\lambda_{j}\right)\right) \operatorname{det}\left(x I+D+L_{G, v_{S}}\right) \\
= & \sum_{S \subseteq E}\left(\prod_{j \in S} \lambda_{j}\right)\left(\prod_{j \in E \backslash S}\left(1-\lambda_{j}\right)\right) \operatorname{det}\left(x I+D+\sum_{\{u, v\} \in S}\left(e_{v}-e_{u}\right)\left(e_{v}-e_{u}\right)^{\top}\right. \\
& \left.+\sum_{\{u, v\} \in E \backslash S}\left(e_{v}+e_{u}\right)\left(e_{v}+e_{u}\right)^{\top}\right) .
\end{aligned}
$$

Assim, pelo Lema 5.21, temos que $E_{x=\Delta(G)+x}(p)$ é real estável. Portanto, pelo Corolário 5.7(b), temos que

$$
E_{x=x-\Delta(G)}\left(E_{x=\Delta(G)+x}(p)\right)
$$

é real estável, o que implica que $p$ é real estável.

Neste capítulo, utilizaremos a caracterização de entrelaçamento comum de Chudnovsky e Seymour juntamente com o resultado apresentado acima para mostrar que $\left\{\operatorname{det}\left(x I-A_{G, s}\right)\right\}_{s \in\{-1,1\}^{E}}$, que é a família de polinômios característicos de matrizes de sinais associadas a um grafo $G$, forma uma árvore entrelaçadora de polinômios. Assim, podemos utilizar a propriedade de árvores entrelaçadoras de polinômios para limitar superiormente as raízes de pelo menos um dos polinômios dessa família e, consequentemente, limitar superiormente os autovalores de certos grafos, mostrando a existência de famílias infinitas de grafos de Ramanujan.

Na seção a seguir, mostraremos outro exemplo de polinômios reais estáveis.

\subsubsection{Mixed Characteristic Polynomial}

Mixed Characteristic Polynomial é um polinômio monovariado associado a uma coleção de matrizes quadradas cujo formato se assemelha a polinômios característicos. Apesar da semelhança com polinômios característicos, o Mixed Characteristic Polynomial associado a uma única matriz não é equivalente ao polinômio característico da matriz em questão. Além disso, apesar de serem polinômios monovariados, eles são construídos a partir de especificações de polinômios multivariados, onde cada indeterminada é associada a uma matriz da coleção em questão. Segue a definição de Mixed Characteristic Polynomial.

Definição 5.24 (Mixed Characteristic Polynomial). Sejam $A_{1}, \ldots, A_{m} \in \mathbb{C}^{n \times n}$. Defina o Mixed Characteristic Polynomial $\mu\left[A_{1}, A_{2}, \ldots, A_{m}\right] \in \mathbb{C}[x]$ como

$$
\mu\left[A_{1}, A_{2}, \ldots, A_{m}\right](x):=E_{z_{1}=z_{2}=\cdots=z_{m}=0}\left(\left(\prod_{i \in[m]}\left(1-\partial_{z_{i}}\right)\right) \operatorname{det}\left(x I+\sum_{i \in[m]} z_{i} A_{i}\right)\right) .
$$


Esses polinômios, se associados a coleções de matrizes positivas semidefinidas, possuem algumas propriedades que serão utilizadas mais adiante na prova de uma solução do Problema de KadisonSinger.

A primeira propriedade é que Mixed Characteristic Polynomials de coleções de matrizes positivas semidefinidas são reais estáveis e, como estes são monovariados, então podemos dizer que possuem apenas raízes reais. Essa propriedade pode ser mostrada de forma simples utilizando um formato conhecido de polinômios estáveis, juntamente com operações que preservam a estabilidade real, ambos apresentados anteriormente neste capítulo.

Lema 5.25 (Marcus, Spielman e Srivastava [25]). Sejam $A_{1}, A_{2}, \ldots, A_{m} \in \mathbb{C}^{n \times n}$ matrizes positivas semidefinidas. Então $\mu\left[A_{1}, A_{2}, \ldots, A_{m}\right]$ possui somente raízes reais.

Demonstração. Sejam $x, z_{1}, \ldots, z_{m}$ indeterminadas algebricamente independentes. Pelo Corolário 5.16, temos que $\operatorname{det}\left(x I+\sum_{i \in[m]} z_{i} A_{i}\right)$ é real estável. Logo, pelo Corolário 5.14, temos que

$$
\left(\prod_{i \in[m]}\left(1-\partial_{z_{i}}\right)\right) \operatorname{det}\left(x I+\sum_{i \in[m]} z_{i} A_{i}\right)
$$

também é real estável. Portanto, pelo Corolário 5.7(d), temos que o polinômio monovariado

$$
E_{z_{1}=z_{2}=\cdots=z_{m}=0}\left(\left(\prod_{i \in[m]}\left(1-\partial_{z_{i}}\right)\right) \operatorname{det}\left(x I+\sum_{i \in[m]} z_{i} A_{i}\right)\right)
$$

é real estável, o que implica, pela Proposição 5.3, que ele possui somente raízes reais.

Outra propriedade é que podemos representar os Mixed Characteristic Polynomials de coleções de matrizes positivas semidefinidas de uma outra forma onde, ao contrário da forma original, os polinômios são construídos a partir de uma única indeterminada. Sejam $v_{1}, \ldots, v_{m}$ variáveis aleatórias independentes de suporte finito que assumem valores em $\mathbb{C}^{n}$ e sejam $A_{1}, \ldots, A_{m}$ matrizes positivas semidefinidas, definidas como $A_{i}:=\mathbb{E} v_{i} v_{i}^{*}$ para todo $i \in[m]$. Podemos representar o Mixed Characteristic Polynomial associado às matrizes $A_{1}, \ldots, A_{m}$ da seguinte forma:

$$
\mu\left[A_{1}, A_{2}, \ldots, A_{m}\right](x)=\mathbb{E}_{v_{1}, \ldots, v_{m}} \operatorname{det}\left(x I-\sum_{i \in[m]} v_{i} v_{i}^{*}\right) .
$$

Note que toda matriz positiva semidefinida pode ser representada como o valor esperado de uma variável aleatória que assume como valores matrizes semidefinidas de posto 1. Assim, o Mixed Characteristic Polynomial de qualquer coleção de matrizes positivas semidefinidas pode ser representado como o polinômio característico do valor esperado de $\sum_{i \in[m]} v_{i} v_{i}^{*}$. Essa representação encontra-se no Corolário 5.29 e, para obtê-la, utilizaremos uma série de resultados intermediários mostrados a seguir.

O resultado abaixo utiliza o conceito de suporte de variáveis aleatórias, que também será utilizado frequentemente ao longo do texto. Com o intuíto de simplificar a notação, faremos uso da definição a seguir. Sejam $(\Omega, P)$ um espaço de probabilidade discreto e $X: \Omega \rightarrow \mathbb{C}^{n}$ uma variável aleatória. Defina $R_{X}:=\{X(\omega): P(\omega)>0$ e $\omega \in \Omega\}$ como o suporte da variável aleatória $X$.

Lema 5.26. Sejam $A \in \mathbb{C}^{n \times n}$ uma matriz inversível e $v$ uma variável aleatória que assume valores em $\mathbb{C}^{n}$ com $R_{v}$ finito. Seja $x$ uma indeterminada. Então

$$
\mathbb{E}_{v} \operatorname{det}\left(A-v v^{*}\right)=E_{x=0}\left(\left(1-\partial_{x}\right) \operatorname{det}\left(A+x \mathbb{E}_{v} v v^{*}\right)\right) .
$$


Demonstração. Seja $p \in \mathbb{C}[x]$ o polinômio definido como $p(x)=\operatorname{det}\left(A+x \mathbb{E}_{v} v v^{*}\right)$. Note que

$$
\begin{aligned}
E_{x=0}\left(\left(1-\partial_{x}\right) p(x)\right) & =E_{x=0}(p(x))-E_{x=0}\left(\partial_{x} p(x)\right) \\
& =E_{x=0}\left(\operatorname{det}\left(A+x \mathbb{E}_{v} v v^{*}\right)\right)-E_{x=0}\left(\partial_{x} \operatorname{det}\left(A+x \mathbb{E}_{v} v v^{*}\right)\right) \\
& =\operatorname{det}(A)-E_{x=0}\left(\partial_{x} \operatorname{det}\left(A+x \mathbb{E}_{v} v v^{*}\right)\right) .
\end{aligned}
$$

Logo, como $A$ é inversível, pelo Lema 2.21, temos que

$$
\begin{aligned}
E_{x=0}\left(\left(1-\partial_{x}\right) p(x)\right) & =\operatorname{det}(A)-\operatorname{det}(A) \operatorname{Tr}\left(A^{-1} \mathbb{E}_{v} v v^{*}\right) \\
& =\operatorname{det}(A)\left(1-\operatorname{Tr}\left(A^{-1} \mathbb{E}_{v} v v^{*}\right)\right) .
\end{aligned}
$$

Portanto, pela Proposição 2.10, temos que

$$
\begin{aligned}
E_{x=0}\left(\left(1-\partial_{x}\right) p(x)\right) & =\operatorname{det}(A)\left(1-\mathbb{E}_{v} \operatorname{Tr}\left(A^{-1} v v^{*}\right)\right) \\
& =\mathbb{E}_{v} \operatorname{det}(A)\left(1-\operatorname{Tr}\left(A^{-1} v v^{*}\right)\right) \\
& =\mathbb{E}_{v} \operatorname{det}(A)\left(1-v^{*} A^{-1} v\right) \\
& =\mathbb{E}_{v} \operatorname{det}\left(A-v v^{*}\right),
\end{aligned}
$$

onde a última igualdade vale pelo Lema 2.18.

Segue o resultado do Lema 5.26 para matrizes hermitianas que não são necessariamente inversíveis.

Corolário 5.27. Sejam $A \in \mathbb{C}^{n \times n}$ uma matriz hermitiana e $v$ uma variável aleatória que assume valores em $\mathbb{C}^{n}$ com $R_{v}$ finito. Seja $x$ uma indeterminada. Então

$$
\mathbb{E}_{v} \operatorname{det}\left(A-v v^{*}\right)=E_{x=0}\left(\left(1-\partial_{x}\right) \operatorname{det}\left(A+x \mathbb{E}_{v} v v^{*}\right)\right) .
$$

Demonstração. Sejam $z \in \mathbb{R}_{++}$tal que $\lambda_{i}^{\downarrow}(A) \notin(0, z]$ para todo $i \in[n]$. Seja $N \in \mathbb{N} \backslash\{0\}$ tal que $\frac{1}{N}<z$. Defina $A_{n}:=A-\frac{1}{n} I$ para todo $n \in \mathbb{N}$ com $n \geq N$. Note que, para todo $n \in \mathbb{N}$ com $n \geq N$, vale que $A_{n}$ é inversível. Logo, pelo Lema 5.26, temos que

$$
\mathbb{E}_{v} \operatorname{det}\left(A_{n}-v v^{*}\right)=E_{x=0}\left(\left(1-\partial_{x}\right) \operatorname{det}\left(A_{n}+x \mathbb{E}_{v} v v^{*}\right)\right)
$$

para todo $n \in \mathbb{N}$ com $n \geq N$. Assim, como determinante é uma função contínua, então

$$
\begin{aligned}
\mathbb{E}_{v} \operatorname{det}\left(A-v v^{*}\right) & =\mathbb{E}_{v} \operatorname{det}\left(\lim _{n \rightarrow \infty} A_{n}-v v^{*}\right) \\
& =\lim _{n \rightarrow \infty} \mathbb{E}_{v} \operatorname{det}\left(A_{n}-v v^{*}\right) \\
& =\lim _{n \rightarrow \infty} E_{x=0}\left(\left(1-\partial_{x}\right) \operatorname{det}\left(A_{n}+x \mathbb{E}_{v} v v^{*}\right)\right) \\
& =E_{x=0}\left(\left(1-\partial_{x}\right) \operatorname{det}\left(\lim _{n \rightarrow \infty} A_{n}+x \mathbb{E}_{v} v v^{*}\right)\right) \\
& =E_{x=0}\left(\left(1-\partial_{x}\right) \operatorname{det}\left(A+x \mathbb{E}_{v} v v^{*}\right)\right) .
\end{aligned}
$$

Teorema 5.28 (Marcus, Spielman e Srivastava [25]). Sejam $v_{1}, v_{2}, \ldots, v_{m}$ variáveis aleatórias independentes que assumem valores em $\mathbb{C}^{n}$ com $R_{v_{i}}$ finito para todo $i \in[m]$. Defina $A_{i}:=\mathbb{E}_{v_{i}} v_{i} v_{i}^{*}$ para todo $i \in[m]$. Então vale a seguinte igualdade

$$
E_{x_{1}=\cdots=x_{m}=0}\left(\left(\prod_{i \in[m]}\left(1-\partial_{x_{i}}\right)\right) \operatorname{det}\left(A+\sum_{i \in[m]} x_{i} A_{i}\right)\right)=\mathbb{E}_{v_{1}, \ldots, v_{m}} \operatorname{det}\left(A-\sum_{i \in[m]} v_{i} v_{i}^{*}\right)
$$

para toda matriz hermitiana $A \in \mathbb{C}^{n \times n}$, onde $x_{1}, \ldots, x_{m}$ são indeterminadas algebricamente independentes. 
Demonstração. Provaremos esse resultado por indução em $m$. Se $m=0$, é fácil ver que o resultado vale. Suponha que $m \geq 1$. Seja $A \in \mathbb{C}^{n \times n}$ uma matriz hermitiana. Como $v_{1}, \ldots, v_{m}$ são variáveis aleatórias independentes, então

$$
\mathbb{E}_{v_{1}, \ldots, v_{m}} \operatorname{det}\left(A-\sum_{i \in[m]} v_{i} v_{i}^{*}\right)=\mathbb{E}_{v_{1}, \ldots, v_{m-1}} \mathbb{E}_{v_{m}} \operatorname{det}\left(A-\sum_{i \in[m-1]} v_{i} v_{i}^{*}-v_{m} v_{m}^{*}\right) .
$$

Logo, pela Proposição 2.10 e pelo Corolário 5.27, temos que

$$
\begin{aligned}
\mathbb{E}_{v_{1}, \ldots, v_{m}} \operatorname{det}\left(A-\sum_{i \in[m]} v_{i} v_{i}^{*}\right) & =\mathbb{E}_{v_{1}, \ldots, v_{m-1}} E_{x_{m}=0}\left(\left(1-\partial_{x_{m}}\right) \operatorname{det}\left(A-\sum_{i \in[m-1]} v_{i} v_{i}^{*}+x_{m} \mathbb{E}_{v_{m}} v_{m} v_{m}^{*}\right)\right) \\
& =E_{x_{m}=0}\left(\left(1-\partial_{x_{m}}\right) \mathbb{E}_{v_{1}, \ldots, v_{m-1}} \operatorname{det}\left(A+x_{m} A_{m}-\sum_{i \in[m-1]} v_{i} v_{i}^{*}\right)\right) .
\end{aligned}
$$

Seja $a \in \mathbb{C}$. Pela hipótese de indução, temos que

$$
\begin{aligned}
\mathbb{E}_{v_{1}, \ldots, v_{m-1}} \operatorname{det}\left(A+E_{x_{m}=a}\left(x_{m} A_{m}\right)-\sum_{i \in[m-1]} v_{i} v_{i}^{*}\right) \\
=E_{x_{1}=\cdots=x_{m-1}=0}\left(\left(\prod_{i \in[m-1]}\left(1-\partial_{x_{i}}\right)\right) \operatorname{det}\left(A+E_{x_{m}=a}\left(x_{m} A_{m}\right)+\sum_{i \in[m-1]} x_{i} A_{i}\right)\right) .
\end{aligned}
$$

Assim, pelas Proposições 2.8, 2.10, 2.11 e 2.12, temos que

$$
\begin{aligned}
E_{x_{m}=a} & \left(\mathbb{E}_{v_{1}, \ldots, v_{m-1}} \operatorname{det}\left(A+x_{m} A_{m}-\sum_{i \in[m-1]} v_{i} v_{i}^{*}\right)\right) \\
& =E_{x_{m}=a}\left(E_{x_{1}=\cdots=x_{m-1}=0}\left(\left(\prod_{i \in[m-1]}\left(1-\partial_{x_{i}}\right)\right) \operatorname{det}\left(A+x_{m} A_{m}+\sum_{i \in[m-1]} x_{i} A_{i}\right)\right)\right) .
\end{aligned}
$$

para todo $a \in \mathbb{C}$. Dessa forma, como os polinômios do lado esquerdo e do lado direito da igualdade acima são iguais para todos os valores complexos de $x_{m}$, então

$$
\begin{aligned}
\mathbb{E}_{v_{1}, \ldots, v_{m-1}} \operatorname{det}\left(A+x_{m} A_{m}-\right. & \left.\sum_{i \in[m-1]} v_{i} v_{i}^{*}\right) \\
=E_{x_{1}=\cdots=x_{m-1}=0} & \left(\left(\prod_{i \in[m-1]}\left(1-\partial_{x_{i}}\right)\right) \operatorname{det}\left(A+x_{m} A_{m}+\sum_{i \in[m-1]} x_{i} A_{i}\right)\right) .
\end{aligned}
$$

Logo, pela Proposição 2.12, vale que

$$
\begin{aligned}
& \mathbb{E}_{v_{1}, \ldots, v_{m}} \operatorname{det}\left(A-\sum_{i \in[m]} v_{i} v_{i}^{*}\right) \\
& =E_{x_{m}=0}\left(\left(1-\partial_{x_{m}}\right) E_{x_{1}=\cdots=x_{m-1}=0}\left(\prod_{i \in[m-1]}\left(1-\partial_{x_{i}}\right)\right) \operatorname{det}\left(A+x_{m} A_{m}+\sum_{i \in[m-1]} x_{i} A_{i}\right)\right) \\
& =E_{x_{1}=\cdots=x_{m}=0}\left(\left(\prod_{i \in[m]}\left(1-\partial_{x_{i}}\right)\right) \operatorname{det}\left(A+\sum_{i \in[m]} x_{i} A_{i}\right)\right) .
\end{aligned}
$$


Corolário 5.29. Sejam $v_{1}, v_{2}, \ldots, v_{m}$ variáveis aleatórias independentes que assumem valores em $\mathbb{C}^{n}$ com $R_{v_{i}}$ finito para todo $i \in[m]$. Defina $A_{i}:=\mathbb{E}_{v_{i}} v_{i} v_{i}^{*}$ para todo $i \in[m]$. Então

$$
\mu\left[A_{1}, A_{2}, \ldots, A_{m}\right](x)=\mathbb{E}_{v_{1}, \ldots, v_{m}} \operatorname{det}\left(x I-\sum_{i \in[m]} v_{i} v_{i}^{*}\right) .
$$

Demonstração. O resultado segue da Definição 5.24 e do Teorema 5.28.

\subsection{Aplicações em árvores entrelaçadoras de polinômios}

Como visto anteriormente, árvores entrelaçadoras de polinômios possuem uma importante propriedade: as raízes de pelo menos um dos seus polinômios folha são limitadas superiormente pela raiz máxima da soma de todos os seus polinômios folha. Mais adiante, utilizaremos essa propriedade para limitar superiormente os autovalores não triviais de uma família de grafos $d$-regulares por $2 \sqrt{d-1}$, mostrando que ela é uma família de grafos de Ramanujan. Além disso, também utilizaremos essa propriedade para limitar superiormente, por um valor desejado, os autovalores de pelo menos uma matriz de certos conjuntos de matrizes, provando uma das conjecturas de uma solução do Problema de Kadison-Singer. Para utilizar essa propriedade nas famílias de polinômios desejadas, mostraremos, a seguir, dois exemplos de árvores entrelaçadoras de polinômios utilizando a caracterização de entrelaçamento comum para determinar se uma árvore de polinômios é entrelaçadora. Assim, ao invés de mostrar que os polinômios desejados formam uma árvore entrelaçadora verificando todas as suas raízes, mostraremos que combinações convexas, com certos suportes, dos polinômios folha dessa árvore são reais estáveis. Anteriormente, mostramos exemplos de polinômios reais estáveis que, juntamente com a caracterização de entrelaçamento comum, nos permite concluir que os polinômios desejados formam árvores entrelaçadoras de polinômios, como mostrado nos Teoremas 5.30 e 5.32 .

Segue o primeiro exemplo de árvores entrelaçadoras.

Teorema 5.30 (Marcus, Spielman e Srivastava [24]). Seja $G=(V, E)$ um grafo. Então, a família de polinômios

$$
\left\{\operatorname{det}\left(x I-A_{G, s}\right)\right\}_{s \in\{ \pm 1\}^{E}}
$$

é uma família entrelaçadora de polinômios.

Demonstração. Defina

$$
\begin{gathered}
F_{s}:=\left\{t \in\{ \pm 1\}^{E}: t_{i}=s_{i} \forall i \in S\right\}, \\
p_{s}=\sum_{t \in F_{s}} \operatorname{det}\left(x I-A_{G, t}\right)
\end{gathered}
$$

para todo $S \subseteq E$ e todo $s \in\{ \pm 1\}^{S}$. Sejam $S \subseteq E$ e $s \in\{ \pm 1\}^{S}$. Sejam $a_{1}, \ldots, a_{m}$ as arestas de $G$.

Sejam $S_{0} \subsetneq S_{1} \subsetneq \cdots \subsetneq S_{m}=E$ tais que $\left|S_{i}\right|=i$ para todo $i \in[m]$. Para provar que $\left\{\operatorname{det}\left(x I-A_{G, s}\right)\right\}_{s \in\{ \pm 1\}^{E}}$ é uma família entrelaçadora de polinômios, mostraremos que, para todo $k \in[m]$ e todo $s \in\{ \pm 1\}^{S_{k-1}}$, os polinômios em

$$
\left\{p_{t}: t \in\{ \pm 1\}^{S_{k}} \text { e } t_{i}=s_{i} \forall i \in S_{k-1}\right\}=: P_{s}
$$

possuem um entrelaçador comum. 
Seja $k \in[m]$ e $s \in\{ \pm 1\}^{S_{k-1}}$. Note que $\left|P_{s}\right|=2$. Seja $e \in S_{k} \backslash S_{k-1}$. Sejam $r_{1}, r_{2} \in\{ \pm 1\}^{S_{k}}$ tais que $p_{r_{1}}, p_{r_{2}} \in P_{s}, r_{1}(e)=1$ e $r_{2}(e)=-1$. Pelo Teorema 4.23, temos que $P_{s}$ possui um entrelaçador comum se e somente se $\lambda p_{r_{1}}+(1-\lambda) p_{r_{2}}$ possui apenas raízes reais para todo $\lambda \in[0,1]$. Seja $\lambda \in[0,1]$ e seja $\mu: E \rightarrow[0,1]$ a função definida como

$$
\mu_{i}= \begin{cases}0 & \text { se } i \in S_{k-1} \text { e } s_{i}=-1 \\ 1 & \text { se } i \in S_{k-1} \text { e } s_{i}=1 \\ \lambda & \text { se } i \in S_{k} \backslash S_{k-1} \\ \frac{1}{2} & \text { se } i \in E \backslash S_{k}\end{cases}
$$

para todo $i \in E$. Defina $v_{S}:=2 \mathbb{1}_{S}-\mathbb{1}$ para todo $S \subseteq E$. Defina também $R_{1}:=\left\{i \in S_{k-1}: s_{i}=1\right\}$ e $R_{2}:=R_{1} \cup\left(S_{k} \backslash S_{k-1}\right)$. Por (5.5) e (5.6), temos que, para todo $S \subseteq E$, se existe $j \in S \cap S_{k-1}$ com $s_{j}=-1$ ou $j \in S_{k-1} \backslash S$ com $s_{j}=1$, então

$$
q_{S}(x):=\left(\prod_{j \in S} \mu_{j}\right)\left(\prod_{j \in E \backslash S}\left(1-\mu_{j}\right)\right) \operatorname{det}\left(x I-A_{G, v_{S}}\right)=0 .
$$

Assim, se $q_{S}(x) \neq 0$, então $S \cap S_{k-1}=R_{1}$. Logo, vale que

$$
\begin{aligned}
\sum_{S \subseteq E} & \left(\prod_{j \in S} \mu_{j}\right)\left(\prod_{j \in E \backslash S}\left(1-\mu_{j}\right)\right) \operatorname{det}\left(x I-A_{G, v_{S}}\right) \\
& =\sum_{S \subseteq E \backslash S_{k-1}}\left(\prod_{j \in S} \mu_{j}\right)\left(\prod_{j \in\left(E \backslash S_{k-1}\right) \backslash S}\left(1-\mu_{j}\right)\right) \operatorname{det}\left(x I-A_{G, v_{S} \cup R_{1}}\right) \\
& =\frac{1}{2^{m-k}} \sum_{S \subseteq E \backslash S_{k-1}}\left(\prod_{j \in S \cap S_{k}} \mu_{j}\right)\left(\prod_{j \in\left(\left(E \backslash S_{k-1}\right) \backslash S\right) \cap S_{k}}\left(1-\mu_{j}\right)\right) \operatorname{det}\left(x I-A_{G, v_{S} \cup R_{1}}\right) \operatorname{por}(5.8) \\
& =\frac{1}{2^{m-k}} \sum_{S \subseteq E \backslash S_{k-1}}\left(\prod_{j \in S \cap S_{k}} \lambda\right)\left(\prod_{j \in\left(\left(E \backslash S_{k-1}\right) \backslash S\right) \cap S_{k}}(1-\lambda)\right) \operatorname{det}\left(x I-A_{G, v_{S \cup R_{1}}}\right) \quad \text { por }(5.7) \\
& =\frac{1}{2^{m-k}}\left(\lambda \sum_{S \subseteq E \backslash S_{k}} \operatorname{det}\left(x I-A_{G, v_{S \cup R_{2}}}\right)+(1-\lambda) \sum_{S \subseteq E \backslash S_{k}} \operatorname{det}\left(x I-A_{G, v_{S \cup R_{1}}}\right)\right) \\
& =\frac{1}{2^{m-k}}\left(\lambda p_{r_{1}}+(1-\lambda) p_{r_{2}}\right) .
\end{aligned}
$$

Logo, pelo Teorema 5.23, temos que $\lambda p_{r_{1}}+(1-\lambda) p_{r_{2}}$ é real estável. Portanto, como esse polinômio é monovariado, então, pela Proposição 5.3, temos que todas as suas raízes são reais.

O segundo exemplo de árvores entrelaçadoras são árvores de polinômios associadas a variáveis aleatórias. Os polinômios folha dessas árvores são associados aos possíveis valores que as variáveis aleatórias em questão podem assumir. Assim, o número de folhas desse tipo de árvore de polinômios é o produto dos tamanhos dos suportes das variáveis aleatórias em questão. Segue a definição dos polinômios que compõe as folhas desse tipo de árvores entrelaçadoras.

Definição 5.31. Sejam $v_{1}, v_{2}, \ldots, v_{m}$ variáveis aleatórias que assumem valores em $\mathbb{C}^{n}$ com $R_{v_{i}}$ finito para todo $i \in[m]$. Defina $q_{v_{1}, v_{2}, \ldots, v_{m}}\left[w_{1}, w_{2}, \ldots, w_{m}\right] \in \mathbb{C}[x]$ como

$$
q_{v_{1}, v_{2}, \ldots, v_{m}}\left[w_{1}, w_{2}, \ldots, w_{m}\right](x):=\left(\prod_{i \in[m]} \mathbb{P}\left(v_{i}=w_{i}\right)\right) \operatorname{det}\left(x I-\sum_{i \in[m]} w_{i} w_{i}^{*}\right)
$$

para todo $\left(w_{1}, w_{2}, \ldots, w_{m}\right) \in R_{v_{1}} \times R_{v_{2}} \times \cdots \times R_{v_{m}}$. 
Sejam $v_{1}, v_{2}, \ldots, v_{m}$ variáveis aleatórias que assumem valores em $\mathbb{C}^{n}$ com $R_{v_{i}}$ finito para todo $i \in[m]$. Defina $R:=R_{v_{1}} \times R_{v_{2}} \times \cdots \times R_{v_{m}}$ e $A_{i}:=\mathbb{E}_{v_{i}} v_{i} v_{i}^{*}$ para todo $i \in[m]$. É fácil ver que

$$
\sum_{w \in R} q_{v_{1}, v_{2}, \ldots, v_{m}}\left[w_{1}, w_{2}, \ldots, w_{m}\right]=\mathbb{E}_{v_{i}, \ldots, v_{m}} \operatorname{det}\left(x I-\sum_{i \in[m]} v_{i} v_{i}^{*}\right) .
$$

Note que o lado direito da equação acima é uma representação do Mixed Characteristic Polynomial associado às matrizes $A_{1}, \ldots, A_{m}$. Assim, vale que os polinômios da família de polinômios $\left\{q_{v_{1}, \ldots, v_{m}}[w]\right\}_{w \in R}$ somam $\mu\left[A_{1}, \ldots, A_{m}\right]$.

Mostraremos, a seguir, que a família $\left\{q_{v_{1}, \ldots, v_{m}}[w]\right\}_{w \in R}$ forma uma árvore entrelaçadora de polinômios.

Teorema 5.32 (Marcus, Spielman e Srivastava [25]). Sejam $v_{1}, v_{2}, \ldots, v_{m}$ variáveis aleatórias que assumem valores em $\mathbb{C}^{n}$ com $R_{v_{i}}$ finito para todo $i \in[m]$. Então a família de polinômios $\left\{q_{v_{1}, \ldots, v_{m}}[w]\right\}_{w \in R_{v_{1}} \times R_{v_{2}} \times \cdots \times R_{v_{m}}}$ é uma família entrelaçadora de polinômios.

Demonstração. Defina $R_{i}:=R_{v_{i}} \times \cdots \times R_{v_{m}}$ para todo $i \in[m]$. Defina também

$$
q_{w_{1}, \ldots, w_{m}}:=q_{v_{1}, v_{2}, \ldots, v_{m}}\left[w_{1}, \ldots, w_{m}\right]
$$

para todo $\left(w_{1}, \ldots, w_{m}\right) \in R_{1} \mathrm{e}$

$$
q_{w_{1}, \ldots, w_{k}}:=\sum_{\left(r_{k+1}, \ldots, r_{m}\right) \in R_{k+1}} q_{v_{1}, v_{2}, \ldots, v_{m}}\left[w_{1}, \ldots, w_{k}, r_{k+1}, \ldots, r_{m}\right]
$$

para todo $k \in\{0, \ldots, m-1\}$ e todos $w_{1} \in R_{v_{1}}, \ldots, w_{k} \in R_{v_{k}}$. Defina também

$$
Q_{w_{1}, \ldots, w_{k}}:=\left\{q_{w_{1}, \ldots, w_{k}, r}: r \in R_{v_{k+1}}\right\} .
$$

para todo $k \in\{0, \ldots, m\}$ e todos $w_{1} \in R_{v_{1}}, \ldots, w_{k} \in R_{v_{k}}$.

Para provar que $\left\{q_{v_{1}, \ldots, v_{m}}[w]\right\}_{w \in R_{v_{1}} \times R_{v_{2}} \times \cdots \times R_{v_{m}}}$ é uma família entrelaçadora de polinômios, mostraremos que $Q_{w_{1}, \ldots, w_{k}}$ possui um entrelaçador comum para todo $k \in\{0, \ldots, m-1\}$ e todos $w_{1} \in R_{v_{1}}, \ldots, w_{k} \in R_{v_{k}}$.

Seja $k \in[m]$ e sejam $w_{1} \in R_{v_{1}}, \ldots, w_{k-1} \in R_{v_{k-1}}$. Seja $\lambda: R_{v_{k}} \rightarrow[0,1]$ uma função tal que $\sum_{r \in R_{v_{k}}} \lambda_{r}=1$. Note que

$$
\begin{aligned}
\sum_{r \in R_{v_{k}}} \lambda_{r} q_{w_{1}, \ldots, w_{k-1}, r} & =\sum_{w_{k} \in R_{v_{k}}}\left(\lambda_{w_{k}} \sum_{\left(w_{k+1}, \ldots, w_{m}\right) \in R_{k+1}} q_{w_{1}, \ldots, w_{m}}\right) \\
& =\sum_{w_{k} \in R_{v_{k}}}\left(\lambda_{w_{k}} \sum_{\left(w_{k+1}, \ldots, w_{m}\right) \in R_{k+1}}\left(\prod_{i \in[m]} \mathbb{P}\left(v_{i}=w_{i}\right)\right) \operatorname{det}\left(x I-\sum_{i \in[m]} w_{i} w_{i}^{*}\right)\right) \\
& =\sum_{\left(w_{k}, \ldots, w_{m}\right) \in R_{k}} \lambda_{w_{k}}\left(\prod_{i \in[m]} \mathbb{P}\left(v_{i}=w_{i}\right)\right) \operatorname{det}\left(x I-\sum_{i \in[m]} w_{i} w_{i}^{*}\right) \\
& =\left(\prod_{i \in[k-1]} \mathbb{P}\left(v_{i}=w_{i}\right)\right) \sum_{\left(w_{k}, \ldots, w_{m}\right) \in R_{k}} \lambda_{w_{k}}\left(\prod_{i=k}^{m} \mathbb{P}\left(v_{i}=w_{i}\right)\right) \operatorname{det}\left(x I-\sum_{i=k}^{m} w_{i} w_{i}^{*}\right) .
\end{aligned}
$$

Defina $\alpha:=\left(\prod_{i \in[k-1]} \mathbb{P}\left(v_{i}=w_{i}\right)\right)$ e $\beta:=\sum_{r \in R_{v_{k}}} \lambda_{r} \mathbb{P}\left(v_{k}=r\right)$. Como $\mathbb{P}\left(v_{k}=r\right)>0$ para todo $r \in R_{v_{k}}$, então $\beta>0$. Seja $u_{k}$ uma variável aleatória que assume valores em $\mathbb{C}^{n}$ tal que $R_{u_{k}}=R_{v_{k}}$ $\mathrm{e}$

$$
\mathbb{P}\left(u_{k}=r\right)=\frac{\lambda_{r} \mathbb{P}\left(v_{k}=r\right)}{\beta}
$$


para todo $r \in R_{u_{k}}$. Defina $R_{k}^{\prime}:=R_{u_{k}} \times R_{v_{k+1}} \times R_{v_{k+2}} \times \cdots \times R_{v_{m}}$. Defina também $A_{v}:=\mathbb{E}_{v} v v^{*}$ para todo $v \in\left\{u_{k}, v_{k+1}, v_{k+2}, \ldots, v_{m}\right\}$. Note que

$$
\begin{aligned}
\frac{1}{\beta} \sum_{r \in R_{v_{k}}} \lambda_{r} q_{w_{1}, \ldots, w_{k-1}, r} & =\alpha \sum_{\left(w_{k}, \ldots, w_{m}\right) \in R_{k}} \mathbb{P}\left(u_{k}=w_{k}\right)\left(\prod_{i=k+1}^{m} \mathbb{P}\left(v_{i}=w_{i}\right)\right) \operatorname{det}\left(x I-\sum_{i=k}^{m} w_{i} w_{i}^{*}\right) \\
& =\alpha \mathbb{E}_{u_{k}, v_{k+1}, \ldots, v_{m}} \operatorname{det}\left(x I-u_{k} u_{k}^{*}-\sum_{i=k+1}^{m} v_{i} v_{i}^{*}\right) \\
& =\alpha \mu\left[A_{u_{k}}, A_{v_{k+1}}, \ldots, A_{v_{m}}\right],
\end{aligned}
$$

onde a última igualdade vale pelo Corolário 5.29. Assim, pelo Lema 5.25, temos que

$$
\sum_{r \in R_{v_{k}}} \lambda_{r} q_{w_{1}, \ldots, w_{k-1}, r}
$$

possui somente raízes reais. Logo, pelo Teorema 4.23 , temos que $Q_{w_{1}, \ldots, w_{k}}$ possui um entrelaçador comum para todo $k \in\{0, \ldots, m-1\}$ e todos $w_{1} \in R_{v_{1}}, \ldots, w_{k} \in R_{v_{k}}$. Portanto, vale que $\left\{q_{v_{1}, \ldots, v_{m}}(w)\right\}_{w \in R_{m}}$ é uma árvore entrelaçadora de polinômios.

Partindo desse resultado, podemos utilizar a propriedade de raízes máximas das árvores entrelaçadoras de polinômios na família de polinômios $\left\{q_{v_{1}, \ldots, v_{m}}[w]\right\}_{w \in R}$. Assim, concluímos que as raízes de pelo menos um dos polinômios dessa família são limitadas superiormente pela raiz máxima da soma de todos os polinômios dessa família. Como mostrado anteriormente, a soma dessa família de polinômios é o Mixed Characteristic Polynomial associado às matrizes $A_{1}, \ldots, A_{m}$ e, portanto, a raiz máxima de $\mu\left[A_{1}, \ldots, A_{m}\right]$ limita superiormente todas as raízes de pelo menos um dos polinômios pertencentes a $\left\{q_{v_{1}, \ldots, v_{m}}[w]\right\}_{w \in R}$.

No Capítulo 8, apresentaremos um limitante superior para as raízes de $\mu\left[A_{1}, \ldots, A_{m}\right]$ que, por sua vez, também limitam as raízes de pelo menos um dos polinômios da família de polinômios $\left\{q_{v_{1}, \ldots, v_{m}}[w]\right\}_{w \in R}$. 


\section{Capítulo 6}

\section{Famílias infinitas de grafos de Ramanujan}

Neste capítulo, iremos provar a existência de famílias infinitas de grafos de Ramanujan que, como mostrado na Seção 3.3, são famílias de grafos expansores ótimos. Essa prova se deve a Marcus, Spielman e Srivastava [24], e se baseia nos conceitos de polinômios de emparelhamentos, árvores entrelaçadoras de polinômios e polinômios estáveis. Essas famílias são construídas iterativamente a partir de grafos bipartidos completos (que são grafos de Ramanujan) utilizando sucessivas construções de 2-lifts, que serão definidos na próxima seção.

Os 2-lifts são construções de grafos que recebem um grafo $G$ e sinais, positivos ou negativos, associados a cada aresta de $G$ e, a partir disso, geram um grafo $H$ com o dobro do número de vértices e arestas de $G$. Chamamos $H$ de 2-lift de $G$ independentemente dos sinais associados às arestas de $G$ escolhidos. Como cada aresta é associada a um sinal positivo ou negativo, qualquer grafo possui $2^{m}$ 2-lifts, onde $m$ é o número de arestas do grafo. A construção de 2-lifts também preserva regularidade, ou seja, se o grafo é regular, então todos os seus 2-lifts também são regulares de mesma valência. Além disso, todos os 2-lifts de um grafo bipartido também são bipartidos. Também vale que os autovalores das matrizes de adjacência dos 2-lifts de um grafo $G$ são a união dos autovalores de $A_{G}$ com novos autovalores, que variam de acordo com os sinais associados a cada aresta de $G$. Assim, para provar a existência de famílias infinitas de grafos expansores ótimos $d$-regulares, mostraremos que os novos autovalores da matriz de adjacência de pelo menos um dos 2-lifts de um grafo de Ramanujan $d$-regular são limitados superiormente por $2 \sqrt{d-1}$.

Associamos cada 2-lift de um grafo $G$ a uma matriz de sinais, definida no Capítulo 5 , referente aos sinais associados às arestas de $G$ e mostraremos que os autovalores dessas matrizes são iguais aos novos autovalores da matriz de adjacência de seu 2-lift associado. Além disso, no capítulo anterior, apresentamos alguns exemplos de árvores entrelaçadoras de polinômios, e um dos exemplos é a árvore formada pelos polinômios característicos das matrizes de sinais dos 2-lifts de qualquer grafo. Logo, podemos utilizar a propriedade de raízes máximas das árvores entrelaçadoras de polinômios para limitar superiormente os novos autovalores da matriz de adjacência de pelo menos um 2-lift do grafo original, onde o limitante é a raiz máxima da soma dos polinômios característicos dessas matrizes. Mostraremos, neste capítulo, que essa soma de polinômios característicos possui as mesmas raízes que o polinômio de emparelhamentos do grafo original, e caso esse grafo seja $d$-regular, o seu polinômio de emparelhamentos possui apenas raízes reais e sua raiz máxima é limitada superiormente por $2 \sqrt{d-1}$. Assim, os novos autovalores da matriz de adjacência de pelo menos um 2-lift de um grafo expansor ótimo são limitados por $2 \sqrt{d-1}$. Dessa forma, pelo menos um 2-lift de um grafo expansor ótimo também é um grafo expansor ótimo e, portanto, pelo menos uma das famílias 
formada por construções recursivas de 2-lifts a partir de um grafo bipartido completo é uma família infinita de grafos expansores ótimos. Como todos os 2-lifts de um grafo bipartido também são bipartidos, então todos os grafos pertencentes a essa família de grafos expansores ótimos $d$-regulares, que é construída a partir de grafos bipartidos completos, também são bipartidos. Logo, os valores absolutos de todos os autovalores não triviais (diferentes de $d$ ) das matrizes de adjacência dos grafos dessa família são limitados superiormente por $2 \sqrt{d-1}$ e, portanto, esses grafos são grafos de Ramanujan, e essa família de grafos expansores ótimos é também uma família de grafos de Ramanujan.

\section{$6.1 \quad 2$-lifts}

Um 2-lift de um grafo $G$ é um grafo $H$ com o dobro do número de vértices e arestas de $G$, de forma que metade dos vértices de $H$ pertence a um conjunto $V_{1}$ e a outra metade, a um conjunto $V_{2}$, satisfazendo as seguintes condições:

- todos os vértices de $G$ são mapeados em vértices de $V_{1}$ e $V_{2}$ por duas funções bijetoras $f_{1}$ e $f_{2}$, respectivamente,

- cada aresta $\{u, v\}$ de $G$ se expande em duas arestas de $H$, de forma que essas arestas são

internas: $\left\{f_{1}(u), f_{1}(v)\right\}$ e $\left\{f_{2}(u), f_{2}(v)\right\}$ ou

externas: $\left\{f_{1}(u), f_{2}(v)\right\}$ e $\left\{f_{2}(u), f_{1}(v)\right\}$.

Note que as arestas internas pertencem a $\left(\begin{array}{c}V_{1} \\ 2\end{array}\right) \cup\left(\begin{array}{c}V_{2} \\ 2\end{array}\right)$ e as arestas externas pertencem a $\delta_{H}\left(V_{1}\right)$. Note também que um único grafo possui vários 2-lifts e o que os diferencia é a escolha de expandir cada aresta em arestas internas ou externas. Apresentamos abaixo uma definição formal de 2-lifts.

Definição 6.1 (2-lifts). Seja $G=(V, E)$ um grafo. Dizemos que um grafo $H=(W, F)$ é um 2-lift de $G$ associado às funções $a$ e $\bar{a}$ se

- existe $U \subseteq W$ tal que $a: V \rightarrow U$ e $\bar{a}: V \rightarrow W \backslash U$ são bijetoras,

- $\operatorname{grau}_{G}(v)=\operatorname{grau}_{H}\left(a_{v}\right)=\operatorname{grau}_{H}\left(\bar{a}_{v}\right)$ para todo $v \in V$, e

- para todo $\{u, v\} \in E$ apenas uma das duas afirmativas vale

(i) $\left\{\left\{a_{u}, a_{v}\right\},\left\{\bar{a}_{u}, \bar{a}_{v}\right\}\right\} \subseteq F$ ou

(ii) $\left\{\left\{a_{u}, \bar{a}_{v}\right\},\left\{\bar{a}_{u}, a_{v}\right\}\right\} \subseteq F$.

Chamamos as arestas do tipo (i) de arestas internas, e as arestas do tipo (ii), de externas. Note que, se $H$ é um 2-lift de um grafo $G$ tal que todas as arestas de $H$ são do tipo (i), então $H$ é a união disjunta de dois grafos isomorfos a $G$. Chamamos esse 2-lift de 2-lift trivial de $G$. Seguem exemplos de 2-lifts do grafo bipartido completo $K_{3,3}$ e do grafo completo $K_{3}$.
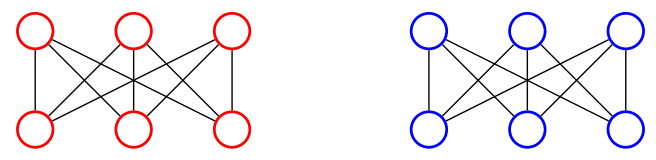

Figura 6.1: 2-lift trivial de $K_{3,3}$. 


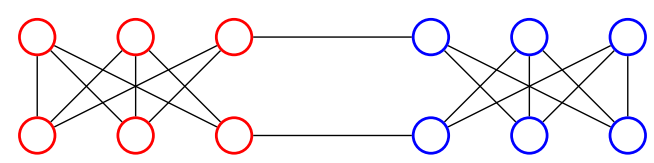

Figura 6.2: 2-lift de $K_{3,3}$.

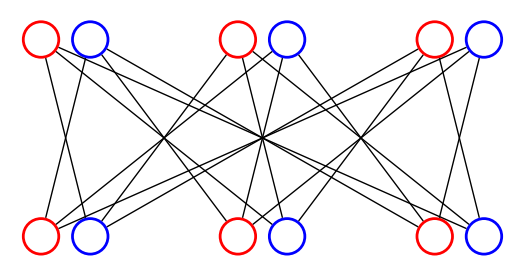

Figura 6.3: 2-lift de $K_{3,3}$.
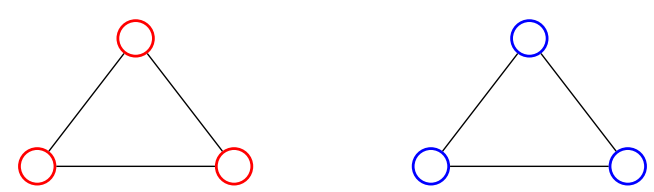

Figura 6.4: 2-lift trivial de $K_{3}$.

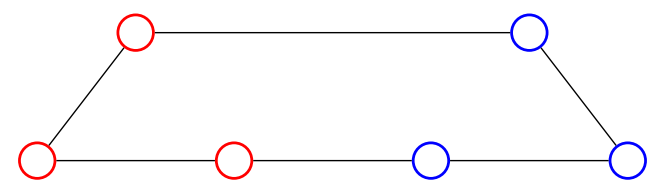

Figura 6.5: 2-lift de $K_{3}$.

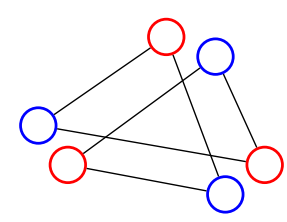

Figura 6.6: 2-lift de $K_{3}$. 
Note que os grafos representados nas Figuras 6.1 e 6.4 são 2-lifts triviais de $K_{3,3}$ e $K_{3}$, respectivamente. Note também que os 2-lifts representados nas Figuras 6.3 e 6.6 possuem apenas arestas do tipo (ii).

É fácil ver que os graus dos vértices de todos os 2-lifts de um grafo são iguais aos graus dos seus vértices associados do grafo original. Assim, se o grafo original é regular, todos os 2-lifts desse grafo também são regulares de mesma valência. Também vale que, caso o grafo original seja bipartido, todos os 2-lifts desse grafo também são bipartidos. Além disso, se o grafo original é $(c, d)$-birregular, então todos os 2-lifts desse grafo também são $(c, d)$-birregulares e possuem a mesma cobertura universal do grafo original.

Proposição 6.2. Seja $G$ um grafo $d$-regular e $H$ um 2-lift de $G$. Então $H$ é $d$-regular.

Demonstração. Suponha que $H$ é um 2-lift de $G$ associado às funções a e $\bar{a}$. Pela Definição 6.1, temos que

$$
\operatorname{grau}_{G}(v)=\operatorname{grau}_{H}\left(a_{v}\right)=\operatorname{grau}_{H}\left(\bar{a}_{v}\right)
$$

para todo $v \in V(G)$. Note que $a$ e $\bar{a}$ são bijetoras e que $\operatorname{Im}(a) \cap \operatorname{Im}(\bar{a})=\varnothing$. Portanto, como $|V(H)|=2|V(G)|$, então $\operatorname{grau}_{H}(v)=d$ para todo $v \in V(H)$.

Proposição 6.3. Seja $G$ um grafo bipartido e $H$ um 2-lift de $G$. Então $H$ é bipartido.

Demonstração. Suponha que $H$ é um 2-lift de $G$ associado às funções $a$ e $\bar{a}$. Sejam $U, V \subseteq V(G)$ tais que $E(G) \cap\left(\begin{array}{c}U \\ 2\end{array}\right)=\varnothing$ e $E(G) \cap\left(\begin{array}{c}V \\ 2\end{array}\right)=\varnothing$. Defina $U^{\prime}:=a(U) \cup \bar{a}(U)$ e $V^{\prime}:=a(V) \cup \bar{a}(V)$. Seja $e \in E(H)$. Note que existe $\{u, v\} \in E(G)$, com $u \in U$ e $v \in V$, tais que

- $a_{u} \in e$ e $a_{v} \in e$ ou

- $\bar{a}_{u} \in e$ e $\bar{a}_{v} \in e$ ou

- $a_{u} \in e$ e $\bar{a}_{v} \in e$ ou

- $\bar{a}_{u} \in e$ e $a_{v} \in e$.

Assim, como $u \in U$ e $v \in V$, então $a_{u}, \bar{a}_{u} \in U^{\prime}$ e $a_{v}, \bar{a}_{v} \in V^{\prime}$. Portanto, vale que $e \cap U^{\prime} \neq \varnothing$ e $e \cap V^{\prime} \neq \varnothing$.

Proposição 6.4. Seja $G$ um grafo $(c, d)$-birregular e $H$ um 2-lift de $G$. Então $H$ é $(c, d)$-birregular.

Demonstração. Suponha que $H$ é um 2-lift de $G$ associado às funções $a$ e $\bar{a}$. Pela Proposição 6.3, temos que $H$ é bipartido. Sejam $U, V \subseteq V(G)$ tais que

$$
E(G) \cap\left(\begin{array}{l}
U \\
2
\end{array}\right)=\varnothing \quad \text { e } \quad E(G) \cap\left(\begin{array}{l}
V \\
2
\end{array}\right)=\varnothing
$$

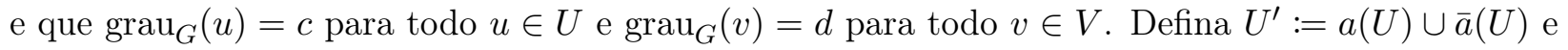
$V^{\prime}:=a(V) \cup \bar{a}(V)$. Note que

$$
E(H) \cap\left(\begin{array}{c}
U^{\prime} \\
2
\end{array}\right)=\varnothing \quad \text { e } \quad E(H) \cap\left(\begin{array}{c}
V^{\prime} \\
2
\end{array}\right)=\varnothing .
$$

Pela Definição 6.1, temos que $\operatorname{grau}_{G}(v)=\operatorname{grau}_{H}\left(a_{v}\right)=\operatorname{grau}_{H}\left(\bar{a}_{v}\right)$ para todo $v \in V(G)$. Logo, vale que $\operatorname{grau}_{H}(u)=c$ para todo $u \in U^{\prime}$ e $\operatorname{grau}_{H}(v)=d$ para todo $v \in V^{\prime}$. Portanto, o grafo $H$ é $(c, d)$-birregular. 
Proposição 6.5. Sejam $G, H$ grafos $(c, d)$-birregulares. Então as coberturas universais $T_{G}$ e $T_{H}$ são isomorfas.

Demonstração. Sejam $U_{G}, V_{G} \subseteq V(G) \operatorname{com} U_{G} \cup V_{G}=V(G)$ e $U_{H}, V_{H} \subseteq V(H)$ com $U_{H} \cup V_{H}=$ $V(H)$ tais que $\operatorname{grau}\left(u_{G}\right)=\operatorname{grau}\left(u_{H}\right)=c$ para todos $u_{G} \in U_{G}$ e $u_{H} \in U_{H}$ e $\operatorname{grau}\left(v_{G}\right)=\operatorname{grau}\left(v_{H}\right)=d$ para todos $v_{G} \in V_{G}$ e $v_{H} \in V_{H}$. Sejam

$$
g_{u}:\left[\operatorname{grau}_{G}(u)\right] \rightarrow \Gamma_{G}(u) \quad \text { e } \quad h_{v}:\left[\operatorname{grau}_{H}(v)\right] \rightarrow \Gamma_{H}(v)
$$

funções bijetoras para todos $u \in V(G)$ e $v \in V(H)$.

Sejam $u_{G} \in U_{G}$ e $u_{H} \in U_{H}$. Defina

$$
A_{m}:=\left\{a \in \mathbb{N}^{m}: a_{i} \in[c] \text { se } i \text { é ímpar e } a_{i} \in[d] \text { se } i \text { é par }\right\}
$$

para todo $m \in \mathbb{N}$. Seja $m \in \mathbb{N}$. Para todo $a \in A_{m}$, defina $p_{G}(a)$ como o passeio

$$
\left(u_{0}, u_{1}, \ldots, u_{m}\right),
$$

onde $u_{0}=u_{G}$ e $u_{i}:=g_{u_{i-1}}\left(a_{i}\right)$ para todo $i \in[m]$. Defina também $p_{H}(a)$ para todo $a \in A_{m}$ como o passeio

$$
\left(u_{0}, u_{1}, \ldots, u_{m}\right)
$$

onde $u_{0}=u_{H}$ e $u_{i}:=h_{u_{i-1}}\left(a_{i}\right)$ para todo $i \in[m]$.

Note que

$$
V\left(T_{G}\right)=\bigcup_{m \in \mathbb{N}} p_{G}\left(A_{m}\right) \quad \text { e } \quad V\left(T_{H}\right)=\bigcup_{m \in \mathbb{N}} p_{H}\left(A_{m}\right) .
$$

Seja $f: V\left(T_{G}\right) \rightarrow V\left(T_{H}\right)$ uma função bijetora definida como $f\left(p_{G}(a)\right)=p_{H}(a)$ para todo $a \in$ $\bigcup_{m \in \mathbb{N}} A_{m}$. Sejam $u_{1}, v_{1} \in V\left(T_{G}\right)$ e $u_{2}, v_{2} \in V\left(T_{H}\right)$. Pela definição de cobertura universal, temos que

$$
\begin{aligned}
& \left\{u_{1}, v_{1}\right\} \in E\left(T_{G}\right) \Longleftrightarrow u_{1} \in P_{G, v_{1}} \text { ou } v_{1} \in P_{G, u_{1}}, \\
& \left\{u_{2}, v_{2}\right\} \in E\left(T_{H}\right) \Longleftrightarrow u_{2} \in P_{H, v_{2}} \text { ou } v_{2} \in P_{H, u_{2}} .
\end{aligned}
$$

Note que $u_{1} \in P_{G, v_{1}}$ se e somente se $f\left(u_{1}\right) \in P_{H, f\left(v_{1}\right)}$, o que implica que $\left\{u_{1}, v_{1}\right\} \in E\left(T_{G}\right)$ se e somente se $\left\{f\left(u_{1}\right), f\left(v_{1}\right)\right\} \in E\left(T_{H}\right)$. Portanto, vale que $T_{G}$ é isomorfo a $T_{H}$.

Proposição 6.6. Seja $G$ um grafo birregular e $H$ um 2-lift de $G$. Então as coberturas universais $T_{G}$ e $T_{H}$ são isomorfas.

Demonstração. O resultado segue das Proposições 6.4 e 6.5.

Para entender o comportamento espectral dos 2-lifts de um grafo, definiremos as funções de sinais a seguir.

Definição 6.7. Sejam $G=(V, E)$ um grafo e $H$ um 2-lift de $G$ associado às funções $a$ e $\bar{a}$. Defina a função de sinais $s: E \rightarrow\{ \pm 1\}$ do 2-lift $H$ de $G$ como

$$
s(\{u, v\}):= \begin{cases}+1, & \text { se }\left\{\left\{a_{u}, a_{v}\right\},\left\{\bar{a}_{u}, \bar{a}_{v}\right\}\right\} \subseteq E(H), \\ -1, & \text { se }\left\{\left\{a_{u}, \bar{a}_{v}\right\},\left\{\bar{a}_{u}, a_{v}\right\}\right\} \subseteq E(H),\end{cases}
$$

para todo $\{u, v\} \in E$. 
Utilizamos a função de sinais em conjunto com as matrizes da Definição 5.22 , denotando $A_{G, s}$ por matriz de sinais do 2-lift $H$ de $G$, onde $s$ é a função de sinais do 2-lift $H$ de $G$.

Considere o grafo $H_{1}$ como o 2-lift trivial de $K_{3}$, e $H_{2}$ e $H_{3}$ como os 2-lifts de $K_{3}$ representados pelas Figuras 6.5 e 6.6, respectivamente. Sejam $s_{1}, s_{2}$ e $s_{3}$ as funções de sinais dos 2-lifts $H_{1}, H_{2}$ e $H_{3}$ de $K_{3}$, respectivamente. Note que $\operatorname{Im}\left(s_{1}\right)=\{+1\}$ e que $\operatorname{Im}\left(s_{3}\right)=\{-1\}$. Note também que

$$
A_{K_{3}, s_{1}}=\left[\begin{array}{ccc}
0 & +1 & +1 \\
+1 & 0 & +1 \\
+1 & +1 & 0
\end{array}\right], \quad A_{K_{3}, s_{2}}=\left[\begin{array}{ccc}
0 & -1 & +1 \\
-1 & 0 & +1 \\
+1 & +1 & 0
\end{array}\right], \quad A_{K_{3}, s_{3}}=\left[\begin{array}{ccc}
0 & -1 & -1 \\
-1 & 0 & -1 \\
-1 & -1 & 0
\end{array}\right]
$$

Assim, vale que

- $(1,0,-1)^{\top},(1,-2,1)^{\top},(1,1,1)^{\top}$ são autovetores de $A_{K_{3}, s_{1}}$ associados aos autovalores $-1,-1,2$, respectivamente,

- $(1,1,-1)^{\top},(0,1,1)^{\top},(2,-1,1)^{\top}$ são autovetores de $A_{K_{3}, s_{2}}$ associados aos autovalores $-2,1,1$, respectivamente,

- $(1,1,1)^{\top},(1,-2,1)^{\top},(1,0,-1)^{\top}$ são autovetores de $A_{K_{3}, s_{3}}$ associados aos autovalores $-2,1,1$, respectivamente.

O resultado abaixo mostra que os autovalores da matriz de adjacência dos 2-lifts de um grafo são a união dos autovalores do grafo original com os autovalores da matriz de sinais do 2-lift em questão.

Teorema 6.8 (Bilu e Linial [4]). Sejam $G$ um grafo e $H$ um 2-lift de $G$. Seja $s$ a função de sinais do 2-lift $H$ de $G$. Então

$$
\operatorname{Spec}\left(A_{H}\right)=\operatorname{Spec}\left(A_{G}\right) \cup \operatorname{Spec}\left(A_{G, s}\right),
$$

preservando a multiplicidade geométrica dos autovalores.

Demonstração. Seja $n$ o número de vértices de $G$. Suponha que $H$ é um 2-lift de $G$ associado às funções $a$ e $\bar{a}$. Denote $U:=\operatorname{Im}(a)$ e $V:=\operatorname{Im}(\bar{a})$. Note que $A_{H}[U, U]=A_{H}[V, V]=: A$ e $A_{H}[U, V]=A_{H}[V, U]=: B$. Assim, vale que

$$
A_{H}=\left[\begin{array}{ll}
A & B \\
B & A
\end{array}\right]
$$

Note também que $A+B=A_{G}$ e $A-B=A_{G, s}$.

Seja $v$ um autovetor de $A_{G}$. Note que existe $i \in[n]$ tal que $(A+B) v=\lambda_{i}^{\downarrow}\left(A_{G}\right) v$, o que implica que $A v+B v=\lambda_{i}^{\downarrow}\left(A_{G}\right) v$. Assim,

$$
A_{H}[U, U \cup V](v \oplus v)=A_{H}[V, U \cup V](v \oplus v)=\lambda_{i}^{\downarrow}\left(A_{G}\right) v .
$$

Logo, $A_{H}(v \oplus v)=\lambda_{i}^{\downarrow}\left(A_{G}\right)(v \oplus v)$. Portanto, $\lambda_{i}^{\downarrow}\left(A_{G}\right) \in \operatorname{Spec}\left(A_{H}\right)$ para todo $i \in[n]$.

Seja $v$ um autovetor de $A_{G, s}$. Note que existe $i \in[n]$ tal que $(A-B) v=\lambda_{i}^{\downarrow}\left(A_{G, s}\right) v$. Assim,

$$
A_{H}[U, U \cup V](v \oplus-v)=-A_{H}[V, U \cup V](v \oplus-v)=\lambda_{i}^{\downarrow}\left(A_{G, s}\right) v .
$$

Logo, $A_{H}(v \oplus-v)=\lambda_{i}^{\downarrow}\left(A_{G}\right)(v \oplus-v)$. Portanto, $\lambda_{i}^{\downarrow}\left(A_{G, s}\right) \in \operatorname{Spec}\left(A_{H}\right)$ para todo $i \in[n]$. 
Sejam $u_{1}, u_{2}, \ldots, u_{n}$ autovetores ortogonais de $A_{G}$ e $v_{1}, v_{2}, \ldots, v_{n}$ autovetores ortogonais de $A_{G, s}$. Como mostrado anteriormente,

$$
u_{1} \oplus u_{1}, u_{2} \oplus u_{2}, \ldots, u_{n} \oplus u_{n}, v_{1} \oplus-v_{1}, v_{2} \oplus-v_{2}, \ldots, v_{n} \oplus-v_{n}
$$

são autovetores de $A_{H}$. Note que esses autovetores são dois a dois ortogonais. Portanto, vale que

$$
\operatorname{Spec}\left(A_{H}\right)=\operatorname{Spec}\left(A_{G}\right) \cup \operatorname{Spec}\left(A_{G, s}\right),
$$

preservando a multiplicidade geométrica dos autovalores.

Note que no Teorema 6.8 fazemos uso de operações e relações sobre matrizes e vetores indexados por conjuntos distintos. Considere que os índices foram padronizados em todas essas ocorrências.

Considere novamente os grafos $H_{1}, H_{2}$ e $H_{3}$ como os 2-lifts de $K_{3}$ representados pelas Figuras $6.4,6.5$ e 6.6 , respectivamente. Note que

$$
A_{H_{1}}=\left[\begin{array}{llllll}
0 & 1 & 1 & 0 & 0 & 0 \\
1 & 0 & 1 & 0 & 0 & 0 \\
1 & 1 & 0 & 0 & 0 & 0 \\
0 & 0 & 0 & 0 & 1 & 1 \\
0 & 0 & 0 & 1 & 0 & 1 \\
0 & 0 & 0 & 1 & 1 & 0
\end{array}\right], \quad A_{H_{2}}=\left[\begin{array}{cccccc}
0 & 0 & 1 & 0 & 1 & 0 \\
0 & 0 & 1 & 1 & 0 & 0 \\
1 & 1 & 0 & 0 & 0 & 0 \\
0 & 1 & 0 & 0 & 0 & 1 \\
1 & 0 & 0 & 0 & 0 & 1 \\
0 & 0 & 0 & 1 & 1 & 0
\end{array}\right], \quad A_{H_{3}}=\left[\begin{array}{cccccc}
0 & 0 & 0 & 0 & 1 & 1 \\
0 & 0 & 0 & 1 & 0 & 1 \\
0 & 0 & 0 & 1 & 1 & 0 \\
0 & 1 & 1 & 0 & 0 & 0 \\
1 & 0 & 1 & 0 & 0 & 0 \\
1 & 1 & 0 & 0 & 0 & 0
\end{array}\right] .
$$

Assim, vale que

- $(1,0-1,1,0,-1)^{\top},(1,-2,1,1,-2,1)^{\top},(1,1,1,1,1,1)^{\top}$ são autovetores de $H_{1}, H_{2}$ e $H_{3}$ associados aos autovalores $-1,-1,2$, respectivamente,

- $(1,0,-1,-1,0,1)^{\top},(1,-2,1,-1,2,-1)^{\top},(1,1,1,-1,-1,-1)^{\top}$ são autovetores de $H_{1}$ associados aos autovalores $-1,-1,2$, respectivamente,

- $(1,1,-1,-1,-1,1)^{\top},(0,1,1,0,-1,-1)^{\top},(2,-1,1,-2,1,-1)^{\top}$ são autovetores de $H_{2}$ associados aos autovalores $-2,-1,1$, respectivamente,

- $(1,1,1,-1,-1,-1)^{\top},(1,-2,1,-1,2,-1)^{\top},(1,0,-1,-1,0,1)^{\top}$ são autovetores de $H_{3}$ associados aos autovalores $-2,1,1$, respectivamente,

Note que $(1,0,-1)^{\top},(1,-2,1)^{\top},(1,1,1)^{\top}$ são autovetores de $K_{3}$ associados, respectivamente, aos autovalores $-1,-1,2$. Logo, vale que $\operatorname{Spec}\left(A_{H_{i}}\right)=\operatorname{Spec}\left(A_{K_{3}}\right) \cup \operatorname{Spec}\left(A_{K_{3}, s_{i}}\right)$ para todo $i \in[3]$, onde $s_{1}, s_{2}$ e $s_{3}$ são as funções de sinais dos 2-lifts $H_{1}, H_{2}$ e $H_{3}$ de $K_{3}$, respectivamente.

\subsection{Polinômios de emparelhamentos}

Nesta seção, mostraremos outras propriedades de polinômios de emparelhamentos que serão utilizadas na prova de existência de famílias infinitas de grafos de Ramanujan:

- o polinômio de emparelhamentos de um grafo é igual ao polinômio característico esperado da matriz de sinais de um 2-lift aleatório desse grafo,

- as raízes dos polinômios de emparelhamentos são reais, 
- caso o grafo seja $d$-regular, as raízes de seu polinômio de emparelhamentos são limitadas por $2 \sqrt{d-1}$.

Como mostrado no Capítulo 5, os polinômios característicos das matrizes de sinais dos 2-lifts de um grafo formam uma árvore entrelaçadora de polinômios. Essa propriedade de 2-lifts, juntamente com os resultados de polinômios de emparelhamentos, será utilizada para limitar superiormente os autovalores da matriz de sinais de pelo menos um 2-lift do grafo original por $2 \sqrt{d-1}$, onde $d$ é a valência do grafo original.

A primeira propriedade de polinômios de emparelhamentos citada anteriormente foi provada por Godsil e Gutman [11], e será mostrada a seguir. As demais propriedades serão mostradas na próxima seção.

Teorema 6.9 ([12, Corolário 2.2]). Seja $G=(V, E)$ um grafo. Então,

$$
\mu_{G}(x)=\mathbb{E}_{s \in\{ \pm 1\}^{E}}\left(\operatorname{det}\left(x I-A_{G, s}\right)\right),
$$

onde a variável aleatória $s \in\{ \pm 1\}^{E}$ é escolhida de acordo com a distribuição uniforme.

Demonstração. Defina $n:=|V|$. Seja $s \in\{ \pm 1\}^{E}$. Seja $S_{V}:=\{\pi: V \rightarrow V: \pi$ é bijetora $\}$ e seja $\operatorname{sgn}(\pi)$ o sinal da permutação $\pi$ para todo $\pi \in S_{V}$. Note que

$$
\begin{aligned}
\operatorname{det}\left(x I-A_{G, s}\right) & =\sum_{\pi \in S_{V}} \operatorname{sgn}(\pi) \prod_{i \in V}\left(x I-A_{G, s}\right)_{i, \pi(i)} \\
& =\sum_{\pi \in S_{V}} \operatorname{sgn}(\pi) \prod_{i \in V}\left((x I)_{i, \pi(i)}-\left(A_{G, s}\right)_{i, \pi(i)}\right) .
\end{aligned}
$$

Defina $U_{\pi}:=\{i \in V: \pi(i) \neq i\}$ para todo $\pi \in S_{V}$. Defina também $C_{m}:=\left\{\pi \in S_{V}:\left|U_{\pi}\right|=m\right\}$ para todo $m \in\{0,1, \ldots, n\}$. Assim, vale que $\bigcup_{m \in\{0, \ldots, n\}} C_{m}=S_{V}$. Então

$$
\operatorname{det}\left(x I-A_{G, s}\right)=\sum_{m=0}^{n} \sum_{\pi \in C_{m}} \operatorname{sgn}(\pi) \prod_{i \in V}\left((x I)_{i, \pi(i)}-\left(A_{G, s}\right)_{i, \pi(i)}\right) .
$$

Sejam $m \in\{0, \ldots, n\}$ e $\pi \in C_{m}$ e seja $i \in V$. Como a diagonal da matriz $A_{G, s}$ é 0, então, se $\pi(i)=i$, vale que $\left(A_{G, s}\right)_{i, \pi(i)}=0$. Assim, vale que

$$
\prod_{i \in V}\left((x I)_{i, \pi(i)}-\left(A_{G, s}\right)_{i, \pi(i)}\right)=(x-0)^{n-m} \prod_{i \in U_{\pi}}\left(-\left(A_{G, s}\right)_{i, \pi(i)}\right) .
$$

Note que

$$
A_{G, s}(i, j)= \begin{cases}s_{i, j} & \text { se }\{i, j\} \in E, \\ 0 & \text { se }\{i, j\} \notin E,\end{cases}
$$

para todos $i, j \in V$. Defina $C_{m}^{\prime}:=\left\{\pi \in C_{m}:\left\{i, \pi_{i}\right\} \in E \forall i \in V\right\}$ para todo $m \in\{0,1 \ldots, n\}$. Logo, vale que

$$
\operatorname{det}\left(x I-A_{G, s}\right)=\sum_{m=0}^{n} x^{n-m} \sum_{\pi \in C_{m}^{\prime}} \operatorname{sgn}(\pi) \prod_{i \in U_{\pi}}\left(-s_{\{i, \pi(i)\}}\right) .
$$


Portanto, vale que

$$
\mathbb{E}_{s \in\{ \pm 1\}^{E}}\left(\operatorname{det}\left(x I-A_{G, s}\right)\right)=\sum_{m=0}^{n} x^{n-m} \sum_{\pi \in C_{m}^{\prime}} \operatorname{sgn}(\pi) \mathbb{E}_{s \in\{ \pm 1\}^{E}}\left(\prod_{i \in U_{\pi}}\left(-s_{\{i, \pi(i)\}}\right)\right) .
$$

Denote $\bar{C}_{m}:=\left\{\pi \in C_{m}^{\prime}: \pi(\pi(i))=i \forall i \in V\right\}$ para todo $m \in\{0,1, \ldots, n\}$. Seja $m \in$ $\{0,1, \ldots, n\}$. Sejam $\pi \in C_{m}^{\prime} \operatorname{com} \pi \notin \bar{C}_{m}$ e $k \in V$ tal que

$$
\pi(\pi(k)) \neq k \text {. }
$$

$\operatorname{Logo}$, como $\mathbb{E}_{s \in\{ \pm 1\}^{E}}\left(-s_{k, \pi(k)}\right)=0$, então

$$
\mathbb{E}_{s \in\{ \pm 1\}^{E}}\left(\prod_{i \in U_{\pi}}\left(-s_{\{i, \pi(i)\}}\right)\right)=\mathbb{E}_{s \in\{ \pm 1\}^{E}}\left(-s_{k, \pi(k)}\right) \mathbb{E}_{s \in\{ \pm 1\}^{E}}\left(\prod_{i \in U_{\pi} \backslash\{k\}}\left(-s_{\{i, \pi(i)\}}\right)\right)=0 .
$$

Assim, vale que

$$
\mathbb{E}_{s \in\{ \pm 1\}^{E}}\left(\operatorname{det}\left(x I-A_{G, s}\right)\right)=\sum_{m=0}^{n} x^{n-m} \sum_{\pi \in \bar{C}_{m}} \operatorname{sgn}(\pi) \mathbb{E}_{s \in\{ \pm 1\}^{E}}\left(\prod_{i \in U_{\pi}}\left(-s_{\{i, \pi(i)\}}\right)\right) .
$$

Sejam $\pi \in \bar{C}_{m}$ e $U \subsetneq U_{\pi}$ tal que $\pi(U)=U_{\pi} \backslash U$. Como $\mathbb{E}\left(s_{i, j}^{2}\right)=1$, então

$$
\mathbb{E}_{s \in\{ \pm 1\}^{E}}\left(\prod_{i \in U_{\pi}}\left(-s_{\{i, \pi(i)\}}\right)\right)=\mathbb{E}_{s \in\{ \pm 1\}^{E}}\left(\prod_{i \in U}\left(s_{\{i, \pi(i)\}}^{2}\right)\right)=1
$$

Note que $\operatorname{sgn}(\pi)=(-1)^{\frac{m}{2}}$ para todo $\pi \in \bar{C}_{m}$. Note também que $\{\{i, \pi(i)\}: i \in V\}$ é um emparelhamento em $G$ para todo $\bar{C}_{m}$ e todo $\pi \in \bar{C}_{m}$. Além disso, se $m$ é ímpar, então $\bar{C}_{m}=\varnothing$. Portanto,

$$
\begin{aligned}
\mathbb{E}_{s \in\{ \pm 1\}^{E}}\left(\operatorname{det}\left(x I-A_{G, s}\right)\right) & =\sum_{m=0}^{n} x^{n-m} \sum_{\pi \in \bar{C}_{m}}(-1)^{\frac{m}{2}} \\
& =\sum_{m=0}^{n} x^{n-m}(-1)^{\frac{m}{2}} \mathrm{~m}_{G}\left(\frac{m}{2}\right) \\
& =\sum_{m \in \mathbb{N}} x^{n-2 m}(-1)^{m} \mathrm{~m}_{G}(m) \\
& =\mu_{G}(x),
\end{aligned}
$$

onde $\mathrm{m}_{G}(m)$ é o número de emparelhamentos em $G$ com $m$ arestas.

Considere o grafo completo $K_{3}$. Denote $E:=E\left(K_{3}\right)$. Seja $p_{s} \in \mathbb{R}[x]$ definido como $p_{s}(x):=$ $\operatorname{det}\left(x I-A_{K_{3}, s}\right)$ para todo $s \in\{ \pm 1\}^{E}$. Note que

$$
\begin{aligned}
& p_{(+1,+1,+1)}(x)=p_{(+1,-1,-1)}(x)=p_{(-1,+1,-1)}(x)=p_{(-1,-1,+1)}(x)=x^{3}-3 x-2, \\
& p_{(-1,-1,-1)}(x)=p_{(-1,+1,+1)}(x)=p_{(+1,-1,+1)}(x)=p_{(+1,+1,-1)}(x)=x^{3}-3 x+2 .
\end{aligned}
$$

Logo, vale que

$$
\mathbb{E}_{s \in\{ \pm 1\}^{E}}\left(\operatorname{det}\left(x I-A_{K_{3}, s}\right)\right)=\frac{x^{3}-3 x-2}{2}+\frac{x^{3}-3 x+2}{2}=x^{3}-3 x=\mu_{K_{3}}(x) .
$$




\subsubsection{Raízes de polinômios de emparelhamentos}

Combinando o Teorema 6.9 com um exemplo de polinômios reais estáveis mostrado no Teorema 5.23, é fácil ver que polinômios de emparelhamentos possuem somente raízes reais. Seja $G=(V, E)$ um grafo. Defina $v_{S}:=2 \mathbb{1}_{S}-\mathbb{1}$ para todo $S \subseteq E$. Pelo resultado mostrado na seção anterior, temos que

$$
\mu_{G}(x)=\mathbb{E}_{s \in\{ \pm 1\}^{E}}\left(\operatorname{det}\left(x I-A_{G, s}\right)\right)=\sum_{S \subseteq E}\left(\prod_{j \in S} \lambda_{j}\right)\left(\prod_{j \in E \backslash S}\left(1-\lambda_{j}\right)\right) \operatorname{det}\left(x I-A_{G, v_{S}}\right) .
$$

Como mostrado no capítulo de polinômios estáveis, o polinômio do lado direito é real estável. Logo, os polinômios de emparelhamentos também são reais estáveis e, como estes são monovariados, possuem somente raízes reais.

Para mostrar que polinômios de emparelhamentos possuem somente raízes reais, sem utilizar as técnicas de polinômios estáveis apresentadas no Capítulo 5, mostramos primeiramente que todas as raízes dos polinômios de emparelhamentos de grafos completos com pesos positivos nas arestas são reais. Dessa forma, utilizamos, mais adiante, um argumento de continuidade para mostrar que todas as raízes dos polinômios de emparelhamentos de qualquer grafo com pesos não negativos nas arestas são reais.

Teorema 6.10 (vide [16]). Seja $G=\left(V,\left(\begin{array}{c}V \\ 2\end{array}\right)\right)$ um grafo com $|V| \geq 1$ e $w:\left(\begin{array}{c}V \\ 2\end{array}\right) \rightarrow \mathbb{R}_{++}$. Então, o polinômio $\mu_{G, \boldsymbol{w}}$ possui apenas raízes reais.

Demonstração. Defina $n:=|V|$. Mostraremos que, para todo $v \in V$, vale que $\mu_{G, \boldsymbol{w}}$ e $\mu_{G-v, \boldsymbol{w}}$ possuem apenas raízes reais e, além disso, $\mu_{G-v, \boldsymbol{w}}$ entrelaça estritamente o polinômio $\mu_{G, \boldsymbol{w}}$. Provaremos esse resultado por indução em $n$.

Suponha que $n=2$. Como $G$ é completo, então $\mu_{G, \boldsymbol{w}}=x^{2}-w$ e $\mu_{G-v, \boldsymbol{w}}=x$ para todo $v \in V$. Assim, para todo $v \in V$, vale que as raízes de $\mu_{G, \boldsymbol{w}}$ e $\mu_{G-v, \boldsymbol{w}}$ são reais e $\mu_{G-v, \boldsymbol{w}}$ entrelaça estritamente $\mu_{G, \boldsymbol{w}}$.

Suponha que $n>2$. Seja $v \in V$. Defina

$$
q:=\sum_{u \in \Gamma_{G}(v)} w(\{u, v\}) \mu_{G-v-u, \boldsymbol{w}} .
$$

Pelo Teorema 2.61, temos que $\mu_{G, \boldsymbol{w}}(x)=x \mu_{G-v, \boldsymbol{w}}(x)-q(x)$. Pela hipótese de indução, o polinômio $\mu_{G-v-u, \boldsymbol{w}}$ entrelaça estritamente o polinômio $\mu_{G-v, \boldsymbol{w}}$ para todo $u \in \Gamma_{G}(v)$. Logo, pelo Lema 4.9, temos que $q$ entrelaça estritamente $\mu_{G-v, \boldsymbol{w}}$.

Sejam $\alpha_{1}, \alpha_{2}, \ldots, \alpha_{n-1}$ as raízes de $\mu_{G-v, \boldsymbol{w}}$ e $\beta_{1}, \beta_{2}, \ldots, \beta_{n-2}$ as raízes de $q$ tais que

$$
\alpha_{1}<\beta_{1}<\alpha_{2}<\beta_{2}<\cdots<\beta_{n-2}<\alpha_{n-1} .
$$

Como $q$ tem coeficiente dominante positivo e entrelaça estritamente $\mu_{G-v, \boldsymbol{w}}$, vale que

$$
q\left(\alpha_{n-1-k}\right)>0
$$

para todo $k \in\{0, \ldots, n-2\}$ par e

$$
q\left(\alpha_{n-1-k}\right)<0
$$

para todo $k \in\{0, \ldots, n-2\}$ ímpar. Note que $\mu_{G, \boldsymbol{w}}\left(\alpha_{k}\right)=0-q\left(\alpha_{k}\right)$ para todo $k \in[n-1]$. Assim, $\mu_{G, \boldsymbol{w}}\left(\alpha_{n-1-k}\right)<0$ para todo $k \in\{0, \ldots, n-2\}$ par e $\mu_{G, \boldsymbol{w}}\left(\alpha_{n-1-k}\right)<0$ para todo $k \in\{0, \ldots, n-2\}$ ímpar. Portanto, para todo $k \in[n-2]$, existe $y \in\left(\alpha_{k-1}, \alpha_{k}\right)$ tal que $\mu_{G, \boldsymbol{w}}(y)=0$. 
Como $\mu_{G, \boldsymbol{w}}$ é um polinômio de emparelhamentos, então $\mu_{G, \boldsymbol{w}}$ possui coeficiente dominante positivo. Assim, $\lim _{x \rightarrow \infty} \mu_{G, \boldsymbol{w}}(x)=\infty$. Portanto, como $\mu_{G}\left(\alpha_{n-1}\right)<0$, existe $y \in \mathbb{R} \operatorname{com} y>\alpha_{n-1}$ tal que $\mu_{G, \boldsymbol{w}}(y)=0$.

Se $n$ é par, então $\lim _{x \rightarrow-\infty} \mu_{G, \boldsymbol{w}}(x)=\infty$ e $\mu_{G, \boldsymbol{w}}\left(\alpha_{1}\right)<0$. Se $n$ é ímpar, então $\lim _{x \rightarrow-\infty} \mu_{G, \boldsymbol{w}}(x)=$ $-\infty$ e $\mu_{G, \boldsymbol{w}}\left(\alpha_{1}\right)>0$. Portanto, existe $y \in \mathbb{R}$ com $y<\alpha_{1}$ tal que $\mu_{G, \boldsymbol{w}}(y)=0$.

Logo, as raízes de $\mu_{G, \boldsymbol{w}}$ são reais e $\mu_{G-v, \boldsymbol{w}}$ entrelaça estritamente $\mu_{G, \boldsymbol{w}}$.

Considere o grafo completo $K_{3}$. Note que $\mu_{K_{3}}=x^{3}-3 x$. Note também que $\mu_{K_{3}, \mathbb{1}}$ pode ser escrito da seguinte forma:

$$
\mu_{K_{3}}=x(x-\sqrt{3})(x+\sqrt{3}) .
$$

Assim, todas as raízes de $\mu_{K_{3}}$ são reais.

Para provar que os polinômios característicos de todos os grafos com pesos não negativos nas arestas possuem somente raízes reais, utilizamos o argumento de continuidade de raízes do Corolário 4.15. Para satisfazer as condições desse corolário, mostraremos que os polinômios de emparelhamentos de grafos, cujos pesos nas arestas convergem para $w$, convergem uniformemente para o polinômio de emparelhamentos do grafo com peso $w$ nas arestas.

Teorema 6.11. Sejam $X \subseteq \mathbb{C}$ compacto e $G=\left(V,\left(\begin{array}{c}V \\ 2\end{array}\right)\right)$ um grafo e seja $w_{0}:\left(\begin{array}{c}V \\ 2\end{array}\right) \rightarrow \mathbb{R}_{+}$. Tome $E:=\left\{e \in\left(\begin{array}{c}V \\ 2\end{array}\right): w_{0}(e)=0\right\}$ e $w_{k}:=w_{0}+\frac{1}{k} \mathbb{1}_{E}$ para todo $k \in \mathbb{N} \backslash\{0\}$. Então $\left(\mu_{G, w_{k}}\right)_{k \in \mathbb{N} \backslash\{0\}}$ converge uniformemente para $\mu_{G, \boldsymbol{w}_{0}}$ em $X$.

Demonstração. Note que

$$
\mu_{G, \boldsymbol{w}_{n}}(x)=\sum_{d \in \mathbb{N}} x^{|V|-2 d}(-1)^{d} \mathrm{~m}_{G, \boldsymbol{w}_{n}}(d)
$$

para todo $n \in \mathbb{N}$. Além disso, vale que

$$
\lim _{n \rightarrow \infty}(-1)^{d} \mathrm{~m}_{G, \boldsymbol{w}_{\boldsymbol{k}}}(d)=(-1)^{d} \mathrm{~m}_{G, \boldsymbol{w}_{\mathbf{0}}}(d)
$$

para todo $d \in \mathbb{N}$. Logo, o resultado segue do Teorema 4.16 .

Utilizamos o resultado acima e o Corolário 4.15, que é uma implicação do resultado de Hurwitz, para mostrar que as raízes dos polinômios de emparelhamentos cujos pesos nas arestas convergem para $w$, que são reais, convergem para as raízes do polinômio de emparelhamentos com peso $w$ nas arestas e, portanto, essas raízes também são reais.

Corolário 6.12. Seja $G=\left(V,\left(\begin{array}{l}V \\ 2\end{array}\right)\right)$ um grafo com pesos nas arestas e $w:\left(\begin{array}{l}V \\ 2\end{array}\right) \rightarrow \mathbb{R}_{+}$. Então o polinômio $\mu_{G, \boldsymbol{w}}$ possui apenas raízes reais.

Demonstração. Defina $\Omega:=\mathbb{C} \backslash \mathbb{R}$. Note que $\Omega$ é aberto e conexo. Seja $E:=\left\{e \in\left(\begin{array}{c}V \\ 2\end{array}\right): w(e)=0\right\}$. Defina $w_{k}:=w+\frac{1}{k} \mathbb{1}_{E}$ para todo $k \in \mathbb{N} \backslash\{0\}$.

Pelo Teorema 6.10 , temos que $\mu_{G, \boldsymbol{w}_{\boldsymbol{k}}}(x) \neq 0$ para todo $k \in \mathbb{N} \backslash\{0\}$ e todo $x \in \Omega$. Além disso, pelo Teorema 6.11 , temos que $\left(\mu_{G, \boldsymbol{w}_{\boldsymbol{k}}}\right)_{k \in \mathbb{N} \backslash\{0\}}$ converge uniformemente para $\mu_{G, \boldsymbol{w}}$ em todo subconjunto compacto de $\Omega$. Portanto, pelo Corolário 4.15, temos que $\mu_{G, \boldsymbol{w}}(x) \neq 0$ para todo $x \in \Omega$.

Considere o grafo completo $K_{3}=(V, E)$. Sejam $G_{1}=\left(V, E_{1}\right)$ e $G_{2}=\left(V, E_{2}\right)$ subgrafos de $K_{3}$ com $\left|E_{1}\right|=1$ e $\left|E_{2}\right|=2$. Note que $\mu_{G_{1}}(x)=x^{3}-x$ e $\mu_{G_{2}}=x^{3}-2 x$. Note também que $\mu_{G_{1}}$ e $\mu_{G_{2}}$ podem ser escritos da seguinte forma:

$$
\mu_{G_{1}}(x)=x(x-1)(x+1) \quad \text { e } \quad \mu_{G_{1}}(x)=x(x-\sqrt{2})(x+\sqrt{2}) .
$$


Assim, os polinômios de emparelhamentos de $G_{1}$ e $G_{2}$ possuem somente raízes reais. Como todos os subgrafos não-simples de $K_{3}$ são isomorfos a $G_{1}$ e $G_{2}$, então os polinômios de emparelhamentos de todos os subgrafos de $K_{3}$ possuem somente raízes reais.

Além de possuírem somente raízes reais, as raízes dos polinômios de emparelhamentos de um grafo são limitadas superiormente por um valor que depende do grau máximo desse grafo. Além disso, existe um outro limitante para as raízes dos polinômios de emparelhamentos de grafos conexos $G$, que é o raio espectral da cobertura universal de $G$.

\section{Grau máximo}

Mostraremos que todas as raízes do polinômio de emparelhamentos de $G$ são limitadas superiormente por $2 \sqrt{\Delta(G)-1}$. Assim, se $G$ é um grafo $d$-regular, então as raízes do polinômio de emparelhamentos de $G$ são no máximo $2 \sqrt{d-1}$.

Teorema 6.13 ([16, Lema 4.4]). Seja $G=(V, E)$ um grafo conexo com $\Delta(G)>1$. Seja $\varnothing \neq S \subsetneq V$. Defina $\alpha:=\sqrt{\Delta(G)-1}$. Então, para todo $v \in V \backslash S$ tal que $\Gamma_{G}(v) \cap S \neq \varnothing$ vale que

$$
\frac{\mu_{G-S}(x)}{\mu_{G-S-v}(x)} \geq \alpha \quad \text { e } \quad \mu_{G-S}(x)>0
$$

para todo número real $x \geq 2 \alpha$.

Demonstração. Defina $n:=|V|$.

Provaremos esse resultado por indução em $n-|S|$. Se $n-|S|=1$, então $\mu_{G-S}(x)=x$ e $\mu_{G-S-v}(x)=1$ para todo $v \in V \backslash S$. Logo, como $\alpha \geq 1$, então

$$
\frac{\mu_{G-S}(x)}{\mu_{G-S-v}(x)}=x \geq 2 \alpha \geq \alpha \quad \text { e } \quad \mu_{G-S}(x)>0
$$

para todo número real $x \geq 2 \alpha$.

Suponha que $n-|S|>1$. Pela hipótese de indução, temos que

$$
\frac{\mu_{G-S-v}(x)}{\mu_{G-S-v-u}(x)} \geq \alpha \quad \text { e } \quad \mu_{G-S-v}(x)>0
$$

para todos $v \in V \backslash S, u \in \Gamma_{G-S}(v)$ e todo número real $x \geq 2 \alpha$.

Logo, pelo Teorema 2.61, temos que

$$
\frac{\mu_{G-S}(x)}{\mu_{G-S-v}(x)}=x-\frac{\sum_{u \in \Gamma_{G-S}(v)} \mu_{G-S-v-u}(x)}{\mu_{G-S-v}(x)} \geq x-\sum_{u \in \Gamma_{G-S}(v)} \frac{1}{\alpha}
$$

para todo $v \in V \backslash S$ e todo número real $x \geq 2 \alpha$. Note que $\operatorname{grau}_{G-S}(v) \leq \Delta(G)-1$ para todo $v \in V \backslash S$ com $\Gamma_{G}(v) \cap S \neq \varnothing$. Portanto, vale que

$$
\frac{\mu_{G-S}(x)}{\mu_{G-S-v}(x)} \geq x-\frac{\Delta(G)-1}{\alpha}=x-\frac{\alpha^{2}}{\alpha} \geq 2 \alpha-\alpha=\alpha
$$

para todo $v \in V \backslash S \operatorname{com} \Gamma_{G}(v) \cap S \neq \varnothing$ e todo número real $x \geq 2 \alpha$.

Como $G$ é conexo, existe $v \in V \backslash S$ tal que $\Gamma_{G}(v) \cap S \neq \varnothing$. Assim, por (6.1) e (6.2), temos que

$$
\mu_{G-S}(x)>0
$$

para todo número real $x \geq 2 \alpha$. 
Teorema 6.14 ([16, Teorema 4.3]). Seja $G=(V, E)$ um grafo conexo com $n$ vértices tal que $\Delta(G)>1$. Então $r_{i}\left(\mu_{G}\right) \leq 2 \sqrt{\Delta(G)-1}$ para todo $i \in[n]$.

Demonstração. Seja $v \in V$. Defina $\alpha:=\sqrt{\Delta(G)-1}$. Pelo Teorema 6.13, temos que

$$
\frac{\mu_{G-v}(x)}{\mu_{G-v-u}(x)} \geq \alpha \quad \text { e } \quad \mu_{G-v}(x)>0
$$

para todo $u \in \Gamma_{G}(v)$ e todo número real $x \geq 2 \alpha$. Logo, pelo Teorema 2.61, temos que

$$
\begin{aligned}
\frac{\mu_{G}(x)}{\mu_{G-v}(x)} & =x-\frac{\sum_{u \in \Gamma_{G}(v)} \mu_{G-v-u}(x)}{\mu_{G-v}(x)} & \\
& \geq x-\sum_{u \in \Gamma_{G}(v)} \frac{1}{\alpha} \geq x-\frac{\Delta(G)}{\alpha} & \forall x \geq 2 \alpha .
\end{aligned}
$$

Seja $x>2 \alpha$ real. Note que

$$
x-\frac{\Delta(G)}{\alpha}>2 \alpha-\frac{\Delta(G)}{\alpha}=\alpha\left(2-\frac{\Delta(G)}{\alpha^{2}}\right)=\alpha\left(2-\frac{\Delta(G)}{\Delta(G)-1}\right) .
$$

Como $\Delta(G) \geq 2$, então $\alpha\left(2-\frac{\Delta(G)}{\Delta(G)-1}\right) \geq 0$, o que implica que $\frac{\mu_{G}(x)}{\mu_{G-v}(x)}>0$ para todo número real $x>2 \alpha$. Portanto, como $\mu_{G-v}(x)>0$, então $\mu_{G}(x)>0$ para todo número real $x>2 \alpha$.

\section{Raio espectral da cobertura universal}

Mostraremos que as raízes dos polinômios de emparelhamentos de um grafo conexo $G$ são limitadas superiormente pelo raio espectral da cobertura universal de $G$. Esse resultado é obtido limitando as raízes do polinômio de emparelhamentos de $G$ pelo raio espectral da árvore de caminhos de $G$, definida nas preliminares.

Teorema 6.15 (vide [11]). Seja $G=(V, E)$ um grafo e $v \in V$. Então

$$
\frac{\mu_{G}}{\mu_{G-v}}=\frac{\mu_{P(G, v)}}{\mu_{P(G, v)-v}}
$$

e $\mu_{G}$ divide $\mu_{P(G, v)}$.

Demonstração. Defina $n:=|V|$. Provaremos esse resultado por indução em $n$.

Note que se $G$ é uma árvore, então $G$ é isomorfo a $P(G, v)$. Logo, se $n=1$, então $G$ é uma árvore, o que implica que $\mu_{G}=\mu_{P(G, v)}$. Assim, como $\mu_{G-v}=\mu_{P(G, v)-v}=1$, então

$$
\frac{\mu_{G}}{\mu_{G-v}}=\frac{\mu_{P(G, v)}}{\mu_{P(G, v)-v}} .
$$

Além disso, como $\mu_{G}=\mu_{P(G, v)}$, então $\mu_{G}$ divide $\mu_{P(G, v)}$.

Suponha que $n \geq 2$. Pelo Teorema 2.61, temos que

$$
\frac{\mu_{G}}{\mu_{G-v}}=\frac{x \mu_{G-v}-\sum_{u \in \Gamma_{G}(v)} \mu_{G-v-u}}{\mu_{G-v}}=x-\frac{\sum_{u \in \Gamma_{G}(v)} \mu_{G-v-u}}{\mu_{G-v}} .
$$


Pela hipótese de indução, temos que

$$
\begin{aligned}
\frac{\sum_{u \in \Gamma_{G}(v)} \mu_{G-v-u}}{\mu_{G-v}} & =\sum_{u \in \Gamma_{G}(v)} \frac{\mu_{P(G-v, u)-u}}{\mu_{P(G-v, u)}} \\
& =\frac{\sum_{u \in \Gamma_{G}(v)}\left(\mu_{P(G-v, u)-u} \prod_{w \in \Gamma_{G}(v) \backslash\{u\}} \mu_{P(G-v, w)}\right)}{\prod_{u \in \Gamma_{G}(v)} \mu_{P(G-v, u)}} \\
& =\frac{\sum_{u \in \Gamma_{G}(v)} \mu_{P(G, u)-v-u}}{\mu_{P(G, v)-v}}
\end{aligned}
$$

Logo, por (6.3) e pelo Teorema 2.61, temos que

$$
\frac{\mu_{G}}{\mu_{G-v}}=\frac{x \mu_{P(G, v)-v}-\sum_{u \in \Gamma_{G}(v)} \mu_{P(G, u)-v-u}}{\mu_{P(G, v)-v}}=\frac{\mu_{P(G, v)}}{\mu_{P(G, v)-v}} .
$$

Pela hipótese de indução, temos que $\mu_{G-v}$ divide $\mu_{P(G, v)-v}$, o que implica que $\mu_{G}$ divide $\mu_{P(G, v)}$.

Corolário 6.16 ([11, Teorema 2.5]). Seja $G=(V, E)$ um grafo e $v \in V$. Então $x \leq \rho(P(G, v))$ para todo $x \in \mathbb{R} \operatorname{com} \mu_{G}(x)=0$.

Demonstração. Defina $p$ como o polinômio característico da matriz de adjacência de $P(G, v)$. Pelo Teorema 6.15, temos que $\mu_{G}$ divide $\mu_{P(G, v)}$. Como $P(G, v)$ é uma árvore, então, pelo Teorema 2.62, temos que $\mu_{P(G, v)}=p$, o que implica que $\mu_{G}$ também divide $p$. Logo, vale que $p(x)=0$ para todo $x \in \mathbb{R} \operatorname{com} \mu_{G}(x)=0$. Portanto

$$
x \leq r_{\max }(p) \leq \rho(P(G, v))
$$

para todo $x \in \mathbb{R} \operatorname{com} \mu_{G}(x)=0$.

Teorema 6.17 ([24, Lema 3.5]). Seja $G$ um grafo. Então $x \leq \rho\left(T_{G}\right)$ para todo $x \in \mathbb{R}$ tal que $\mu_{G}(x)=0$.

Demonstração. Seja $v \in V(G)$. Defina $V_{P}:=V(P(G, v))$. Defina também $A_{T}$ e $A_{P}$ como as matrizes de adjacência de $T_{G}$ e $P(G, v)$, respectivamente. Note que, para todo caminho $p$ em $G$, vale que $C_{G, p} \subseteq P_{G, p}$. Assim, como $T_{G}$ e $P(G, v)$ são árvores, então $P(G, v)=T_{G}\left[V_{P}\right]$. Logo, vale que

$$
A_{P}=A_{T}\left[V_{P}\right]
$$

Pelo Corolário 6.16, para todo $x \in \mathbb{R} \operatorname{com} \mu_{G}(x)=0$, vale que $x \leq \rho(P(G, v))$. Note que

$$
\begin{aligned}
\rho(P(G, v)) & =\left\|A_{P}\right\|_{2} \\
& =\max \left\{\left\|A_{P} x\right\|_{2}: x \in \mathbb{R}^{V_{P}} \text { e }\|x\|_{2}=1\right\} \\
& \leq \sup \left\{\left\|A_{T} x\right\|_{2}: x \in \mathbb{R}^{V_{T}} \text { e }\|x\|_{2}=1\right\} \\
& =\left\|A_{T}\right\|_{2}=\rho\left(T_{G}\right) .
\end{aligned}
$$

Portanto, vale que $x \leq \rho\left(T_{G}\right)$ para todo $x \in \mathbb{R} \operatorname{com} \mu_{G}(x)=0$. 


\subsection{Construção de grafos expansores}

Nesta seção, combinamos as ferramentas apresentadas anteriormente para provar a existência de famílias infinitas de grafos de Ramanujan.

Utilizamos o Teorema 6.9 para mostrar que a soma dos polinômios característicos das matrizes de sinais dos 2-lifts de um grafo $G$ possui as mesmas raízes que o polinômio de emparelhamentos de G. Pelo Teorema 5.30, os polinômios característicos das matrizes de sinais dos 2-lifts de um grafo $G$ formam uma árvore entrelaçadora de polinômios. Assim, podemos utilizar a propriedade de raízes máximas apresentada no Corolário 4.12: as raízes do polinômio característico da matriz de sinais de pelo menos um dos 2-lifts de $G$ são limitadas superiormente pela raiz máxima da soma dos polinômios característicos das matrizes de sinais de todos os 2-lifts de $G$, que é igual à raiz máxima do polinômio de emparelhamentos de $G$. Além disso, se considerarmos que $G$ é $d$-regular e conexo, então as raízes dos polinômios de emparelhamentos de $G$ são reais e limitadas superiormente por $2 \sqrt{d-1}$, como mostrado no Corolário 6.12 e no Teorema 6.14. Dessa forma, as raízes do polinômio característico da matriz de sinais de pelo menos um dos 2-lifts de $G$ são limitadas superiormente por $2 \sqrt{d-1}$. Como mostrado no Teorema 6.8, os autovalores da matriz de adjacência dos 2-lifts de $G$ são a união dos autovalores de $A_{G}$ com os autovalores da matriz de sinais do 2-lift em questão, levando em consideração a multiplicidade dos autovalores. Assim, pelo menos um 2-lift de um grafo de Ramanujan é conexo e possui matriz de adjacência cujos autovalores não-triviais são limitados superiormente por $2 \sqrt{d-1}$. Além disso, como 2-lifts de grafos regulares também são regulares de mesma valência, então os 2-lifts de $G$ também são $d$-regulares, como mostrado na Proposição 6.2.

Teorema 6.18 (Marcus, Spielman e Srivastava [24]). Seja $G$ um grafo com $n$ vértices. Então existe um 2-lift $H$ de $G$ tal que

$$
\lambda^{\downarrow}\left(A_{G, s}\right) \leq r_{\max }\left(\mu_{G}(x)\right) \mathbb{1},
$$

onde $s$ é a função de sinais do 2-lift $H$ de $G$.

Demonstração. Tome $E:=E(G)$ e $n:=|V(G)|$. Defina $f_{G, s}(x):=\operatorname{det}\left(x I-A_{G, s}\right)$ para todo $s \in\{ \pm 1\}^{E}$. Pelo Teorema 6.9, temos que

$$
\frac{1}{\left|\{ \pm 1\}^{E}\right|} \sum_{s \in\{ \pm 1\}^{E}} f_{G, s}(x)=\mu_{G}(x) .
$$

Logo, pelo Corolário 4.12 e pelo Teorema 5.30, existe $\hat{s} \in\{ \pm 1\}^{E}$ tal que

$$
\begin{aligned}
r_{\max }\left(f_{G, \hat{s}}(x)\right) & \leq r_{\max }\left(\sum_{s \in\{ \pm 1\}^{E}} f_{G, s}(x)\right) \\
& =r_{\max }\left(\left|\{ \pm 1\}^{E}\right| \mu_{G}(x)\right)=r_{\max }\left(\mu_{G}(x)\right) .
\end{aligned}
$$

Como $\hat{s} \in\{ \pm 1\}^{E}$, então existe um 2-lift $\hat{H}$ de $G$ cuja função de sinais é $\hat{s}$. Portanto, como $r_{\max }\left(f_{G, \hat{s}}\right) \leq r_{\max }\left(\mu_{G}(x)\right)$, então

$$
\lambda_{i}^{\downarrow}\left(A_{G, \hat{s}}\right) \leq r_{\max }\left(\mu_{G}(x)\right)
$$

para todo $i \in[n]$. 
Corolário 6.19 (Marcus, Spielman e Srivastava [24]). Seja $G$ um grafo conexo e $d$-regular com $d>2$. Então existe um 2-lift $H$ de $G$ tal que

$$
\lambda^{\downarrow}\left(A_{G, s}\right) \leq(2 \sqrt{d-1}) \mathbb{1},
$$

onde $s$ é a função de sinais do 2-lift $H$ de $G$.

Demonstração. Seja $n:=|V(G)|$. Pelo Corolário 6.12 e pelo Teorema 6.14, temos que $r_{\max }\left(\mu_{G}(x)\right) \leq$ $2 \sqrt{d-1}$. Logo, pelo Teorema 6.18, existe um 2-lift $H$ de $G$ tal que

$$
\lambda_{i}^{\downarrow}\left(A_{G, s}\right) \leq r_{\max }\left(\mu_{G}(x)\right) \leq 2 \sqrt{d-1}
$$

para todo $i \in[n]$, onde $s$ é a função de sinais do 2-lift $H$ de $G$.

Note que, dessa forma, podemos construir iterativamente, a partir de um grafo de Ramanujan, uma família infinita de grafos $d$-regulares cujos autovalores não-triviais de suas matrizes de adjacência são limitados superiormente por $2 \sqrt{d-1}$, que é uma família expansora ótima.

Como os autovalores não-triviais das matrizes de adjacência dos grafos dessas famílias podem não ser limitados inferiormente por $-2 \sqrt{d-1}$, então essas famílias expansoras ótimas obtidas não são necessariamente famílias de grafos de Ramanujan. Para construir especificamente famílias de grafos de Ramanujan, adicionamos uma restrição de que os grafos dessas famílias devem ser construídos a partir de grafos de Ramanujan bipartidos. Assim, como todos os 2-lifts de um grafo bipartido também são bipartidos (Proposição 6.3), então todos os grafos dessa família são bipartidos. Dessa forma, os valores absolutos de todos os autovalores não-triviais das matrizes de adjacência dos grafos dessas famílias serão limitados superiormente por $2 \sqrt{d-1}$. Assim, essas famílias construídas iterativamente a partir de grafos de Ramanujan bipartidos são famílias infinitas de grafos de Ramanujan de mesma valência, como mostrado a seguir.

Corolário 6.20. Seja $G$ um grafo de Ramanujan $d$-regular bipartido. Então existe um 2-lift $H$ de $G$ tal que $H$ é um grafo de Ramanujan $d$-regular bipartido.

Demonstração. Pelo Corolário 6.19, temos que existe um 2-lift $H$ de $G$ tal que $\lambda^{\downarrow}\left(A_{G, s}\right) \leq(2 \sqrt{d-1}) \mathbb{1}$, onde $s$ é a função de sinais do 2-lift $H$ de $G$.

Seja $n:=|V(G)|$. Como $G$ é um grafo de Ramanujan bipartido, então $\left|\lambda_{i}^{\downarrow}\left(A_{G}\right)\right| \leq 2 \sqrt{d-1}$ para todo $i \in\{2, \ldots, n-1\}$. Assim, pelo Teorema 6.8 , temos que $\lambda_{i}^{\downarrow}\left(A_{H}\right) \leq 2 \sqrt{d-1}$ para todo $i \in\{2, \ldots, 2 n-1\}$.

Pelas Propriedades 6.2 e 6.3, temos que $H$ é $d$-regular e bipartido. Assim, pelo Teorema 2.50, temos que $\left|\lambda_{i}^{\downarrow}\left(A_{H}\right)\right| \leq 2 \sqrt{d-1}$ para todo $i \in\{2, \ldots, 2 n-1\}$ e, como $H$ é $d$-regular, então $H$ é um grafo de Ramanujan.

Grafos bipartidos completos $K_{n, n}$ são exemplos de grafos de Ramanujan bipartidos (vide Proposição 2.55). Logo, podemos construir famílias infinitas de grafos de Ramanujan a partir de um grafo bipartido completo.

Para uma noção mais intuitiva, segue um algoritmo que constrói famílias de grafos de Ramanujan, onde a função 2-lifts $(G)$ retorna o conjunto de 2-lifts, não isomorfos entre si, do grafo $G$. 


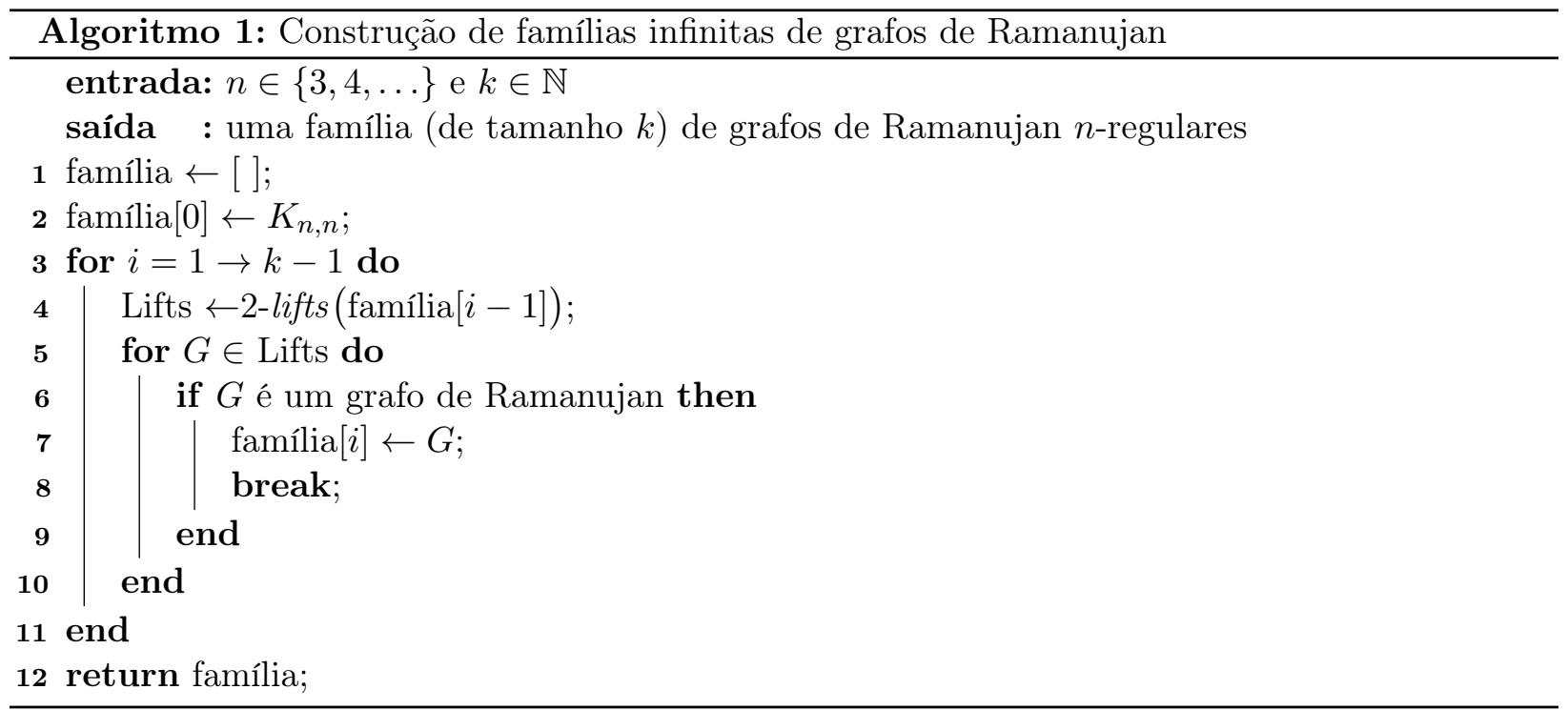

Apresentamos o algoritmo acima apenas para mostrar a ideia da prova de existência de famílias infinitas de grafos de Ramanujan. Construir essas famílias dessa forma é infactível devido ao tempo de execução desse algoritmo.

\subsection{Construção de grafos expansores irregulares}

A prova de existência de famílias expansoras irregulares ótimas é semelhante à prova de existência de famílias expansoras regulares ótimas mostrada anteriormente. Pelo Teorema 6.18, temos que os autovalores da matriz de sinais de pelo menos um dos 2-lifts de um grafo $G$ são limitados superiormente pela raiz máxima do polinômio de emparelhamentos de $G$. Como, pelo Teorema 6.17, o polinômio de emparelhamentos de um grafo $G$ possui apenas raízes reais e limitadas superiormente por $\rho\left(T_{G}\right)$, então todos os autovalores da matriz de sinais de pelo menos um dos 2-lifts de um grafo $G$ são limitados superiormente por $\rho\left(T_{G}\right)$.

Corolário 6.21 (Marcus, Spielman e Srivastava [24]). Seja $G$ um grafo. Então existe um 2-lift $H$ de $G$ tal que $\lambda^{\downarrow}\left(A_{G, s}\right) \leq \rho\left(T_{G}\right) \mathbb{1}$, onde $s$ é a função de sinais do 2-lift $H$ de $G$.

Demonstração. Pelo Corolário 6.12 e pelo Teorema 6.17, temos que $r_{\max }\left(\mu_{G}(x)\right) \leq \rho\left(T_{G}\right)$. Logo, pelo Teorema 6.18, existe um 2-lift $H$ de $G$ tal que

$$
\lambda^{\downarrow}\left(A_{G, s}\right) \leq r_{\max }\left(\mu_{G}(x)\right) \mathbb{1} \leq \rho\left(T_{G}\right) \mathbb{1},
$$

onde $s$ é a função de sinais do 2-lift $H$ de $G$.

Pelas Proposições 6.4 e 6.6, temos que, se um grafo $G$ é birregular, então todos os 2-lifts desse grafo são birregulares e possuem coberturas universais isomorfas a $T_{G}$. Dessa forma, os autovalores da matriz de sinais de pelo menos um dos 2-lifts $H$ de $G$ são limitados superiormente por $\rho\left(T_{H}\right)=\rho\left(T_{G}\right)$. Seja $G$ um grafo birregular cujos autovalores não-triviais de $A_{G}$ são limitados superiormente por $\rho\left(T_{G}\right)$. Como os autovalores da matriz de adjacência dos 2-lifts de $G$ são a união entre os autovalores de $G$ e os autovalores da matriz de sinais do 2-lift em questão (Teorema 6.8), então todos os autovalores não triviais da matriz de adjacência de pelo menos um dos 2-lifts de $G$ 
são limitados superiormente por $\rho\left(T_{G}\right)$. Logo, podemos construir famílias expansoras irregulares ótimas a partir de um grafo birregular cujos autovalores não-triviais da sua matriz de adjacência são limitados superiormente pelo raio espectral da sua cobertura universal.

Note que o algoritmo que constrói famílias expansoras irregulares ótimas é semelhante ao Algoritmo 1. 


\section{Capítulo 7}

\section{Construção de expansores por esparsificadores espectrais}

No capítulo anterior, mostramos a existência de grafos expansores regulares e irregulares utilizando construções iterativas de 2-lifts. Neste capítulo, mostraremos a existência de bons esparsificadores espectrais de grafos completos, que podem ser considerados expansores irregulares com pesos nas arestas. A existência de tais grafos foi provada por Batson, Spielman e Srivastava [2] através da prova de existência de bons esparsificadores espectrais de grafos arbitrários que possuem no máximo o dobro do número de arestas de um grafo de Ramanujan $d$-regular de mesmo tamanho. Quando mencionamos bons esparsificadores espectrais, nos referimos a aproximações pelo menos tão boas quanto aproximações de grafos completos por grafos de Ramanujan não-bipartidos.

Esparsificadores são subgrafos esparsos de um grafo que se comportam de forma semelhante ao grafo original em determinadas características. Por exemplo, esparsificadores de corte [3] de um grafo são subgrafos cujo peso de cada corte é próximo do peso do respectivo corte do grafo original. Outro exemplo de esparsificadores são os esparsificadores espectrais, que são subgrafos cuja forma quadrática de seu Laplaciano é próxima da forma quadrática do Laplaciano do grafo original. Seja $G=(V, E, w)$ um grafo com pesos nas arestas. Denote $w_{G}(S):=\sum_{e \in \delta_{G}}(S) w_{e}$. Note que, para todo subconjunto de vértices $S \subseteq V$, vale que $\mathbb{1}_{S} L_{G} \mathbb{1}_{S}=w_{G}(S)$. Assim, a soma dos pesos das arestas em qualquer corte de um esparsificador espectral é uma boa aproximação da soma dos pesos das arestas no respectivo corte do grafo original. Dessa forma, podemos considerar que esparsificadores espectrais são esparsificadores de corte.

Definição 7.1. Seja $\varepsilon>0$. Dizemos que um grafo com pesos nas arestas $H=(V, F, \bar{w})$ é uma $(1+\varepsilon)$-aproximação de um grafo com pesos nas arestas $G=(V, E, w)$ se $F \subseteq E$ e se

$$
L_{G} \preceq L_{H} \preceq(1+\varepsilon) L_{G} .
$$

Um grafo $H$ com pesos nas arestas é um esparsificador espectral de um grafo $G$ com pesos nas arestas se $H$ é um subgrafo esparso de $G$ e se existe $\varepsilon>0$ próximo de 0 tal que $H$ seja uma $(1+\varepsilon)$-aproximação de $G$. Assim, se $H$ é um esparsificador espectral de um grafo $G=(V, E)$ tal que $H$ é uma $(1+\varepsilon)$-aproximação de $G$, então $w_{G}(S) \leq w_{H}(S) \leq(1+\varepsilon) w_{G}(S)$ para todo $S \subseteq V$. Dessa forma, a soma dos pesos das arestas em qualquer corte $\delta_{H}(S)$ é uma boa aproximação da soma dos pesos das arestas de $\delta_{G}(S)$.

Batson, Spielman e Srivastava provaram a existência de esparsificadores espectrais que são $(1+\varepsilon)$-aproximações de grafos arbitrários com um número de arestas linear (em relação ao número de vértices), onde $\varepsilon$ é próximo de 0 . Esse resultado foi obtido a partir de um algoritmo, o 
que nos permite obter bons esparsificadores espectrais, e mais especificamente, grafos expansores a partir da esparsificação de grafos completos. Mais adiante, apresentaremos a ideia de construir esses esparsificadores espectrais, aresta por aresta, com o auxílio de limitantes de autovalores, que chamamos de barreiras.

Construímos um esparsificador espectral de um grafo completo $K_{n}=(V, E)$ de forma iterativa. A cada iteração $i$ obtemos um grafo $H_{i}=\left(V, E, w_{i}\right)$, com $w_{0}=0$, de forma que os autovalores não nulos do Laplaciano de $H_{i}$ sejam limitados por $\ell_{i}$ e $u_{i}$, que são as barreiras inferior e superior associadas a $i$-ésima iteração. Essas barreiras são aumentadas de uma constante a cada iteração, de modo que, a partir de uma certa iteração, a razão entre a barreira superior e a barreira inferior seja pequena (próxima de 1). De acordo com o resultado de Batson, Spielman e Srivastava, para cada iteração $i$, existem uma aresta $f \in E$ e um número real positivo $\beta$ tais que os autovalores não nulos do Laplaciano do grafo

$$
H_{i}:=\left(V, E, w_{i-1}+\beta e_{f}\right)
$$

são limitados inferior e superiormente por $\ell_{i}$ e $u_{i}$, respectivamente. Dessa forma, em todas as iterações, os autovalores não nulos do Laplaciano de $H_{i}$ estão entre as barreiras $\ell_{i}$ e $u_{i}$. Como a razão entre as barreiras inferior e superior é próxima de 1 a partir de um certo número de iterações, então, na $k$-ésima iteração, onde $k$ é um número natural suficientemente grande porém linear em $n$, os autovalores não-nulos do Laplaciano do grafo $\left(V, E, w_{k} / \ell_{k}\right)$ estão no intervalo $[1,1+\varepsilon]$, com $\varepsilon>0$ próximo de 0. Dessa forma, como $\lambda_{1}^{\downarrow}\left(L_{K_{n}}\right)=\lambda_{2}^{\downarrow}\left(L_{K_{n}}\right)=\cdots=\lambda_{n-1}^{\downarrow}\left(L_{K_{n}}\right)=n$, então o grafo $\left(V, E, \gamma w_{k}\right)$ é um bom esparsificador espectral de $K_{n}$, onde $\gamma=\frac{n}{\ell_{k}}$.

A construção de esparsificadores espectrais de grafos arbitrários, que será apresentada mais adiante, é semelhante à construção apresentada anteriormente para grafos completos. Porém, a intuição da construção de esparsificadores de grafos completos é mais simples devido à uniformidade de alguns de seus autovalores.

Além disso, é possível mostrar que bons esparsificadores espectrais de grafos completos também satisfazem as propriedades principais de grafos expansores mostradas no Capítulo 3. Assim, podemos considerar bons esparsificadores espectrais de grafos completos como bons expansores, apesar de serem irregulares e possuírem pesos nas arestas.

\subsection{Funções potenciais e barreiras}

Para construir esparsificadores espectrais de grafos arbitrários, utilizamos uma técnica de controle dos autovalores de matrizes por barreiras. As barreiras são representadas por funções monótonas $\ell: \mathbb{N} \rightarrow \mathbb{R}$ e $u: \mathbb{N} \rightarrow \mathbb{R}$, que são os valores das barreiras inferior e superior a cada iteração. Sejam $\ell_{0}$ e $u_{0}$ os valores iniciais das barreiras e $\delta_{\ell}$ e $\delta^{u}$ as constantes que são somadas às barreiras a cada iteração. Os valores das barreiras inferior e superior na $i$-ésima iteração são $\ell_{i}=\ell_{0}+i \delta_{\ell}$ e $u_{i}=u_{0}+i \delta^{u}$, respectivamente. Elas são utilizadas para limitar inferior e superiormente os autovalores não nulos do Laplaciano do esparsificador em progresso em cada iteração. Para garantir que as barreiras sejam de fato limitantes dos autovalores, utilizamos funções potenciais de matrizes (associadas a cada uma das barreiras), que quantificam a proximidade dos autovalores de uma matriz em relação às barreiras. As funções potenciais são escolhidas para que, à medida que pelo menos um dos autovalores de uma matriz $A$ se aproxima de uma das barreiras, as funções potenciais de $A$ associadas à barreira em questão tendem para infinito.

Definição 7.2. Sejam $\ell, u \in \mathbb{R}$. Defina as funções potenciais inferior e superior associadas a $\ell$ e $u$, 

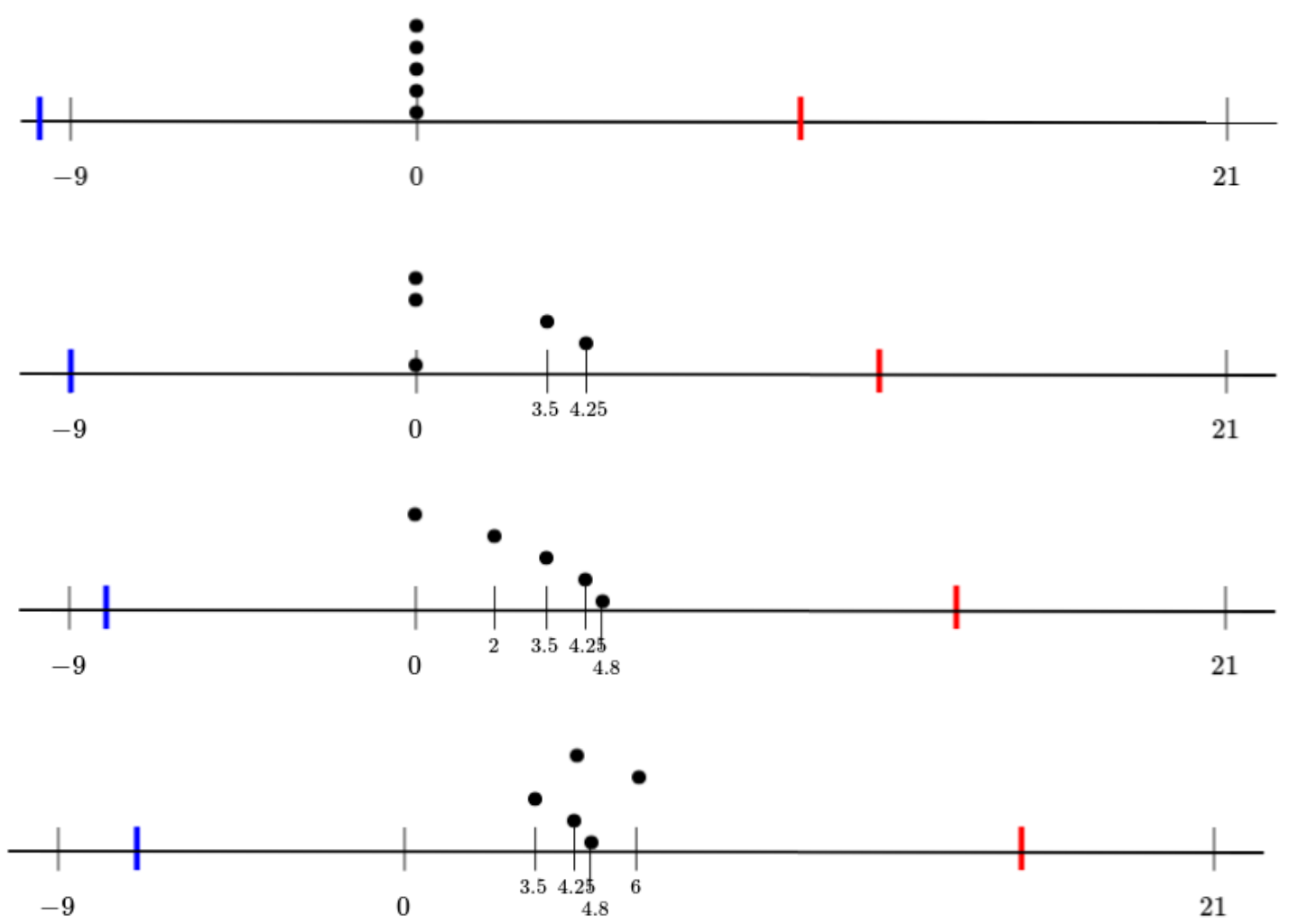

Figura 7.1: Representação das barreiras $\ell$ (em azul) e $u$ (em vermelho) e dos autovalores não nulos do Laplaciano do esparsificador em progresso em cada uma das iterações. 
respectivamente:

$$
\begin{aligned}
\Phi_{\ell}(A) & :=\operatorname{Tr}\left((A-\ell I)^{-1}\right)=\sum_{i \in[n]} \frac{1}{\lambda_{i}^{\downarrow}(A)-\ell}=\sum_{i \in[n]} \lambda_{i}^{\downarrow}(A-\ell I)^{-1}, \\
\Phi^{u}(A) & :=\operatorname{Tr}\left((u I-A)^{-1}\right)=\sum_{i \in[n]} \frac{1}{u-\lambda_{i}^{\downarrow}(A)}=\sum_{i \in[n]} \lambda_{i}^{\downarrow}(u I-A)^{-1}
\end{aligned}
$$

para toda matriz simétrica $A \in \mathbb{R}^{n \times n}$ com $\ell I \prec A \prec u I$.

Note que o potencial de uma matriz associado a uma barreira é a soma de termos que quantificam a proximidade entre cada um dos autovalores da matriz e a barreira em questão. Dessa forma, os autovalores que estão afastados da barreira contribuem menos para a função potencial do que os autovalores mais próximos a ela. Assim, à medida que pelo menos um dos autovalores se aproxima da barreira, a função potencial tende para infinito.

A construção de esparsificadores espectrais é baseada em sucessivas atualizações de posto 1 de uma matriz que é inicialmente 0 e, a partir dessa matriz, pode-se obter o Laplaciano do esparsificador espectral por um ajuste de coordenadas. Por esse motivo, ao limitar os autovalores da matriz formada por atualizações de posto $1 \mathrm{em}$ cada iteração, estamos limitando também os autovalores dos esparsificadores espectrais em progresso. Mostraremos que, se os potenciais dessas matrizes não aumentam à medida que as barreiras se movem, então seus autovalores permanecem entre as barreiras. Dessa forma, é possível limitar inferior e superiormente os autovalores do Laplaciano dos esparsificadores espectrais em progresso utilizando matrizes formadas por atualizações de posto 1 cujas funções potenciais não aumentam a cada iteração.

Suponha que $A$ é uma matriz simétrica e $A+\alpha v v^{\top}$ é a atualização de posto 1 de $A$ em determinada iteração. Sejam $\ell$ e $u$ barreiras que limitam inferior e superiormente os autovalores de $A$, respectivamente. Mostraremos no Lema 7.4 que, se a barreira atualizada $\ell+\delta_{\ell}$ limita inferiormente os autovalores de $A$ e, além disso, se $\alpha$ é limitado inferiormente por $\left(v^{\top} L_{A}\left(\ell, \delta_{\ell}\right) v\right)^{-1}$ (onde $L_{A}\left(\ell, \delta_{\ell}\right)$ é uma matriz que será definida mais adiante e que depende de $A, \ell$ e $\delta_{\ell}$ ), então vale que $\Phi_{\ell+\delta_{\ell}}\left(A+\alpha v v^{\top}\right) \leq \Phi_{\ell}(A)$ e a barreira atualizada $\ell+\delta_{\ell}$ continua limitando inferiormente os autovalores da matriz atualizada. Mostraremos também (no Lema 7.5) que, se $\alpha$ é limitado superiormente por $\left(v^{\top} U_{A}\left(u, \delta^{u}\right) v\right)^{-1}$ (onde $U_{A}\left(u, \delta^{u}\right)$ é uma matriz que depende de $A, u$ e do aumento $\delta^{u}$ da barreira superior e será definida a seguir), então $\Phi^{u+\delta^{u}}\left(A+\alpha v v^{\top}\right) \leq \Phi^{u}(A)$ e os autovalores da matriz atualizada se mantêm à esquerda da barreira superior atualizada $u+\delta^{u}$. Além disso, pelo Lema 7.6, dada uma coleção de vetores reais $V \operatorname{com} \sum_{v \in V} v v^{\top}=I$, existem um vetor $v \in V$ e um escalar $\alpha \in \mathbb{R}_{++}$, que é limitado inferior e superiormente por $\left(v^{\top} L_{A}\left(\ell, \delta_{\ell}\right) v\right)^{-1}$ e $\left(v^{\top} U_{A}\left(u, \delta_{u}\right) v\right)^{-1}$, respectivamente. Assim, pode-se obter uma matriz atualizada $A+\alpha v v^{\top}$ cujos potenciais (inferior e superior) são no máximo os potenciais de $A$ de forma que os autovalores das matrizes atualizadas permanecem entre as barreiras.

Definição 7.3. Seja $A \in \mathbb{R}^{n \times n}$ uma matriz simétrica e sejam $\ell \in \mathbb{R}, u, \delta^{u}, \delta_{\ell} \in \mathbb{R}_{++}$e $v \in \mathbb{R}^{n}$ tais que $\left(\ell+\delta_{\ell}\right) I \prec A \prec u I$. Denote

$$
\begin{aligned}
L_{A}\left(\ell, \delta_{\ell}\right) & :=\frac{\left(A-\ell^{\prime} I\right)^{-2}}{\Phi_{\ell^{\prime}}(A)-\Phi_{\ell}(A)}-\left(A-\ell^{\prime} I\right)^{-1}, \\
U_{A}\left(u, \delta^{u}\right) & :=\frac{\left(u^{\prime} I-A\right)^{-2}}{\Phi^{u}(A)-\Phi^{u^{\prime}}(A)}+\left(u^{\prime} I-A\right)^{-1} .
\end{aligned}
$$

onde $u^{\prime}:=u+\delta^{u}$ e $\ell^{\prime}:=\ell+\delta_{\ell}$. 
Lema 7.4. Seja $n \in \mathbb{N}$ com $n \geq 2$ e sejam $A \in \mathbb{R}^{n \times n}$ uma matriz simétrica, $v \in \mathbb{R}^{n} \backslash\{0\}, \ell \in \mathbb{R}$ e $\delta_{\ell}, \alpha \in \mathbb{R}_{++}$tais que $\lambda_{\min }(A)>\ell$ e $\Phi_{\ell}(A) \leq \frac{1}{\delta_{\ell}}$. Se

$$
0<\frac{1}{\alpha} \leq v^{\top} L_{A}\left(\ell, \delta_{\ell}\right) v
$$

então $\Phi_{\ell+\delta_{\ell}}\left(A+\alpha v v^{\top}\right) \leq \Phi_{\ell}(A)$ e $\lambda_{\min }\left(A+\alpha v v^{\top}\right)>\ell+\delta_{\ell}$.

Demonstração. Como $n \geq 2$, então

$$
\frac{1}{\lambda_{\min }(A)-\ell}<\Phi_{\ell}(A) \leq \frac{1}{\delta_{\ell}}
$$

o que implica que $\lambda_{\min }(A)>\ell+\delta_{\ell}$. Assim, vale que $\lambda_{\min }\left(A+\alpha v v^{\top}\right)>\ell+\delta_{\ell}=: \ell^{\prime}$ e que $\lambda_{i}^{\downarrow}\left(A-\ell^{\prime} I\right)=$ $\lambda_{i}^{\downarrow}(A)-\ell^{\prime}>0$ para todo $i \in[n]$. Logo, vale que $A-\ell^{\prime} I$ é positiva definida e, portanto, $A-\ell^{\prime} I$ é inversível. Note que $\left(A-\ell^{\prime} I\right)^{-1} \succ 0$, o que implica que $1+\alpha v\left(A-\ell^{\prime} I\right)^{-1} v^{\top} \geq 1$. Portanto, as hipóteses do Lema 2.17 se aplicam e temos

$$
\left(\left(A-\ell^{\prime} I\right)+\alpha v v^{\top}\right)^{-1}=\left(A-\ell^{\prime} I\right)^{-1}-\frac{\alpha\left(A-\ell^{\prime} I\right)^{-1} v v^{\top}\left(A-\ell^{\prime} I\right)^{-1}}{1+\alpha v^{\top}\left(A-\ell^{\prime} I\right)^{-1} v},
$$

o que implica que

$$
\begin{aligned}
\Phi_{\ell^{\prime}}\left(A+\alpha v v^{\top}\right) & =\operatorname{Tr}\left(\left(\left(A+\alpha v v^{\top}\right)-\ell^{\prime} I\right)^{-1}\right)=\operatorname{Tr}\left(\left(\left(A-\ell^{\prime} I\right)+\alpha v v^{\top}\right)^{-1}\right) \\
& =\operatorname{Tr}\left(\left(A-\ell^{\prime} I\right)^{-1}-\frac{\alpha\left(A-\ell^{\prime} I\right)^{-1} v v^{\top}\left(A-\ell^{\prime} I\right)^{-1}}{1+\alpha v^{\top}\left(A-\ell^{\prime} I\right)^{-1} v}\right) \\
& =\Phi_{\ell^{\prime}}(A)-\frac{\alpha \operatorname{Tr}\left(\left(\left(A-\ell^{\prime} I\right)^{-1} v\right)\left(v^{\top}\left(A-\ell^{\prime} I\right)^{-1}\right)\right)}{1+\alpha v^{\top}\left(A-\ell^{\prime} I\right)^{-1} v} \\
& =\Phi_{\ell^{\prime}}(A)-\frac{\alpha v^{\top}\left(A-\ell^{\prime} I\right)^{-2} v}{1+\alpha v^{\top}\left(A-\ell^{\prime} I\right)^{-1} v} \\
& \leq \Phi_{\ell^{\prime}}(A)-\frac{\alpha v^{\top}\left(A-\ell^{\prime} I\right)^{-2} v}{\frac{\alpha v^{\top}\left(A-\ell^{\prime} I\right)^{-2} v}{\Phi_{\ell^{\prime}}(A)-\Phi_{\ell}(A)}} \\
& =\Phi_{\ell^{\prime}}(A)-\left(\Phi_{\ell^{\prime}}(A)-\Phi_{\ell}(A)\right) \\
& =\Phi_{\ell}(A) .
\end{aligned}
$$

Lema 7.5. Sejam $A \in \mathbb{R}^{n \times n}$ uma matriz simétrica, $v \in \mathbb{R}^{n} \backslash\{0\}, u, \delta^{u} \in \mathbb{R}_{++}$e $\alpha \in \mathbb{R}_{++}$tais que $\lambda_{\max }(A)<u$. Se

$$
\frac{1}{\alpha} \geq v^{\top} U_{A}\left(u, \delta^{u}\right) v
$$

então $\Phi^{u+\delta^{u}}\left(A+\alpha v v^{\top}\right) \leq \Phi^{u}(A)$ e $\lambda_{\max }\left(A+\alpha v v^{\top}\right)<u+\delta^{u}$.

Demonstração. Defina $u^{\prime}:=u+\delta^{u}$. Note que $\lambda_{i}^{\downarrow}\left(u^{\prime} I-A\right)=u^{\prime}-\lambda_{i}^{\uparrow}(A)>0$ para todo $i \in[n]$. Logo, $u^{\prime} I-A \succ 0$, o que implica que $u^{\prime} I-A$ é inversível. Note também que, como $u^{\prime} I-A \succ 0$, vale que

$$
v^{\top}\left(u^{\prime} I-A\right)^{-2} v>0
$$


e também que

$$
\Phi^{u}(A)=\sum_{i \in[n]}\left(u-\lambda_{i}^{\downarrow}(A)\right)^{-1}>\sum_{i \in[n]}\left(u+\delta^{u}-\lambda_{i}^{\downarrow}(A)\right)^{-1}=\Phi^{u+\delta^{u}}(A) .
$$

Logo, vale que

$$
\Phi^{u}(A)-\Phi^{u^{\prime}}(A)=\Phi^{u}(A)-\Phi^{u+\delta^{u}}(A)>0 .
$$

Por (7.2) temos que

$$
1 \geq \frac{\alpha v^{\top}\left(u^{\prime} I-A\right)^{-2} v}{\Phi^{u}(A)-\Phi^{u^{\prime}}(A)}+\alpha v^{\top}\left(u^{\prime} I-A\right)^{-1} v .
$$

Dessa forma, por (7.3) e (7.4) temos que

$$
1-\alpha v^{\top}\left(u^{\prime} I-A\right)^{-1} v \geq \frac{\alpha v^{\top}\left(u^{\prime} I-A\right)^{-2} v}{\Phi^{u}(A)-\Phi^{u^{\prime}}(A)}>0 .
$$

Portanto, as hipóteses do Lema 2.17 se aplicam e temos

$$
\left(\left(u^{\prime} I-A\right)-\alpha v v^{\top}\right)^{-1}=\left(u^{\prime} I-A\right)^{-1}+\frac{\alpha\left(u^{\prime} I-A\right)^{-1} v v^{\top}\left(u^{\prime} I-A\right)^{-1}}{1-\alpha v^{\top}\left(u^{\prime} I-A\right)^{-1} v},
$$

o que implica que

$$
\begin{aligned}
\Phi^{u^{\prime}}\left(A+\alpha v v^{\top}\right) & =\operatorname{Tr}\left(\left(u^{\prime} I-\left(A+\alpha v v^{\top}\right)\right)^{-1}\right)=\operatorname{Tr}\left(\left(\left(u^{\prime} I-A\right)-\alpha v v^{\top}\right)^{-1}\right) \\
& =\operatorname{Tr}\left(\left(u^{\prime} I-A\right)^{-1}+\frac{\alpha\left(u^{\prime} I-A\right)^{-1} v v^{\top}\left(u^{\prime} I-A\right)^{-1}}{1-\alpha v^{\top}\left(u^{\prime} I-A\right)^{-1} v}\right) \\
& =\Phi^{u^{\prime}}(A)+\frac{\alpha \operatorname{Tr}\left(\left(u^{\prime} I-A\right)^{-1} v v^{\top}\left(u^{\prime} I-A\right)^{-1}\right)}{1-\alpha v^{\top}\left(u^{\prime} I-A\right)^{-1} v} \\
& =\Phi^{u^{\prime}}(A)+\frac{\alpha v^{\top}\left(u^{\prime} I-A\right)^{-2} v}{1-\alpha v^{\top}\left(u^{\prime} I-A\right)^{-1} v}
\end{aligned}
$$

Por (7.3) e (7.5),

$$
\begin{aligned}
\Phi^{u^{\prime}}\left(A+\alpha v v^{\top}\right) & \leq \Phi^{u^{\prime}}(A)+\alpha \frac{v^{\top}\left(u^{\prime} I-A\right)^{-2} v}{\frac{\alpha v^{\top}\left(u^{\prime} I-A\right)^{-2} v}{\Phi^{u}(A)-\Phi^{u^{\prime}}(A)}} \\
& =\Phi^{u^{\prime}}(A)+\Phi^{u}(A)-\Phi^{u^{\prime}}(A)=\Phi^{u}(A) .
\end{aligned}
$$

Suponha, por contradição, que $\lambda_{\max }\left(A+\alpha v v^{\top}\right) \geq u^{\prime}$. Seja $\varepsilon \in\left(0, u^{\prime}-\lambda_{\max }(A)\right)$ suficientemente pequeno para que $\frac{1}{\varepsilon}>\Phi^{u}(A)$. Note que $\lambda_{\max }\left(A+0 v v^{\top}\right)<u^{\prime}$. Assim, pelo Lema 4.18 e pelo Teorema do Valor Intermediário, existe $\alpha^{\prime} \in[0, \alpha]$ tal que $\lambda_{\max }\left(A+\alpha^{\prime} v v^{\top}\right)=u^{\prime}-\varepsilon$. Logo, vale que

$$
\Phi^{u^{\prime}}\left(A+\alpha^{\prime} v v^{\top}\right) \geq \frac{1}{u^{\prime}-\left(u^{\prime}-\varepsilon\right)}=\frac{1}{\varepsilon}>\Phi^{u}(A)
$$

Além disso, vale que

$$
\frac{1}{\alpha^{\prime}} \geq \frac{1}{\alpha} \geq v^{\top} U_{A}\left(u, \delta^{u}\right) v
$$

e, portanto, $\Phi^{u^{\prime}}\left(A+\alpha^{\prime} v v^{\top}\right) \leq \Phi^{u}(A)$, o que é uma contradição. 
Seja $V \subseteq \mathbb{R}^{n} \backslash\{0\}$ um conjunto de vetores com $\sum_{v \in V} v v^{\top}=I$. Mostraremos a seguir a existência de $\alpha \in \mathbb{R}_{++}$e $v \in V$, que satisfazem as condições dos Lemas 7.4 e 7.5. Assim, vale que os potenciais de algumas matrizes que sofrem determinadas atualizações de posto 1 não aumentam e que os autovalores dessas matrizes continuam sendo limitados pelas barreiras atualizadas.

Lema 7.6. Sejam $n \geq 2, A \in \mathbb{R}^{n \times n}$ uma matriz simétrica, $\ell \in \mathbb{R}$ e $u, \delta^{u}, \delta_{\ell} \in \mathbb{R}_{++}$tais que $\lambda_{\max }(A)<u$ e $\ell<\lambda_{\min }(A)$. Sejam $\phi_{\ell}, \phi^{u} \in \mathbb{R}_{++}$tais que $\Phi_{\ell}(A) \leq \phi_{\ell}$ e $\Phi^{u}(A) \leq \phi^{u}$ e seja $V \subseteq \mathbb{R}^{n} \backslash\{0\}$ tal que $\sum_{v \in V} v v^{\top}=I$. Se

$$
0<\frac{1}{\delta^{u}}+\phi^{u} \leq \frac{1}{\delta_{\ell}}-\phi_{\ell}
$$

então existem $v \in V$ e $\alpha \in \mathbb{R}_{++}$tais que

$$
v^{\top} U_{A}\left(u, \delta^{u}\right) v \leq \frac{1}{\alpha} \leq v^{\top} L_{A}\left(\ell, \delta_{\ell}\right) v
$$

Demonstração. Defina $u^{\prime}:=u+\delta^{u}$ e $\ell^{\prime}:=\ell+\delta_{\ell}$. Note que para todo $i \in[n]$,

$$
\left(u-\lambda_{i}^{\downarrow}(A)\right)^{-1}>\left(u^{\prime}-\lambda_{i}^{\downarrow}(A)\right)^{-1}>0 .
$$

Note que

$$
\begin{aligned}
\sum_{v \in V} v^{\top} U_{A}\left(u, \delta^{u}\right) v & =\sum_{v \in V} \operatorname{Tr}\left(v^{\top} U_{A}\left(u, \delta^{u}\right) v\right) \\
& =\sum_{v \in V} \operatorname{Tr}\left(U_{A}\left(u, \delta^{u}\right) v v^{\top}\right) \text { pela Proposição } 2.15 \\
& =\operatorname{Tr}\left(U_{A}\left(u, \delta^{u}\right) \sum_{v \in V} v v^{\top}\right) \\
& =\operatorname{Tr}\left(U_{A}\left(u, \delta^{u}\right)\right) .
\end{aligned}
$$

Dessa forma, vale que

$$
\begin{aligned}
\sum_{v \in V} v^{\top} U_{A}\left(u, \delta^{u}\right) v & =\frac{\operatorname{Tr}\left(\left(u^{\prime} I-A\right)^{-2}\right)}{\Phi^{u}(A)-\Phi^{u^{\prime}}(A)}+\operatorname{Tr}\left(\left(u^{\prime} I-A\right)^{-1}\right) \\
& =\frac{\sum_{i \in[n]} \lambda_{i}^{\downarrow}\left(\left(u^{\prime} I-A\right)^{-2}\right)}{\Phi^{u}(A)-\Phi^{u^{\prime}}(A)}+\Phi^{u^{\prime}}(A) \\
& =\frac{\sum_{i \in[n]}\left(u^{\prime}-\lambda_{i}^{\downarrow}(A)\right)^{-2}}{\sum_{i \in[n]}\left(\frac{1}{u-\lambda_{i}^{\downarrow}(A)}-\frac{1}{u^{\prime}-\lambda_{i}^{\downarrow}(A)}\right)}+\Phi^{u^{\prime}}(A) \\
& =\frac{\sum_{i \in[n]}\left(u^{\prime}-\lambda_{i}^{\downarrow}(A)\right)^{-2}}{\delta^{u} \sum_{i \in[n]}\left(u-\lambda_{i}^{\downarrow}(A)\right)^{-1}\left(u^{\prime}-\lambda_{i}^{\downarrow}(A)\right)^{-1}}+\Phi^{u^{\prime}}(A) \\
& \leq \frac{\sum_{i \in[n]}\left(u^{\prime}-\lambda_{i}^{\downarrow}(A)\right)^{-2}}{\delta^{u} \sum_{i \in[n]}\left(u^{\prime}-\lambda_{i}^{\downarrow}(A)\right)^{-2}}+\Phi^{u^{\prime}}(A) \\
& =\frac{1}{\delta^{u}+\Phi^{u^{\prime}}(A) \leq \frac{1}{\delta^{u}}+\phi^{u} .}
\end{aligned}
$$


Por Cauchy-Schwarz (Lema 2.16), temos que

$$
\begin{aligned}
\left(\delta _ { \ell } \sum _ { i \in [ n ] } \left(\lambda_{i}^{\downarrow}(A)\right.\right. & \left.\left.-\ell^{\prime}\right)^{-1}\left(\lambda_{i}^{\downarrow}(A)-\ell\right)^{-1}\right)^{2} \\
& =\left(\sum_{i \in[n]} \delta_{\ell}^{\frac{1}{2}}\left(\lambda_{i}^{\downarrow}(A)-\ell^{\prime}\right)^{-1}\left(\lambda_{i}^{\downarrow}(A)-\ell\right)^{-\frac{1}{2}} \delta_{\ell}^{\frac{1}{2}}\left(\lambda_{i}^{\downarrow}(A)-\ell\right)^{-\frac{1}{2}}\right)^{2} \\
& \leq\left(\delta_{\ell} \sum_{i \in[n]}\left(\lambda_{i}^{\downarrow}(A)-\ell^{\prime}\right)^{-2}\left(\lambda_{i}^{\downarrow}(A)-\ell\right)^{-1}\right)\left(\delta_{\ell} \sum_{i \in[n]}\left(\lambda_{i}^{\downarrow}(A)-\ell\right)^{-1}\right) \\
& =\left(\delta_{\ell} \sum_{i \in[n]}\left(\lambda_{i}^{\downarrow}(A)-\ell^{\prime}\right)^{-2}\left(\lambda_{i}^{\downarrow}(A)-\ell\right)^{-1}\right)\left(\delta_{\ell} \Phi_{\ell}(A)\right) \\
& \leq\left(\delta_{\ell} \sum_{i \in[n]}\left(\lambda_{i}^{\downarrow}(A)-\ell^{\prime}\right)^{-2}\left(\lambda_{i}^{\downarrow}(A)-\ell\right)^{-1}\right)\left(\delta_{\ell} \phi_{\ell}\right) \\
& \leq \delta_{\ell} \sum_{i \in[n]}\left(\lambda_{i}^{\downarrow}(A)-\ell^{\prime}\right)^{-2}\left(\lambda_{i}^{\downarrow}(A)-\ell\right)^{-1} \\
& =\sum_{i \in[n]}\left(\lambda_{i}^{\downarrow}(A)-\ell^{\prime}\right)^{-2}-\sum_{i \in[n]}\left(\lambda_{i}^{\downarrow}(A)-\ell^{\prime}\right)^{-1}\left(\lambda_{i}^{\downarrow}(A)-\ell\right)^{-1},
\end{aligned}
$$

o que implica que

$$
\begin{aligned}
\sum_{i \in[n]} & \left(\lambda_{i}^{\downarrow}(A)-\ell^{\prime}\right)^{-2} \\
& \geq \sum_{i \in[n]}\left(\lambda_{i}^{\downarrow}(A)-\ell^{\prime}\right)^{-1}\left(\lambda_{i}^{\downarrow}(A)-\ell\right)^{-1}+\left(\delta_{\ell} \sum_{i \in[n]}\left(\lambda_{i}^{\downarrow}(A)-\ell^{\prime}\right)^{-1}\left(\lambda_{i}^{\downarrow}(A)-\ell\right)^{-1}\right)^{2} \\
& =\left(\delta_{\ell} \sum_{i \in[n]}\left(\lambda_{i}^{\downarrow}(A)-\ell^{\prime}\right)^{-1}\left(\lambda_{i}^{\downarrow}(A)-\ell\right)^{-1}\right)\left(\frac{1}{\delta_{\ell}}+\delta_{\ell} \sum_{i \in[n]}\left(\lambda_{i}^{\downarrow}(A)-\ell^{\prime}\right)^{-1}\left(\lambda_{i}^{\downarrow}(A)-\ell\right)^{-1}\right) \\
& =\left(\sum_{i \in[n]}\left(\lambda_{i}^{\downarrow}(A)-\ell^{\prime}\right)^{-1}-\sum_{i \in[n]}\left(\lambda_{i}^{\downarrow}(A)-\ell\right)^{-1}\right)\left(\frac{1}{\delta_{\ell}}+\sum_{i \in[n]}\left(\lambda_{i}^{\downarrow}(A)-\ell^{\prime}\right)^{-1}-\sum_{i \in[n]}\left(\lambda_{i}^{\downarrow}(A)-\ell\right)^{-1}\right) \\
& =\left(\Phi_{\ell^{\prime}}(A)-\Phi_{\ell}(A)\right)\left(\frac{1}{\delta_{\ell}}+\Phi_{\ell^{\prime}}(A)-\Phi_{\ell}(A)\right) .
\end{aligned}
$$

Logo, temos que

$$
\frac{\sum_{i \in[n]}\left(\lambda_{i}^{\downarrow}(A)-\ell^{\prime}\right)^{-2}}{\Phi_{\ell^{\prime}}(A)-\Phi_{\ell}(A)} \geq \frac{1}{\delta_{\ell}}+\Phi_{\ell^{\prime}}(A)-\Phi_{\ell}(A) .
$$

Note também que

$$
\begin{array}{rlr}
\sum_{v \in V} v^{\top} L_{A}\left(\ell, \delta_{\ell}\right) v & =\sum_{v \in V} \operatorname{Tr}\left(v^{\top} L_{A}\left(\ell, \delta_{\ell}\right) v\right) & \\
& =\sum_{v \in V} \operatorname{Tr}\left(L_{A}\left(\ell, \delta_{\ell}\right) v v^{\top}\right) & \text { pela Proposição } 2.15 \\
& =\operatorname{Tr}\left(L_{A}\left(\ell, \delta_{\ell}\right)\right) \\
& =\frac{\operatorname{Tr}\left(A-\ell^{\prime} I\right)^{-2}}{\Phi_{\ell^{\prime}}(A)-\Phi_{\ell}(A)}-\operatorname{Tr}\left(A-\ell^{\prime} I\right)^{-1}
\end{array}
$$




$$
\begin{aligned}
& =\frac{\sum_{i \in[n]} \lambda_{i}^{\downarrow}\left(A-\ell^{\prime} I\right)^{-2}}{\Phi_{\ell^{\prime}}(A)-\Phi_{\ell}(A)}-\Phi_{\ell^{\prime}}(A) \\
& =\frac{\sum_{i \in[n]}\left(\lambda_{i}^{\downarrow}(A)-\ell^{\prime}\right)^{-2}}{\Phi_{\ell^{\prime}}(A)-\Phi_{\ell}(A)}-\Phi_{\ell^{\prime}}(A) \\
& \begin{array}{ll}
\geq \frac{1}{\delta_{\ell}}-\Phi_{\ell}(A) & \text { por }(7.8)
\end{array} \\
& \geq \frac{1}{\delta_{\ell}}-\phi_{\ell}
\end{aligned}
$$

Logo, temos que

$$
\sum_{v \in V} v^{\top} U_{A}\left(u, \delta^{u}\right) v \leq \frac{1}{\delta^{u}}+\phi^{u} \leq \frac{1}{\delta_{\ell}}-\phi_{\ell} \leq \sum_{v \in V} v^{\top} L_{A}\left(\ell, \delta_{\ell}\right) v
$$

o que implica que existe $v \in V$ tal que $v^{\top} U_{A}\left(u, \delta^{u}\right) v \leq v^{\top} L_{A}\left(\ell, \delta_{\ell}\right) v$ e, como $\frac{1}{\delta^{u}}+\phi^{u}>0$, então existe $\alpha \in \mathbb{R}_{++}$tal que

$$
v^{\top} U_{A}\left(u, \delta^{u}\right) v \leq \frac{1}{\alpha} \leq v^{\top} L_{A}\left(\ell, \delta_{\ell}\right) v
$$

\subsection{Construção de expansores irregulares}

Nesta seção, utilizaremos os resultados de barreiras e funções potenciais, apresentados na seção anterior, para construir bons esparsificadores espectrais de grafos arbitrários e, mais especificamente, grafos expansores a partir de grafos completos. Seja $V$ uma coleção de vetores reais com $\sum_{v \in V} v v^{\top}=I$. Primeiramente, utilizamos os resultados de barreiras e funções potenciais para mostrar que é possível construir matrizes que se aproximam da identidade (autovalores em $[1,1+\varepsilon]$, com $\varepsilon>0$ próximo de 0 ) realizando sucessivas atualizações de posto 1 com vetores de $V$. Esse resultado pode ser provado de forma construtiva, de modo que, a cada iteração, seja feita uma atualização de posto 1 em uma matriz que é inicialmente 0 , até que essa matriz se aproxime da matriz identidade. Mantemos barreiras inferiores e superiores (que são aumentadas de uma constante a cada iteração) para cada atualização de posto 1 realizada, de forma que os autovalores de cada matriz estejam entre as barreiras associadas a essa iteração.

Mais adiante, mostraremos que a construção de um esparsificador espectral $H$ que $(1+\varepsilon)$ aproxima o grafo completo $G$ (com $\varepsilon>0$ próximo de 0 ) pode ser reduzida para a construção de matrizes formadas por atualizações de posto 1 que se aproximam da matriz identidade.

Teorema 7.7 (Batson, Spielman e Srivastava [2]). Sejam $d \in \mathbb{R}$ com $d>1$ e $U$ um conjunto finito com $|U| \geq 2$. Seja $V \subseteq \mathbb{R}^{U} \backslash\{0\}$ finito tal que

$$
\sum_{v \in V} v v^{\top}=I
$$

Então existe uma função $s: V \rightarrow \mathbb{R}_{+} \operatorname{com}|\operatorname{supp}(s)| \leq d|U|$ tal que

$$
I \preceq \sum_{v \in V} s_{v} v v^{\top} \preceq\left(\frac{d+1+2 \sqrt{d}}{d+1-2 \sqrt{d}}\right) I .
$$

Demonstração. Seja $n:=|U|$ e sejam

$$
\ell_{0}:=-n \sqrt{d}, \quad u_{0}:=\frac{n(d+\sqrt{d})}{\sqrt{d}-1},
$$




$$
\delta_{\ell}:=1, \quad \delta^{u}:=\frac{\sqrt{d}+1}{\sqrt{d}-1}
$$

Note que $\Phi_{\ell_{0}}(0)=\frac{1}{\sqrt{d}}$ e $\Phi^{u_{0}}(0)=\frac{\sqrt{d}-1}{d+\sqrt{d}}$. Denote $\phi_{\ell}:=\Phi_{\ell_{0}}(0)$ e $\phi^{u}:=\Phi^{u_{0}}(0)$.

Provaremos, por indução em $k$, que existem funções $s: \mathbb{N} \rightarrow \mathbb{R}_{+}$e $\omega: \mathbb{N} \rightarrow V$ tais que

$$
\begin{aligned}
& \lambda_{\min }\left(A_{(k)}\right)>\ell_{0}+k \delta_{\ell} \text { e } \lambda_{\max }\left(A_{(k)}\right)<u_{0}+k \delta^{u}, \\
& \Phi_{\ell_{0}+k \delta_{\ell}}\left(A_{(k)}\right) \leq \phi_{\ell} \text { e } \Phi_{u_{0}+k \delta^{u}}\left(A_{(k)}\right) \leq \phi^{u}
\end{aligned}
$$

para todo $k \in \mathbb{N}$, onde $A_{(k)}:=\sum_{j \in[k]} s_{j} \omega_{j} \omega_{j}^{\top}$ para todo $k \in \mathbb{N}$.

É fácil ver que o resultado vale se $k=0$.

Suponha que $k \geq 1$. Pela hipótese de indução, temos que

$$
\begin{gathered}
\lambda_{\min }\left(A_{(k-1)}\right)>\ell_{0}+(k-1) \delta_{\ell} \\
\text { e } \quad \lambda_{\max }\left(A_{(k-1)}\right)<u_{0}+(k-1) \delta^{u}, \\
\Phi_{\ell_{0}+(k-1) \delta_{\ell}}\left(A_{(k-1)}\right) \leq \phi_{\ell} \text { e } \Phi_{u_{0}+(k-1) \delta^{u}}\left(A_{(k-1)}\right) \leq \phi^{u} .
\end{gathered}
$$

Note que

$$
0<\frac{1}{\delta^{u}}+\phi^{u}=\frac{\sqrt{d}-1}{\sqrt{d}+1}+\frac{\sqrt{d}-1}{\sqrt{d}+d}=\frac{\sqrt{d}-1}{\sqrt{d}}=\frac{1}{\delta_{\ell}}-\phi_{\ell},
$$

o que implica que as hipóteses do Lema 7.6 são satisfeitas e, portanto, as hipóteses dos Lemas 7.4 e 7.5 se aplicam e podemos concluir que existem $v \in V$ e $\alpha \in \mathbb{R}_{++}$tais que

$$
\begin{aligned}
& \lambda_{\min }\left(A_{(k-1)}+\alpha v v^{\top}\right)>\ell_{0}+k \delta_{\ell} \text { e } \lambda_{\max }\left(A_{(k-1)}+\alpha v v^{\top}\right)<u_{0}+k \delta^{u}, \\
& \Phi_{\ell_{0}+k \delta_{\ell}}\left(A_{(k-1)}+\alpha v v^{\top}\right) \leq \Phi_{\ell_{0}+(k-1) \delta_{\ell}}\left(A_{(k-1)}\right) \text { e } \Phi_{u_{0}+k \delta^{u}}\left(A_{(k-1)}+\alpha v v^{\top}\right) \leq \Phi_{u_{0}+(k-1) \delta^{u}}\left(A_{(k-1)}\right) \text {. }
\end{aligned}
$$

Tome $s_{k}:=\alpha$ e $\omega_{k}:=v$. Note que

$$
\begin{aligned}
& \lambda_{\min }\left(A_{(k)}\right)>\ell_{0}+k \delta_{\ell} \text { e } \lambda_{\max }\left(A_{(k)}\right)<u_{0}+k \delta^{u}, \\
& \Phi_{\ell_{0}+k \delta_{\ell}}\left(A_{(k)}\right) \leq \phi_{\ell} \text { e } \Phi_{u_{0}+k \delta^{u}}\left(A_{(k)}\right) \leq \phi^{u} .
\end{aligned}
$$

Defina $q:=d n$. Com base no resultado provado anteriormente

$$
\lambda_{\min }\left(A_{(q)}\right)>\ell_{0}+q \delta_{\ell}>0 \text { e } \lambda_{\max }\left(A_{(q)}\right)<u_{0}+q \delta^{u},
$$

o que implica que

$$
\frac{\lambda_{\max }\left(A_{(q)}\right)}{\lambda_{\min }\left(A_{(q)}\right)}<\frac{u_{0}+d n \delta^{u}}{\ell_{0}+d n \delta_{\ell}}=\frac{d+1+2 \sqrt{d}}{d+1-2 \sqrt{d}} .
$$

Portanto, como $A_{(q)} \succ 0$, então

$$
I \preceq \frac{1}{\lambda_{\min }\left(A_{(q)}\right)} A_{(q)} \preceq\left(\frac{d+1+2 \sqrt{d}}{d+1-2 \sqrt{d}}\right) I .
$$

Dessa forma, existe $s: V \mapsto \mathbb{R}_{+}$tal que

$$
\frac{1}{\lambda_{\min }\left(A_{(q)}\right)} A_{(q)}=\sum_{v \in V} s_{v} v v^{\top}
$$

Portanto, vale que

$$
I \preceq \sum_{v \in V} s_{v} v v^{\top} \preceq\left(\frac{d+1+2 \sqrt{d}}{d+1-2 \sqrt{d}}\right) I .
$$


A contribuição da prova do Teorema 7.7 na construção de esparsificadores espectrais é a criação da função $s: V \rightarrow \mathbb{R}_{+}$com o uso de barreiras. A função $s$ é utilizada para atribuir pesos às arestas do esparsificador espectral, de modo que cada elemento do domínio de $s$ seja representado por uma aresta do esparsificador. Assim, o peso atribuído a cada aresta $e$ do esparsificador é $s\left(v_{e}\right)$, onde $v_{e} \in V$ é o vetor que representa a aresta $e$.

Discutiremos brevemente o algoritmo baseado na prova do Teorema 7.7, que cria essa função $s$. Para obter essa função, definimos inicialmente a matriz $A_{0}:=0$ e chamamos de $A_{i}$ a matriz referente a $i$-ésima iteração desse algoritmo. Feito isso, determinam-se duas barreiras iniciais próximas, que limitam inferior e superiormente os autovalores de $A_{0}$. Também são definidas duas constantes positivas, que são somadas às barreiras, e assim ditam o quanto as barreiras se movem a cada iteração. Assim, os limitantes inferior e superior marcham de forma constante a cada iteração, e uma escolha de $A_{i}$ permite que todos os autovalores de $A_{i}$ se mantenham entre as barreiras referentes à $i$-ésima iteração. A prova construtiva do Teorema 7.7 fornece, a cada iteração $i$, um vetor $v \in V$ e um número real positivo $\alpha_{i}$ tais que todos os autovalores da matriz $A_{i}:=A_{i-1}+\alpha_{i} v v^{\top}$ estão entre as duas barreiras em progresso. Assim, os autovalores das matrizes em progresso serão limitados inferior e superiormente pelas suas respectivas barreiras e, além disso, os autovalores dessas matrizes são impedidos de ficarem próximos demais dessas barreiras, que são assim chamadas por esse motivo. Desta forma, controlamos os autovalores das matrizes em progresso de forma que, em um número linear de iterações, (7.10) é satisfeito.

Como utilizamos Laplacianos de grafos (que satisfazem $\operatorname{ker}\left(L_{G}\right) \neq\{0\}$ ), estamos interessados em resultados que utilizam projetores ortogonais em vez de matrizes identidade. Por esse motivo, generalizamos o resultado apresentado anteriormente, utilizando projetores ortogonais no lugar de matrizes identidade.

Corolário 7.8. Sejam $d \in \mathbb{R}$ com $d>1, U$ um conjunto finito com $|U| \geq 2$ e $W$ um subespaço vetorial de $\mathbb{R}^{U}$. Seja $V \subseteq W \backslash\{0\}$ finito tal que

$$
\sum_{v \in V} v v^{\top}=P_{W}
$$

Então existe uma função $s: V \rightarrow \mathbb{R}_{+} \operatorname{com}|\operatorname{supp}(s)| \leq d \operatorname{dim}(W)$ tal que

$$
P_{W} \preceq \sum_{v \in V} s_{v} v v^{\top} \preceq\left(\frac{d+1+2 \sqrt{d}}{d+1-2 \sqrt{d}}\right) P_{W} .
$$

Demonstração. Defina $n:=|U|$ e $m:=\operatorname{dim}(W)$. Para simplificar a notação, trataremos $U$ como $[n]$. Sejam $\left\{u_{1}, \ldots, u_{m}\right\}$ uma base ortonormal de $W$ e $\left\{u_{m+1}, \ldots, u_{n}\right\}$ uma base ortonormal de $W^{\perp}$. Note que $\left\{u_{1}, \ldots, u_{n}\right\}$ é uma base ortonormal de $\mathbb{R}^{n}$. Note também que

$$
\sum_{i \in[m]} u_{i} u_{i}^{\top}=P_{W}=\sum_{v \in V} v v^{\top}
$$

Seja $M \in \mathbb{R}^{n \times n}$ tal que $M e_{i}=u_{i}$ para todo $i \in[n]$. Defina a função $f: V \rightarrow \mathbb{R}^{m}$ como $v \mapsto M^{\top} v$. Como $V \subseteq W$, então vale que $f(v)_{i}=0$ para todo $v \in V$ e todo $i \in\{m+1, \ldots, n\}$. Além disso, vale que

$$
M^{\top} P_{W} M=M^{\top} \sum_{i \in[m]}\left(u_{i} u_{i}^{\top}\right) M=\sum_{i \in[m]}\left(M^{\top} u_{i} u_{i}^{\top} M\right)=\sum_{i \in[m]}\left(e_{i} e_{i}^{\top}\right) .
$$


Logo, pelo Teorema 7.7, temos que existe uma função $s: f(V) \rightarrow \mathbb{R}_{+} \operatorname{com}|\operatorname{supp}(s)| \leq d m$ tal que

$$
\sum_{i \in[m]}\left(e_{i} e_{i}^{\top}\right) \preceq \sum_{v \in f(V)} s_{v} v v^{\top} \preceq\left(\frac{d+1+2 \sqrt{d}}{d+1-2 \sqrt{d}}\right) \sum_{i \in[m]}\left(e_{i} e_{i}^{\top}\right),
$$

o que implica que

$$
M^{\top} P_{W} M \preceq M^{\top} \sum_{v \in V}\left(s_{f(v)} v v^{\top}\right) M \preceq\left(\frac{d+1+2 \sqrt{d}}{d+1-2 \sqrt{d}}\right) M^{\top} P_{W} M .
$$

Seja $u \in \mathbb{R}^{n} \backslash\{0\}$. Como $\operatorname{rank}(M)=n$, então existe $\bar{u} \in \mathbb{R}^{n} \backslash\{0\}$ tal que $M \bar{u}=u$. Assim, como

$$
\bar{u}^{\top} M^{\top} P_{W} M \bar{u} \leq \bar{u}^{\top} M^{\top} \sum_{v \in V}\left(s_{f(v)} v v^{\top}\right) M \bar{u} \leq\left(\frac{d+1+2 \sqrt{d}}{d+1-2 \sqrt{d}}\right) \bar{u}^{\top} M^{\top} P_{W} M \bar{u},
$$

então

$$
u^{\top} P_{W} u \leq u^{\top} \sum_{v \in V}\left(s_{f(v)} v v^{\top}\right) u \leq\left(\frac{d+1+2 \sqrt{d}}{d+1-2 \sqrt{d}}\right) u^{\top} P_{W} u
$$

Logo, vale que

$$
P_{W} \preceq \sum_{v \in V}\left(s_{f(v)} v v^{\top}\right) \preceq\left(\frac{d+1+2 \sqrt{d}}{d+1-2 \sqrt{d}}\right) P_{W} .
$$

Com esse resultado, é possível construir um esparsificador espectral de um grafo com pesos nas arestas $G=(V, E, \mathbb{1})$ com um número linear de arestas. Primeiramente, associa-se as arestas de $G$ a um subconjunto $W \subseteq \mathbb{R}^{U} \backslash\{0\}$ e, a partir da construção de uma função $s: W \rightarrow \mathbb{R}_{+}$que satisfaz as propriedades apresentadas no Corolário 7.8, pode-se construir um esparsificador espectral desejado, aresta por aresta, atribuindo pesos às arestas do esparsificador em progresso de acordo com a função $s$.

Seja $G=\left(V, E, n(d-2 \sqrt{d-1})^{-1} \mathbb{1}\right)$ um grafo de Ramanujan $d$-regular não-bipartido com pesos nas arestas e $n$ vértices. Seja $K=\left(V,\left(\begin{array}{c}V \\ 2\end{array}\right), \mathbb{1}\right)$ um grafo completo. Tome $\beta:=n(d-2 \sqrt{d-1})^{-1}$. Note que

$$
L_{K}=n\left(I-\frac{1}{n} J\right)=n P_{\{\mathbb{1}\}^{\perp}}=\beta(d-2 \sqrt{d-1}) P_{\{\mathbb{1}\}^{\perp}} .
$$

Os autovalores não-nulos do Laplaciano de todo grafo de Ramanujan $d^{\prime}$-regular não-bipartido com peso $\mathbb{1}$ nas arestas são limitados inferior e superiormente por $d^{\prime}-2 \sqrt{d^{\prime}-1}$ e $d^{\prime}+2 \sqrt{d^{\prime}-1}$, respectivamente. Assim, como $G$ é um grafo de Ramanujan não-bipartido com peso $\beta \mathbb{1}$ nas arestas, então

$$
\beta(d-2 \sqrt{d-1}) P_{\{\mathbb{1}\}^{\perp}} \preceq L_{G} \preceq \beta(d+2 \sqrt{d-1}) P_{\{\mathbb{1}\}^{\perp}},
$$

o que implica que

$$
L_{K} \preceq L_{G} \preceq\left(\frac{d+2 \sqrt{d-1}}{d-2 \sqrt{d-1}}\right) L_{K} .
$$

Portanto, $G$ é uma $\left(\frac{d+2 \sqrt{d-1}}{d-2 \sqrt{d-1}}\right)$-aproximação de $K$.

Batson, Spielman e Srivastava mostram em [2] a existência de esparsificadores espectrais de grafos arbitrários que são $\left(\frac{d+1+2 \sqrt{d}}{d+1-2 \sqrt{d}}\right)$-aproximações de grafos completos. Como

$$
\frac{d+1+2 \sqrt{d}}{d+1-2 \sqrt{d}}<\frac{d+2 \sqrt{d-1}}{d-2 \sqrt{d-1}}
$$


para todo $d \in \mathbb{R}$ com $d \geq 3$, então vale que esses esparsificadores espectrais são tão bons quanto aproximações de grafos completos por grafos de Ramanujan não-bipartidos.

Para provar esse resultado, utilizamos pseudo-inversas de matrizes e alguns resultados apresentados anteriormente.

Teorema 7.9 (Batson, Spielman e Srivastava [2]). Sejam $d \in \mathbb{R}$ com $d>2$ e $G=(V, E, w)$ um grafo conexo com pesos nas arestas e com $n \geq 2$ vértices. Então existe um grafo com pesos nas arestas $H=(V, F, \bar{w})$ tal que $H$ é um subgrafo de $G$ com $|F| \leq d(n-1)$ e

$$
L_{G} \preceq L_{H} \preceq\left(\frac{d+1+2 \sqrt{d}}{d+1-2 \sqrt{d}}\right) L_{G} .
$$

Demonstração. Seja $\vec{G}$ uma orientação de $G$ e $B_{\vec{G}}$ a matriz de incidência de $\vec{G}$. Seja $W:=\operatorname{Diag}(w)$. Note que $\left(L_{G}^{+}\right)^{\frac{1}{2}}\left(L_{G}^{+}\right)^{\frac{1}{2}}=\left(L_{G}^{+}\right)$, que $\left(L_{G}\right)^{\frac{1}{2}}\left(L_{G}\right)^{\frac{1}{2}}=L_{G}$, e também que $\left(L_{G}^{+}\right)^{\frac{1}{2}}$ é a pseudo-inversa de $\left(L_{G}\right)^{\frac{1}{2}}$. Defina

$$
Y:=\left(L_{G}^{+}\right)^{\frac{1}{2}} B_{\vec{G}}^{\top} W^{\frac{1}{2}} \in \mathbb{R}^{V \times E}
$$

e $v_{i}:=Y e_{i}$ para todo $i \in E(G)$.

Note que

$$
\begin{aligned}
\sum_{i \in E(G)} v_{i} v_{i}^{\top} & =\sum_{i \in E(G)} Y e_{i} e_{i}^{\top} Y^{\top}=Y\left(\sum_{i \in E(G)} e_{i} e_{i}^{\top}\right) Y^{\top} \\
& =Y I Y^{\top}=Y Y^{\top} \\
& =\left(L_{G}^{+}\right)^{\frac{1}{2}} B_{\vec{G}}^{\top} W^{\frac{1}{2}}\left(W^{\frac{1}{2}}\right)^{\top} B_{\vec{G}}\left(\left(L_{G}^{+}\right)^{\frac{1}{2}}\right)^{\top} \\
& =\left(L_{G}^{+}\right)^{\frac{1}{2}} B_{\vec{G}}^{\top} W B_{\vec{G}}\left(L_{G}^{+}\right)^{\frac{1}{2}} \\
& =\left(L_{G}^{+}\right)^{\frac{1}{2}} L_{G}\left(L_{G}^{+}\right)^{\frac{1}{2}} \\
& =\left(L_{G}^{+}\right)^{\frac{1}{2}}\left(L_{G}\right)^{\frac{1}{2}}\left(L_{G}\right)^{\frac{1}{2}}\left(L_{G}^{+}\right)^{\frac{1}{2}} \\
& =\left(L_{G}\right)^{\frac{1}{2}}\left(L_{G}\right)^{\frac{1}{2}}\left(L_{G}^{+}\right)^{\frac{1}{2}}\left(L_{G}^{+}\right)^{\frac{1}{2}} \\
& =L_{G} L_{G}^{+} \\
& =P_{\operatorname{Im}\left(L_{G}\right)}
\end{aligned}
$$

pela Proposição 2.44

pela Proposição 2.44 .

Portanto, $d$ e $\left\{v_{i}: i \in E(G)\right\}$ satisfazem as condições do Corolário 7.8, o que implica que existe uma função $s: E(G) \mapsto \mathbb{R}_{+} \operatorname{com}|\operatorname{supp}(s)| \leq d(n-1)$ tal que

$$
P_{\operatorname{Im}\left(L_{G}\right)} \preceq \sum_{i \in E(G)} s_{i} v_{i} v_{i}^{\top}=Y S Y^{\top} \preceq k P_{\operatorname{Im}\left(L_{G}\right)},
$$

onde $S:=\operatorname{Diag}(s)$ e $k:=\frac{d+1+2 \sqrt{d}}{d+1-2 \sqrt{d}}$.

Pelo Lema 2.30, temos que

$$
1 \leq \frac{y^{\top} Y S Y^{\top} y}{y^{\top} y} \leq k
$$

para todo $y \in \operatorname{Im}\left(L_{G}\right) \backslash\{0\}$. Note que $\operatorname{Im}\left(L_{G}\right)=\operatorname{Im}\left(\left(L_{G}\right)^{\frac{1}{2}}\right)$, o que implica que

$$
1 \leq \frac{y^{\top} Y S Y^{\top} y}{y^{\top} y}=\frac{y^{\top}\left(L_{G}^{+}\right)^{\frac{1}{2}} B_{\vec{G}}^{\top} W^{\frac{1}{2}} S W^{\frac{1}{2}} B_{\vec{G}}\left(L_{G}^{+}\right)^{\frac{1}{2}} y}{y^{\top} y} \leq k
$$


para todo $y \in \operatorname{Im}\left(\left(L_{G}\right)^{\frac{1}{2}}\right) \backslash\{0\}$.

Defina $H:=\left(V, F, w^{\prime}\right)$ como um grafo com pesos nas arestas com $w^{\prime}:=\operatorname{Diag}(w) s$ e defina $F:=\operatorname{supp}\left(w^{\prime}\right)$. Note que $L_{H}=B_{\vec{G}}^{\top} W^{\frac{1}{2}} S W^{\frac{1}{2}} B_{\vec{G}}$. Note também que $H$ é um subgrafo de $G$ e que $|F| \leq d(n-1)$, pois $S$ é uma matriz diagonal com no máximo $d(n-1)$ elementos não nulos. Portanto,

$$
1 \leq \frac{y^{\top}\left(L_{G}^{+}\right)^{\frac{1}{2}} L_{H}\left(L_{G}^{+}\right)^{\frac{1}{2}} y}{y^{\top} y} \leq k
$$

para todo $y \in \operatorname{Im}\left(\left(L_{G}\right)^{\frac{1}{2}}\right) \backslash\{0\}$. Por hipótese, $G$ é conexo, o que implica que $\operatorname{ker}\left(L_{G}\right)=\operatorname{span}(\mathbb{1})$. Logo, vale que

$$
1 \leq \frac{x^{\top}\left(L_{G}\right)^{\frac{1}{2}}\left(L_{G}^{+}\right)^{\frac{1}{2}} L_{H}\left(L_{G}^{+}\right)^{\frac{1}{2}}\left(L_{G}\right)^{\frac{1}{2}} x}{x^{\top}\left(L_{G}\right)^{\frac{1}{2}}\left(L_{G}\right)^{\frac{1}{2}} x}=\frac{x^{\top} P_{\operatorname{Im}\left(L_{G}\right)} L_{H} P_{\operatorname{Im}\left(L_{G}\right)} x}{x^{\top} L_{G} x}=\frac{x^{\top} L_{H} x}{x^{\top} L_{G} x} \leq k
$$

para todo $x \in\{\mathbb{1}\}^{\perp} \backslash\{0\}=\operatorname{Im}\left(L_{G}\right) \backslash\{0\}$. Portanto,

$$
x^{\top} L_{G} x \leq x^{\top} L_{H} x \leq\left(\frac{d+1+2 \sqrt{d}}{d+1-2 \sqrt{d}}\right) x^{\top} L_{G} x
$$

para todo $x \in \mathbb{R}^{V}$.

Seja $G=(V, E, w)$ um grafo conexo com pesos nas arestas e com $n$ vértices. Para construir um esparsificador espectral que é uma $\left(\frac{d+1+2 \sqrt{d}}{d+1-2 \sqrt{d}}\right)$-aproximação de $G$, construímos um subconjunto $U \subseteq \mathbb{R}^{V} \backslash\{0\}$ associado às arestas de $G$, e utilizamos a prova construtiva do Teorema 7.7 para obter uma função $s: U \rightarrow \mathbb{R}_{+}$tal que $|\operatorname{supp}(s)| \leq d(n-1)$ e que

$$
1 \leq \lambda_{i}^{\downarrow}\left(\sum_{v \in U} s_{v} v v^{\top}\right) \leq \frac{d+1+2 \sqrt{d}}{d+1-2 \sqrt{d}}
$$

para todo $i \in[n]$. Seja $\vec{G}$ uma orientação de $G$. Defina $W:=\operatorname{Diag}(w)$ e $S:=\operatorname{Diag}(s)$. Pela prova do Teorema 7.9, temos que o grafo com pesos nas arestas $H=\left(V, F, w^{\prime}\right)$, cujo Laplaciano é definido como $L_{H}:=B_{\vec{G}}^{\top} W S B_{\vec{G}}$, é uma $\left(\frac{d+1+2 \sqrt{d}}{d+1-2 \sqrt{d}}\right)$-aproximação de $G$, de forma que o número de arestas de $H$ é no máximo $d(n-1)$. Assim, o esparsificador espectral obtido possui um número linear de arestas em relação ao número de vértices.

Note que, ao limitar os autovalores da matriz formada por sucessivas atualizações de posto 1, estamos, na verdade, limitando os autovalores do esparsificador espectral em progresso. Assim, da mesma forma que podemos obter a matriz formada por sucessivas atualizações de posto 1 de forma construtiva, é possível obter um esparsificador espectral de forma construtiva, limitando os autovalores não nulos do seu Laplaciano a cada iteração.

\subsection{Propriedades expansoras de bons esparsificadores espectrais de grafos completos}

Nesta seção, mostraremos que bons esparsificadores espectrais de grafos completos satisfazem as propriedades de grafos expansores mencionadas no Capítulo 3. Assim, apesar de não serem regulares e possuírem pesos nas arestas, podemos considerá-los bons expansores. 
Como lidamos com grafos com pesos nas arestas, definiremos novamente alguns conceitos. Seja

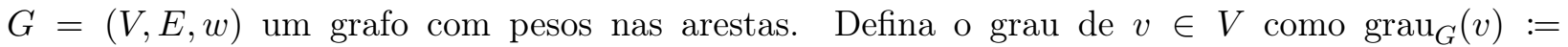

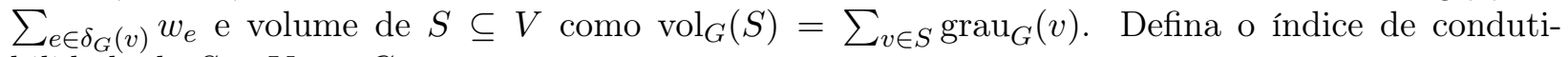
bilidade de $S \subseteq V$ em $G$ como

$$
\phi_{G}(S):=\frac{w_{G}(S)}{\min \left\{\operatorname{vol}_{G}(S), \operatorname{vol}_{G}(\bar{S})\right\}},
$$

e o índice de condutibilidade de $G$ como $\phi(G):=\min _{\varnothing \neq S \subsetneq V} \phi_{G}(S)$. Defina também o peso do corte de $S, T \subseteq V$ em $G$ como

$$
w_{G}(S, T):=\sum_{(u, v) \in E(S, T)} w(\{u, v\}) .
$$

Primeiramente, mostraremos que bons esparsificadores espectrais de grafos completos possuem alta condutibilidade, assim como grafos expansores. Dessa forma, se um conjunto de pontos é representado por vértices de um bom esparsificador espectral de forma que o peso de cada aresta do esparsificador representa a semelhança entre as suas pontas, então esse conjunto de pontos forma um único cluster.

Lema 7.10. Sejam $G$ um grafo e $H=(V, E)$ um grafo que é uma $(1+\varepsilon)$-aproximação $G$. Então

$$
\phi(H) \geq \frac{1}{1+\varepsilon} \phi(G)
$$

Demonstração. Por definição, temos que $L_{G} \preceq L_{H} \preceq(1+\varepsilon) L_{G}$. Logo, para todo $S \subseteq V$ vale que

$$
\mathbb{1}_{S}^{\top} L_{G} \mathbb{1}_{S} \leq \mathbb{1}_{S}^{\top} L_{H} \mathbb{1}_{S} \leq(1+\varepsilon) \mathbb{1}_{S}^{\top} L_{G} \mathbb{1}_{S},
$$

o que implica que $w_{G}(S) \leq w_{H}(S) \leq(1+\varepsilon) w_{G}(S)$. Assim, para todo vértice $v \in V$, vale que $\operatorname{grau}_{G}(v) \leq \operatorname{grau}_{H}(v) \leq(1+\varepsilon) \operatorname{grau}_{G}(v)$, o que implica que

$$
\operatorname{vol}_{G}(S) \leq \operatorname{vol}_{H}(S) \leq(1+\varepsilon) \operatorname{vol}_{G}(S)
$$

para todo $S \subseteq V$. Logo, para todo $S \subseteq V$, vale que

$$
\begin{aligned}
\phi_{H}(S) & =\frac{w_{H}(S)}{\min \left\{\operatorname{vol}_{H}(S), \operatorname{vol}_{H}(\bar{S})\right\}} \\
& \geq \frac{w_{G}(S)}{\min \left\{(1+\varepsilon) \operatorname{vol}_{G}(S),(1+\varepsilon) \operatorname{vol}_{G}(\bar{S})\right\}} \\
& =\frac{w_{G}(S)}{(1+\varepsilon) \min \left\{\operatorname{vol}_{G}(S), \operatorname{vol}_{G}(\bar{S})\right\}} \\
& =\frac{1}{1+\varepsilon} \phi_{G}(S) .
\end{aligned}
$$

Portanto, vale que

$$
\phi(H) \geq \frac{1}{1+\varepsilon} \min _{\varnothing \neq S \subsetneq V} \phi_{G}(S)=\frac{1}{1+\varepsilon} \phi(G) .
$$

Em particular, se $H$ é um bom esparsificador espectral do grafo completo $K_{n}$, então o índice de condutibilidade de $H$ é próximo do índice de condutibilidade de $K_{n}$. Assim, como mostrado no Capítulo 3, grafos completos possuem alta condutibilidade, e portanto, bons esparsificadores espectrais de grafos completos também possuem alta condutibilidade. 
Além dessa característica, bons esparsificadores espectrais de grafos completos também se comportam como grafos aleatórios da mesma forma que grafos expansores: o peso de cada corte de um esparsificador espectral é próximo do peso de seu respectivo corte em um grafo aleatório com o mesmo número de vértices e a mesma densidade.

Lema 7.11 (Expander Mixing Lemma para esparsificadores espectrais). Seja $d \in \mathbb{R}_{++}$e seja $H=$ $(V, E, w)$ um grafo com pesos nas arestas que $(1+\varepsilon)$-aproxima o grafo completo com pesos nas arestas $K_{n}=\left(V,\left(\begin{array}{c}V \\ 2\end{array}\right), \frac{d}{n} \mathbb{1}\right)$. Então, para quaisquer $S, T \subseteq V$ disjuntos,

$$
\left|w_{H}(S, T)-\frac{d|S||T|}{n}\right| \leq d \varepsilon \sqrt{|S||T|}
$$

Demonstração. Denote $K:=K_{n}$. Sejam $S, T \subseteq V$ disjuntos. Por definição, temos que $L_{K} \preceq L_{H} \preceq$ $(1+\varepsilon) L_{K}$. Assim, vale que

$$
0 \preceq L_{H}-L_{K} \preceq \varepsilon L_{K} \preceq \varepsilon d I .
$$

Como $S$ e $T$ são disjuntos, então

$$
\mathbb{1}_{S}^{\top} L_{K} \mathbb{1}_{T}=-w_{K}(S, T)=-\frac{d|S||T|}{n} \quad \text { e } \quad \mathbb{1}_{S}^{\top} L_{H} \mathbb{1}_{T}=-w_{H}(S, T) .
$$

Assim, vale que

$$
\begin{aligned}
-w_{H}(S, T) & =\mathbb{1}_{S}^{\top} L_{H} \mathbb{1}_{T} \\
& =\mathbb{1}_{S}^{\top}\left(L_{K}+\left(L_{H}-L_{K}\right)\right) \mathbb{1}_{T} \\
& =\mathbb{1}_{S}^{\top} L_{K} \mathbb{1}_{T}+\mathbb{1}_{S}^{\top}\left(L_{H}-L_{K}\right) \mathbb{1}_{T} \\
& =-\frac{d|S||T|}{n}+\mathbb{1}_{S}^{\top}\left(L_{H}-L_{K}\right) \mathbb{1}_{T} .
\end{aligned}
$$

Logo, por (7.11), temos que

$$
\begin{array}{rlr}
\left|w_{H}(S, T)-\frac{d|S||T|}{n}\right| & =\left|\mathbb{1}_{S}^{\top}\left(L_{H}-L_{K}\right) \mathbb{1}_{T}\right| & \\
& \leq\left\|\mathbb{1}_{S}\right\|\left\|\left(L_{H}-L_{K}\right) \mathbb{1}_{T}\right\| & \text { por Cauchy-Schwarz } \\
& =\left\|\mathbb{1}_{S}\right\| \sqrt{\mathbb{1}_{T}^{\top}\left(L_{H}-L_{K}\right)^{2} \mathbb{1}_{T}} \\
& =\left\|\mathbb{1}_{S}\right\|\left\|\mathbb{1}_{T}\right\| \sqrt{\frac{\mathbb{1}_{T}^{\top}\left(L_{H}-L_{K}\right)^{2} \mathbb{1}_{T}}{\mathbb{1}_{T}^{\top} \mathbb{1}_{T}}} \\
& \leq\left\|\mathbb{1}_{S}\right\|\left\|\mathbb{1}_{T}\right\| \sqrt{\lambda_{1}^{\downarrow}\left(\left(L_{H}-L_{K}\right)^{2}\right)}
\end{array}
$$

Portanto, como $L_{H}-L_{K} \succeq 0$, então

$$
\begin{aligned}
\left|w_{H}(S, T)-\frac{d|S||T|}{n}\right| & \leq \lambda_{1}^{\downarrow}\left(L_{H}-L_{K}\right)\left\|\mathbb{1}_{S}\right\|\left\|\mathbb{1}_{T}\right\| \\
& \leq d \varepsilon \sqrt{|S||T|}
\end{aligned}
$$$$
\text { por (7.11). }
$$

Considerando a definição de graus de grafos com pesos nas arestas, temos que os graus dos vértices de $(1+\varepsilon)$-aproximações de grafos completos $K_{n}=\left(V,\left(\begin{array}{c}V \\ 2\end{array}\right), \mathbb{1}\right)$, com $\varepsilon$ próximo de 0 , são próximos de $n-1$. Assim, ao multiplicar todos os pesos das arestas de um bom esparsificador espectral $H$ de $K_{n}$ por $d /(n-1)$, os graus dos vértices de $H$ são próximos de $d$. Dessa forma, podemos 
dizer que esses grafos são semelhantes a grafos $d$-regulares. Seja $G(n, p)$ um grafo aleatório no modelo de Erdôs-Rényi com $p=d /(n-1)$ (mesma densidade de $H$ ) e sejam $S, T$ subconjuntos de vértices do grafo aleatório $G(n, p)$. Note que o peso esperado do corte $S, T$ em $G(n, p)$ é $p|S||T|$. Pelo Lema 7.11, ao multiplicar o peso das arestas de $H$ por $p$, o peso de cada corte em $H$ é próximo do peso esperado do respectivo corte do grafo aleatório $G(n, p)$.

Note que, se multiplicarmos os pesos de todas as arestas de um grafo por uma constante, o índice de condutibilidade desse grafo permanece o mesmo. Assim, o resultado do Lema 7.10 se mantém para esparsificadores espectrais de grafos completos com pesos nas arestas multiplicados por $d /(n-1)$. 


\section{Capítulo 8}

\section{Problema de Kadison-Singer}

O Problema de Kadison-Singer [19] é um problema de análise funcional que esteve em aberto desde 1959, e só foi resolvido em 2013 por Marcus, Spielman e Srivastava [25], que provaram conjecturas que implicam uma resposta desse problema.

Enunciaremos o Problema de Kadison-Singer a seguir apenas para que o leitor tenha conhecimento do formato do problema original. Os termos nele utilizados não serão definidos neste texto.

Problema 8.1 (Problema de Kadison-Singer [19]). Todos os estados puros da álgebra de von Neumann $\mathbb{D}$ de um operador diagonal limitado em $\ell_{2}$ possuem uma única extensão para um estado puro na álgebra $B\left(\ell_{2}\right)$ de von Neumann de todos os operadores limitados em $\ell_{2}$ ?

O Problema de Kadison-Singer era de grande interesse para físicos e matemáticos que, com o objetivo de encontrar uma resposta para o problema, criaram muitas conjecturas que acabaram removendo gradualmente suas dificuldades, transformando-o de um problema de análise funcional em um problema de álgebra de matrizes finitas (vide [15, Seção 2]). Muitas dessas conjecturas, que não tinham sido provadas até 2013, são equivalentes à resposta positiva do problema. Assim, ao provar duas conjecturas que implicam, independentemente, a resposta positiva do Problema de Kadison-Singer, Marcus, Spielman e Srivastava não só resolvem o problema que esteve em aberto por mais de 50 anos, como também acabam provando muitas outras conjecturas que estavam, até então, em aberto.

As duas conjecturas provadas em [25] são as conjecturas de Weaver [30] e de Anderson [6]. Neste texto, mostraremos apenas a prova da conjectura de Weaver, enunciada abaixo.

Conjectura (Weaver [30]). Existem reais $\gamma>2$ e $\theta>0$ tais que, para todo inteiro $m \geq 2$ e todos $u_{1}, \ldots, u_{m} \in \mathbb{C}^{n}$ com $\left\|u_{i}\right\|_{2} \leq 1$ para todo $i \in[m]$, vale que, se

$$
\sum_{i \in[m]} u_{i} u_{i}^{*}=\gamma I
$$

então existe uma partição $\left\{S_{1}, S_{2}\right\}$ de $[m]$ tal que $\sum_{i \in S_{j}} u_{i} u_{i}^{*} \preceq(\gamma-\theta) I$ para todo $j \in[2]$.

Tanto a conjectura de Weaver quanto a de Anderson foram demonstradas a partir de um resultado intermediário cuja prova utiliza algumas técnicas usadas na construção de bons expansores que foram apresentadas anteriormente. Dentre elas estão a técnica de limitar as raízes de um polinômio utilizando árvores entrelaçadoras de polinômios e a técnica de controlar raízes de polinômios com barreiras utilizando funções potenciais.

Segundo esse resultado intermediário, apresentado no Teorema 8.13, dados $\varepsilon>0$ e vetores aleatórios $v_{1}, \ldots, v_{m} \in \mathbb{C}^{n}$ de suporte finito cujas normas esperadas são limitadas superiormente por $\varepsilon$, 
existe uma matriz em um conjunto de matrizes positivas semidefinidas $M_{v_{1}, \ldots, v_{m}}$ cujo maior autovalor é limitado por $(1+\sqrt{\varepsilon})^{2}$. Para chegar nesse resultado, mostraremos que a família de polinômios característicos de matrizes pertencentes ao conjunto $M_{v_{1}, \ldots, v_{m}}$ forma uma árvore entrelaçadora de polinômios. Uma propriedade de árvores entrelaçadoras mostrada anteriormente garante que existe uma matriz $A \in M_{v_{1}, \ldots, v_{m}}$ tal que

$$
r_{\max }\left(p_{A}(x)\right) \leq r_{\max }\left(\sum_{A^{\prime} \in M_{v_{1}, \ldots, v_{m}}}\left(p_{A^{\prime}}(x)\right)\right) .
$$

Além disso, construiremos o polinômio $\sum_{A^{\prime} \in M_{v_{1}, \ldots, v_{m}}}\left(p_{A^{\prime}}(x)\right)$ iterativamente com auxílio de barreiras e funções potenciais de forma que seja possível mostrar que $(1+\sqrt{\varepsilon})^{2}$ limita superiormente todas as suas raízes. Dessa forma, limitamos os autovalores de pelo menos uma das matrizes em $M_{v_{1}, \ldots, v_{m}}$.

Note que a técnica utilizada para controlar raízes de polinômios mencionada acima é a mesma usada para provar a existência de bons esparsificadores na seção anterior. Além disso, a técnica de utilizar árvores entrelaçadoras de polinômios para limitar raízes de polinômios também foi utilizada no Capítulo 6 para provar a existência de famílias infinitas de grafos de Ramanujan. Assim, podemos dizer que a prova do Teorema 8.13 é uma combinação das técnicas que foram usadas nos resultados aqui apresentados para construir bons expansores.

\subsection{Mixed Characteristic Polynomial}

Definimos o Mixed Characteristic Polynomial no Capítulo 5 como um polinômio em $\mathbb{C}[x]$ associado a famílias de matrizes quadradas, e mostramos algumas de suas propriedades. Nesta seção, apresentaremos duas formas de representar o Mixed Characteristic Polynomial de famílias de matrizes que obedecem certas restrições. Essas representações serão utilizadas para facilitar a prova de uma conjectura equivalente à resposta positiva para o problema de Kadison-Singer. Segue uma representação do Mixed Characteristic Polynomial de matrizes positivas semidefinidas $A_{1}, \ldots, A_{m}$ que somam para a identidade:

$$
\mu\left[A_{1}, \ldots, A_{m}\right](x)=E_{y=x \mathbb{1}}\left(\left(\prod_{i \in[m]}\left(1-\partial_{y_{i}}\right)\right) \operatorname{det}\left(\sum_{i \in[m]} y_{i} A_{i}\right)\right) .
$$

Utilizamos essa representação (provada no Teorema 8.2) para mostrar que as raízes do Mixed Characteristic Polynomial de matrizes positivas semidefinidas que somam para a identidade são limitadas superiormente por certo valor. Apesar de sua estrutura parecer mais complexa, o lado direito da equação acima representa um polinômio cujo formato nos permite limitar suas raízes utilizando uma função potencial e barreiras, semelhantes às usadas no Capítulo 7.

Sejam $v_{1}, \ldots, v_{m}$ variáveis aleatórias independentes de suporte finito que assumem valores em $\mathbb{C}^{n}$. Como mostrado no Teorema 5.32, a família de polinômios $\left(q_{v_{1}, \ldots, v_{m}}[w]\right)_{w \in R_{v_{1}} \times \cdots \times R_{v_{m}}}$ forma uma árvore entrelaçadora. Além disso, é fácil ver que a soma de todos os polinômios dessa família é o valor esperado do polinômio característico de $\sum_{i \in[m]} v_{i} v_{i}^{*}$. Utilizamos a representação do Mixed Characteristic Polynomial mostrada a seguir para apresentar a soma dos polinômios da família $\left(q_{v_{1}, \ldots, v_{m}}[w]\right)_{w \in R_{v_{1}} \times \cdots \times R_{v_{m}}}$ de outra maneira. O Mixed Characteristic Polynomial de matrizes positivas semidefinidas $A_{1}, \ldots, A_{m}$ tais que $A_{i}:=\mathbb{E}_{v_{i}} v_{i} v_{i}^{*}$ para todo $i \in[m]$ pode ser representado da seguinte maneira:

$$
\mu\left[A_{1}, \ldots, A_{m}\right](x)=\mathbb{E}_{v_{1}, \ldots, v_{m}} \operatorname{det}\left(x I-\sum_{i \in[m]} v_{i} v_{i}^{*}\right) .
$$


Essa equivalência de polinômios foi provada no Corolário 5.29 e será utilizada neste capítulo para mostrar que a família de polinômios $\left(q_{v_{1}, \ldots, v_{m}}[w]\right)_{w \in R_{v_{1}} \times \cdots \times R_{v_{m}}}$, que soma para

$$
\mathbb{E}_{v_{1}, \ldots, v_{m}} \operatorname{det}\left(x I-\sum_{i \in[m]} v_{i} v_{i}^{*}\right)
$$

também soma para $\mu\left[\mathbb{E} v_{1} v_{1}^{*}, \ldots, \mathbb{E} v_{m} v_{m}^{*}\right](x)$. Assim, como essa família de polinômios forma uma árvore entrelaçadora, então as raízes de pelo menos um polinômio dessa família são limitadas pela raiz máxima do Mixed Characteristic Polynomial das matrizes $\mathbb{E} v_{1} v_{1}^{*}, \ldots, \mathbb{E} v_{m} v_{m}^{*}$.

Para limitar as raízes de $\mu\left[\mathbb{E} v_{1} v_{1}^{*}, \ldots, \mathbb{E} v_{m} v_{m}^{*}\right](x)$ encontramos, primeiramente, um limitante superior $\alpha \mathbb{1}$ das raízes do polinômio

$$
\left(\prod_{i \in[m]}\left(1-\partial_{y_{i}}\right)\right) \operatorname{det}\left(\sum_{i \in[m]} y_{i} \mathbb{E} v_{i} v_{i}^{*}\right)
$$

utilizando a técnica de limitar raízes de polinômios (coordenada a coordenada) por barreiras e funções potenciais decrescentes. Note que essa é uma técnica semelhante à utilizada no Capítulo 7. Assim, vale que todas as raízes do polinômio

$$
E_{y=x \mathbb{1}}\left(\left(\prod_{i \in[m]}\left(1-\partial_{y_{i}}\right)\right) \operatorname{det}\left(\sum_{i \in[m]} y_{i} \mathbb{E} v_{i} v_{i}^{*}\right)\right)
$$

são limitadas superiormente por $\alpha$. Dessa forma, podemos utilizar a primeira representação do Mixed Characteristic Polynomial apresentada nesta seção, que será provada no teorema a seguir, para mostrar que esse limitante também é um limitante superior das raízes de $\mu\left[\mathbb{E} v_{1} v_{1}^{*}, \ldots, \mathbb{E} v_{m} v_{m}^{*}\right](x)$.

Teorema 8.2. Sejam $A_{1}, \ldots, A_{m} \in \mathbb{C}^{n \times n}$ matrizes positivas semidefinidas tais que $\sum_{i \in[m]} A_{i}=I$. Sejam $x, y_{1}, \ldots, y_{m}$ indeterminadas algebricamente independentes. Então

$$
\mu\left[A_{1}, \ldots, A_{m}\right](x)=E_{y=x \mathbb{1}}\left(\left(\prod_{i \in[m]}\left(1-\partial_{y_{i}}\right)\right) \operatorname{det}\left(\sum_{i \in[m]} y_{i} A_{i}\right)\right) .
$$

Demonstração. Sejam $z_{1}, \ldots, z_{m}$ indeterminadas algebricamente independentes. Pela Proposição 2.9, temos que

$$
\begin{aligned}
\mu\left[A_{1}, \ldots, A_{m}\right](x) & =E_{z_{1}=\cdots=z_{m}=0}\left(\left(\prod_{j \in[m]}\left(1-\partial_{z_{j}}\right)\right) \operatorname{det}\left(x I+\sum_{i \in[m]} z_{i} A_{i}\right)\right) \\
& =E_{y=x \mathbb{1}}\left(E_{z=y-x \mathbb{1}}\left(\left(\prod_{j \in[m]}\left(1-\partial_{z_{j}}\right)\right) \operatorname{det}\left(x I+\sum_{i \in[m]} z_{i} A_{i}\right)\right)\right) .
\end{aligned}
$$

Para todo $k \in\{0, \ldots, m\}$, defina

$$
\begin{gathered}
r_{k}:=\operatorname{det}\left(x I+\sum_{i \in[k]}\left(\left(y_{i}-x\right) A_{i}\right)+\sum_{i=k+1}^{m} z_{i} A_{i}\right), \\
\bar{E}_{k}:=\prod_{i \in[k]}\left(E_{z_{i}=y_{i}-x} \circ\left(1-\partial_{z_{i}}\right)\right) .
\end{gathered}
$$

Provaremos por indução em $k$ que $\bar{E}_{k}\left(r_{0}\right)=\left(\prod_{i \in[k]}\left(1-\partial_{y_{i}}\right)\right) r_{k}$. 
Se $k=0$, é fácil ver que o resultado vale.

Suponha que $k \geq 1$. Pela hipótese de indução, temos que

$$
\bar{E}_{k-1}\left(r_{0}\right)=\left(\prod_{i \in[k-1]}\left(1-\partial_{y_{i}}\right)\right)\left(r_{k-1}\right) .
$$

Note que $\bar{E}_{k}\left(r_{0}\right)=\left(E_{z_{k}=y_{k}-x} \circ\left(1-\partial_{z_{k}}\right)\right)\left(\bar{E}_{k-1}\left(r_{0}\right)\right)$. Dessa forma, vale que

$$
\begin{aligned}
\bar{E}_{k}\left(r_{0}\right) & =\left(E_{z_{k}=y_{k}-x} \circ\left(1-\partial_{z_{k}}\right)\right)\left(\left(\prod_{i \in[k-1]}\left(1-\partial_{y_{i}}\right)\right)\left(r_{k-1}\right)\right) \\
& =\left(\prod_{i \in[k-1]}\left(1-\partial_{y_{i}}\right)\right)\left(\left(E_{z_{k}=y_{k}-x} \circ\left(1-\partial_{z_{k}}\right)\right)\left(r_{k-1}\right)\right) \\
& =\left(\prod_{i \in[k-1]}\left(1-\partial_{y_{i}}\right)\right)\left(E_{z_{k}=y_{k}-x}\left(r_{k-1}-\partial_{z_{k}} r_{k-1}\right)\right) .
\end{aligned}
$$

Pelas Proposições 2.22 e 2.8, temos que

$$
\begin{aligned}
E_{z_{k}=y_{k}-x}\left(r_{k-1}-\partial_{z_{k}} r_{k-1}\right) & =E_{z_{k}=y_{k}-x}\left(r_{k-1}\right)-E_{z_{k}=y_{k}-x}\left(\partial_{z_{k}} r_{k-1}\right) \\
& =r_{k}-E_{z_{k}=y_{k}-x}\left(\partial_{z_{k}} r_{k-1}\right) \\
& =r_{k}-E_{z_{k}=y_{k}-x} \circ \partial_{z_{k}}\left(\operatorname{det}\left(x I+\sum_{i=1}^{k-1}\left(\left(y_{i}-x\right) A_{i}\right)+\sum_{i=k}^{m} z_{i} A_{i}\right)\right) \\
& =r_{k}-\partial_{y_{k}}\left(\operatorname{det}\left(x I+\sum_{i=1}^{k}\left(\left(y_{i}-x\right) A_{i}\right)+\sum_{i=k+1}^{m} z_{i} A_{i}\right)\right) \\
& =r_{k}-\partial_{y_{k}} r_{k} \\
& =\left(1-\partial_{y_{k}}\right) r_{k} .
\end{aligned}
$$

Logo, por (8.2), temos que

$$
\bar{E}_{k}\left(r_{0}\right)=\left(\prod_{i \in[k-1]}\left(1-\partial_{y_{i}}\right)\right)\left(\left(1-\partial_{y_{k}}\right) r_{k}\right) .
$$

Portanto, vale que $\bar{E}_{k}\left(r_{0}\right)=\left(\prod_{i \in[k]}\left(1-\partial_{y_{i}}\right)\right) r_{k}$ para todo $k \in\{0, \ldots, m\}$.

Pela Proposição 2.12, vale que

$$
\bar{E}_{m}\left(r_{0}\right)=E_{z=y-x \mathbb{1}}\left(\left(\prod_{j \in[m]}\left(1-\partial_{z_{j}}\right)\right) \operatorname{det}\left(x I+\sum_{i \in[m]} z_{i} A_{i}\right)\right) .
$$

Logo, por (8.1), temos que

$$
\begin{aligned}
E_{z_{1}=\cdots=z_{m}=0} & \left(\left(\prod_{j \in[m]}\left(1-\partial_{z_{j}}\right)\right) \operatorname{det}\left(x I+\sum_{i \in[m]} z_{i} A_{i}\right)\right) \\
& =E_{y=x \mathbb{1}}\left(\bar{E}_{m}\left(r_{0}\right)\right) \\
& =E_{y=x \mathbb{1}}\left(\left(\prod_{i \in[m]}\left(1-\partial_{y_{i}}\right)\right) r_{m}\right)
\end{aligned}
$$




$$
\begin{aligned}
& =E_{y=x \mathbb{1}}\left(\left(\prod_{i \in[m]}\left(1-\partial_{y_{i}}\right)\right) \operatorname{det}\left(x I+\sum_{i=1}^{m}\left(\left(y_{i}-x\right) A_{i}\right)\right)\right) \\
& =E_{y=x \mathbb{1}}\left(\left(\prod_{i \in[m]}\left(1-\partial_{y_{i}}\right)\right) \operatorname{det}\left(\sum_{i=1}^{m} y_{i} A_{i}\right)\right),
\end{aligned}
$$

o que implica que

$$
\mu\left[A_{1}, \ldots, A_{m}\right](x)=E_{y=x \mathbb{1}}\left(\left(\prod_{i \in[m]}\left(1-\partial_{y_{i}}\right)\right) \operatorname{det}\left(\sum_{i \in[m]} y_{i} A_{i}\right)\right) .
$$

Utilizaremos as propriedades do Mixed Characteristic Polynomial apresentadas anteriormente, juntamente com propriedades de árvores entrelaçadoras de polinômios e função potencial (que será definida na próxima seção), para mostrar um resultado intermediário que implica em uma conjectura equivalente à resposta positiva do Problema de Kadison-Singer. Segundo esse resultado intermediário, a probabilidade de certa variável aleatória assumir o valor de um polinômio cujas raízes são limitadas por certo valor é não nula.

\subsection{Barreiras e função potencial}

No Capítulo 7, utilizamos funções potenciais (inferior e superior) e barreiras para construir iterativamente grafos cujos autovalores de seu Laplaciano em progresso são limitados inferior e superiormente pelas barreiras referentes à iteração em questão. Neste capítulo, utilizamos uma função potencial superior e barreiras para construir iterativamente o Mixed Characteristic Polynomial, de matrizes positivas semidefinidas cuja soma é a identidade, de forma que a função potencial garanta que suas raízes sejam limitadas superiormente pelas barreiras.

A função potencial tem o mesmo papel das funções potenciais utilizadas para limitar os autovalores de esparsificadores espectrais. Elas quantificam a proximidade entre as raízes do polinômio em questão e uma barreira. Além disso, se o potencial não aumenta a cada iteração, então as raízes desse polinômio permanecem limitadas superiormente pela barreira. Porém, apesar de também termos como objetivo limitar as raízes de polinômios monovariados neste capítulo, utilizamos uma função potencial de polinômios multivariados, pois o Mixed Characteristic Polynomial pode ser obtido através de um polinômio multivariado, como mostrado no Teorema 8.2.

Sejam $A_{1}, \ldots, A_{m}$ matrizes complexas positivas semidefinidas cuja soma é a identidade. Para mostrar que as raízes do polinômio $\mu\left[A_{1}, \ldots, A_{m}\right](x)$ são limitadas superiormente por certo valor, construímos esse polinômio iterativamente a partir de um polinômio multivariado com indeterminadas $y_{1}, \ldots, y_{m}$ (vide Teorema 8.2). O Mixed Characteristic Polynomial é obtido através de sucessivas operações $\left(1-\partial_{y_{i}}\right)$, para todo $i \in[m]$, sobre o polinômio multivariado, seguidas pela especificação de todas as indeterminadas do polinômio resultante para uma única indeterminada $x$.

Como estamos lidando com polinômios multivariados, definiremos limitantes superiores de raízes de polinômios multivariados e uma função potencial para barreiras multivariadas.

Definição 8.3 (Limitante superior de raízes). Seja $p \in \mathbb{R}\left[x_{1}, \ldots, x_{m}\right]$. Dizemos que $u \in \mathbb{R}^{m}$ é um limitante superior das raizes de $p$ se $p(u+y)>0$ para todo $y \in \mathbb{R}_{+}^{m}$.

Note que um limitante superior das raízes de um polinômio $p$ limita superiormente todas as raízes reais de $p$ em pelo menos uma coordenada. Por exemplo, se $u \in \mathbb{R}^{m}$ é um limitante superior das raízes de $p$, então, para toda raiz $x \in \mathbb{R}^{m}$ de $p$, pelo menos uma das inequações abaixo vale:

$$
x_{1}<u_{1} \quad \text { ou } \quad x_{2}<u_{2} \quad \text { ou } \quad \cdots \quad \text { ou } \quad x_{m}<u_{m} .
$$




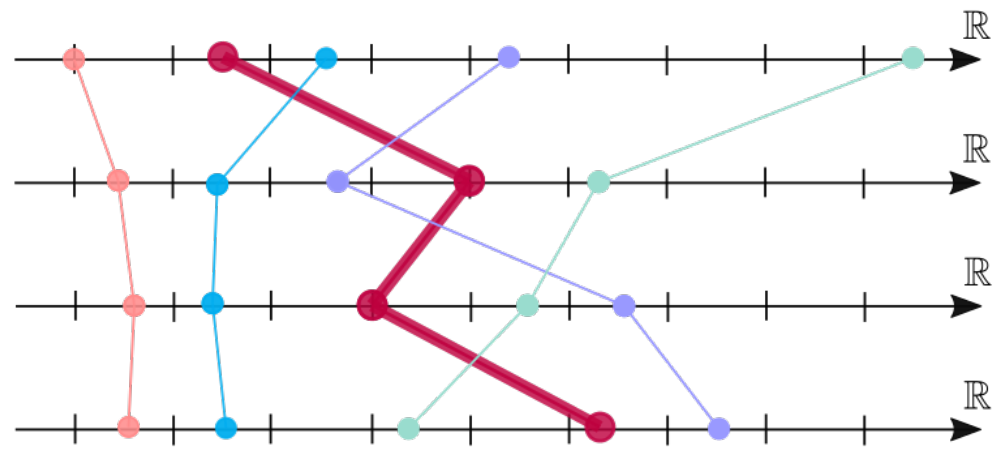

- limitante das raízes de $p$

$\equiv$ raízes de $p$

Figura 8.1: Representação de um limitante superior das raízes de um polinômio $p$, onde cada eixo representa uma dimensão.

Seja $p \in \mathbb{R}\left[x_{1}, \ldots, x_{m}\right]$ um polinômio com $m>1$ e seja $u$ um limitante superior das raízes de $p$. Sejam $\alpha_{1}, \ldots, \alpha_{m-1} \in \mathbb{R}$ com $\alpha_{i} \geq u_{i}$ para todo $i \in[m-1]$. Ao especificar as indeterminadas $x_{1}, \ldots, x_{m-1}$ de $p$ por $\alpha_{1}, \ldots, \alpha_{m-1}$, respectivamente, obtemos um polinômio monovariado $\bar{p} \in \mathbb{R}\left[x_{m}\right]$. Seja $\alpha_{m} \in \mathbb{R}$ uma raiz de $\bar{p}$. Note que $\left(\alpha_{1}, \ldots, \alpha_{m-1}, \alpha_{m}\right)$ é uma raiz real de $p$. Assim, como $u$ é um limitante superior das raízes de $p$ e $\alpha_{i} \geq u_{i}$ para todo $i \in[m-1]$, então $\alpha_{m}<u_{m}$. Logo, $u$ limita a raiz $\left(\alpha_{1}, \ldots, \alpha_{m}\right)$ de $p$ em apenas uma coordenada. Por esse motivo, o limitante superior de raízes de polinômios multivariados foi definido de forma a limitar o valor de pelo menos uma das coordenadas de todas as raízes do polinômio em questão.

Definição 8.4 (Função potencial). Sejam $p \in \mathbb{R}\left[x_{1}, \ldots, x_{m}\right]$ um polinômio real estável e $i \in[m]$. Defina a função potencial $\Phi_{p}^{i} \in \mathbb{R}\left(x_{1}, \ldots, x_{m}\right)$ do polinômio $p$ na posição $i$ como

$$
\Phi_{p}^{i}(x):=\frac{\partial_{x_{i}} p(x)}{p(x)} .
$$

Definição 8.5. Sejam $p \in \mathbb{R}\left[x_{1}, \ldots, x_{m}\right]$ um polinômio real estável, $i \in[m]$ e $u \in \mathbb{R}^{m}$ um limitante superior das raízes de $p$. Seja $f:\left\{x_{1}, \ldots, x_{m}\right\} \backslash\left\{x_{i}\right\} \rightarrow \mathbb{R}$ definida como $f\left(x_{j}\right)=u_{j}$ para todo $j \in[m] \backslash\{i\}$. Defina $\phi_{p, u}^{i} \in \mathbb{R}\left[x_{i}\right]$ como

$$
\phi_{p, u}^{i}\left(x_{i}\right):=E_{f}\left(p\left(x_{1}, \ldots, x_{m}\right)\right) .
$$

Note que $\phi_{p, u}^{i}$ é um polinômio monovariado formado pelos valores de $u$ que não depende do valor da $i$-ésima coordenada de $u$. Assim, se $v$ e $w$ são limitantes superiores das raízes de $p$ com $v_{j}=w_{j}$ para todo $j \in[m] \backslash\{i\}$, então $\phi_{p, v}^{i}=\phi_{p, w}^{i}$.

Proposição 8.6. Sejam $p \in \mathbb{R}\left[x_{1}, \ldots, x_{m}\right]$ um polinômio real estável, $i \in[m]$ e $u \in \mathbb{R}^{m}$ um limitante superior das raízes de $p$. Seja $d$ o grau de $\phi_{p, u}^{i}$. Então

$$
E_{x=u}\left(\Phi_{p}^{i}(x)\right)=\sum_{j \in[d]} \frac{1}{u_{i}-r_{j}\left(\phi_{p, u}^{i}\right)},
$$

onde $r_{1}\left(\phi_{p, u}^{i}\right), \ldots, r_{d}\left(\phi_{p, u}^{i}\right)$ são as raízes de $\phi_{p, u}^{i}$. 
Demonstração. Seja $f:\left\{x_{1}, \ldots, x_{m}\right\} \backslash\left\{x_{i}\right\} \rightarrow \mathbb{R}$ uma função definida como $f\left(x_{j}\right)=u_{j}$ para todo $j \in[m] \backslash\{i\}$. Como $u$ é um limitante superior das raízes de $p$, então $p(u)>0$. Assim, vale que

$$
\begin{array}{rlr}
E_{x=u}\left(\Phi_{p}^{i}(x)\right) & =E_{x=u}\left(\frac{\partial_{x_{i}} p(x)}{p(x)}\right) & \\
& =E_{x_{i}=u_{i}}\left(E_{f}\left(\frac{\partial_{x_{i}} p(x)}{p(x)}\right)\right) & \\
& =E_{x_{i}=u_{i}}\left(\frac{E_{f}\left(\partial_{x_{i}} p(x)\right)}{E_{f}(p(x))}\right) & \text { pela Proposição } 2.8 \\
& =E_{x_{i}=u_{i}}\left(\frac{\partial_{x_{i}} E_{f}(p(x))}{E_{f}(p(x))}\right) & \\
& =E_{x_{i}=u_{i}}\left(\frac{\partial_{x_{i}} \phi_{p, u}^{i}\left(x_{i}\right)}{\phi_{p, u}^{i}\left(x_{i}\right)}\right) . &
\end{array}
$$

Como o polinômio $\phi_{p, u}^{i}\left(x_{i}\right) \in \mathbb{R}\left[x_{i}\right]$ é monovariado, então ele pode ser escrito da seguinte forma:

$$
\phi_{p, u}^{i}\left(x_{i}\right)=\prod_{j \in[d]}\left(x_{i}-r_{j}\left(\phi_{p, u}^{i}\right)\right) .
$$

Logo, vale que

$$
\partial_{x_{i}} \phi_{p, u}^{i}\left(x_{i}\right)=\sum_{j \in[d]}\left(\prod_{\ell \in[d] \backslash\{j\}}\left(x_{i}-r_{\ell}\left(\phi_{p, u}^{i}\right)\right)\right) .
$$

Portanto, por (8.3), temos que

$$
\begin{aligned}
E_{x=u}\left(\Phi_{p}^{i}(x)\right) & =E_{x_{i}=u_{i}}\left(\frac{\sum_{j \in[d]}\left(\prod_{\ell \in[d] \backslash\{j\}}\left(x_{i}-r_{\ell}\left(\phi_{p, u}^{i}\right)\right)\right)}{\prod_{j \in[d]}\left(x_{i}-r_{j}\left(\phi_{p, u}^{i}\right)\right)}\right) \\
& =E_{x_{i}=u_{i}}\left(\sum_{j \in[d]} \frac{1}{x_{i}-r_{j}\left(\phi_{p, u}^{i}\right)}\right) \\
& =\sum_{j \in[d]} \frac{1}{u_{i}-r_{j}\left(\phi_{p, u}^{i}\right)}
\end{aligned}
$$

pela Proposição 2.8 .

Apesar de $\Phi_{p}^{i}(x)$ ser um elemento de $\mathbb{R}\left(x_{1}, \ldots, x_{m}\right)$, utilizamos, no resultado anterior, propriedades de polinômios reais que também valem para $\mathbb{R}\left(x_{1}, \ldots, x_{m}\right)$ caso o divisor seja diferente de 0 . Sejam $p \in \mathbb{R}\left[x_{1}, \ldots, x_{m}\right]$ e $u \in \mathbb{R}^{m}$ um limitante superior das raízes de $p$, e seja $i \in[m]$. Denotamos $E_{x=u}\left(\Phi_{p}^{i}(x)\right)$ por potencial de $u$ na coordenada $i$ em relação ao polinômio $p$, ou somente $\Phi_{p}^{i}$ de $u$.

O potencial $\Phi_{p}^{i}$ de um limitante superior $u$ das raízes de $p$ quantifica a proximidade entre o valor do limitante na $i$-ésima coordenada $u_{i}$ e as raízes do polinômio monovariado que resulta da especificação de todas as indeterminadas de $p$, exceto $x_{i}$, pelos respectivos valores da barreira. Note que a função potencial definida nesta seção é semelhante à função potencial apresentada no capítulo de esparsificadores espectrais. Sejam $u \in \mathbb{R}_{++}$e $x$ uma indeterminada. Seja $A$ uma matriz positiva semidefinida tal que $A \prec u I$. Note que $\Phi^{u}(A)=\Phi_{\operatorname{det}(x I-A)}^{1}(u)$, onde $\Phi^{u}$ é a função potencial superior apresentada na Definição 7.2.

Nesta seção mostraremos que o potencial de um polinômio real estável decresce à medida que a barreira é deslocada para a direita em uma de suas coordenadas. Além disso, se deslocamos a barreira de um polinômio real estável $p$ para a direita em uma de suas coordenadas, o potencial de 
$p$ também decresce ao aplicar a operação $\left(1-\partial_{x_{i}}\right)$ em $p$, onde $x_{i}$ é uma das indeterminadas de $p$. Para mostrar que a função potencial decresce nesses casos, utilizamos algumas de suas propriedades: monotonicidade e convexidade coordenada a coordenada. Essas propriedades serão mostradas nos Lemas 8.8 e 8.9, respectivamente, e suas provas utilizam outra propriedade da função potencial que será apresentada a seguir.

Lema 8.7. Seja $m \geq 2$. Sejam $p \in \mathbb{R}\left[x_{1}, \ldots, x_{m}\right]$ um polinômio real estável e $u \in \mathbb{R}^{m}$ um limitante superior das raízes de $p$. Sejam $i, j \in[m]$ distintos. Então existem matrizes positivas semidefinidas $A_{i}, A_{j} \in \mathbb{C}^{n \times n}$ e uma matriz hermitiana $A \in \mathbb{C}^{n \times n}$, onde $B:=u_{i} A_{i}+u_{j} A_{j}+A$ é positiva definida e

$$
E_{x=u+\varepsilon e_{j}}\left(\partial_{x_{j}} \Phi_{p}^{i}(x)\right)=-\operatorname{Tr}\left(A_{j}\left(\varepsilon A_{j}+B\right)^{-1} A_{i}\left(\varepsilon A_{j}+B\right)^{-1}\right)
$$

para todo $\varepsilon \geq 0$.

Demonstração. Seja $f:\left\{x_{1}, \ldots, x_{m}\right\} \backslash\left\{x_{i}, x_{j}\right\} \rightarrow\left\{u_{1}, \ldots, u_{m}\right\} \backslash\left\{u_{i}, u_{j}\right\}$ uma função definida como $f\left(x_{k}\right)=u_{k}$ para todo $k \in[m] \backslash\{i, j\}$. Defina $\bar{p} \in \mathbb{R}\left[x_{i}, x_{j}\right]$ como

$$
\bar{p}\left(x_{i}, x_{j}\right):=E_{f}(p(x)) .
$$

Pelo Corolário 5.18, temos que existem $A_{i}, A_{j} \in \mathbb{C}^{n \times n}$ positivas semidefinidas e $A \in \mathbb{C}^{n \times n}$ hermitiana tais que

$$
\bar{p}\left(x_{i}, x_{j}\right)= \pm \operatorname{det}\left(x_{i} A_{i}+x_{j} A_{j}+A\right)
$$

e $\alpha_{i} A_{i}+\alpha_{j} A_{j} \succ 0$ para todos $\alpha_{i}, \alpha_{j} \in \mathbb{R}_{++}$. Dessa forma, existem $\alpha_{i}, \alpha_{j} \in \mathbb{R}$ tais que

$$
\beta_{i} A_{i}+\beta_{j} A_{j}+A \succ 0
$$

para todos $\beta_{i}, \beta_{j} \in \mathbb{R} \operatorname{com} \beta_{i} \geq \alpha_{i}$ e $\beta_{j} \geq \alpha_{j}$. Como $u$ é um limitante superior das raízes de $p$, então $\bar{p}\left(u_{i}+\alpha_{i}, u_{j}+\alpha_{j}\right)>0$ para todos $\alpha_{i}, \alpha_{j} \in \mathbb{R}_{+}$. Logo, vale que

$$
\bar{p}\left(x_{i}, x_{j}\right)=\operatorname{det}\left(x_{i} A_{i}+x_{j} A_{j}+A\right) .
$$

Além disso, como $p$ é real estável e $u \in \mathbb{R}^{m}$, então, pelo Corolário 5.7, temos que $\bar{p}$ também é real estável.

Suponha, por contradição, que $B:=u_{i} A_{i}+u_{j} A_{j}+A$ não é uma matriz positiva definida. Como $A_{i}+A_{j} \succ 0$, então existe $\varepsilon>0$ tal que

$$
\varepsilon\left(A_{i}+A_{j}\right)+u_{i} A_{i}+u_{j} A_{j}+A \succeq 0
$$

o que implica que $\lambda_{n}^{\downarrow}\left(\varepsilon\left(A_{i}+A_{j}\right)+u_{i} A_{i}+u_{j} A_{j}+A\right) \geq 0$. Além disso, se $\varepsilon=0$, vale que

$$
\lambda_{n}^{\downarrow}\left(\varepsilon\left(A_{i}+A_{j}\right)+u_{i} A_{i}+u_{j} A_{j}+A\right)=\lambda_{n}^{\downarrow}(B) \leq 0 .
$$

Logo, pelo Lema 4.18 e pelo Teorema do Valor Intermediário, existe $\varepsilon^{\prime} \geq 0$ tal que

$$
\lambda_{n}^{\downarrow}\left(\varepsilon^{\prime}\left(A_{i}+A_{j}\right)+u_{i} A_{i}+u_{j} A_{j}+A\right)=0,
$$

o que implica que

$$
\bar{p}\left(u_{i}+\varepsilon^{\prime}, u_{j}+\varepsilon^{\prime}\right)=\operatorname{det}\left(\left(u_{i}+\varepsilon^{\prime}\right) A_{i}+\left(u_{j}+\varepsilon^{\prime}\right) A_{j}+A\right)=0 .
$$

Como $u+\varepsilon^{\prime} e_{i}+\varepsilon^{\prime} e_{j}$ é um limitante superior das raízes de $p$, então $\bar{p}\left(u_{i}+\varepsilon^{\prime}, u_{j}+\varepsilon^{\prime}\right)>0$, o que nos leva a uma contradição. 
Note que, como $u$ é um limitante superior das raízes de $p$, então $\bar{p}\left(u_{i}, u_{j}\right)>0$. Como, além disso, $B \succ 0$, então $\operatorname{det}(B)>0$.

Pelo Lema 2.21, temos que

$$
\begin{aligned}
\Phi_{p}^{i}(u) & =\frac{E_{x_{i}=u_{i}}\left(\partial_{x_{i}} \bar{p}\left(x_{i}, u_{j}\right)\right)}{\bar{p}\left(u_{i}, u_{j}\right)} \\
& =\frac{E_{x_{i}=u_{i}}\left(\partial_{x_{i}} \operatorname{det}\left(x_{i} A_{i}+u_{j} A_{j}+A\right)\right.}{\operatorname{det}\left(u_{i} A_{i}+u_{j} A_{j}+A\right)} \\
& =\frac{\operatorname{det}\left(u_{i} A_{i}+u_{j} A_{j}+A\right) \operatorname{Tr}\left(\left(u_{i} A_{i}+u_{j} A_{j}+A\right)^{-1} A_{i}\right)}{\operatorname{det}\left(u_{i} A_{i}+u_{j} A_{j}+A\right)} \\
& =\operatorname{Tr}\left(\left(u_{i} A_{i}+u_{j} A_{j}+A\right)^{-1} A_{i}\right) .
\end{aligned}
$$

Logo, vale que $\Phi_{p}^{i}(u)=\operatorname{Tr}\left(B^{-1} A_{i}\right)$. Seja $\delta>0$ suficientemente pequeno para que

$$
\rho\left(\delta A_{j} B^{-1}\right)<1 .
$$

Dessa forma, vale que $I+\delta A_{j} B^{-1}$ é inversível. Assim, vale que

$$
\begin{aligned}
\Phi_{p}^{i}\left(u+\delta e_{j}\right) & =\operatorname{Tr}\left(\left(B+\delta A_{j}\right)^{-1} A_{i}\right) \\
& =\operatorname{Tr}\left(B^{-1}\left(I+\delta A_{j} B^{-1}\right)^{-1} A_{i}\right) \\
& =\operatorname{Tr}\left(\left(I+\delta A_{j} B^{-1}\right)^{-1} A_{i} B^{-1}\right)
\end{aligned}
$$

Pela Proposição 2.4, temos que $\left(I+\delta A_{j} B^{-1}\right)^{-1}=\sum_{k \in \mathbb{N}}\left(-\delta A_{j} B^{-1}\right)^{k}$. Logo, pela Proposição 2.13, temos que

$$
\begin{aligned}
E_{x=u}\left(\partial_{x_{j}} \Phi_{p}^{i}(x)\right) & =\lim _{\delta \rightarrow 0} \frac{\Phi_{p}^{i}\left(u+\delta e_{j}\right)-\Phi_{p}^{i}(u)}{\delta} \\
& =\lim _{\delta \rightarrow 0} \frac{\operatorname{Tr}\left(\left(I+\delta A_{j} B^{-1}\right)^{-1} A_{i} B^{-1}\right)-\operatorname{Tr}\left(A_{i} B^{-1}\right)}{\delta} \\
& =\lim _{\delta \rightarrow 0} \frac{\operatorname{Tr}\left(\sum_{k \in \mathbb{N}}\left(-\delta A_{j} B^{-1}\right)^{k} A_{i} B^{-1}\right)-\operatorname{Tr}\left(A_{i} B^{-1}\right)}{\delta} \\
& =\lim _{\delta \rightarrow 0} \frac{\sum_{k \in \mathbb{N}} \operatorname{Tr}\left(\left(-\delta A_{j} B^{-1}\right)^{k} A_{i} B^{-1}\right)-\operatorname{Tr}\left(A_{i} B^{-1}\right)}{\delta} .
\end{aligned}
$$

Note que

$\sum_{k \in \mathbb{N}} \operatorname{Tr}\left(\left(-\delta A_{j} B^{-1}\right)^{k} A_{i} B^{-1}\right)=\operatorname{Tr}\left(A_{i} B^{-1}\right)+\operatorname{Tr}\left(-\delta A_{j} B^{-1} A_{i} B^{-1}\right)+\sum_{k \in \mathbb{N}} \operatorname{Tr}\left(\left(-\delta A_{j} B^{-1}\right)^{k+2} A_{i} B^{-1}\right)$.

Logo, vale que

$$
\begin{aligned}
E_{x=u}\left(\partial_{x_{j}} \Phi_{p}^{i}(x)\right) & =\lim _{\delta \rightarrow 0} \frac{\operatorname{Tr}\left(-\delta A_{j} B^{-1} A_{i} B^{-1}\right)+\sum_{k \in \mathbb{N}} \operatorname{Tr}\left(\left(-\delta A_{j} B^{-1}\right)^{k+2} A_{i} B^{-1}\right)}{\delta} \\
& =\lim _{\delta \rightarrow 0} \frac{-\delta \operatorname{Tr}\left(A_{j} B^{-1} A_{i} B^{-1}\right)+\delta^{2} \sum_{k \in \mathbb{N}} \delta^{k} \operatorname{Tr}\left(\left(-A_{j} B^{-1}\right)^{k+2} A_{i} B^{-1}\right)}{\delta} \\
& =\lim _{\delta \rightarrow 0}\left(-\operatorname{Tr}\left(A_{j} B^{-1} A_{i} B^{-1}\right)+\delta \sum_{k \in \mathbb{N}} \delta^{k} \operatorname{Tr}\left(\left(-A_{j} B^{-1}\right)^{k+2} A_{i} B^{-1}\right)\right) \\
& =-\operatorname{Tr}\left(A_{j} B^{-1} A_{i} B^{-1}\right) .
\end{aligned}
$$


Note que, para todo $\varepsilon>0$, vale que $\Phi_{p}^{i}\left(u+\varepsilon e_{j}\right)=\frac{\partial_{u_{i}} \bar{p}\left(u_{i}, u_{j}+\varepsilon\right)}{\bar{p}\left(u_{i}, u_{j}+\varepsilon\right)}$. Além disso, $u+\varepsilon e_{j}$ também é um limitante superior das raízes de $p$, o que implica que

$$
E_{x=u+\varepsilon e_{j}}\left(\partial_{x_{j}} \Phi_{p}^{i}(x)\right)=-\operatorname{Tr}\left(A_{j}\left(\varepsilon A_{j}+B\right)^{-1} A_{i}\left(\varepsilon A_{j}+B\right)^{-1}\right) .
$$

Utilizamos o resultado apresentado acima para mostrar que o potencial de polinômios reais estáveis se comporta como uma função decrescente e convexa coordenada a coordenada. Mostraremos, primeiramente, a monotonicidade da função potencial. Seja $p \in \mathbb{R}\left[x_{1}, \ldots, x_{m}\right]$ um polinômio real estável, $u \in \mathbb{R}^{m}$ um limitante superior das raízes de $p$ e $j \in[m]$. Mostraremos que, ao especificar $m-1$ indeterminadas $x_{i}$ de $p$ para $u_{i}$ para todo $i \in[m] \operatorname{com} i \neq j$, o potencial do polinômio monovariado que resulta dessa especificação é decrescente em $\left[u_{j},+\infty\right)$. Assim, vale que $\Phi_{p}^{i}(u) \geq \Phi_{p}^{i}\left(u+\delta e_{j}\right)$ para todo $\delta>0$ e todo $i \in[m]$. Em outras palavras, o potencial de $p$ associado a qualquer coordenada diminui à medida que o valor de uma coordenada de um limitante superior das raízes de $p$ é aumentado. Além disso, é fácil ver que o potencial de $p$ associado a qualquer coordenada também diminui quando é acrescido um vetor com valores positivos ao limitante.

Lema 8.8 (Marcus, Spielman e Srivastava [25]). Sejam $p \in \mathbb{R}\left[x_{1}, \ldots, x_{m}\right]$ um polinômio real estável e $u \in \mathbb{R}^{m}$ um limitante superior das raízes de $p$. Sejam $i, j \in[m]$. Então

$$
E_{x=u}\left(\partial_{x_{j}} \Phi_{p}^{i}(x)\right) \leq 0 .
$$

Demonstração. Seja $d$ o grau de $\phi_{p, u}^{i}$ e seja $\delta>0$. Como $u$ é um limitante superior das raízes de $p$ e $u+\delta e_{j} \geq u$, então $u+\delta e_{j}$ também é um limitante superior das raízes de $p$.

Se $i=j$, então $\phi_{p, u+\delta e_{j}}^{i}=\phi_{p, u+\delta e_{i}}^{i}=\phi_{p, u}^{i}$. Como $u$ é um limitante superior das raízes de $p$, então $u_{i}>r_{k}\left(\phi_{p, u}^{i}\right)$, para todo $k \in[d]$, o que implica que

$$
u_{i}+\delta-r_{k}\left(\phi_{p, u}^{i}\right)>u_{i}-r_{k}\left(\phi_{p, u}^{i}\right)>0 .
$$

Assim, pela Proposição 8.6, temos que $\Phi_{p}^{i}(u)>\Phi_{p}^{i}\left(u+\delta e_{i}\right)$.

Suponha agora que $i \neq j$. Pelo Lema 8.7, temos que existem matrizes positivas semidefinidas $A_{i}, A_{j} \in \mathbb{C}^{n \times n}$ e uma matriz hermitiana $A \in \mathbb{C}^{n \times n}$, onde $B:=u_{i} A_{i}+u_{j} A_{j}+A$ é positiva definida e

$$
E_{x=u}\left(\partial_{x_{j}} \Phi_{p}^{i}(x)\right)=-\operatorname{Tr}\left(A_{j} B^{-1} A_{i} B^{-1}\right) .
$$

Como $A_{i}$ é positiva semidefinida, então $B^{-1} A_{i} B^{-1}$ também é positiva semidefinida. Portanto, pela Proposição 2.43, temos que $E_{x=u}\left(\partial_{x_{j}} \Phi_{p}^{i}(x)\right) \leq 0$.

A segunda propriedade da função potencial mencionada anteriormente é a convexidade coordenada a coordenada. Em outras palavras, a função potencial de um polinômio se comporta como uma função convexa ao fixar valores para todas as indeterminadas do polinômio em questão, exceto uma. A convexidade segue a mesma ideia da monotonicidade coordenada a coordenada, onde a função se comporta de forma monótona quando fixamos os valores de todas as indeterminadas do polinômio, exceto uma. Seja $p$ um polinômio real estável, $u \in \mathbb{R}^{m}$ um limitante superior das raízes de $p$ e $j \in[m]$. Defina $\bar{p}$ como o polinômio monovariado resultante da especificação de $m-1$ indeterminadas distintas $x_{i} \mapsto u_{i}$ no polinômio $p$. Mostraremos que a função potencial de $\bar{p}$ é convexa em $\left[u_{j},+\infty\right)$.

Lema 8.9 (Marcus, Spielman e Srivastava [25]). Sejam $p \in \mathbb{R}\left[x_{1}, \ldots, x_{m}\right]$ um polinômio real estável e $u \in \mathbb{R}^{m}$ um limitante superior das raízes de $p$. Sejam $i, j \in[m]$. Então

$$
E_{x=u}\left(\partial_{x_{j}}^{2} \Phi_{p}^{i}(x)\right) \geq 0 .
$$


Demonstração. Seja $d$ o grau de $\phi_{p, u}^{i}$. Se $i=j$, então $\partial_{x_{j}}^{2} \Phi_{p}^{i}(x)=\partial_{x_{i}}^{2} \Phi_{p}^{i}(x)$. Pela Proposição 8.6, temos que $E_{x=u}\left(\Phi_{p}^{i}(x)\right)=\sum_{k \in[d]} \frac{1}{u_{i}-r_{k}\left(\phi_{p, u}^{i}\right)}$. Assim

$$
\begin{aligned}
E_{x=u}\left(\partial_{x_{i}}^{2} \Phi_{p}^{i}(x)\right) & =E_{x_{i}=u_{i}}\left(\partial_{x_{i}}^{2} \sum_{k \in[d]} \frac{1}{x_{i}-r_{k}\left(\phi_{p, u}^{i}\right)}\right) \\
& =E_{x_{i}=u_{i}}\left(\sum_{k \in[d]} \frac{2}{\left(x_{i}-r_{k}\left(\phi_{p, u}^{i}\right)\right)^{3}}\right) \\
& =\sum_{k \in[d]} \frac{2}{\left(u_{i}-r_{k}\left(\phi_{p, u}^{i}\right)\right)^{3}} .
\end{aligned}
$$

Logo, como $u$ é um limitante superior das raízes de $p$, então $u_{i}>r_{k}\left(\phi_{p, u}^{i}\right)$ para todo $k \in[d]$ e, portanto, $E_{x=u}\left(\partial_{x_{j}}^{2} \Phi_{p}^{i}(x)\right)>0$.

Suponha que $i \neq j$. Pelo Lema 8.7, existem matrizes $A_{i}, A_{j} \in \mathbb{C}^{n \times n}$ positivas semidefinidas e $A \in \mathbb{C}^{n \times n}$ hermitiana, onde $B:=u_{i} A_{i}+u_{j} A_{j}+A$ é positiva definida e

$$
E_{x=u+\varepsilon e_{j}}\left(\partial_{x_{j}} \Phi_{p}^{i}(x)\right)=-\operatorname{Tr}\left(A_{j}\left(\varepsilon A_{j}+B\right)^{-1} A_{i}\left(\varepsilon A_{j}+B\right)^{-1}\right)
$$

para todo $\varepsilon \in \mathbb{R}_{+}$. Seja $\delta>0$ suficientemente pequeno tal que

$$
\rho\left(\delta A_{j} B^{-1}\right)<1 .
$$

Note que $\delta=1 /\left(1+\rho\left(A_{j} B^{-1}\right)\right)$ satisfaz essa desigualdade. Logo, vale que $I+\delta A_{j} B^{-1}$ é inversível. Como $u+\delta e_{j}$ também é um limitante superior das raízes de $p$, então vale que

$$
\begin{aligned}
E_{x=u+\delta e_{j}}\left(\partial_{x_{j}} \Phi_{p}^{i}(x)\right) & =-\operatorname{Tr}\left(A_{j}\left(\delta A_{j}+B\right)^{-1} A_{i}\left(\delta A_{j}+B\right)^{-1}\right) \\
& =-\operatorname{Tr}\left(A_{j} B^{-1}\left(I+\delta A_{j} B^{-1}\right)^{-1} A_{i} B^{-1}\left(I+\delta A_{j} B^{-1}\right)^{-1}\right) .
\end{aligned}
$$

Pela Proposição 2.4, temos que $\left(I+\delta A_{j} B^{-1}\right)^{-1}=\sum_{k \in \mathbb{N}}\left(-\delta A_{j} B^{-1}\right)^{k}$. Logo, pela Proposição 2.13 e por (8.4), vale que

$$
\begin{aligned}
E_{x=u} & \left(\partial_{x_{j}}^{2} \Phi_{p}^{i}(x)\right) \\
& =\lim _{\delta \rightarrow 0} \frac{E_{x=u+\delta e_{j}}\left(\partial_{x_{j}} \Phi_{p}^{i}(x)\right)-E_{x=u}\left(\partial_{x_{j}} \Phi_{p}^{i}(x)\right)}{\delta} \\
& =\lim _{\delta \rightarrow 0} \frac{-\operatorname{Tr}\left(A_{j} B^{-1} \sum_{k \in \mathbb{N}}\left(-\delta A_{j} B^{-1}\right)^{k} A_{i} B^{-1} \sum_{k \in \mathbb{N}}\left(-\delta A_{j} B^{-1}\right)^{k}\right)+\operatorname{Tr}\left(A_{j} B^{-1} A_{i} B^{-1}\right)}{\delta} .
\end{aligned}
$$

Assim, como

$$
\lim _{\delta \rightarrow 0} \frac{-\operatorname{Tr}\left(A_{j} B^{-1} \sum_{k \in \mathbb{N} \backslash\{0\}}\left(-\delta A_{j} B^{-1}\right)^{k} A_{i} B^{-1} \sum_{k \in \mathbb{N} \backslash\{0\}}\left(-\delta A_{j} B^{-1}\right)^{k}\right)}{\delta}=0,
$$

então vale que

$$
\begin{aligned}
E_{x=u}\left(\partial_{x_{j}}^{2} \Phi_{p}^{i}(x)\right) & =-\operatorname{Tr}\left(A_{j} B^{-1}(-1) A_{j} B^{-1} A_{i} B^{-1}+A_{j} B^{-1} A_{i} B^{-1}(-1) A_{j} B^{-1}\right) \\
& =\operatorname{Tr}\left(A_{j} B^{-1} A_{j} B^{-1} A_{i} B^{-1}\right)+\operatorname{Tr}\left(A_{j} B^{-1} A_{i} B^{-1} A_{j} B^{-1}\right) \\
& =2 \operatorname{Tr}\left(A_{j} B^{-1} A_{j} B^{-1} A_{i} B^{-1}\right) \\
& =2 \operatorname{Tr}\left(\left(A_{j} B^{-1} A_{j}\right)\left(B^{-1} A_{i} B^{-1}\right)\right) \geq 0,
\end{aligned}
$$

onde a desigualdade vale pela Proposição 2.43. 
A monotonicidade e a convexidade coordenada a coordenada, que foram as duas propriedades da função potencial mostradas acima, serão utilizadas neste texto para provar outras propriedades da função potencial que são fundamentais para construir iterativamente o Mixed Characteristic Polynomial limitando as raízes do polinômio em progresso por barreiras. Segundo uma dessas propriedades, ao aplicar o operador $\left(1-\partial_{x_{i}}\right)$ em um polinômio real estável $p \in \mathbb{R}\left[x_{1}, \ldots, x_{m}\right]$ cujo potencial associado a $i$-ésima coordenada é menor que 1 , todos os limitantes das raízes de $p$ são preservados. Em outras palavras, todos os limitantes das raízes de $p$ também são limitantes das raízes de $\left(1-\partial_{x_{i}}\right) p$. Para provar esse resultado, utilizamos a monotonicidade da função potencial, como mostrado a seguir.

Lema 8.10. Sejam $p \in \mathbb{R}\left[x_{1}, \ldots, x_{m}\right]$ um polinômio real estável e $i \in[m]$. Seja $u \in \mathbb{R}^{m}$ tal que $\Phi_{p}^{i}(u)<1$. Se $u$ é um limitante superior das raízes de $p$, então $u$ é um limitante superior das raízes de $\left(1-\partial_{x_{i}}\right) p$.

Demonstração. Seja $v \in \mathbb{R}_{+}^{m}$. Pelo Lema 8.8, temos que

$$
\Phi_{p}^{i}(u+v) \leq \cdots \leq \Phi_{p}^{i}\left(u+v_{1} e_{1}+v_{2} e_{2}\right) \leq \Phi_{p}^{i}\left(u+v_{1} e_{1}\right) \leq \Phi_{p}^{i}(u)<1 .
$$

Assim, pela definição de potenciais, vale que

$$
\Phi_{p}^{i}(u+v)=E_{x=u+v}\left(\frac{\partial_{x_{i}} p(x)}{p(x)}\right)<1,
$$

o que implica que $p(u+v)-E_{x=u+v}\left(\partial_{x_{i}} p(x)\right)>0$. Portanto, para todo $v \in \mathbb{R}_{+}^{m}$, vale que

$$
E_{x=u+v}\left(\left(1-\partial_{x_{i}}\right) p(x)\right)>0 .
$$

Mostraremos agora outra propriedade da função potencial que é fundamental para construir iterativamente o Mixed Characteristic Polynomial de modo a limitar as suas raízes a cada iteração. Sejam $p \in \mathbb{R}\left[x_{1}, \ldots, x_{m}\right]$ um polinômio real estável e $j \in[m]$. Seja $u \in \mathbb{R}^{m}$ um limitante superior das raízes de $p$ tal que o potencial $\Phi_{p}^{j}(u)$ é menor que (e afastado de) 1. Segundo essa propriedade, o potencial $\Phi_{\left(1-\partial_{x_{j}}\right) p}^{i}$ de um limitante maior que (e afastado de) $u$ na $j$-ésima coordenada é no máximo o potencial $\Phi_{p}^{i}$ de $u$ em todas as coordenadas $i$. Assim, à medida que a operação $\left(1-\partial_{x_{j}}\right)$ é aplicada em $p$, e à medida que aumentamos suficientemente o limitante superior de $p$, o potencial do polinômio resultante em relação a barreira resultante não aumenta. Utilizamos a monotonicidade e a convexidade da função potencial para provar esse resultado.

Teorema 8.11 (Marcus, Spielman e Srivastava [25]). Sejam $p \in \mathbb{R}\left[x_{1}, \ldots, x_{m}\right]$ um polinômio real estável, $j \in[m]$ e $u$ um limitante superior das raízes de $p$. Seja $\delta>0$ tal que $E_{x=u}\left(\Phi_{p}^{j}(x)\right) \leq 1-\frac{1}{\delta}$. Então

$$
E_{x=u+\delta e_{j}}\left(\Phi_{\left(1-\partial_{x_{j}}\right) p}^{i}(x)\right) \leq \Phi_{p}^{i}(u)
$$

para todo $i \in[m]$.

Demonstração. Seja $i \in[m]$. Pelo Lema 8.8, temos que $\Phi_{p}^{j}(u) \geq \Phi_{p}^{j}\left(u+\delta e_{j}\right)$, o que implica que

$$
\delta\left(1-\Phi_{p}^{j}(u)\right) \leq \delta\left(1-\Phi_{p}^{j}\left(u+\delta e_{j}\right)\right) .
$$

Logo, pelo Lema 8.8, temos que

$$
\delta\left(1-\Phi_{p}^{j}(u)\right) E_{x=u+\delta e_{j}}\left(-\partial_{x_{j}} \Phi_{p}^{i}(x)\right) \leq \delta\left(1-\Phi_{p}^{j}\left(u+\delta e_{j}\right)\right) E_{x=u+\delta e_{j}}\left(-\partial_{x_{j}} \Phi_{p}^{i}(x)\right) .
$$


Como $\Phi_{p}^{j}(u) \leq 1-\frac{1}{\delta}$, então $\delta\left(1-\Phi_{p}^{j}(u)\right) \geq 1$, o que implica que

$$
E_{x=u+\delta e_{j}}\left(\delta\left(1-\Phi_{p}^{j}(x)\right)\left(-\partial_{x_{j}} \Phi_{p}^{i}(x)\right)\right) \geq-E_{x=u+\delta e_{j}}\left(\partial_{x_{j}} \Phi_{p}^{i}(x)\right) .
$$

Logo, como $\Phi_{p}^{j}(u)<1$, então

$$
E_{x=u+\delta e_{j}}\left(\delta\left(-\partial_{x_{j}} \Phi_{p}^{i}(x)\right)\right) \geq \frac{-E_{x=u+\delta e_{j}}\left(\partial_{x_{j}} \Phi_{p}^{i}(x)\right)}{1-\Phi_{p}^{j}\left(u+\delta e_{j}\right)} .
$$

Pelo Lema 8.9, temos que $\Phi_{p}^{i}(x)$ é convexa. Assim, vale que

$$
E_{x=u+\delta e_{j}}\left(\delta\left(\partial_{x_{j}} \Phi_{p}^{i}(x)\right)\right) \geq \Phi_{p}^{i}\left(u+\delta e_{j}\right)-\Phi_{p}^{i}(u) .
$$

Assim, por (8.5), temos que

$$
\Phi_{p}^{i}(u)-\Phi_{p}^{i}\left(u+\delta e_{j}\right) \geq \frac{E_{x=u+\delta e_{j}}\left(-\partial_{x_{j}} \Phi_{p}^{i}(x)\right)}{1-\Phi_{p}^{j}\left(u+\delta e_{j}\right)} .
$$

Seja $z$ um limitante superior das raízes de $p$. Pelo Lema 8.10, temos que $z$ também é limitante das raízes de $p-\partial_{x_{j}} p$. Assim, vale que

$$
\begin{aligned}
E_{x=z}\left(\Phi_{p-\partial_{x_{j}} p}^{i}(x)\right) & =E_{x=z}\left(\frac{\partial_{x_{i}}\left(p-\partial_{x_{j}} p\right)}{p-\partial_{x_{j}} p}(x)\right) \\
& =E_{x=z}\left(\frac{\partial_{x_{i}}\left(\left(1-\Phi_{p}^{j}\right) p\right)}{\left(1-\Phi_{p}^{j}\right) p}(x)\right) \\
& =E_{x=z}\left(\frac{\left(1-\Phi_{p}^{j}\right) \partial_{x_{i}} p-\partial_{x_{i}} \Phi_{p}^{j} p}{\left(1-\Phi_{p}^{j}\right) p}(x)\right) .
\end{aligned}
$$

Logo, como $p\left(u+\delta e_{j}\right)>0$ e $\Phi_{p}^{j}\left(u+\delta e_{j}\right) \leq \Phi_{p}^{j}(u)<1$ (pelo Lema 8.8), então

$$
\begin{aligned}
E_{x=u+\delta e_{j}}\left(\Phi_{p-\partial_{x_{j}} p}^{i}(x)\right) & =\Phi_{p}^{i}\left(u+\delta e_{j}\right)-E_{x=u+\delta e_{j}}\left(\frac{\partial_{x_{i}} \Phi_{p}^{j}(x)}{1-\Phi_{p}^{j}(x)}\right) \\
& =\Phi_{p}^{i}\left(u+\delta e_{j}\right)-E_{x=u+\delta e_{j}}\left(\frac{\partial_{x_{i}} \frac{\partial_{x_{j}} p(x)}{p(x)}}{1-\Phi_{p}^{j}(x)}\right) \\
& =\Phi_{p}^{i}\left(u+\delta e_{j}\right)-E_{x=u+\delta e_{j}}\left(\frac{\partial_{x_{j}} \frac{\partial_{x_{i}} p(x)}{p(x)}}{1-\Phi_{p}^{j}(x)}\right) \\
& =\Phi_{p}^{i}\left(u+\delta e_{j}\right)-E_{x=u+\delta e_{j}}\left(\frac{\partial_{x_{j}} \Phi_{p}^{i}(x)}{1-\Phi_{p}^{j}(x)}\right) .
\end{aligned}
$$

Portanto, por (8.6), temos que

$$
\Phi_{p}^{i}(u)-\Phi_{p}^{i}\left(u+\delta e_{j}\right) \geq E_{x=u+\delta e_{j}}\left(\Phi_{p-\partial_{x_{j}} p}^{i}(x)\right)-\Phi_{p}^{i}\left(u+\delta e_{j}\right) .
$$

Utilizando as propriedades da função potencial apresentadas acima, mostraremos, na próxima seção, que as raízes do Mixed Characteristic Polynomial de matrizes positivas semidefinidas que somam para a identidade são limitadas por um valor desejado. 


\subsection{Resolução do problema de Kadison-Singer}

Nesta seção, mostraremos a prova de uma solução do problema de Kadison-Singer, apresentada por Marcus, Spielman e Srivastava, provando uma de suas conjecturas. Primeiramente, apresentaremos um resultado intermediário (Teorema 8.13) que mostra a probabilidade não nula das raízes de uma variável aleatória que assume valores em $\mathbb{C}[x]$ serem limitadas superiormente por um valor desejado. Para provar esse resultado, utilizamos propriedades de árvores entrelaçadoras em polinômios cuja soma de suas folhas é o Mixed Characteristic Polynomial de certas matrizes positivas semidefinidas que somam para a identidade. Dessa forma, é possível limitar as raízes de pelo menos um polinômio folha dessa árvore entrelaçadora de polinômios pela raiz máxima do Mixed Characteristic Polynomial que, por sua vez, é limitada por um valor desejado. O Teorema 8.12 fornece esse limitante e sua prova consiste no uso da técnica de construção iterativa do Mixed Characteristic Polynomial com o auxílio de barreiras e da função potencial. Segue a prova desse resultado que utiliza uma das propriedades de Mixed Characteristic Polynomials e as propriedades da função potencial apresentadas na seção anterior.

Teorema 8.12. Sejam $\varepsilon>0$ e $A_{1}, \ldots, A_{m} \in \mathbb{C}^{n \times n}$ matrizes positivas semidefinidas tais que $\sum_{i \in[m]} A_{i}=I$ e $\operatorname{Tr}\left(A_{i}\right) \leq \varepsilon$ para todo $i \in[m]$. Então

$$
r_{\max }\left(\mu\left[A_{1}, \ldots, A_{m}\right]\right) \leq(1+\sqrt{\varepsilon})^{2} .
$$

Demonstração. Seja $x$ uma indeterminada. Defina $\bar{\mu} \in \mathbb{C}\left[z_{1}, \ldots, z_{m}\right] \operatorname{como} \bar{\mu}(z):=\operatorname{det}\left(\sum_{i \in[m]} z_{i} A_{i}\right)$. Pelo Corolário 5.16, vale que $\bar{\mu} \in \mathbb{R}\left[z_{1}, \ldots, z_{m}\right]$. Além disso, pelo Teorema 8.2, temos que

$$
\mu\left[A_{1}, \ldots, A_{m}\right](x)=E_{z=x \mathbb{1}}\left(\left(\prod_{j \in[m]}\left(1-\partial_{z_{i}}\right)\right) \bar{\mu}(z)\right) .
$$

Seja $\alpha:=\sqrt{\varepsilon}+\varepsilon$. Note que $\bar{\mu}(\alpha \mathbb{1})=\operatorname{det}\left(\sum_{i \in[m]} \alpha A_{i}\right)=\alpha^{n} \operatorname{det}(I)=\alpha^{n}$. Como $A_{i}$ é positiva semidefinida para todo $i \in[m]$, então, para todo $v \in \mathbb{R}_{+}^{n}$, vale que

$$
\bar{\mu}(\alpha \mathbb{1}+v)=\operatorname{det}\left(\sum_{i \in[m]}\left(\alpha+v_{i}\right) A_{i}\right)=\operatorname{det}\left(\alpha I+\sum_{i \in[m]} v_{i} A_{i}\right) \geq \alpha^{n}>0 .
$$

Portanto, $\alpha \mathbb{1}$ é um limitante superior das raízes de $\bar{\mu}$.

Note que, para todo $j \in[m]$, vale que

$$
\begin{aligned}
\Phi_{\bar{\mu}}^{j}(\alpha \mathbb{1}) & =E_{z=\alpha \mathbb{1}}\left(\frac{\partial_{z_{j}} \bar{\mu}(z)}{\bar{\mu}(z)}\right) \\
& =\operatorname{Tr}\left(\left(\sum_{i \in[m]}\left(\alpha A_{i}\right)\right)^{-1} A_{j}\right) \\
& =\frac{1}{\alpha} \operatorname{Tr}\left(A_{j}\right) \\
& \leq \frac{\varepsilon}{\sqrt{\varepsilon}+\varepsilon} .
\end{aligned}
$$

Defina $\bar{\mu}_{\ell}(z):=\left(\prod_{j \in[\ell]}\left(1-\partial_{z_{j}}\right)\right) \bar{\mu}(z)$ para todo $\ell \in\{0, \ldots, m\}$. Seja $\delta:=1+\sqrt{\varepsilon}$. Defina também $u_{\ell}:=\alpha \mathbb{1}+\delta \sum_{i \in[\ell]} e_{i}$. Provaremos por indução em $\ell$ que $\bar{\mu}_{\ell}$ é real estável, que $u_{\ell}$ é um limitante superior das raízes de $\bar{\mu}_{\ell}$ e que

$$
\Phi_{\bar{\mu}_{\ell}}^{j}\left(u_{\ell}\right) \leq \frac{\varepsilon}{\sqrt{\varepsilon}+\varepsilon}
$$


para todo $j \in[m]$.

Note que $\bar{\mu}_{0}(z)=\bar{\mu}(z)$ e $u_{0}=\alpha \mathbb{1}$. Pelo Corolário 5.16, temos que $\bar{\mu}_{0}$ é real estável. Assim, se $\ell=0$, é fácil ver que a hipótese vale por (8.8).

Suponha que $\ell \geq 1$. Pela hipótese de indução, temos que $\bar{\mu}_{\ell-1}$ é real estável, que $u_{\ell-1}$ é um limitante superior das raízes de $\bar{\mu}_{\ell-1}$ e que

$$
\Phi_{\bar{\mu}_{\ell-1}}^{j}\left(u_{\ell-1}\right) \leq \frac{\varepsilon}{\sqrt{\varepsilon}+\varepsilon}=1-\frac{1}{\delta}
$$

para todo $j \in[m]$. Como $\bar{\mu}_{\ell-1}$ é real estável, então, pelo Corolário 5.14, vale que $\bar{\mu}_{\ell}$ também é real estável. Além disso, pelo Lema 8.10, temos que $u_{\ell-1}$ é um limitante superior das raízes de $\bar{\mu}_{\ell}$, o que implica que $u_{\ell}$ também é um limitante superior das raízes de $\bar{\mu}_{\ell}$. Assim, as condições do Teorema 8.11 são satisfeitas, o que implica que

$$
\Phi_{\bar{\mu}_{\ell}}^{j}\left(u_{\ell}\right) \leq \Phi_{\bar{\mu}_{\ell-1}}^{j}\left(u_{\ell-1}\right) \leq \frac{\varepsilon}{\sqrt{\varepsilon}+\varepsilon}
$$

para todo $j \in[m]$. Portanto, vale que $(\alpha+\delta) \mathbb{1}$ é um limitante superior das raízes de

$$
\left(\prod_{j \in[m]}\left(1-\partial_{z_{j}}\right)\right) \bar{\mu}(z)
$$

Dessa forma, por (8.7), temos que todas as raízes de $\mu\left[A_{1}, \ldots, A_{m}\right](x)$ são no máximo $\alpha+\delta=$ $(1+\sqrt{\varepsilon})^{2}$.

No resultado acima, limitamos as raízes do Mixed Characteristic Polynomial de matrizes positivas semidefinidas $A_{1}, \ldots, A_{m} \in \mathbb{C}^{n \times n}$ que somam para a identidade utilizando a representação do Mixed Characteristic Polynomial apresentada no Teorema 8.2:

$$
\mu\left[A_{1}, \ldots, A_{m}\right](x)=E_{z=x \mathbb{1}}\left(\left(\prod_{i \in[m]}\left(1-\partial_{z_{i}}\right)\right) \bar{\mu}(z)\right) .
$$

Primeiramente, encontramos um limitante superior $\alpha \mathbb{1}$ para as raízes do polinômio real estável multivariado $\bar{\mu}:=\operatorname{det}\left(\sum_{i \in[m]} z_{i} A_{i}\right)$. Assim, podemos considerar $\alpha \mathbb{1}$ como a barreira inicial de $\bar{\mu}$. A partir de $\bar{\mu}$, construímos o Mixed Characteristic Polynomial de $A_{1}, \ldots, A_{m}$ em $m$ iterações. A cada iteração $i$, é aplicado o operador $\left(1-\partial_{x_{i}}\right)$ ao polinômio resultante e é somado um valor $\delta$ à $i$-ésima coordenada da barreira em progresso, onde $\delta$ é o mesmo para todas as iterações. Note que os polinômios em progresso são reais estáveis, pois os operadores $\left(1-\partial_{x_{i}}\right)$ preservam a estabilidade real. Pelos resultados mostrados na seção de barreiras e função potencial, o potencial dos polinômios em progresso diminui a cada iteração e suas raízes são limitadas superiormente pelas barreiras referentes à iteração em questão. Assim, o polinômio referente a $m$-ésima iteração é

$$
\left(\prod_{i \in[m]}\left(1-\partial_{z_{i}}\right)\right) \bar{\mu}(z)
$$

e suas raízes são limitadas superiormente pela barreira $\alpha+\delta \mathbb{1}$. Como os valores de todas as coordenadas dessa barreira são iguais a $\alpha+\delta$, todas as raízes de $\left(\prod_{i \in[m]}\left(1-\partial_{z_{i}}\right)\right) \bar{\mu}(z)$ são menores que $\alpha+\delta$ em pelo menos uma coordenada, o que implica que as raízes de $\mu\left[A_{1}, \ldots, A_{m}\right](x)$ são limitadas por $\alpha+\delta$. Utilizamos esse resultado para limitar as raízes de pelo menos um polinômio que compõe uma árvore entrelaçadora de polinômios cuja soma de suas folhas é um Mixed Characteristic Polynomial, como mostrado a seguir. 


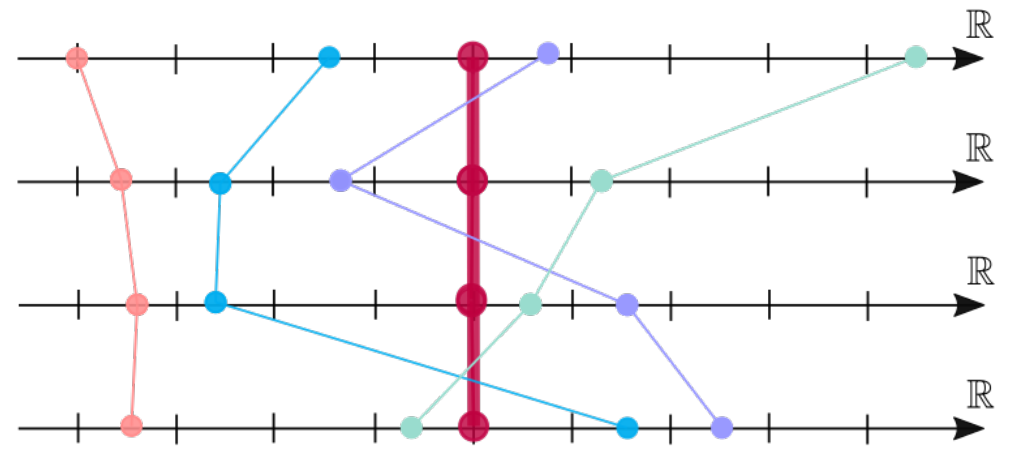

- limitante das raízes de $p$
三 raízes de $p$

Figura 8.2: Representação de um limitante das raízes de um polinômio $p$ cujos valores de todas as coordenadas são iguais. Cada eixo representa uma dimensão.

Sejam $v_{1}, \ldots, v_{m}$ variáveis aleatórias de suporte finito que assumem valores em $\mathbb{C}^{n}$ e seja $R:=$ $R_{v_{1}} \times \cdots \times R_{v_{m}}$. Defina $A_{i}:=\mathbb{E}_{v_{i}} v_{i} v_{i}^{*}$ para todo $i \in[m]$. Pelo Corolário 5.29, temos que

$$
\mu\left[A_{1}, \ldots, A_{m}\right](x)=\sum_{w \in R} q_{v_{1}, \ldots, v_{m}}[w](x) .
$$

Além disso, pelo Teorema 5.32, vale que a família $\left(q_{v_{1}, \ldots, v_{m}}[w]\right)_{w \in R}$ forma uma árvore entrelaçadora de polinômios. Assim, as raízes de pelo menos um dos polinômios dessa família são limitadas superiormente por $\alpha+\delta$, que é a raiz máxima do Mixed Characteristic Polynomial de $A_{1}, \ldots, A_{m}$. Seja $w \in R$ tal que as raízes de $q_{v_{1}, \ldots, v_{m}}[w]$ são limitadas por $\alpha+\delta$. Como o polinômio característico de $\sum_{i \in[m]} w_{i} w_{i}^{*}$ é o polinômio $q_{v_{1}, \ldots, v_{m}}[w]$ multiplicado por um escalar, então as raízes do polinômio característico de $\sum_{i \in[m]} w_{i} w_{i}^{*}$ também são limitadas superiormente por $\alpha+\delta$. Dessa forma, os autovalores da matriz $\sum_{i \in[m]} w_{i} w_{i}^{*}$ são limitados superiormente por $\alpha+\delta$, como mostrado a seguir.

Teorema 8.13 (Marcus, Spielman e Srivastava [25]). Sejam $\varepsilon>0$ e $v_{1}, \ldots, v_{m}$ variáveis aleatórias independentes que assumem valores em $\mathbb{C}^{n}$ com $R_{v_{i}}$ finito para todo $i \in[m]$. Se

$$
\sum_{i \in[m]} \mathbb{E}_{v_{i}}\left(v_{i} v_{i}^{*}\right)=I
$$

e $\mathbb{E}_{v_{i}}\left(\left\|v_{i}\right\|_{2}^{2}\right) \leq \varepsilon$ para todo $i \in[m]$, então

$$
\mathbb{P}\left[\left\|\sum_{i \in[m]} v_{i} v_{i}^{*}\right\|_{2} \leq(1+\sqrt{\varepsilon})^{2}\right]>0 .
$$

Demonstração. Defina $R:=R_{v_{1}} \times \cdots \times R_{v_{m}}$ e $A_{i}:=\mathbb{E}_{v_{i}} v_{i} v_{i}^{*}$ para todo $i \in[m]$. Pelo Teorema 5.32, temos que a família de polinômios $\left(q_{v_{1}, \ldots, v_{m}}[w]\right)_{w \in R}$ forma uma árvore entrelaçadora de polinômios. Assim, pelo Corolário 4.12, temos que existe $y \in R$ tal que

$$
r_{\max }\left(q_{v_{1}, \ldots, v_{m}}[y]\right) \leq r_{\max }\left(\sum_{w \in R} q_{v_{1}, \ldots, v_{m}}[w]\right) .
$$


Pela definição de $q_{v_{1}, \ldots, v_{m}}$, temos que

$$
\begin{aligned}
\sum_{w \in R} q_{v_{1}, \ldots, v_{m}}[w] & =\mathbb{E}_{v_{1}, \ldots, v_{m}} \operatorname{det}\left(x I-\sum_{i \in[m]} v_{i} v_{i}^{*}\right) \\
& =\mu\left[A_{1}, \ldots, A_{m}\right](x)
\end{aligned}
$$

pelo Corolário 5.29.

Logo, existe $y \in R$ tal que

$$
r_{\max }\left(q_{v_{1}, \ldots, v_{m}}[y]\right) \leq r_{\max }\left(\mu\left[A_{1}, \ldots, A_{m}\right]\right) .
$$

Como $q_{v_{1}, \ldots, v_{m}}[y]=\left(\prod_{i \in[m]} \mathbb{P}\left(v_{i}=y_{i}\right)\right) \operatorname{det}\left(x I-\sum_{i \in[m]} y_{i} y_{i}^{*}\right)$ por definição, então

$$
r_{\max }\left(q_{v_{1}, \ldots, v_{m}}[y]\right)=r_{\max }\left(\operatorname{det}\left(x I-\sum_{i \in[m]} y_{i} y_{i}^{*}\right)\right) .
$$

Dessa forma, pelo Teorema 8.12, temos que

$$
r_{\max }\left(\operatorname{det}\left(x I-\sum_{i \in[m]} y_{i} y_{i}^{*}\right)\right) \leq r_{\max }\left(\mu\left[A_{1}, \ldots, A_{m}\right]\right) \leq(1+\sqrt{\varepsilon})^{2},
$$

o que implica que

$$
\left\|\sum_{i \in[m]} y_{i} y_{i}^{*}\right\|_{2} \leq(1+\sqrt{\varepsilon})^{2} .
$$

O resultado mostrado acima implica um resultado intermediário que, por sua vez, implica uma das conjecturas da solução positiva do Problema de Kadison-Singer. Sejam $u_{1}, \ldots, u_{m} \in \mathbb{C}^{n}$ vetores de norma pequena e seja $\alpha \in \mathbb{N}$ com $\alpha \leq m$. Defina $U:=\left\{u_{1}, \ldots, u_{m}\right\}$. Segundo o resultado intermediário (Corolário 8.14), existe uma partição $\left\{S_{1}, \ldots, S_{\alpha}\right\}$ do conjunto $[m]$ tal que, para todo $k \in[\alpha]$, os autovalores da matriz $\sum_{i \in S_{k}} u_{i} u_{i}^{*}$ são limitados superiormente por um valor que depende de $\alpha$ e das normas dos vetores pertencentes a $U$. Sejam $v_{1}, \ldots, v_{m}$ variáveis aleatórias independentes que assumem valores em $\mathbb{C}^{\alpha n}$. A intuição da prova desse resultado é que as variáveis aleatórias sejam escolhidas de forma que cada variável aleatória $v_{i}$ assume valores $w_{i}[\beta]$ para todo $\beta \in[\alpha]$ de modo que

$$
\left\|\sum_{j \in S} u_{j} u_{j}^{*}\right\|_{2}=\left\|\sum_{j \in S} w_{j}[\beta] w_{j}[\beta]^{*}\right\|_{2}
$$

para todo $S \subseteq[m]$ e todo $\beta \in[\alpha]$. Em outras palavras, associamos cada variável aleatória a um elemento de $U$ e tipificamos os valores que cada variável aleatória pode assumir com números de 1 a $\alpha$. Essa tipificação é feita de forma que, ao agruparmos variáveis aleatórias em qualquer conjunto $S$, a norma de $\sum_{j \in S} w_{j}[\beta] w_{j}[\beta]^{*}$ é a mesma para todas as tipificações $\beta \in[\alpha]$. Além disso, a norma de $\sum_{j \in S} w_{j}[\beta] w_{j}[\beta]^{*}$, para qualquer tipificação $\beta$, também é a mesma norma de $\sum_{j \in S} u_{j} u_{j}^{*}$. Utilizamos o Teorema 8.13 para mostrar que cada variável aleatória $v_{i}$ está associada a um tipo $f:\left\{v_{1}, \ldots, v_{m}\right\} \rightarrow[\alpha]$ de forma que os autovalores da matriz $\sum_{i \in[m]} w_{i}\left[f\left(v_{i}\right)\right] w_{i}\left[f\left(v_{i}\right)\right]^{*}$ são limitados por um valor desejado. Seja $\left\{S_{1}, \ldots, S_{\alpha}\right\}$ uma partição de $[m]$ tal que $i \in S_{f\left(v_{i}\right)}$ para todo $i \in[m]$. Por (8.9), temos que, para todo $\beta \in[\alpha]$, vale que

$$
\left\|\sum_{i \in S_{\beta}} u_{i} u_{i}^{*}\right\|_{2}=\left\|\sum_{i \in S_{\beta}} w_{i}\left[f\left(v_{i}\right)\right] w_{i}\left[f\left(v_{i}\right)\right]^{*}\right\|_{2} \leq\left\|\sum_{i \in[m]} w_{i}\left[f\left(v_{i}\right)\right] w_{i}\left[f\left(v_{i}\right)\right]^{*}\right\|_{2},
$$

o que implica que os autovalores de $\sum_{i \in S_{\beta}} u_{i} u_{i}^{*}$ também são limitados por um valor desejado. 
Corolário 8.14. Seja $\varepsilon>0$ e sejam $u_{1}, \ldots, u_{m} \in \mathbb{C}^{n}$ tais que $\sum_{i \in[m]} u_{i} u_{i}^{*}=I$ e $\left\|u_{i}\right\|_{2}^{2} \leq \varepsilon$ para todo $i \in[m]$. Seja $\alpha \in \mathbb{N} \operatorname{com} \alpha \leq m$. Então existe uma partição $\left\{S_{1}, \ldots, S_{\alpha}\right\}$ de $[m]$ tal que

$$
\left\|\sum_{i \in S_{\beta}} u_{i} u_{i}^{*}\right\|_{2} \leq\left(\frac{1}{\sqrt{\alpha}}+\sqrt{\varepsilon}\right)^{2}
$$

para todo $\beta \in[\alpha]$.

Demonstração. Defina $w_{i}[\beta] \in \mathbb{C}^{\alpha n}$ como

$$
w_{i}[\beta]:=0^{n(\beta-1)} \oplus u_{i} \oplus 0^{n(\alpha-\beta)}=\underbrace{0^{n} \oplus \cdots \oplus 0^{n}}_{\beta-1 \text { vezes }} \oplus u_{i} \oplus \underbrace{0^{n} \oplus \cdots \oplus 0^{n}}_{\alpha-\beta \text { vezes }}
$$

para todos $i \in[m]$ e $\beta \in[\alpha]$.

Sejam $v_{1}, \ldots, v_{m}$ variáveis aleatórias independentes com distribuição uniforme que assumem valores em $\mathbb{C}^{\alpha n}$ tais que $R_{v_{i}}=\left\{\sqrt{\alpha} w_{i}[1], \ldots, \sqrt{\alpha} w_{i}[\alpha]\right\}$ para todo $i \in[m]$.

Seja $i \in[m]$. Como a distribuição de $v_{i}$ é uniforme, então

$$
\mathbb{E} v_{i} v_{i}^{*}[\{(k-1) n+1, \ldots, k n\},\{(\ell-1) n+1, \ldots, \ell n\}]= \begin{cases}u_{i} u_{i}^{*} & \text { se } k=\ell \\ 0^{d \times d} & \text { se } k \neq \ell\end{cases}
$$

para todos $k, \ell \in[\alpha]$. Assim, vale que

$$
\mathbb{E} v_{i} v_{i}^{*}=\left[\begin{array}{cccc}
u_{i} u_{i}^{*} & 0^{n \times n} & \cdots & 0^{n \times n} \\
0^{n \times n} & u_{i} u_{i}^{*} & \ddots & \vdots \\
\vdots & \ddots & \ddots & 0^{n \times n} \\
0^{n \times n} & \cdots & 0^{n \times n} & u_{i} u_{i}^{*}
\end{array}\right]
$$

o que implica que

$$
\sum_{j \in[m]} \mathbb{E} v_{j} v_{j}^{*}=\left[\begin{array}{cccc}
\sum_{j \in[m]} u_{j} u_{j}^{*} & 0^{n \times n} & \cdots & 0^{n \times n} \\
0^{n \times n} & \sum_{j \in[m]} u_{j} u_{j}^{*} & \ddots & \vdots \\
\vdots & \ddots & \ddots & 0^{n \times n} \\
0^{n \times n} & \cdots & 0^{n \times n} & \sum_{j \in[m]} u_{j} u_{j}^{*}
\end{array}\right]=I .
$$

Note que $\mathbb{E}\left\|v_{j}\right\|_{2}^{2}=\alpha\left\|u_{j}\right\|_{2}^{2} \leq \alpha \varepsilon$ para todo $j \in[m]$. Logo, pelo Teorema 8.13, temos que

$$
\mathbb{P}\left[\left\|\sum_{j \in[m]} v_{j} v_{j}^{*}\right\|_{2} \leq(1+\sqrt{\alpha \varepsilon})^{2}\right]>0,
$$

o que implica que existem $\beta_{1}, \ldots, \beta_{m} \in[\alpha]$ tais que

$$
\left\|\sum_{j \in[m]} \alpha w_{j}\left[\beta_{j}\right] w_{j}\left[\beta_{j}\right]^{*}\right\|_{2} \leq(1+\sqrt{\alpha \varepsilon})^{2} .
$$

Defina $S_{\beta}:=\left\{j: \beta_{j}=\beta\right\}$ para todo $\beta \in[\alpha]$. Note que

$$
\left\|\sum_{j \in S_{\beta}} u_{j} u_{j}^{*}\right\|_{2}=\left\|\sum_{j \in S_{\beta}} w_{j}\left[\beta_{j}\right] w_{j}\left[\beta_{j}\right]^{*}\right\|_{2}
$$




$$
\begin{aligned}
& =\frac{1}{\alpha}\left\|\sum_{j \in S_{\beta}} \alpha w_{j}\left[\beta_{j}\right] w_{j}\left[\beta_{j}\right]^{*}\right\|_{2} \\
& \leq \frac{1}{\alpha}\left\|\sum_{j \in[m]} \alpha w_{j}\left[\beta_{j}\right] w_{j}\left[\beta_{j}\right]^{*}\right\|_{2} \\
& \leq \frac{(1+\sqrt{\alpha \varepsilon})^{2}}{\alpha} \\
& =\left(\frac{1}{\sqrt{\alpha}}+\sqrt{\varepsilon}\right)^{2} .
\end{aligned}
$$

Note que, para todo $\beta \in[\alpha]$ e para todo $S \subseteq S_{\beta}$, vale que

$$
\left\|\sum_{j \in S} u_{j} u_{j}^{*}\right\|_{2} \leq\left\|\sum_{j \in S_{\beta}} u_{j} u_{j}^{*}\right\|_{2} \leq\left(\frac{1}{\sqrt{\alpha}}+\sqrt{\varepsilon}\right)^{2} .
$$

Portanto, como $\alpha \leq m$, então existe uma partição $\left\{S_{1}, \ldots, S_{\alpha}\right\}$ de $[m]$ tal que

$$
\left\|\sum_{j \in S_{i}} u_{j} u_{j}^{*}\right\|_{2} \leq\left(\frac{1}{\sqrt{\alpha}}+\sqrt{\varepsilon}\right)^{2}
$$

para todo $i \in[\alpha]$.

O resultado apresentado acima implica em uma das conjecturas equivalentes ao Problema de Kadison-Singer formulada por Weaver [30]. Segundo essa conjectura, existem duas constantes universais reais positivas $\gamma$ e $\theta$ tais que podemos dividir em duas partes não nulas $V_{1}$ e $V_{2}$ qualquer conjunto finito $V \subset \mathbb{C}^{n}$ de vetores de norma limitada com $\sum_{u \in V} u u^{*}=\gamma I$ de forma que

$$
\sum_{u \in V_{1}} u u^{*} \preceq(\gamma-\theta) I \quad \text { e } \quad \sum_{u \in V_{2}} u u^{*} \preceq(\gamma-\theta) I .
$$

Em outras palavras, existem dois valores reais positivos $\gamma$ e $\theta$ tais que qualquer conjunto de matrizes positivas semidefinidas de posto $1 \mathrm{em} \mathbb{C}^{n \times n}$ com norma limitada, escolhido de forma que todos os autovalores da soma dessas matrizes sejam iguais a $\gamma$, pode ser dividido em duas partes não nulas de forma que os autovalores da soma das matrizes de cada parte sejam limitados superiormente por $\gamma-\theta$. Mostraremos a seguir a existência dessas constantes universais $\gamma$ e $\theta$ utilizando o Corolário 8.14. O resultado foi enunciado novamente com o intuito de facilitar a leitura.

Conjectura 8.15 (Weaver [30]). Existem reais $\gamma>2$ e $\theta>0$ tais que, para todo inteiro $m \geq 2$ e todos $u_{1}, \ldots, u_{m} \in \mathbb{C}^{n}$ com $\left\|u_{i}\right\|_{2} \leq 1$ para todo $i \in[m]$, vale que, se

$$
\sum_{i \in[m]} u_{i} u_{i}^{*}=\gamma I
$$

então existe uma partição $\left\{S_{1}, S_{2}\right\}$ de $[m]$ tal que $\sum_{i \in S_{j}} u_{i} u_{i}^{*} \preceq(\gamma-\theta) I$ para todo $j \in[2]$.

Demonstração. Defina $\gamma:=18$ e $\theta:=2$. Defina também $\alpha:=2$ e $\varepsilon:=1 / \gamma$. Sejam $u_{1}, \ldots, u_{m} \in \mathbb{C}^{n}$ com $\left\|u_{i}\right\|_{2} \leq 1$ para todo $i \in[m]$. Suponha que $\sum_{i \in[m]} u_{i} u_{i}^{*}=\gamma I$. Seja $w:[m] \rightarrow \mathbb{C}^{n}$ definida como $i \mapsto \sqrt{\varepsilon} u_{i}$. Note que $\left\|w_{i}\right\|_{2} \leq \sqrt{\varepsilon}$ para todo $i \in[m]$ e $\sum_{i \in[m]} w_{i} w_{i}^{*}=I$. Como $w_{1}, \ldots, w_{m}$ satisfazem as condições do Corolário 8.14, temos que existe uma partição $\left\{S_{1}, S_{2}\right\}$ de $[m]$ tal que

$$
\left\|\sum_{i \in S_{j}} w_{i} w_{i}^{*}\right\|_{2} \leq\left(\frac{1}{\sqrt{\alpha}}+\sqrt{\varepsilon}\right)^{2}
$$


para todo $j \in[2]$. Logo, vale que

$$
\frac{1}{\gamma}\left\|\sum_{i \in S_{j}} u_{i} u_{i}^{*}\right\|_{2} \leq\left(\frac{1}{\sqrt{\alpha}}+\sqrt{\varepsilon}\right)^{2}
$$

para todo $j \in[2]$. Portanto

$$
\left\|\sum_{i \in S_{j}} u_{i} u_{i}^{*}\right\|_{2} \leq \gamma\left(\frac{1}{\sqrt{\alpha}}+\sqrt{\varepsilon}\right)^{2}=\left(\frac{\sqrt{\gamma}}{\sqrt{\alpha}}+1\right)^{2}=\left(\frac{3 \sqrt{2}}{\sqrt{2}}+1\right)^{2}=16=\gamma-\theta .
$$

Na prova do resultado acima, atribuímos um valor a $\gamma$ e $\theta$ e provamos que o resultado vale para esses valores. Porém, existem outras combinações dessas constantes universais $\gamma$ e $\theta$ que também satisfazem os resultados da Conjectura 8.15. Basta que $\gamma$ e $\theta$ satisfaçam as seguintes condições:

$$
\gamma>2, \quad \theta>0 \quad \text { e } \quad\left(\sqrt{\frac{\gamma}{2}}+1\right)^{2} \leq \gamma-\theta
$$




\section{Bibliografia}

[1] N. Alon e V. D. Milman. " $\lambda_{1}$, isoperimetric inequalities for graphs, and superconcentrators". Em: Journal of Combinatorial Theory, Series B 38.1 (1985), pp. 73-88. URL: http://dx . doi.org/10.1016/0095-8956(85)90092-9.

[2] J. Batson, D. A. Spielman e N. Srivastava. "Twice-Ramanujan sparsifiers". Em: SIAM Journal on Computing 41.6 (2012), pp. 1704-1721. URL: http://dx.doi.org/10.1137/090772873.

[3] A. A. Benczúr e D. R. Karger. "Approximating $s$ - $t$ minimum cuts in Õ $\left(n^{2}\right)$ time". Em: Proceedings of the Twenty-eighth Annual ACM Symposium on Theory of Computing. STOC '96. Philadelphia, Pennsylvania, USA: ACM, 1996, pp. 47-55. URL: http://doi .acm.org/10 . $1145 / 237814.237827$.

[4] Y. Bilu e N. Linial. "Lifts, discrepancy and nearly optimal spectral gap". Em: Combinatorica 26.5 (2006), pp. 495-519. URL: http://dx.doi.org/10.1007/s00493-006-0029-7.

[5] J. Borcea e P. Brändén. "Multivariate Pólya-Schur classification problems in the Weyl algebra". Em: Proceedings of the London Mathematical Society 101.1 (2010), pp. 73-104. URL: http://dx.doi.org/10.1112/plms/pdp049.

[6] P. G. Casazza et al. "Projections and the Kadison-Singer Problem". Em: Operators and Matrices 1.3 (2007), pp. 391-408.

[7] J. Cheeger. "A lower bound for the smallest eigenvalue of the Laplacian". Em: Problems in analysis (Papers dedicated to Salomon Bochner, 1969). Princeton University Press, Princeton, N. J., 1970, pp. 195-199.

[8] M. Chudnovsky e P. Seymour. "The roots of the independence polynomial of a clawfree graph". Em: Journal of Combinatorial Theory, Series B 97.3 (2007), pp. 350-357. URL: http://www. sciencedirect.com/science/article/pii/S0095895606000876.

[9] J. P. Dedieu. "Obreschkoff's theorem revisited: What convex sets are contained in the set of hyperbolic polynomials?" Em: Journal of pure and applied algebra 81.3 (1992), pp. 269-278. URL: http://wWw.sciencedirect.com/science/article/pii/002240499290060S.

[10] H. Fell. "On the zeros of convex combinations of polynomials". Em: Pacific Journal of Mathematics 89.1 (1980), pp. 43-50.

[11] C. D. Godsil. "Matchings and walks in graphs". Em: Journal of Graph Theory 5.3 (1981), pp. 285-297. URL: https://doi.org/10.1002/jgt.3190050310.

[12] C. D. Godsil e I. Gutman. "On the matching polynomial of a graph". Em: Algebraic methods in graph theory, Vol. I, II (Szeged, 1978). Vol. 25. Colloq. Math. Soc. János Bolyai. NorthHolland, Amsterdam-New York, 1981, pp. 241-249.

[13] Y. Greenberg. "On the spectrum of graphs and their universal covering". Tese de dout. Hebrew University, 1995. 
[14] P. R. Halmos. Finite-dimensional vector spaces. Courier Dover Publications, 2017.

[15] N. J. A. Harvey. An introduction to the Kadison-Singer Problem and the Paving Conjecture. 2013. URL: https://www.cs.ubc.ca/〜nickhar/papers/KS/KS.pdf.

[16] O. J. Heilmann e E. H. Lieb. "Theory of monomer-dimer systems". Em: Communications in Mathematical Physics 25 (1972), pp. 190-232. URL: http://projecteuclid.org/euclid. cmp/1103857921.

[17] S. Hoory, N. Linial e A. Wigderson. "Expander graphs and their applications". Em: American Mathematical Society. Bulletin. New Series 43.4 (2006), pp. 439-561. URL: http://dx.doi. org/10.1090/S0273-0979-06-01126-8.

[18] R. A. Horn e C. R. Johnson. Matrix analysis. Cambridge University Press, 2012.

[19] R. V. Kadison e I. M. Singer. "Extensions of pure states". Em: American Journal of Mathematics 81 (1959), pp. 383-400. URL: http://dx.doi.org/10.2307/2372748.

[20] R. Kannan, S. Vempala e A. Vetta. "On Clusterings: Good, Bad and Spectral". Em: Journal of the ACM 51.3 (2004), pp. 497-515. URL: http://doi.acm.org/10.1145/990308.990313.

[21] M. Krivelevich e B. Sudakov. "Pseudo-random graphs". Em: More sets, graphs and numbers. Springer, 2006, pp. 199-262.

[22] A. Lewis, P. Parrilo e M. Ramana. "The Lax conjecture is true". Em: Proceedings of the American Mathematical Society 133.9 (2005), pp. 2495-2499. URL: https://doi .org/10. 1090/S0002-9939-05-07752-X.

[23] A. Lubotzky, R. Phillips e P. Sarnak. "Ramanujan graphs". Em: Combinatorica 8.3 (1988), pp. 261-277. URL: http://dx.doi.org/10.1007/BF02126799.

[24] A. W. Marcus, D. A. Spielman e N. Srivastava. "Interlacing families I: Bipartite Ramanujan graphs of all degrees". Em: Annals of Mathematics. Second Series 182.1 (2015), pp. 307-325. URL: http://dx.doi.org/10.4007/annals.2015.182.1.7.

[25] A. W. Marcus, D. A. Spielman e N. Srivastava. "Interlacing families II: Mixed characteristic polynomials and the Kadison-Singer Problem". Em: Annals of Mathematics. Second Series 182.1 (2015), pp. 327-350. URL: http://dx.doi.org/10.4007/annals.2015.182.1.8.

[26] G. A. Margulis. "Explicit group-theoretic constructions of combinatorial schemes and their applications in the construction of expanders and concentrators". Em: Problemy Peredachi Informatsii 24.1 (1988), pp. 51-60.

[27] A. Nilli. "On the second eigenvalue of a graph". Em: Discrete Mathematics 91.2 (1991), pp. 207-210. URL: http://dx.doi.org/10.1016/0012-365X(91)90112-F.

[28] Q. I. Rahman e G. Schmeisser. Analytic theory of polynomials. Oxford University Press, 2002.

[29] F. Shahrokhi e D. W. Matula. "The Maximum Concurrent Flow Problem". Em: Journal of the ACM 37.2 (1990), pp. 318-334. uRL: http://doi.acm.org/10.1145/77600.77620.

[30] N. Weaver. "The Kadison-Singer Problem in discrepancy theory". Em: Discrete Mathematics 278.1-3 (2004), pp. 227-239. URL: http://dx.doi.org/10.1016/S0012-365X (03)00253-X.

[31] F. Zhang. The Schur complement and its applications. Springer Science \& Business Media, 2006. 